\title{
Palladium-Catalyzed Allylic Transposition of (Allyloxy) Iminodiazaphospholidines: A Formal [3,3]-Aza-phospha-oxa-Cope Sigmatropic Rearrangement for the Stereoselective Synthesis of Allylic Amines
}

Ernest E. Lee and Robert A. Batey

\section{Supporting Information}

\section{Experimental Procedures and Characterization Data}

The following general experimental applies for all experiments described in this paper. Benzene and toluene were distilled from sodium-benzophenone ketyl under agron. $\mathrm{CH}_{2} \mathrm{Cl}_{2}$ was distilled from $\mathrm{CaH}_{2}$ under argon. All other solvents were used as obtained prior to use. All commercial reagents, unless otherwise stated, were used as received (Aldrich, Fischer Scientific Ltd. or Lancaster).

IR spectra were obtained on a Perkin-Elmer Spectrum 1000, with samples loaded as films on $\mathrm{NaCl}$ plates. ${ }^{1} \mathrm{H}$ and ${ }^{31} \mathrm{P} \mathrm{NMR}$ spectra were obtained on Varian Gemini 300 spectrometer. ${ }^{13} \mathrm{C}$ NMR spectra were obtained on Unity 400 or 500 spectrometers. Chemical shifts are expressed in ppm values. Proton and carbon chemical shifts in $\mathrm{CDCl}_{3}$ were referenced to $7.26 \mathrm{ppm}$ and $77.23 \mathrm{ppm}$ respectively. Peak multiplicities are designated by the following abbreviations: s, singlet; $d$, doublet; t, triplet; q, quartet; m, multiplet; dd, doublet of doublets; dt, doublet of triplet; b, broad; $J$, coupling constant in Hz. Low resolution mass spectra (MS) were recorded on a Bell and Howell 21-490 spectrometer, high resolution mass spectra (HRMS) were recorded on an AEI MS3074 spectrometer. Melting points were obtained on a Fisher-Johns melting point apparatus and are uncorrected.

Flash column chromatography was carried out on silica gel (60 А, 230 - 400 mesh, obtained from Silicycle Inc.) and was performed with reagent grade solvents. Furthermore, flash column chromatography was performed with either traditional glass columns or on a Biotage SP1 automated flash chromatography system. Analytical thin-layer chromatography (TLC) was performed on precoated aluminum-backed silica gel plates (A1120-100, $60 \AA$ A, F-254 purchased from Silicycle Inc.), visualized with a UV lamp (254 nm), iodine, ninhydrin, potassium permanganate, phosphomolybdic acid (Aldrich), or vanillin. References following the compound names indicate literature articles where the compound has been previously been reported.

Although we have experienced no problems with either tosyl or diphenylphosphoryl azide, appropriate safety measures should be taken. For discussion on the hazards associated with azides, see: Prudent Practices in the Laboratory: Handling and Disposal of Chemicals, National Academy Press: Washington, D.C., 1995. 
Preparation of tosyl iminodiazaphospholidines (11). In a flame dried round bottom flask under $\mathrm{N}_{2}$, allylic alcohol (0.6 mmol) was added to a stirring solution of diazaphospholidinyl dimethylamine 13 $(0.6 \mathrm{mmol})$ in benzene $(6 \mathrm{~mL})$. The reaction was stirred for 6 hours under a dynamic atmosphere of $\mathrm{N}_{2}$ then checked for completion by ${ }^{31} \mathrm{P}$ NMR. Tosyl azide $(0.6 \mathrm{mmol})$ was then slowly added to the stirring solution resulting in rapid effervescence. After azide addition, the reaction was stirred for 15 min and concentrated in vacuo to yield a crude syrup. The crude syrup was then dissolved in a minimum amount of $\mathrm{CH}_{2} \mathrm{Cl}_{2}$ and purified by column chromatography.

\section{(E)-N-(2-Hex-2-enyloxy-1,3-dimethyl-2 $\lambda^{5}$-[1,3,2]diazaphospholidin-2-ylidene)-4-methyl-} benzenesulfonamide (11a).

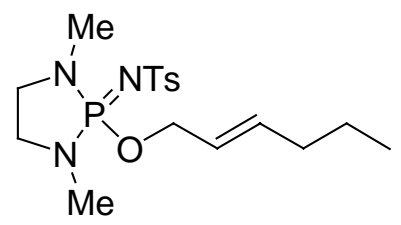

Flash chromatography (EtOAc:hexanes; 1.5:1 + 5\% NEt 3 ) yielded a clear oil $(91 \%)$. $\mathrm{R}_{f}=0.38$ (EtOAc:hexanes; 2:1); IR (Thin Film) v 2930, 1448, 1274, 1160, 1143, 1091, 1041, $997 \mathrm{~cm}^{-1}$; ${ }^{1} \mathrm{H}$ NMR $\left(300 \mathrm{MHz}, \mathrm{CDCl}_{3}\right) \delta 7.83(2 \mathrm{H}, \mathrm{d}, J=8.0 \mathrm{~Hz}), 7.20(2 \mathrm{H}, \mathrm{d}, J=8.0 \mathrm{~Hz}), 5.75(1 \mathrm{H}, \mathrm{dt}, J=15.5,6.5 \mathrm{~Hz})$, $5.52(1 \mathrm{H}, \mathrm{dtt}, J=15.0,6.5,1.0 \mathrm{~Hz}), 4.41(2 \mathrm{H}, \mathrm{ddd}, J=11.0,6.5,1.0 \mathrm{~Hz}), 3.32-3.14(4 \mathrm{H}, \mathrm{m}), 2.50(6 \mathrm{H}$, $\mathrm{d}, J=10.5 \mathrm{~Hz}), 2.38(3 \mathrm{H}, \mathrm{s}), 2.01(2 \mathrm{H}, \mathrm{dt}, J=7.0,7.0 \mathrm{~Hz}), 1.38(2 \mathrm{H}, \mathrm{tq}, J=7.0,7.0 \mathrm{~Hz}), 0.89(3 \mathrm{H}, \mathrm{t}, J$ $=7.0 \mathrm{~Hz}) ;{ }^{13} \mathrm{C} \mathrm{NMR}\left(100 \mathrm{MHz}, \mathrm{CDCl}_{3}\right) \delta 143.2(\mathrm{~d}, J=3.5 \mathrm{~Hz}), 140.8,136.5,128.6,125.9,124.4(\mathrm{~d}, J$ $=5.5 \mathrm{~Hz}), 68.2(\mathrm{~d}, J=7.0 \mathrm{~Hz}), 46.8(\mathrm{~d}, J=13.5 \mathrm{~Hz}), 34.1,31.2(\mathrm{~d}, J=5.0 \mathrm{~Hz}), 21.9,21.3,13.5 ;{ }^{31} \mathrm{P}$ NMR (121 MHz, $\mathrm{CDCl}_{3}$ ) $\delta$ 25.05; MS (EI) m/e (rel intensity) 386 (26), 304 (97), 174 (100), 149 (66), 148 (29), 133 (46), 117 (21), 91 (18); HRMS (EI) m/e calcd $\left(\mathrm{M}^{+}\right)$385.1589, found 385.1598.

\section{(Z)-N-(2-Hex-2-enyloxy-1,3-dimethyl-2 $\lambda^{5}$-[1,3,2]diazaphospholidin-2-ylidene)-4-methyl-} benzenesulfonamide (11b).

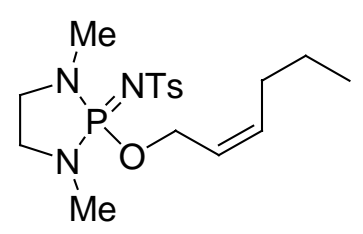

Flash chromatography (EtOAc:hexanes; 1.5:1 + 5\% NEt 3 ) yielded a clear oil $(85 \%)$. $\mathrm{R}_{f}=0.38$ (EtOAc:hexanes; 2:1); IR (Thin Film) v 2939, 2932, 1275, 1162, 1144, 1040, 1007, $949 \mathrm{~cm}^{-1}$; ${ }^{1} \mathrm{H}$ NMR $\left(300 \mathrm{MHz}, \mathrm{CDCl}_{3}\right) \delta 7.83(2 \mathrm{H}, \mathrm{d}, J=8.0 \mathrm{~Hz}), 7.21(2 \mathrm{H}, \mathrm{d}, J=8.0 \mathrm{~Hz}), 5.63(1 \mathrm{H}, \mathrm{dt}, J=11.0,7.5 \mathrm{~Hz})$, $5.51(1 \mathrm{H}, \mathrm{dt}, J=10.5,7.0 \mathrm{~Hz}), 4.52(2 \mathrm{H}, \mathrm{dd}, J=11.0,7.0 \mathrm{~Hz}), 3.31-3.14(4 \mathrm{H}, \mathrm{m}), 2.52(6 \mathrm{H}, \mathrm{d}, J=10.5$ 
Hz), $2.38(3 \mathrm{H}, \mathrm{s}), 2.03(2 \mathrm{H}, \mathrm{td}, J=7.5,7.0 \mathrm{~Hz}), 1.38(2 \mathrm{H}, \mathrm{tq}, J=7.5,7.0 \mathrm{~Hz}), 0.89(3 \mathrm{H}, \mathrm{t}, J=7.5 \mathrm{~Hz})$; ${ }^{13} \mathrm{C}$ NMR $\left(100 \mathrm{MHz}, \mathrm{CDCl}_{3}\right) \delta 142.9(\mathrm{~d}, J=3.5 \mathrm{~Hz}), 140.6,134.9,128.4,125.7,123.8(\mathrm{~d}, J=6.0 \mathrm{~Hz})$, $62.9(\mathrm{~d}, J=7.0 \mathrm{~Hz}), 46.6(\mathrm{~d}, J=13.5 \mathrm{~Hz}), 30.9(\mathrm{~d}, J=5.0 \mathrm{~Hz}), 29.2,22.2,21.0,13.3 ;{ }^{31} \mathrm{P}$ NMR $(121$ $\mathrm{MHz}, \mathrm{CDCl}_{3}$ ) $\delta$ 25.33; MS (EI) m/e (rel intensity) 386 (68), 356 (11), 304 (94), 230 (11), 174 (100), 149 (51), 133 (32), 117 (20); HRMS (EI) m/e calcd $\left(\mathrm{M}^{+}\right)$386.1667, found 386.1673.

\section{(E)- $N$-(1,3-Dimethyl-2-pent-2-enyloxy-2 $\lambda^{5}$-[1,3,2]diazaphospholidin-2-ylidene)-4-methyl-} benzenesulfonamide (11c).

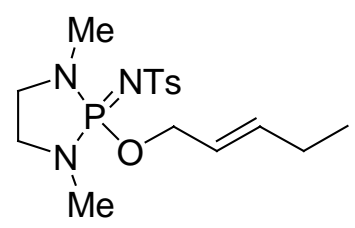

Flash chromatography (EtOAc:hexanes; 2:1 + 5\% $\mathrm{NEt}_{3}$ ) yielded a clear oil $(92 \%) . \mathrm{R}_{f}=0.35$ (EtOAc:hexanes; 3:1); IR (Thin Film) v 2935, 2936, 1580, 1447, 1357, 1271, 1141, 999, $813 \mathrm{~cm}^{-1} ;{ }^{1} \mathrm{H}$ $\operatorname{NMR}\left(300 \mathrm{MHz}, \mathrm{CDCl}_{3}\right) \delta 7.84(2 \mathrm{H}, \mathrm{dd}, J=8.0 \mathrm{~Hz}), 7.20(2 \mathrm{H}, \mathrm{dd}, J=8.0 \mathrm{~Hz}), 5.81(1 \mathrm{H}, \mathrm{dt}, J=15.0$, $6.5 \mathrm{~Hz}), 5.52(1 \mathrm{H}, \mathrm{dtt}, J=15.0,6.5,1.0 \mathrm{~Hz}), 4.40(2 \mathrm{H}, \mathrm{ddd}, J=11.0,6.5,1.0 \mathrm{~Hz}), 3.31-3.12(4 \mathrm{H}, \mathrm{m})$, $2.51(6 \mathrm{H}, \mathrm{d}, J=10.5 \mathrm{~Hz}), 2.38(3 \mathrm{H}, \mathrm{s}), 2.05(2 \mathrm{H}, \mathrm{dq}, J=7.5,6.5 \mathrm{~Hz}), 0.98(3 \mathrm{H}, \mathrm{t}, J=7.5 \mathrm{~Hz}) ;{ }^{13} \mathrm{C}$ NMR $\left(100 \mathrm{MHz} \mathrm{CDCl}_{3}\right) \delta 143.3,141.1,138.5,128.9,126.2,123.5(\mathrm{~d}, J=6.0 \mathrm{~Hz}), 68.6(\mathrm{~d}, J=7.5 \mathrm{~Hz}), 47.0$ $(\mathrm{d}, J=13.5 \mathrm{~Hz}), 31.4(\mathrm{~d}, J=5.5 \mathrm{~Hz}), 25.3,21.5,13.1 ;{ }^{31} \mathrm{P} \mathrm{NMR}\left(121 \mathrm{MHz}, \mathrm{CDCl}_{3}\right) \delta 26.10 ; \mathrm{MS}(\mathrm{EI})$ m/e (rel intensity) 371 (33), 304 (79), 174 (100), 149 (72), 133 (64), 117 (22), 91 (45), 69 (60), 57 (25); HRMS (EI) m/e calcd $\left(\mathrm{M}^{+}\right)$371.1433, found 371.1429.

\section{(Z)-N-(1,3-Dimethyl-2-pent-2-enyloxy-2 $\lambda^{5}$-[1,3,2]diazaphospholidin-2-ylidene)-4-methyl-} benzenesulfonamide (11d).

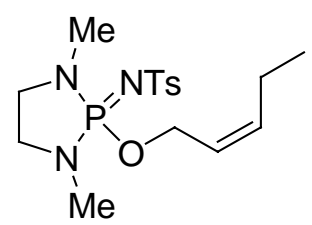

Flash chromatography (EtOAc:hexanes; 2:1 + 5\% $\mathrm{NEt}_{3}$ ) yielded a white solid (93\%). $\mathrm{mp}=84-86{ }^{\circ} \mathrm{C} ; \mathrm{R}_{f}$ $=0.43$ (EtOAc:hexanes; 3:1); IR (Thin Film) v 2934, 2875, 1274, 1163, 1143, 1040, $950 \mathrm{~cm}^{-1}$; ${ }^{1} \mathrm{H}$ NMR $\left(300 \mathrm{MHz}, \mathrm{CDCl}_{3}\right) \delta 7.84(2 \mathrm{H}, \mathrm{d}, J=8.0 \mathrm{~Hz}), 7.21(2 \mathrm{H}, \mathrm{d}, J=8.0 \mathrm{~Hz}), 5.62(1 \mathrm{H}, \mathrm{dt}, J=10.5,7.5 \mathrm{~Hz})$, $5.46(1 \mathrm{H}, \mathrm{dtt}, J=10.5,7.0,1.0 \mathrm{~Hz}), 4.52(2 \mathrm{H}, \mathrm{ddd}, J=10.5,7.5,1.0 \mathrm{~Hz}), 3.31-3.12(4 \mathrm{H}, \mathrm{m}), 2.51(6 \mathrm{H}$, $\mathrm{d}, J=10.5 \mathrm{~Hz}), 2.38(3 \mathrm{H}, \mathrm{s}), 2.08(2 \mathrm{H}, \mathrm{dq}, J=7.5,6.5 \mathrm{~Hz}), 0.97(3 \mathrm{H}, \mathrm{t}, J=7.5 \mathrm{~Hz}) ;{ }^{13} \mathrm{C}$ NMR $(100$ $\left.\mathrm{MHz}, \mathrm{CDCl}_{3}\right) \delta 143.3(\mathrm{~d}, J=4.0 \mathrm{~Hz}), 140.0,137.2,128.8,126.1,123.4(\mathrm{~d}, J=5.5 \mathrm{~Hz}), 63.3(\mathrm{~d}, J=7.5$ 
$\mathrm{Hz}), 47.0(\mathrm{~d}, J=14.0 \mathrm{~Hz}), 31.4(\mathrm{~d}, J=5.5 \mathrm{~Hz}), 25.2,21.4,20.9 ;{ }^{31} \mathrm{P}$ NMR $\left(121 \mathrm{MHz}, \mathrm{CDCl}_{3}\right) \delta 25.28$; MS (EI) m/e (rel intensity) 371 (40), 356 (36), 174 (87), 149 (100), 133 (43), 117 (19), 57 (26); HRMS (EI) $\mathrm{m} / \mathrm{e}$ calcd $\left(\mathrm{M}^{+}\right)$371.1433, found 371.1423 .

\section{(E)-N-(2-[4-(tert-Butyl-dimethyl-silanyloxy)-but-2-enyloxy]-1,3-dimethyl-2 $\lambda^{5}$ -}

\section{[1,3,2]diazaphospholidin-2-ylidene)-4-methyl-benzenesulfonamide (11e).}

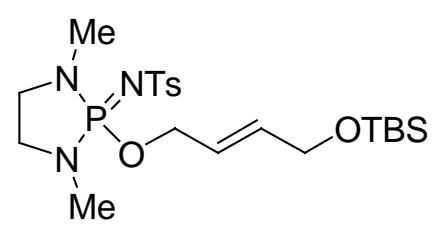

Flash chromatography (EtOAc:hexanes; 1.5:1 + 5\% NEt 3 ) yielded a clear oil $(88 \%)$. $\mathrm{R}_{f}=0.39$ (EtOAc:hexanes; 2:1); IR (Thin Film) v 2930, 2860, 1472, 1273, 1163, 1144, 1020, $840 \mathrm{~cm}^{-1}$; ${ }^{1} \mathrm{H}$ NMR $\left(300 \mathrm{MHz}, \mathrm{CDCl}_{3}\right) \delta 7.78(2 \mathrm{H}, \mathrm{d}, J=8.0 \mathrm{~Hz}), 7.16(2 \mathrm{H}, \mathrm{d}, J=8.0 \mathrm{~Hz}), 5.77(1 \mathrm{H}, \mathrm{dt}, J=15.5,4.5 \mathrm{~Hz})$, $5.74(1 \mathrm{H}, \mathrm{dt}, J=15.5,5.5 \mathrm{~Hz}), 4.40(2 \mathrm{H}, \mathrm{dd}, J=11.0,5.0 \mathrm{~Hz}), 4.10(2 \mathrm{H}, \mathrm{dd}, J=4.0,1.0 \mathrm{~Hz}), 3.24-3.06$ $(4 \mathrm{H}, \mathrm{m}), 2.44(6 \mathrm{H}, \mathrm{d}, J=10.5 \mathrm{~Hz}), 2.33(3 \mathrm{H}, \mathrm{s}), 0.85(9 \mathrm{H}, \mathrm{s}), 0.01(6 \mathrm{H}, \mathrm{s}) ;{ }^{13} \mathrm{C} \mathrm{NMR}\left(100 \mathrm{MHz}, \mathrm{CDCl}_{3}\right)$ $\delta 143.30(\mathrm{~d}, J=3.5 \mathrm{~Hz}), 141.00,134.40,128.80,126.10,123.80(\mathrm{~d}, J=6.0 \mathrm{~Hz}), 67.70(\mathrm{~d} \mathrm{~Hz}), 62.60$, $47.00(\mathrm{~d}, J=13.5 \mathrm{~Hz}), 31.40(\mathrm{~d}, J=4.5 \mathrm{~Hz}), 25.90,21.40,18.40,-5.30 ;{ }^{31} \mathrm{P}$ NMR $\left(121 \mathrm{MHz}, \mathrm{CDCl}_{3}\right)$ $\delta$ 24.94; MS (EI) m/e (rel intensity) 488 (12), 430 (51), 418 (31), 360 (74), 304 (23), 207 (34), 174 (100), 149 (30), 133 (25); HRMS (EI) m/e calcd ( $\mathrm{M}^{+}$) 488.2168, found 488.2160.

\section{(Z)-N-(2-[4-(tert-Butyl-dimethyl-silanyloxy)-but-2-enyloxy]-1,3-dimethyl-2 $\lambda^{5}$ -}

\section{$[1,3,2]$ diazaphospholidin-2-ylidene)-4-methyl-benzenesulfonamide (11f).}

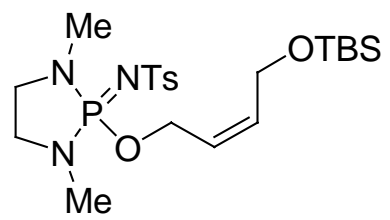

Flash chromatography (EtOAc:hexanes; 1.5:1 + 5\% NEt 3 ) yielded a clear oil $(91 \%) . \mathrm{R}_{f}=0.41$ (EtOAc:hexanes; 2:1); IR (Thin Film) v 2929, 1472, 1384, 1274, 1144, 1003, $840 \mathrm{~cm}^{-1}$; ${ }^{1} \mathrm{H}$ NMR (300 $\left.\mathrm{MHz} \mathrm{CDCl}_{3}\right) \delta 7.82(2 \mathrm{H}, \mathrm{d}, J=8.0 \mathrm{~Hz}), 7.21(2 \mathrm{H}, \mathrm{d}, J=8.0 \mathrm{~Hz}), 5.70(1 \mathrm{H}, \mathrm{dt}, J=11.0,6.0 \mathrm{~Hz}), 5.69$ $(1 \mathrm{H}, \mathrm{dtt}, J=11.0,6.5,1.5 \mathrm{~Hz}), 4.56(2 \mathrm{H}, \mathrm{ddd}, J=10.5,7.5,1.0 \mathrm{~Hz}), 4.22(2 \mathrm{H}, \mathrm{d}, J=6.0 \mathrm{~Hz}), 3.22-3.14$ $(4 \mathrm{H}, \mathrm{m}), 2.51(6 \mathrm{H}, \mathrm{d}, J=10.5 \mathrm{~Hz}), 2.38(3 \mathrm{H}, \mathrm{s}), 0.89(9 \mathrm{H}, \mathrm{s}), 0.06(6 \mathrm{H}, \mathrm{s}) ;{ }^{13} \mathrm{C} \mathrm{NMR}\left(100 \mathrm{MHz}, \mathrm{CDCl}_{3}\right)$ $\delta 143.30(\mathrm{~d}, J=4.0 \mathrm{~Hz}), 141.20,133.70(\mathrm{~d}, J=0.5 \mathrm{~Hz}), 129.00,126.20,125.10(\mathrm{~d}, J=6.0 \mathrm{~Hz}), 63.70$ $(\mathrm{d}, J=7.5 \mathrm{~Hz}), 59.80,47.10(\mathrm{~d}, J=13.5 \mathrm{~Hz}), 31.60(\mathrm{~d}, J=5.0 \mathrm{~Hz}), 26.00,21.60,18.40,-5.10 ;{ }^{31} \mathrm{P}$ NMR 
(121 MHz, $\left.\mathrm{CDCl}_{3}\right) \delta$ 25.41; MS (EI) m/e (rel intensity) 488 (17), 430 (41), 360 (78), 304 (56), 207 (207), 174 (100), 149 (22); HRMS (EI) m/e calcd $\left(\mathrm{M}^{+}\right)$488.2168, found 488.2165.

\section{(E)-N-(2-But-2-enyloxy-1,3-dimethyl-2 $\lambda^{5}$-[1,3,2] diazaphospholidin-2-ylidene)-4-methyl-} benzenesulfonamide (11g).

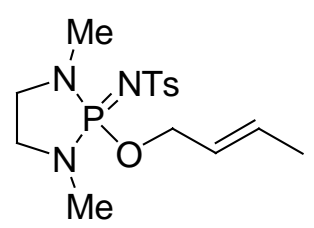

Flash Chromatography (EtOAc:hexanes; $1.5: 1+5 \% \mathrm{NEt})$ yielded a white solid $(84 \%) . \mathrm{mp}=42-44{ }^{\circ} \mathrm{C}$; $\mathrm{R}_{f}=0.40$ (EtOAc:hexanes; 2:1); IR (Thin Film) v 2939, 1273, 1163, 1143, 1040, 1003, $968 \mathrm{~cm}^{-1} ;{ }^{1} \mathrm{H}$ NMR (300 MHz, $\left.\mathrm{CDCl}_{3}\right) \delta 7.83(2 \mathrm{H}, \mathrm{d}, J=8.0 \mathrm{~Hz}), 7.20(2 \mathrm{H}, \mathrm{d}, J=8.0 \mathrm{~Hz}), 5.77(1 \mathrm{H}, \mathrm{dq}, J=15.0,6.5$ Hz), $5.55(1 \mathrm{H}, \mathrm{dtq}, J=15.0,6.0,1.0 \mathrm{~Hz}), 4.39(2 \mathrm{H}, \mathrm{ddd}, J=10.5,6.5,0.5 \mathrm{~Hz}), 3.28-3.12(4 \mathrm{H}, \mathrm{m}), 2.50$ $(6 \mathrm{H}, \mathrm{d}, J=10.5 \mathrm{~Hz}), 2.38(3 \mathrm{H}, \mathrm{s}), 1.70(3 \mathrm{H}, \mathrm{dd}, J=6.5,1.0 \mathrm{~Hz}) ;{ }^{13} \mathrm{C} \mathrm{NMR}\left(100 \mathrm{MHz}, \mathrm{CDCl}_{3}\right) \delta 143.4$ $(\mathrm{d}, J=4.0 \mathrm{~Hz}), 141.1,131.9,128.9,126.2,125.9(\mathrm{~d}, J=6.0 \mathrm{~Hz}), 68.7$ (d, $J=7.5 \mathrm{~Hz}), 47.1(\mathrm{~d}, J=13.0$ $\mathrm{Hz}), 31.5(\mathrm{~d}, J=4.5 \mathrm{~Hz}), 21.5,17.8 ;{ }^{31} \mathrm{P} \mathrm{NMR}\left(121 \mathrm{MHz}, \mathrm{CDCl}_{3}\right) \delta 25.00 ; \mathrm{MS}$ (EI) $m / e$ (rel intensity) 357 (2), 302 (22), 202 (17), 174 (100), 149 (97), 133 (56), 117 (30); HRMS (EI) m/e calcd (M+) 357.1276 , found 357.1278 .

\section{(Z)- $N$-(2-But-2-enyloxy-1,3-dimethyl-2 $\lambda^{5}$-[1,3,2] diazaphospholidin-2-ylidene)-4-methyl-} benzenesulfonamide (11h).

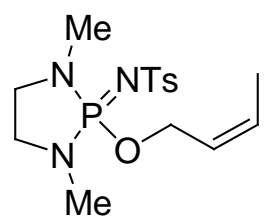

Flash chromatography (EtOAc:hexanes; 1.5:1 + 5\% $\mathrm{NEt}_{3}$ ) yielded a clear oil (86\%). $\mathrm{R}_{f}=0.40$ (EtOAc:hexanes; 2:1); ${ }^{1} \mathrm{H}$ NMR $\left(300 \mathrm{MHz}, \mathrm{CDCl}_{3}\right) \delta 7.82(2 \mathrm{H}, \mathrm{d}, J=8.0 \mathrm{~Hz}), 7.20(2 \mathrm{H}, \mathrm{d}, J=8.0 \mathrm{~Hz})$, $5.72(1 \mathrm{H}, \mathrm{dqt}, J=10.5,7.0,0.5 \mathrm{~Hz}), 5.52(1 \mathrm{H}, \mathrm{dtq}, J=10.5,7.0,1.0 \mathrm{~Hz}), 4.52(2 \mathrm{H}, \mathrm{dd}, J=11.0,7.0$ $\mathrm{Hz}), 3.29-3.12(4 \mathrm{H}, \mathrm{m}), 2.49(6 \mathrm{H}, \mathrm{d}, J=10.5 \mathrm{~Hz}), 2.37(3 \mathrm{H}, \mathrm{s}), 1.68(3 \mathrm{H}, \mathrm{d}, J=7.0 \mathrm{~Hz}){ }^{13} \mathrm{C} \mathrm{NMR}(100$ $\left.\mathrm{MHz}, \mathrm{CDCl}_{3}\right) \delta 142.9(\mathrm{~d}, J=3.0 \mathrm{~Hz}), 140.6,129.5,128.4(\mathrm{~d}, J=3.5 \mathrm{~Hz}), 125.7,124.4(\mathrm{~d}, J=5.5 \mathrm{~Hz})$, $62.5(\mathrm{~d}, J=7.0 \mathrm{~Hz}), 46.6(\mathrm{~d}, J=13.5 \mathrm{~Hz}), 30.9(\mathrm{~d}, J=5.0 \mathrm{~Hz}), 21.1,12.9 ;{ }^{31} \mathrm{P} \mathrm{NMR}\left(121 \mathrm{MHz}, \mathrm{CDCl}_{3}\right)$ $\delta$ 25.12; MS (EI) m/e (rel intensity) 358 (68), 304 (62), 174 (100), 149 (54), 133 (33), 117 (25); HRMS (EI) $\mathrm{m} / \mathrm{e}$ calcd $\left(\mathrm{M}^{+}\right) 358.1354$, found 358.1351. 


\section{$N$-[1,3-Dimethyl-2-(2-methyl-allyloxy)-2 $\lambda^{5}$-[1,3,2] diazaphospholidin-2-ylidene]-4-methyl-}

benzenesulfonamide (11i).

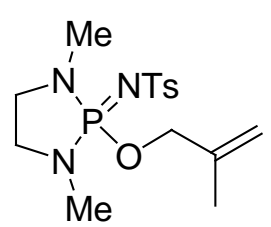

Flash chromatography (EtOAc:hexanes; 2:1 + 5\% $\mathrm{NEt}_{3}$ ) yielded a clear oil (95\%). $\mathrm{R}_{f}=0.38$ (EtOAc:hexanes; 3:1); IR (Thin Film) v 2939, 1448, 1273, 1162, 1143, 1040, 1008, $950 \mathrm{~cm}^{-1}$; ${ }^{1} \mathrm{H}$ NMR $\left(300 \mathrm{MHz}, \mathrm{CDCl}_{3}\right) \delta 7.84(2 \mathrm{H}, \mathrm{d}, J=8.0 \mathrm{~Hz}), 7.21(2 \mathrm{H}, \mathrm{d}, J=8.0 \mathrm{~Hz}), 4.99(1 \mathrm{H}, \mathrm{s}), 4.92(1 \mathrm{H}, \mathrm{s}), 4.34$ $(2 \mathrm{H}, \mathrm{d}, J=10.0 \mathrm{~Hz}), 3.29-3.16(4 \mathrm{H}, \mathrm{m}), 2.52(6 \mathrm{H}, \mathrm{d}, J=10.5 \mathrm{~Hz}), 2.38(3 \mathrm{H}, \mathrm{s}), 1.74(3 \mathrm{H}, \mathrm{s}) ;{ }^{13} \mathrm{C} \mathrm{NMR}$ $\left(100 \mathrm{MHz}, \mathrm{CDCl}_{3}\right) \delta 143.3(\mathrm{~d}, J=3.0 \mathrm{~Hz}), 141.1,140.2(\mathrm{~d}, J=6.0 \mathrm{~Hz}), 128.9,126.1,113.6,71.1(\mathrm{~d}, J$ $=7.5 \mathrm{~Hz}), 47.1(\mathrm{~d}, J=13.0 \mathrm{~Hz}), 31.5(\mathrm{~d}, J=4.5 \mathrm{~Hz}), 21.5,19.2 ;{ }^{31} \mathrm{P} \mathrm{NMR}\left(121 \mathrm{MHz}, \mathrm{CDCl}_{3}\right) \delta 25.05$; MS (EI) m/e (rel intensity) 357 (5), 302 (20), 202 (42), 149 (100), 133 (83), 117 (22), 85 (22); HRMS (EI) $m / e$ calcd $\left(\mathrm{M}^{+}\right)$357.1276, found 357.1264.

\section{$N$-[1,3-Dimethyl-2-(1-methyl-allyloxy)-2 $\lambda^{5}$-[1,3,2] diazaphospholidin-2-ylidene]-4-methyl-} benzenesulfonamide (11j).

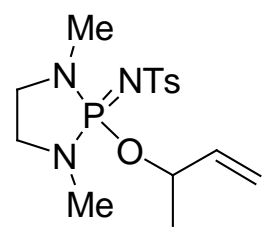

Flash chromatography (EtOAc:hexanes; 2:1 + 5\% $\mathrm{NEt}_{3}$ ) yielded a clear oil $(91 \%)$. $\mathrm{R}_{f}=0.38$ (EtOAc:hexanes; 3:1); IR (Thin Film) v 2979, 2932, 1357, 1272, 1163, 1143, 1006, $949 \mathrm{~cm}^{-1}$; ${ }^{1} \mathrm{H}$ NMR $\left(300 \mathrm{MHz}, \mathrm{CDCl}_{3}\right) \delta 7.83(2 \mathrm{H}, \mathrm{d}, J=8.0 \mathrm{~Hz}), 7.21(2 \mathrm{H}, \mathrm{d}, J=8.0 \mathrm{~Hz}), 5.82(1 \mathrm{H}, \mathrm{ddd}, J=17.0,10.5$, $6.5 \mathrm{~Hz}), 5.24(1 \mathrm{H}, \mathrm{d}, J=17.5 \mathrm{~Hz}), 5.13(1 \mathrm{H}, \mathrm{d}, J=10.5 \mathrm{~Hz}), 4.84(1 \mathrm{H}, \mathrm{dq}, J=16.0,6.5 \mathrm{~Hz}), 3.24-3.14$ $(4 \mathrm{H}, \mathrm{m}), 2.48(3 \mathrm{H}, \mathrm{d}, J=10.5 \mathrm{~Hz}), 2.44(3 \mathrm{H}, \mathrm{d}, J=10.5 \mathrm{~Hz}), 2.38(3 \mathrm{H}, \mathrm{s}), 1.33(3 \mathrm{H}, \mathrm{d}, J=6.5 \mathrm{~Hz}) ;{ }^{13} \mathrm{C}$ $\operatorname{NMR}\left(100 \mathrm{MHz}, \mathrm{CDCl}_{3}\right) \delta 143.3,141.0,138.3(\mathrm{~d}, J=4.5 \mathrm{~Hz}), 128.8,126.1,116.2,75.9(\mathrm{~d}, J=7.5 \mathrm{~Hz})$, $46.9(2 \mathrm{~d}, J=13.0,13.0 \mathrm{~Hz}), 31.2(2 \mathrm{~d}, J=4.5,4.5 \mathrm{~Hz}), 22.2(\mathrm{~d}, J=6.0 \mathrm{~Hz}), 21.4 ;{ }^{31} \mathrm{P} \mathrm{NMR}(121 \mathrm{MHz}$, $\left.\mathrm{CDCl}_{3}\right) \delta 23.83$; MS (EI) m/e (rel intensity) 357 (3), 302 (21), 188 (98), 149 (100), 133 (47), 57 (29); HRMS (EI) m/e calcd $\left(\mathrm{M}^{+}\right)$357.1276, found 357.1266.

\section{$N$-[2-(Cyclohex-2-enyloxy)-1,3-dimethyl-2 $\lambda^{5}$-[1,3,2] diazaphospholidin-2-ylidene]-4-methyl- benzenesulfonamide (11k).}




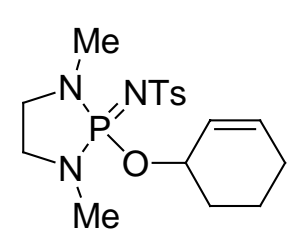

Flash chromatography (EtOAc:hexanes; 2:1 $+5 \% \mathrm{NEt}_{3}$ ) yielded a clear oil $(89 \%) . \mathrm{R}_{f}=0.40$ (EtOAc:hexanes; 3:1); IR (Thin Film) v 2936, 1272, 1150, 1142, 986, $949 \mathrm{~cm}^{-1} ;{ }^{1} \mathrm{H}$ NMR (300 MHz, $\left.\mathrm{CDCl}_{3}\right) \delta 7.83(2 \mathrm{H}, \mathrm{d}, J=8.0 \mathrm{~Hz}), 7.21(2 \mathrm{H}, \mathrm{d}, J=8.0 \mathrm{~Hz}), 5.93(1 \mathrm{H}, \mathrm{dt}, J=10.0,3.5 \mathrm{~Hz}), 5.64(1 \mathrm{H}$, ddt, $J=10.0,4.0,2.0 \mathrm{~Hz}), 4.88-4.78(1 \mathrm{H}, \mathrm{bm}), 3.30-3.12(4 \mathrm{H}, \mathrm{m}), 3.22(3 \mathrm{H}, \mathrm{d}, J=10.5 \mathrm{~Hz}), 3.21(3 \mathrm{H}$, d, $J=10.5 \mathrm{~Hz}), 2.38(3 \mathrm{H}, \mathrm{s}), 2.12-1.52(6 \mathrm{H}, \mathrm{m}) ;{ }^{13} \mathrm{C} \mathrm{NMR}\left(100 \mathrm{MHz}, \mathrm{CDCl}_{3}\right) \delta 143.4(\mathrm{~d}, J=3.0 \mathrm{~Hz})$, 141.0, 133.3, 128.9, 126.2, 126.1 (d, $J=4.5 \mathrm{~Hz}), 72.7(\mathrm{~d}, J=8.5 \mathrm{~Hz}), 47.0(2 \mathrm{~d}, J=13.0,13.0 \mathrm{~Hz}), 31.4$ $(2 \mathrm{~d}, J=7.0,7.0 \mathrm{~Hz}), 30.1(\mathrm{~d}, J=5.0 \mathrm{~Hz}), 24.8,21.5,18.6 ;{ }^{31} \mathrm{P} \mathrm{NMR}\left(121 \mathrm{MHz}, \mathrm{CDCl}_{3}\right) \delta 23.91 ; \mathrm{MS}$ (EI) m/e (rel intensity) 383 (6), 319 (36), 304 (48), 228 (78), 149 (51), 133 (100), 79 (44), 57 (55); HRMS (EI) $m / e$ calcd $\left(\mathrm{M}^{+}\right) 383.1433$, found 383.1433 .

\section{(E)- $N$-[2-(1-Ethyl-but-2-enyloxy)-1,3-dimethyl-2 $\lambda^{5}$-[1,3,2] diazaphospholidin-2-ylidene]-4-methyl-} benzenesulfonamide (111).

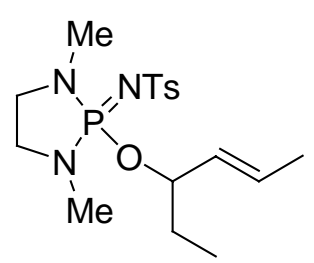

Flash chromatography (EtOAc:hexanes; 2:1 $+5 \% \mathrm{NEt}_{3}$ ) yielded a clear oil $(93 \%) . \mathrm{R}_{f}=0.36$ (EtOAc:hexanes; 3:1); IR (Thin Film) v 2934, 1447, 1340, 1272, 1164, 1041, 989, 950, $670 \mathrm{~cm}^{-1}$; ${ }^{1} \mathrm{H}$ $\operatorname{NMR}\left(300 \mathrm{MHz}, \mathrm{CDCl}_{3}\right) \delta 7.83(2 \mathrm{H}, \mathrm{d}, J=8.0 \mathrm{~Hz}), 7.20(2 \mathrm{H}, \mathrm{d}, J=8.0 \mathrm{~Hz}), 5.70(1 \mathrm{H}, \mathrm{dq}, J=15.0$, $6.5 \mathrm{~Hz}), 5.38(1 \mathrm{H}, \mathrm{ddq}, J=15.5,8.0,2.0 \mathrm{~Hz}), 4.56(1 \mathrm{H}, \mathrm{ddt}, J=8.5,8.5,7.0 \mathrm{~Hz}), 3.28-3.18(4 \mathrm{H}, \mathrm{m})$, $2.47(3 \mathrm{H}, \mathrm{d}, J=10.5 \mathrm{~Hz}), 2.43(3 \mathrm{H}, \mathrm{d}, J=10.0 \mathrm{~Hz}), 2.37(3 \mathrm{H}, \mathrm{s}), 1.67$ (3H, dd, $J=6.5,2.0 \mathrm{~Hz}), 1.68$ $1.45(2 \mathrm{H}, \mathrm{m}), 0.87(3 \mathrm{H}, \mathrm{t}, J=7.5 \mathrm{~Hz}) ;{ }^{13} \mathrm{C} \mathrm{NMR}\left(100 \mathrm{MHz}, \mathrm{CDCl}_{3}\right) \delta 143.4,140.8,130.4(\mathrm{~d}, J=3.0$ $\mathrm{Hz}), 129.9,128.6,126.1,81.5(\mathrm{~d}, J=12.5 \mathrm{~Hz}), 46.9(2 \mathrm{~d}, J=14.0,14.0 \mathrm{~Hz}), 31.5(2 \mathrm{~d}, J=4.5,4.5 \mathrm{~Hz})$, $29.1(\mathrm{~d}, J=7.5 \mathrm{~Hz}), 21.4,17.6,9.6 ;{ }^{31} \mathrm{P}$ NMR $\left(121 \mathrm{MHz}, \mathrm{CDCl}_{3}\right) \delta 23.64$; MS (EI) $m / e$ (rel intensity) 385 (5), 230 (75), 202 (29), 151 (19), 149 (30), 148 (40), 133 (100), 91 (17), 57 (35); HRMS (EI) m/e calcd $\left(\mathrm{M}^{+}\right)$385.1589, found 385.1589.

(E)- $N$-[1,3-Dimethyl-2-(3-phenyl-allyloxy)-2 $\lambda^{5}$-[1,3,2] diazaphospholidin-2-ylidene]-4-methylbenzenesulfonamide $(11 \mathrm{~m})$. 


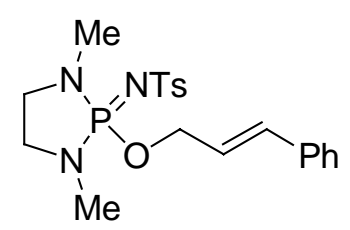

Flash chromatography (EtOAc:hexanes; 1:1 + 5\% $\mathrm{NEt}_{3}$ ) yielded a white solid $(94 \%) . \mathrm{mp}=126-127{ }^{\circ} \mathrm{C}$; $\mathrm{R}_{f}=0.33$ (EtOAc:hexanes; 2:1); IR (Thin Film) v 2939, 1495, 1448, 1357, 1272, 1162, 1142, 1040, 997 $\mathrm{cm}^{-1} ;{ }^{1} \mathrm{H}$ NMR $\left(300 \mathrm{MHz}, \mathrm{CDCl}_{3}\right) \delta 7.85(2 \mathrm{H}, \mathrm{d}, J=8.0 \mathrm{~Hz}), 7.36-7.26(5 \mathrm{H}, \mathrm{m}), 7.20(2 \mathrm{H}, \mathrm{d}, J=8.0$ $\mathrm{Hz}), 6.63(1 \mathrm{H}, \mathrm{d}, J=16.0 \mathrm{~Hz}), 6.22(1 \mathrm{H}, \mathrm{dt}, J=16.0,6.5 \mathrm{~Hz}), 4.62(2 \mathrm{H}, \mathrm{ddd}, J=11.0,6.5,1.5 \mathrm{~Hz})$, 3.30-3.15 (4H, m), $2.52(6 \mathrm{H}, \mathrm{d}, J=10.5 \mathrm{~Hz}), 2.37(3 \mathrm{H}, \mathrm{s}) ;{ }^{13} \mathrm{C} \mathrm{NMR}\left(100 \mathrm{MHz}, \mathrm{CDCl}_{3}\right) \delta 143.3(\mathrm{~d}, J=$ $4.0 \mathrm{~Hz}), 141.2,136.0,134.5,130.0,128.8,128.5,126.8,126.2,123.7$ (d, $J=5.5 \mathrm{~Hz}), 68.6$ (d, $J=8.0$ $\mathrm{Hz}), 47.1(\mathrm{~d}, J=5.5 \mathrm{~Hz}), 31.6(\mathrm{~d}, J=4.5 \mathrm{~Hz}), 21.5 ;{ }^{31} \mathrm{P} \mathrm{NMR}\left(121 \mathrm{MHz}, \mathrm{CDCl}_{3}\right) \delta 26.14 ; \mathrm{MS}(\mathrm{EI}) \mathrm{m} / e$ (rel intensity) 419 (5), 264 (65), 174 (36), 149 (34), 133 (99), 117 (100), 91 (82), 65 (29); HRMS (EI) $m / e$ calcd $\left(\mathrm{M}^{+}\right)$419.1424, found 419.1433.

\section{(E)-N-[1,3-Dimethyl-2-(1-methyl-3-phenyl-allyloxy)-2 $\lambda^{5}$-[1,3,2] diazaphospholidin-2-ylidene]-4-} methyl-benzenesulfonamide (11n).

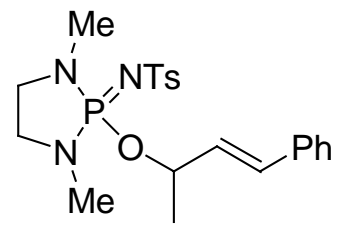

Flash chromatography (EtOAc:hexanes; 2:1 + 5\% $\mathrm{NEt}_{3}$ ) yielded a clear oil $(91 \%)$. $\mathrm{R}_{f}=0.36$ (EtOAc:hexanes; 3:1); IR (Thin Film) v 2933, 1494, 1448, 1271, 1165, 1041, 973, $669 \mathrm{~cm}^{-1}$; ${ }^{1} \mathrm{H}$ NMR $\left(300 \mathrm{MHz}, \mathrm{CDCl}_{3}\right) \delta 7.84(2 \mathrm{H}, \mathrm{d}, J=8.0 \mathrm{~Hz}), 7.36-7.23(5 \mathrm{H}, \mathrm{m}), 7.19(2 \mathrm{H}, \mathrm{d}, J=8.5 \mathrm{~Hz}), 6.56(1 \mathrm{H}, \mathrm{d}$, $J=16.0 \mathrm{~Hz}), 6.15(1 \mathrm{H}, \mathrm{dd}, J=16.0,7.5 \mathrm{~Hz}), 5.04(1 \mathrm{H}, \mathrm{ddq}, J=8.0,7.5,6.5 \mathrm{~Hz}), 3.3-3.12(4 \mathrm{H}, \mathrm{m}), 2.46$ $(6 \mathrm{H}, \mathrm{dd}, J=10.5,1.0 \mathrm{~Hz}), 2.36(3 \mathrm{H}, \mathrm{s}), 1.43(3 \mathrm{H}, \mathrm{dd}, J=6.5,1.0 \mathrm{~Hz}) ;{ }^{13} \mathrm{C} \mathrm{NMR}\left(100 \mathrm{MHz}, \mathrm{CDCl}_{3}\right)$ $\delta 143.4(\mathrm{~d}, J=3.0 \mathrm{~Hz}), 141.0,136.0,132.1,129.3(\mathrm{~d}, J=3.0 \mathrm{~Hz}), 128.9,128.8,128.3,126.7,126.2$, $76.3(\mathrm{~d}, J=7.5 \mathrm{~Hz}), 47.0(2 \mathrm{~d}, J=13.5,14.5 \mathrm{~Hz}), 31.5(2 \mathrm{~d}, J=4.5,4.5 \mathrm{~Hz}), 22.6$ (d, $J=7.0 \mathrm{~Hz}), 21.4$; ${ }^{31} \mathrm{P}$ NMR (121 MHz, $\left.\mathrm{CDCl}_{3}\right) \delta 23.87$; MS (EI) m/e (rel intensity) 433 (1), 303 (9), 279 (100), 279 (18), 239 (42), 133 (18), 91 (21); HRMS (EI) m/e calcd ( $\mathrm{M}^{+}$) 433.1589, found 433.1581.

\section{$N$-[1,3-Dimethyl-2-(3-methyl-but-2-enyloxy)-2 $\lambda^{5}$-[1,3,2]diazaphospholidin-2-ylidene]-4-methyl- benzenesulfonamide (11p).}




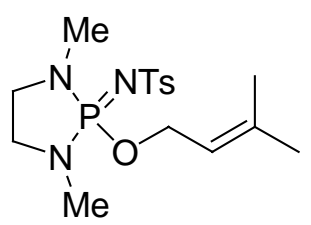

Flash chromatography (EtOAc:hexanes; 2:1 $+5 \% \mathrm{NEt}_{3}$ ) yielded a clear oil $(92 \%) . \mathrm{R}_{f}=0.35$ (EtOAc:hexanes; 3:1); IR (Thin Film) v 2934, 1273, 1162, 1143, 1040, 995, $674 \mathrm{~cm}^{-1} ;{ }^{1} \mathrm{H}$ NMR (300 $\left.\mathrm{MHz}, \mathrm{CDCl}_{3}\right) \delta 7.83(2 \mathrm{H}, \mathrm{d}, J=8.0 \mathrm{~Hz}), 7.21(2 \mathrm{H}, \mathrm{d}, J=8.5 \mathrm{~Hz}), 5.32(1 \mathrm{H}, \mathrm{td}, J=7.5,1.5 \mathrm{~Hz}), 4.46$ $(2 \mathrm{H}, \mathrm{dd}, J=11.0,7.5 \mathrm{~Hz}), 3.28-3.12(4 \mathrm{H}, \mathrm{m}), 2.49(6 \mathrm{H}, \mathrm{d}, J=11.0 \mathrm{~Hz}), 2.38(3 \mathrm{H}, \mathrm{s}), 1.74(3 \mathrm{H}, \mathrm{s}), 1.68$ $(3 \mathrm{H}, \mathrm{s}) ;{ }^{13} \mathrm{C}$ NMR $\left(100 \mathrm{MHz}, \mathrm{CDCl}_{3}\right) \delta 143.4(\mathrm{~d}, J=4.0 \mathrm{~Hz}), 141.0,139.8,128.9,126.2,119.4(\mathrm{~d}, J=$ $4.5 \mathrm{~Hz}), 64.7(\mathrm{~d}, J=7.5 \mathrm{~Hz}), 47.1(\mathrm{~d}, J=13.5 \mathrm{~Hz}), 31.5(\mathrm{~d}, J=4.5 \mathrm{~Hz}), 25.9,21.5,18.2 ;{ }^{31} \mathrm{P}$ NMR $(121$ $\mathrm{MHz}, \mathrm{CDCl}_{3}$ ) $\delta$ 24.94; MS (EI) m/e (rel intensity) 371 (25), 303 (76), 239 (48), 216 (99), 174 (80), 148 (99), 133 (91), 91 (100); HRMS (EI) m/e calcd $\left(\mathrm{M}^{+}\right)$371.1431, found 371.1433.

\section{$(E)-(S)-N$-[1,3-Dimethyl-2-(1-methyl-pent-2-enyloxy)-2 $\lambda^{5}$-[1,3,2] diazaphospholidin-2-ylidene]-4-} methyl-benzenesulfonamide (11q).

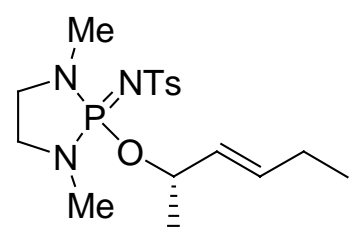

Flash chromatography (EtOAc:hexanes; 2:1 $\left.+5 \% \mathrm{NEt}_{3}\right)$ yielded a clear oil $(86 \%) .[\alpha]_{\mathrm{D}}=-9.8^{\mathrm{o}}\left(\mathrm{CHCl}_{3}\right.$, c=1.0); $\mathrm{R}_{f}=0.36$ (EtOAc:hexanes; 2:1); IR (Thin Film) v 2932, 1447, 1357, 1271, 1163, 1043, 990, 672 $\mathrm{cm}^{-1} ;{ }^{1} \mathrm{H}$ NMR $\left(300 \mathrm{MHz}, \mathrm{CDCl}_{3}\right) \delta 7.83(2 \mathrm{H}, \mathrm{d}, J=8.0 \mathrm{~Hz}), 7.20(2 \mathrm{H}, \mathrm{d}, J=8.5 \mathrm{~Hz}), 5.72(1 \mathrm{H}, \mathrm{dt}, J=$ $15.5,6.0 \mathrm{~Hz}), 5.42(1 \mathrm{H}, \mathrm{ddt}, J=15.5,7.5,1.5 \mathrm{~Hz}), 4.88-4.76(1 \mathrm{H}, \mathrm{m}), 3.30-3.78(4 \mathrm{H}, \mathrm{m}), 2.48(3 \mathrm{H}, \mathrm{d}, J$ $=10.5 \mathrm{~Hz}), 2.44(3 \mathrm{H}, \mathrm{d}, J=10.0 \mathrm{~Hz}), 2.37(3 \mathrm{H}, \mathrm{s}), 2.01(2 \mathrm{H}, \mathrm{dq}, J=7.5,6.5 \mathrm{~Hz}), 1.32(3 \mathrm{H}, \mathrm{d}, J=6.5$ $\mathrm{Hz}), 0.96(3 \mathrm{H}, \mathrm{t}, J=7.5 \mathrm{~Hz}) ;{ }^{13} \mathrm{C} \mathrm{NMR}\left(100 \mathrm{MHz}, \mathrm{CDCl}_{3}\right) \delta 143.5(\mathrm{~d}, J=3.5 \mathrm{~Hz}), 141.0,135.5,129.4$ $(\mathrm{d}, J=3.5 \mathrm{~Hz}), 128.9,126.2,76.5(\mathrm{~d}, J=8.0 \mathrm{~Hz}), 47.0(2 \mathrm{~d}, J=13.5 \mathrm{~Hz}), 31.5(2 \mathrm{~d}, J=15.0 \mathrm{~Hz}), 25.2$, $22.7(\mathrm{~d}, J=6.5 \mathrm{~Hz}), 21.5,13.2 ;{ }^{31} \mathrm{P}$ NMR $\left(121 \mathrm{MHz}, \mathrm{CDCl}_{3}\right) \delta 23.69$; MS (EI) $m / e$ (rel intensity) 385 (5), 356 (13), 304 (39), 292 (16), 239 (46), 230 (86), 188 (30), 148 (62), 133 (100), 91 (47); HRMS (EI) $m / e$ calcd $\left(\mathrm{M}^{+}\right) 385.1589$, found 385.1593 .

(Z)-(S)- $N$-[1,3-Dimethyl-2-(1-methyl-pent-2-enyloxy)-2 $\lambda^{5}$-[1,3,2]diazaphospholidin-2-ylidene]-4methyl-benzenesulfonamide (11r). 


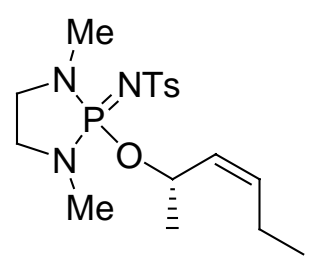

Flash chromatography (EtOAc:hexanes; $\left.2: 1+5 \% \mathrm{NEt}_{3}\right)$ yielded a clear oil $(87 \%)$. $[\alpha]_{\mathrm{D}}=+18.5^{\mathrm{o}}$ $\left(\mathrm{CHCl}_{3}, \mathrm{c}=1.2\right) ; \mathrm{R}_{f}=0.34$ (EtOAc:hexanes; 2:1); IR (Thin Film) v 2932, 1447, 1357, 1271, 1163, 1043, 990, $672 \mathrm{~cm}^{-1}$; ${ }^{1} \mathrm{H}$ NMR (300 MHz, $\left.\mathrm{CDCl}_{3}\right) \delta 7.82(2 \mathrm{H}, \mathrm{d}, J=8.0 \mathrm{~Hz}), 7.20(2 \mathrm{H}, \mathrm{d}, J=8.0 \mathrm{~Hz}), 5.45$ $(1 \mathrm{H}, \mathrm{dt}, J=11.0,7.0 \mathrm{~Hz}), 5.36(1 \mathrm{H}, \mathrm{dd}, J=10.5,9.0 \mathrm{~Hz}), 5.26-5.15(1 \mathrm{H}, \mathrm{m}), 3.28-3.10(4 \mathrm{H}, \mathrm{m}), 2.46$ $(6 \mathrm{H}, \mathrm{dd}, J=10.5,0.5 \mathrm{~Hz}), 2.37(3 \mathrm{H}, \mathrm{s}), 2.12-2.00(2 \mathrm{H}, \mathrm{m}), 1.30(3 \mathrm{H}, \mathrm{dd}, J=6.0,0.5 \mathrm{~Hz}), 0.95(3 \mathrm{H}, \mathrm{t}, J$ $=7.5 \mathrm{~Hz}) ;{ }^{13} \mathrm{C} \mathrm{NMR}\left(100 \mathrm{MHz}, \mathrm{CDCl}_{3}\right) \delta 143.4(\mathrm{~d}, J=3.5 \mathrm{~Hz}), 141.0,134.1,129.5(\mathrm{~d}, J=3.5 \mathrm{~Hz})$, 128.8, 126.2, $71.6(\mathrm{~d}, J=8.0 \mathrm{~Hz}), 47.0(2 \mathrm{~d}, J=13.5 \mathrm{~Hz}), 31.4(2 \mathrm{~d}, J=9.5 \mathrm{~Hz}), 22.9(\mathrm{~d}, J=6.5 \mathrm{~Hz})$, 21.5, 21.1, 14.2; ${ }^{31} \mathrm{P}$ NMR (121 MHz, $\mathrm{CDCl}_{3}$ ) $\delta$ 23.96; MS (EI) m/e (rel intensity) 385 (13), 356 (20), 304 (78), 303 (76), 239 (94), 230 (76), 188 (91), 148 (100), 133 (95), 91 (44); HRMS (EI) m/e calcd $\left(\mathrm{M}^{+}\right)$385.1589, found 385.1586.

Preparation of diphenylphosphino iminodiazaphospholidines. In a flame dried round bottom flask under $\mathrm{N}_{2}$, allylic alcohol $(0.6 \mathrm{mmol})$ was added to a stirring solution of phospholidine 13 (0.6 mmol) in benzene $(6 \mathrm{~mL})$. The reaction was stirred for 6 hours under a dynamic atmosphere of $\mathrm{N}_{2}$ then checked for completion by ${ }^{31} \mathrm{P}$ NMR. Diphenylphosphoryl azide $(0.6 \mathrm{mmol})$ was then slowly added to the stirring solution resulting in moderate effervescence. After azide addition, the reaction was stirred for 30 min and concentrated in vacuo to yield a crude syrup. The crude syrup was then dissolved in a minimum amount of $\mathrm{CH}_{2} \mathrm{Cl}_{2}$ and purified by column chromatography.

\section{(E)-(1,3-Dimethyl-2-pent-2-enyloxy-2 $\lambda^{5}$-[1,3,2]diazaphospholidin-2-ylidene)-phosphoramidic acid} diphenyl ester (12c).

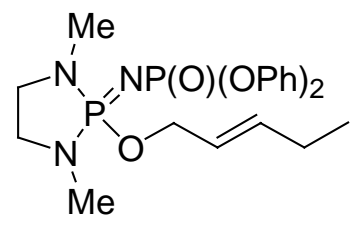

Flash chromatography (EtOAc:hexanes; $4: 1+5 \% \mathrm{NEt}_{3}$ ) yielded a clear oil $(87 \%)$. $\mathrm{R}_{f}=0.21$ (EtOAc:hexanes; 5:1); IR (Thin Film) v 2933, 2874, 1594, 1489, 1299, 1204, 1025, 1005, $914 \mathrm{~cm}^{-1} ;{ }^{1} \mathrm{H}$ NMR (300 MHz, $\left.\mathrm{CDCl}_{3}\right) \delta$ 7.32-7.24 (8H, m), 7.11-7.05 (2H, m), $5.71(1 \mathrm{H}, \mathrm{dtt}, J=15.0,6.5,1.0 \mathrm{~Hz})$, $5.47(1 \mathrm{H}, \mathrm{dtt}, J=15.5,6.5,1.5 \mathrm{~Hz}), 4.29(2 \mathrm{H}, \mathrm{ddd}, J=11.0,6.5,1.0 \mathrm{~Hz}), 3.18-3.08(4 \mathrm{H}, \mathrm{m}), 2.49(6 \mathrm{H}$, 
d, $J=10.5 \mathrm{~Hz}), 2.04(2 \mathrm{H}, \mathrm{qdd}, J=6.5,6.5,1.0 \mathrm{~Hz}), 0.98(3 \mathrm{H}, \mathrm{t}, J=6.5 \mathrm{~Hz}) ;{ }^{13} \mathrm{C} \mathrm{NMR}(100 \mathrm{MHz}$, $\left.\mathrm{CDCl}_{3}\right) \delta 152.6(\mathrm{~d}, J=7.5 \mathrm{~Hz}), 137.8,129.4,124.1(\mathrm{~d}, J=5.5 \mathrm{~Hz}), 123.8,120.7(\mathrm{~d}, J=4.5 \mathrm{~Hz}), 68.4(\mathrm{~d}$, $J=7.5 \mathrm{~Hz}), 47.3(\mathrm{~d}, J=13.5 \mathrm{~Hz}), 31.6(\mathrm{~d}, J=4.0 \mathrm{~Hz}), 25.4,13.3 ;{ }^{31} \mathrm{P} \mathrm{NMR}\left(121 \mathrm{MHz}, \mathrm{CDCl}_{3}\right) \delta 24.76$ (d, $J=56.5 \mathrm{~Hz}),-9.58(\mathrm{~d}, J=56.5 \mathrm{~Hz})$.

(Z)-(1,3-Dimethyl-2-pent-2-enyloxy-2 $\lambda^{5}$-[1,3,2]diazaphospholidin-2-ylidene)-phosphoramidic acid diphenyl ester (12d).

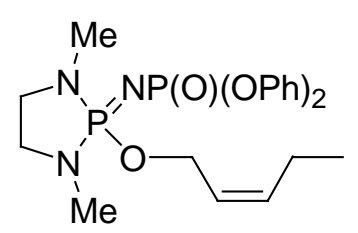

Flash chromatography (EtOAc:hexanes; 4:1 $+5 \% \mathrm{NEt}_{3}$ ) yielded a clear oil $(90 \%) . \mathrm{R}_{f}=0.22$ (EtOAc:hexanes; 5:1); IR (Thin Film) v 2932, 1592, 1489, 1250, 1204, 1004, 915, $768 \mathrm{~cm}^{-1}$; ${ }^{1} \mathrm{H}$ NMR $\left(300 \mathrm{MHz}, \mathrm{CDCl}_{3}\right) \delta$ 7.32-7.23 (8H, m), 7.10-7.02 $(2 \mathrm{H}, \mathrm{m}), 5.59(1 \mathrm{H}, \mathrm{dt}, J=11.0,7.5 \mathrm{~Hz}), 5.43(1 \mathrm{H}$, $\mathrm{dtt}, J=11.0,7.0,1.5 \mathrm{~Hz}), 4.40(2 \mathrm{H}, \mathrm{ddd}, J=11.5,7.5,1.0 \mathrm{~Hz}), 3.18-3.08(4 \mathrm{H}, \mathrm{m}), 2.50(6 \mathrm{H}, \mathrm{d}, J=10.5$ $\mathrm{Hz}), 2.03(2 \mathrm{H}, \mathrm{qd}, J=7.5,6.5 \mathrm{~Hz}), 0.96(3 \mathrm{H}, \mathrm{t}, J=7.5 \mathrm{~Hz}) ;{ }^{13} \mathrm{C} \mathrm{NMR}\left(100 \mathrm{MHz}, \mathrm{CDCl}_{3}\right) \delta 152.6(\mathrm{~d}, J$ $=7.5 \mathrm{~Hz}), 136.7,129.3,124.1(\mathrm{~d}, J=6.0 \mathrm{~Hz}), 123.8,120.7(\mathrm{~d}, J=5.5 \mathrm{~Hz}), 63.2(\mathrm{~d}, J=7.5 \mathrm{~Hz}), 47.3(\mathrm{~d}$, $J=13.5 \mathrm{~Hz}), 31.6(\mathrm{~d}, J=4.0 \mathrm{~Hz}), 21.0,14.3 ;{ }^{31} \mathrm{P} \mathrm{NMR}\left(121 \mathrm{MHz}, \mathrm{CDCl}_{3}\right) \delta 25.02(\mathrm{~d}, J=56.5 \mathrm{~Hz}),-$ $9.56(\mathrm{~d}, J=56.5 \mathrm{~Hz})$.

[1,3-Dimethyl-2-(2-methyl-allyloxy)-2 $\lambda^{5}$-[1,3,2] diazaphospholidin-2-ylidene]-phosphoramidic acid diphenyl ester (12i).

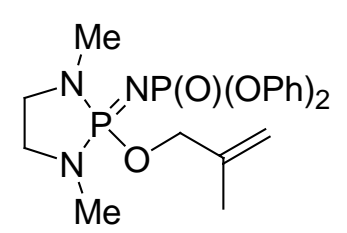

Flash chromatography (EtOAc:hexanes; 4:1 $+5 \% \mathrm{NEt}_{3}$ ) yielded a clear oil $(92 \%) . \mathrm{R}_{f}=0.18$ (EtOAc:hexanes; 4:1); IR (Thin Film) v 2935, 1594, 1490, 1251, 1204, 1013, 914, $772 \mathrm{~cm}^{-1}$; ${ }^{1} \mathrm{H}$ NMR $\left(300 \mathrm{MHz}, \mathrm{CDCl}_{3}\right) \delta$ 7.33-7.23 (8H, m), 7.10-7.02 (2H, m), $4.94(1 \mathrm{H}, \mathrm{s}), 4.88(1 \mathrm{H}, \mathrm{s}), 4.24(2 \mathrm{H}, \mathrm{d}, J=$ $10.0 \mathrm{~Hz}), 3.15(4 \mathrm{H}, \mathrm{m}), 2.50(6 \mathrm{H}, \mathrm{d}, J=10.5 \mathrm{~Hz}), 1.71(3 \mathrm{H}, \mathrm{s}) ;{ }^{13} \mathrm{C} \mathrm{NMR}\left(100 \mathrm{MHz}, \mathrm{CDCl}_{3}\right) \delta 152.5(\mathrm{~d}$, $J=7.5 \mathrm{~Hz}), 140.7(\mathrm{~d}, J=6.0 \mathrm{~Hz}), 129.3,123.8,120.7(\mathrm{~d}, J=4.5 \mathrm{~Hz}), 113.0,70.8(\mathrm{~d}, J=7.5 \mathrm{~Hz}), 47.2$ $(\mathrm{d}, J=14.0 \mathrm{~Hz}), 31.6(\mathrm{~d}, J=4.5 \mathrm{~Hz}), 19.2 ;{ }^{31} \mathrm{P} \mathrm{NMR}\left(121 \mathrm{MHz}, \mathrm{CDCl}_{3}\right) \delta 24.61(\mathrm{~d}, J=56.5 \mathrm{~Hz}),-9.60$ (d, $J=57.0 \mathrm{~Hz}$ ); MS (EI) m/e (rel intensity) 381 (28), 380 (100), 296 (10), 133 (16), 131 (12), 85 (13); HRMS (EI) $m / e$ calcd $\left(\mathrm{M}^{+}\right) 436.1555$, found 436.1545. 


\section{[1,3-Dimethyl-2-(1-methyl-allyloxy)-2 $\lambda^{5}$-[1,3,2]diazaphospholidin-2-ylidene]-phosphoramidic acid}

diphenyl ester (12j).

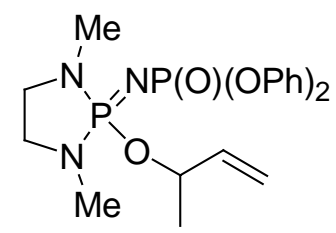

Flash chromatography (EtOAc:hexanes; 4:1 + 5\% $\mathrm{NEt}_{3}$ ) yielded a clear oil $(89 \%)$. $\mathrm{R}_{f}=0.21$ (EtOAc:hexanes; 4:1); IR (Thin Film) v 2931, 1594, 1489, 1250, 1204, 1005, 914, 772, $691 \mathrm{~cm}^{-1} ;{ }^{1} \mathrm{H}$ NMR (300 MHz, $\left.\mathrm{CDCl}_{3}\right) \delta$ 7.32-7.24 (8H, m), 7.11-7.03 (2H, m), $5.78(1 \mathrm{H}, \mathrm{ddd}, J=17.0,10.5,6.5$ $\mathrm{Hz}), 5.13(1 \mathrm{H}, \mathrm{dt}, J=17.0,1.5 \mathrm{~Hz}), 5.07(1 \mathrm{H}, \mathrm{dt}, J=10.5,1.5 \mathrm{~Hz}), 4.67(1 \mathrm{H}, \mathrm{m}), 3.18-3.06(4 \mathrm{H}, \mathrm{m})$, $2.46(6 \mathrm{H}, \mathrm{dd}, J=10.5,2.0 \mathrm{~Hz}), 1.25(3 \mathrm{H}, \mathrm{d}, J=6.5 \mathrm{~Hz}) ;{ }^{13} \mathrm{C} \mathrm{NMR}\left(100 \mathrm{MHz}, \mathrm{CDCl}_{3}\right) \delta 152.6(\mathrm{~d}, J=$ $8.5 \mathrm{~Hz}), 138.8(\mathrm{~d}, J=5.5 \mathrm{~Hz}), 129.3,123.8,120.7(\mathrm{~d}, J=5.5 \mathrm{~Hz}), 115.8,75.5(\mathrm{~d}, J=7.5 \mathrm{~Hz}), 47.2(2 \mathrm{~d}$, $J=12.0,12.0 \mathrm{~Hz}), 31.5(2 \mathrm{~d}, J=4.5,4.5 \mathrm{~Hz}), 22.2(\mathrm{~d}, J=5.5 \mathrm{~Hz}) ;{ }^{31} \mathrm{P} \mathrm{NMR}\left(121 \mathrm{MHz}, \mathrm{CDCl}_{3}\right) \delta 23.51$ (d, $J=55.5 \mathrm{~Hz}),-9.71(\mathrm{~d}, J=56.5 \mathrm{~Hz}$ ); MS (EI) m/e (rel intensity) 408 (10), 380 (100), 323 (20), 133 (20), 131 (24), 85 (34), 77 (21); HRMS (EI) m/e calcd $\left(\mathrm{M}^{+}\right)$436.1555, found 436.1556.

\section{[2-(Cyclohex-2-enyloxy)-1,3-dimethyl-2 $\lambda^{5}$-[1,3,2] diazaphospholidin-2-ylidene]-phosphoramidic} acid diphenyl ester (12k).

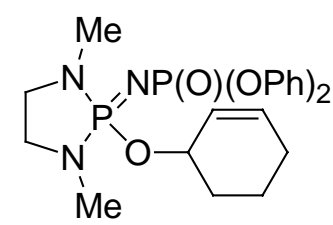

Flash chromatography (EtOAc:hexanes; 4:1 + 5\% $\mathrm{NEt}_{3}$ ) yielded a clear oil $(89 \%) . \mathrm{R}_{f}=0.20$ (EtOAc:hexanes; 4:1); IR (Thin Film) v 2934, 1594, 1489, 1251, 1204, 1162, 913, 773, $691 \mathrm{~cm}^{-1} ;{ }^{1} \mathrm{H}$ NMR (300 MHz, $\left.\mathrm{CDCl}_{3}\right)$ א 7.34-7.24 (8H, m), 7.11-7.03 (2H, m), $5.87(1 \mathrm{H}, \mathrm{dt}, J=10.0,3.5 \mathrm{~Hz}), 5.57$ (1H, ddt, $J=9.0,3.5,1.5 \mathrm{~Hz}), 4.72-4.60(1 \mathrm{H}, \mathrm{m}), 3.20-3.06(4 \mathrm{H}, \mathrm{m}), 2.49(6 \mathrm{H}, \mathrm{dd}, J=10.5,1.0 \mathrm{~Hz})$, 2.10-1.94 (2H, m), 1.80-1.46 (4H, m); $\left.{ }^{13} \mathrm{C} \mathrm{NMR} \mathrm{(100} \mathrm{MHz,} \mathrm{CDCl}_{3}\right) \delta 152.6(\mathrm{~d}, J=8.5 \mathrm{~Hz}), 132.5$, $129.3,126.7,123.8,120.7(\mathrm{~d}, J=5.5 \mathrm{~Hz}), 72.3(\mathrm{~d}, J=8.5 \mathrm{~Hz}), 47.2(2 \mathrm{~d}, J=14.0,14.0 \mathrm{~Hz}), 31.5(2 \mathrm{~d}, J$ $=6.0,6.0 \mathrm{~Hz}), 30.2(\mathrm{~d}, J=6.0 \mathrm{~Hz}), 24.9,18.9 ;{ }^{31} \mathrm{P} \mathrm{NMR}\left(121 \mathrm{MHz}, \mathrm{CDCl}_{3}\right) \delta 23.74(\mathrm{~d}, J=54.5 \mathrm{~Hz}),-$ $9.73(\mathrm{~d}, J=53.5 \mathrm{~Hz}$ ); MS (EI) m/e (rel intensity) 461 (6), 381 (41), 380 (100), 325 (41), 288 (32), 133 (18), 131 (14), 77 (13); HRMS (EI) m/e calcd $\left(\mathrm{M}^{+}\right)$461.1632, found 461.1633. 


\section{(E)-[2-(1-Ethyl-but-2-enyloxy)-1,3-dimethyl-2 $\lambda^{5}$-[1,3,2]diazaphospholidin-2-ylidene]-}

phosphoramidic acid diphenyl ester (12l).

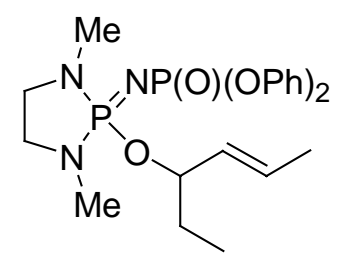

Flash chromatography (EtOAc:hexanes; 4:1 $+5 \% \mathrm{NEt}_{3}$ ) yielded a clear oil $(86 \%) . \mathrm{R}_{f}=0.20$ (EtOAc:hexanes; 4:1); IR (Thin Film) v 2934, 1594, 1490, 1251, 1205, 1162, 989, 912, $771 \mathrm{~cm}^{-1} ;{ }^{1} \mathrm{H}$ NMR (300 MHz, $\left.\mathrm{CDCl}_{3}\right)$ 8 7.34-7.22 (8H, m), 7.10-7.02 (2H, m), $5.52(1 \mathrm{H}, \mathrm{dq}, J=15.0,6.5 \mathrm{~Hz}), 5.35$ $(1 \mathrm{H}, \mathrm{ddd}, J=15.0,8.0,1.0 \mathrm{~Hz}), 4.39(1 \mathrm{H}, \mathrm{ddt}, J=9.5,7.5,7.0 \mathrm{~Hz}), 3.17-3.04(4 \mathrm{H}, \mathrm{m}), 2.46(6 \mathrm{H}, \mathrm{d}, J=$ $11.0 \mathrm{~Hz}), 1.64(3 \mathrm{H}, \mathrm{dd}, J=6.5,1.0 \mathrm{~Hz}), 1.63-1.40(2 \mathrm{H}, \mathrm{m}), 0.83(3 \mathrm{H}, \mathrm{t}, J=7.5 \mathrm{~Hz}) ;{ }^{13} \mathrm{C}$ NMR $(100$ $\left.\mathrm{MHz}, \mathrm{CDCl}_{3}\right) \delta 157.7(\mathrm{~d}, J=7.0 \mathrm{~Hz}), 130.9(\mathrm{~d}, J=3.0 \mathrm{~Hz}), 129.5,129.3,123.8,120.8(\mathrm{~d}, J=5.5 \mathrm{~Hz})$, $81.2(\mathrm{~d}, J=8.5 \mathrm{~Hz}), 47.3(2 \mathrm{~d}, J=14.5,14.5 \mathrm{~Hz}), 31.7(2 \mathrm{~d}, J=6.5,6.5 \mathrm{~Hz}), 29.3(\mathrm{~d}, J=6.0 \mathrm{~Hz}), 17.8$, 9.8; ${ }^{31} \mathrm{P}$ NMR $\left(121 \mathrm{MHz}, \mathrm{CDCl}_{3}\right) \delta 23.69(\mathrm{~d}, J=55.0 \mathrm{~Hz}),-9.67(\mathrm{~d}, J=54.0 \mathrm{~Hz})$; MS (EI) m/e (rel intensity) 381 (34), 380 (100), 325 (29), 323 (37), 85 (21); HRMS (EI) m/e calcd (M+) 463.1790, found 463.1795 .

(E)-[1,3-Dimethyl-2-(3-phenyl-allyloxy)-215-[1,3,2]diazaphospholidin-2-ylidene]-phosphoramidic acid diphenyl ester (12m).

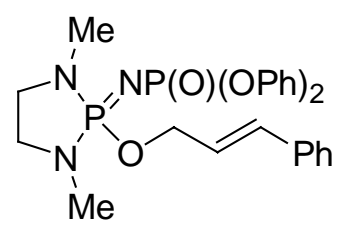

Flash chromatography (EtOAc:hexanes; 4:1 + 5\% $\mathrm{NEt}_{3}$ ) yielded a clear oil $(91 \%) . \mathrm{R}_{f}=0.30$ (EtOAc:hexanes; 5:1); IR (Thin Film) v 3061, 2935, 1594, 1489, 1204, 1163, 915, $747 \mathrm{~cm}^{-1}$; ${ }^{1} \mathrm{H}$ NMR

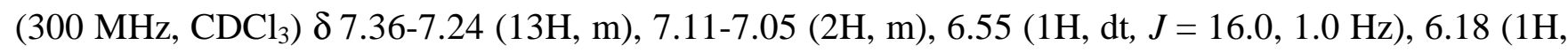
$\mathrm{dt}, J=16.0,6.5 \mathrm{~Hz}), 4.51(2 \mathrm{H}, \mathrm{ddd}, J=12.0,6.5,1.5 \mathrm{~Hz}), 3.20-3.11(4 \mathrm{H}, \mathrm{m}), 2.51(6 \mathrm{H}, \mathrm{d}, J=10.5 \mathrm{~Hz})$; ${ }^{13} \mathrm{C}$ NMR $\left(100 \mathrm{MHz}, \mathrm{CDCl}_{3}\right) \delta 152.6(\mathrm{~d}, J=7.0 \mathrm{~Hz}), 136.2,133.9,129.4,128.8,128.3,126.8,124.3(\mathrm{~d}$, $J=6.0 \mathrm{~Hz}), 123.9,120.7(\mathrm{~d}, J=5.5 \mathrm{~Hz}), 68.2(\mathrm{~d}, J=7.5 \mathrm{~Hz}), 47.3(\mathrm{~d}, J=14.0 \mathrm{~Hz}), 31.7(\mathrm{~d}, J=4.0$ $\mathrm{Hz}) ;{ }^{31} \mathrm{P}$ NMR $\left(121 \mathrm{MHz}, \mathrm{CDCl}_{3}\right) \delta 24.85(\mathrm{~d}, J=56.5 \mathrm{~Hz}),-9.64(\mathrm{~d}, J=56.5 \mathrm{~Hz})$.

(E)-[1,3-Dimethyl-2-(1-methyl-3-phenyl-allyloxy)-215-[1,3,2]diazaphospholidin-2-ylidene]phosphoramidic acid diphenyl ester (12n). 


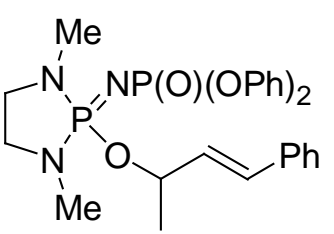

Flash chromatography (EtOAc:hexanes; 4:1 $+5 \% \mathrm{NEt}_{3}$ ) yielded a clear oil $(84 \%) . \mathrm{R}_{f}=0.20$ (EtOAc:hexanes; 4:1); IR (Thin Film) v 2934, 1594, 1489, 1251, 1204, 1162, 913, 773, $691 \mathrm{~cm}^{-1}$; ${ }^{1} \mathrm{H}$ NMR (300 MHz, $\left.\mathrm{CDCl}_{3}\right) \delta$ 7.36-7.22 (13H, m), 7.10-7.04 (2H, m), $6.44(1 \mathrm{H}, \mathrm{d}, J=16.0 \mathrm{~Hz}), 6.11(1 \mathrm{H}$, d, $J=16.0,7.0 \mathrm{~Hz}), 4.87(1 \mathrm{H}, \mathrm{ddq}, J=12.5,7.0,6.5 \mathrm{~Hz}), 3.18-3.09(4 \mathrm{H}, \mathrm{m}), 2.48(3 \mathrm{H}, \mathrm{d}, J=10.5 \mathrm{~Hz})$, $2.46(3 \mathrm{H}, \mathrm{d}, J=10.5 \mathrm{~Hz}), 1.36(3 \mathrm{H}, \mathrm{d}, J=6.5 \mathrm{~Hz}) ;{ }^{13} \mathrm{C} \mathrm{NMR}\left(100 \mathrm{MHz}, \mathrm{CDCl}_{3}\right) \delta 152.7(\mathrm{~d}, J=7.5$ $\mathrm{Hz}), 136.3,131.6,129.9$ (d, $J=4.0 \mathrm{~Hz}), 129.4,128.8,128.2,126.8,123.9$ (d, $J=2.5 \mathrm{~Hz}), 120.8$ (d, $J=$ $3.0 \mathrm{~Hz}), 75.8(\mathrm{~d}, J=8.5 \mathrm{~Hz}), 47.3(2 \mathrm{~d}, J=13.5,14.5 \mathrm{~Hz}), 31.7(2 \mathrm{~d}, J=4.5,4.5 \mathrm{~Hz}), 22.7(\mathrm{~d}, J=6.0$ $\mathrm{Hz}) ;{ }^{31} \mathrm{P}$ NMR $\left(121 \mathrm{MHz}, \mathrm{CDCl}_{3}\right) \delta 23.62(\mathrm{~d}, J=55.0 \mathrm{~Hz}),-9.72(\mathrm{~d}, J=55.0 \mathrm{~Hz})$; MS (EI) $m / e(\mathrm{rel}$ intensity) 512 (14), 511 (42), 380 (90), 325 (87), 294 (30), 288 (57), 131 (89), 130 (90), 129 (100), 115 (72), 85 (31), 77 (47), 51 (31); HRMS (EI) m/e calcd ( $\left.\mathrm{M}^{+}\right)$511.1788, found 511.1790.

\section{[1,3-Dimethyl-2-(3-methyl-but-2-enyloxy)-215-[1,3,2]diazaphospholidin-2-ylidene]-} phosphoramidic acid diphenyl ester (12p).

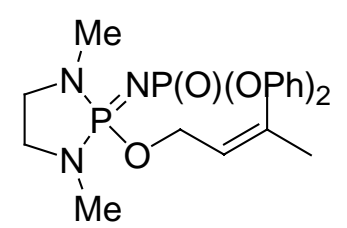

Flash chromatography (EtOAc:hexanes; 4:1 $+5 \% \mathrm{NEt}_{3}$ ) yielded a clear oil $(87 \%) . \mathrm{R}_{f}=0.25$ (EtOAc:hexanes; 5:1); IR (Thin Film) v 2934, 1594, 1490, 1204, 1163, 915, $771 \mathrm{~cm}^{-1}$; ${ }^{1} \mathrm{H}$ NMR (300 $\left.\mathrm{MHz}, \mathrm{CDCl}_{3}\right) \delta 7.34-7.22(8 \mathrm{H}, \mathrm{m}), 7.10-7.03(2 \mathrm{H}, \mathrm{m}), 5.27(1 \mathrm{H}, \mathrm{td}, J=6.0,1.0 \mathrm{~Hz}), 4.53(2 \mathrm{H}, \mathrm{dd}, J=$ 11.0, $7.0 \mathrm{~Hz}), 3.20-3.08(4 \mathrm{H}, \mathrm{m}), 2.49(6 \mathrm{H}, \mathrm{d}, J=10.5 \mathrm{~Hz}), 1.73(3 \mathrm{H}, \mathrm{s}), 1.64(3 \mathrm{H}, \mathrm{s}) ;{ }^{13} \mathrm{C}$ NMR $(100$ $\left.\mathrm{MHz}, \mathrm{CDCl}_{3}\right) \delta 152.6(\mathrm{~d}, J=7.5 \mathrm{~Hz}), 139.0,129.3,123.8,120.7(\mathrm{~d}, J=7.5 \mathrm{~Hz}), 119.9(\mathrm{~d}, J=6.0 \mathrm{z})$, $64.3(\mathrm{~d}, J=5.5 \mathrm{~Hz}), 47.3(\mathrm{~d}, J=13.5 \mathrm{~Hz}), 31.5(\mathrm{~d}, J=4.5 \mathrm{~Hz}), 25.9,18.2 ;{ }^{31} \mathrm{P}$ NMR $\left(121 \mathrm{MHz}, \mathrm{CDCl}_{3}\right)$ $\delta 24.9(\mathrm{~d}, J=55.5 \mathrm{~Hz}$ ), -9.60 (d, $J=55.5 \mathrm{~Hz}$ ); MS (EI) m/e (rel intensity) 449 (7), 381 (35), 380 (100), 325 (39), 288 (30), 133 (22), 85 (26), 67 (24); HRMS (EI) m/e calcd ( $\left.\mathrm{M}^{+}\right)$449.1629, found 449.1633.

\section{(E)-(S)-[1,3-Dimethyl-2-(1-methyl-pent-2-enyloxy)-2 $\lambda^{5}$-[1,3,2]diazaphospholidin-2-ylidene]-} phosphoramidic acid diphenyl ester (12q). 


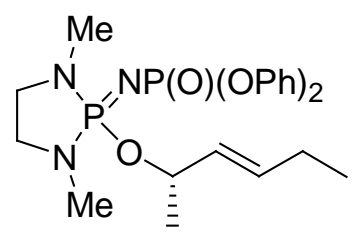

Flash chromatography (EtOAc:hexanes; 4:1 $+5 \% \mathrm{NEt}_{3}$ ) yielded a clear oil $(78 \%) \cdot[\alpha]_{\mathrm{D}}=-11.3^{\circ}$ $\left(\mathrm{CHCl}_{3}, \mathrm{c}=1.2\right) ; \mathrm{R}_{f}=0.24$ (EtOAc:hexanes; 3:1); IR (Thin Film) v 2932, 1447, 1357, 1271, 1163, 1043, 990, $672 \mathrm{~cm}^{-1} ;{ }^{1} \mathrm{H}$ NMR $\left(300 \mathrm{MHz}, \mathrm{CDCl}_{3}\right) \delta$ 7.32-7.24 (8H, m), 7.10-7.03 $(2 \mathrm{H}, \mathrm{m}), 5.57(1 \mathrm{H}, \mathrm{dt}, J=$ 15.5, $6.0 \mathrm{~Hz}$ ), 5.39 (1H, ddt, $J=15.5,7.0,1.5 \mathrm{~Hz}), 4.72-4.58(1 \mathrm{H}, \mathrm{m}), 3.16-3.04(4 \mathrm{H}, \mathrm{m}), 2.46(3 \mathrm{H}, \mathrm{d}, J$ $=10.5 \mathrm{~Hz}), 2.45(3 \mathrm{H}, \mathrm{d}, J=10.5 \mathrm{~Hz}), 1.99(2 \mathrm{H}, \mathrm{qd}, J=7.5,6.5 \mathrm{~Hz}), 1.24(3 \mathrm{H}, \mathrm{d}, J=6.5 \mathrm{~Hz}), 0.95(3 \mathrm{H}$, t, $J=7.5 \mathrm{~Hz}) ;{ }^{13} \mathrm{C}$ NMR $\left(100 \mathrm{MHz}, \mathrm{CDCl}_{3}\right) \delta 152.6(\mathrm{~d}, J=6.5 \mathrm{~Hz}), 134.9,129.7(\mathrm{~d}, J=4.0 \mathrm{~Hz}), 129.3$, $123.7(\mathrm{~d}, J=1.0 \mathrm{~Hz}), 120.7$ (d, $J=4.0 \mathrm{~Hz}), 75.9$ (d, $J=8.0 \mathrm{~Hz}), 47.2(2 \mathrm{~d}, J=13.5,13.5 \mathrm{~Hz}), 31.5(2 \mathrm{~d}$, $J=4.5,4.5 \mathrm{~Hz}), 25.2,22.6(\mathrm{~d}, J=16.5 \mathrm{~Hz}), 13.3 ;{ }^{31} \mathrm{P} \mathrm{NMR}\left(121 \mathrm{MHz}, \mathrm{CDCl}_{3}\right) \delta 23.54(\mathrm{~d}, J=55.5 \mathrm{~Hz})$, $-9.68(\mathrm{~d}, J=55.5 \mathrm{~Hz}$ ); MS (EI) m/e (rel intensity) 463 (5), 381 (42), 382 (100), 325 (45), 288 (35), 133 (21), 85 (25); HRMS (EI) m/e calcd ( $\left.\mathrm{M}^{+}\right) 463.1790$, found 463.1783.

\section{(Z)-(S)-[1,3-Dimethyl-2-(1-methyl-pent-2-enyloxy)-2 $\lambda^{5}$-[1,3,2] diazaphospholidin-2-ylidene]-} phosphoramidic acid diphenyl ester (12r).

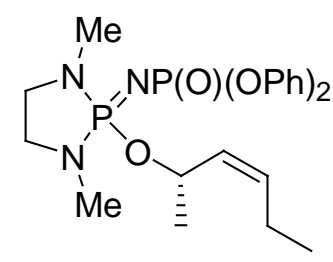

Flash chromatography (EtOAc:hexanes; 4:1 $+5 \% \mathrm{NEt}_{3}$ ) yielded a clear oil $(80 \%) \cdot[\alpha]_{\mathrm{D}}=+11.1^{\circ}$ $\left(\mathrm{CHCl}_{3}, \mathrm{c}=2.1\right) ; \mathrm{R}_{f}=0.26$ (EtOAc:hexanes; 3:1); IR (Thin Film) v 2932, 1447, 1357, 1271, 1163, 1043, 990, $672 \mathrm{~cm}^{-1}$; ${ }^{1} \mathrm{H}$ NMR $\left(300 \mathrm{MHz}, \mathrm{CDCl}_{3}\right) \delta$ 7.32-7.23 (8H, m), 7.10-7.04 (2H, m), $5.41(1 \mathrm{H}, \mathrm{dt}, J=$ 11.0, 7.5 Hz), $5.35(1 \mathrm{H}, \mathrm{dd}, J=11.0,9.0 \mathrm{~Hz}), 5.10-5.00(1 \mathrm{H}, \mathrm{m}), 3.17-3.34(4 \mathrm{H}, \mathrm{m}), 2.48(3 \mathrm{H}, \mathrm{d}, J=$ $10.5 \mathrm{~Hz}), 2.47(3 \mathrm{H}, \mathrm{d}, J=10.5 \mathrm{~Hz}), 2.00(2 \mathrm{H}, \mathrm{dq}, J=7.5,7.5 \mathrm{~Hz}), 1.23(3 \mathrm{H}, \mathrm{d}, J=6.0 \mathrm{~Hz}), 0.94(3 \mathrm{H}, \mathrm{t}$, $J=7.5 \mathrm{~Hz}) ;{ }^{13} \mathrm{C}$ NMR $\left(100 \mathrm{MHz}, \mathrm{CDCl}_{3}\right) \delta 152.6(\mathrm{~d}, J=7.5 \mathrm{~Hz}), 133.6,130.0(\mathrm{~d}, J=4.5 \mathrm{~Hz}), 129.3$, $123.7(\mathrm{~d}, J=3.5 \mathrm{~Hz}), 120.6(2 \mathrm{~d}, J=6.0 \mathrm{~Hz}), 71.2(\mathrm{~d}, J=8.0 \mathrm{~Hz}), 47.2(2 \mathrm{~d}, J=13.5,13.5 \mathrm{~Hz}), 31.5(2 \mathrm{~d}$, $J=4.5,4.5 \mathrm{~Hz}), 23.0(\mathrm{~d}, J=6.0 \mathrm{~Hz}), 21.1,14.3 ;{ }^{31} \mathrm{P} \mathrm{NMR}\left(121 \mathrm{MHz}, \mathrm{CDCl}_{3}\right) \delta 23.47(\mathrm{~d}, J=55.5 \mathrm{~Hz})$, -9.74 (d, $J=55.5 \mathrm{~Hz}$ ) ; MS (EI) m/e (rel intensity) 463 (3), 381 (34), 380 (100), 325 (22), 296 (14), 288 (31), 270 (11); HRMS (EI) m/e calcd $\left(\mathrm{M}^{+}\right)$463.1790, found 463.1802. 
Rearrangement of tosyl iminodiazaphospholidines without heat (Method A). In a flame dried round bottom flask under $\mathrm{N}_{2}$, a solution of iminodiazaphospholidine $(0.6 \mathrm{mmol})$ in $\mathrm{CH}_{2} \mathrm{Cl}_{2}(5 \mathrm{~mL})$ was added to $8 \mathrm{mg}$ of bis(acetonitrile)dichloropalladium(II) $(5 \mathrm{~mol} \%)$. The reaction was stirred for 16 hours under an atmosphere of $\mathrm{N}_{2}$ then checked for completion by ${ }^{31} \mathrm{P}$ NMR. The reaction was then concentrated in vacuo, dissolved in a minimum amount of $\mathrm{CH}_{2} \mathrm{Cl}_{2}$, and purified by column chromatography.

Rearrangement of tosyl iminodiazaphospholidines with heat (Method B). In a flame dried round bottom flask fitted with a reflux condenser under $\mathrm{N}_{2}$, a solution of iminodiazaphospholidine (0.6 mmol) in $\mathrm{CH}_{2} \mathrm{Cl}_{2}(5 \mathrm{~mL})$ was added to $8 \mathrm{mg}$ of bis(acetonitrile)dichloropalladium(II) (5 mol\%) and freshly activated $4 \AA$ sieves $(50 \mathrm{mg})$. The reaction was heated to $45^{\circ} \mathrm{C}$, stirred for 16 hours under an atmosphere of $\mathrm{N}_{2}$, and checked for completion by ${ }^{31} \mathrm{P}$ NMR. The reaction was then cooled to room temperature, concentrated in vacuo, dissolved in a minimum amount of $\mathrm{CH}_{2} \mathrm{Cl}_{2}$, and purified by column chromatography.

Asymmetric Rearrangement of tosyl iminodiazaphospholidines (Method C). In a flame dried conical vial under $\mathrm{N}_{2}$, toluene $(0.3 \mathrm{~mL})$ was added to a solid mixture of iminodiazaphospholidine $(0.3$ mmol), $11 \mathrm{mg}$ of $\mathrm{COPCl}(0.015 \mathrm{mmol}$ of $\mathrm{Pd})$, and $11 \mathrm{mg}$ of AgTFA (0.03 mmol). The reaction was sealed heated to $45^{\circ} \mathrm{C}$, stirred for 40 hours and checked for completion by ${ }^{31} \mathrm{P}$ NMR. The brown reaction mixture was then cooled to room temperature, concentrated in vacuo, dissolved in a minimum amount of $\mathrm{CH}_{2} \mathrm{Cl}_{2}$, and purified by column chromatography.

\section{$N$-(1,3-Dimethyl-2-oxo-2 $\lambda^{5}$-[1,3,2]diazaphospholidin-2-yl)-4-methyl- $N$-(1-vinyl-butyl)-} benzenesulfonamide (16a).

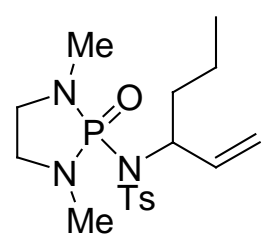

Flash chromatography (EtOAc:hexanes; 2:1) yielded a clear oil. Method A from trans 11a: 91\%. Method A from cis 11b: 85\%. Method C from cis 11b: $90 \%, 96 \%$ ee in favour of $R$ isomer, $[\alpha]_{\mathrm{D}}=$ $+18.9^{\circ}\left(\mathrm{CHCl}_{3}, \mathrm{c}=2.9\right)$. Method C: from trans 11a: $55 \%, 86 \%$ ee in favour of $S$ isomer, $[\alpha]_{\mathrm{D}}=-16.4^{\circ}$ $\left(\mathrm{CHCl}_{3}, \mathrm{c}=2.0\right) ; \mathrm{R}_{f}=0.36$ (EtOAc:hexanes; $\left.2: 1\right) ;{ }^{1} \mathrm{H} \mathrm{NMR}\left(300 \mathrm{MHz}, \mathrm{CDCl}_{3}\right) \delta 7.76(2 \mathrm{H}, \mathrm{d}, J=8.5$ $\mathrm{Hz}), 7.31(2 \mathrm{H}, \mathrm{d}, J=8.0 \mathrm{~Hz}), 6.25(1 \mathrm{H}, \mathrm{ddd}, J=17.5,10.0,8.5 \mathrm{~Hz}), 4.98(1 \mathrm{H}, \mathrm{d}, J=10.0 \mathrm{~Hz}), 4.76$ $(1 \mathrm{H}, \mathrm{d}, J=17.0 \mathrm{~Hz}), 4.22-4.02(1 \mathrm{H}, \mathrm{m}), 3.50-3.14(4 \mathrm{H}, \mathrm{m}), 2.58(3 \mathrm{H}, \mathrm{d}, J=10.0 \mathrm{~Hz}), 2.44(3 \mathrm{H}, \mathrm{s}), 2.42$ 
(3H, d, $J=10.0 \mathrm{~Hz}), 2.24-2.10(1 \mathrm{H}, \mathrm{m}), 1.63-1.47(1 \mathrm{H}, \mathrm{m}), 1.32-1.04(2 \mathrm{H}, \mathrm{m}), 0.83(3 \mathrm{H}, \mathrm{t}, J=7.0 \mathrm{~Hz})$; ${ }^{13} \mathrm{C}$ NMR $\left(100 \mathrm{MHz}, \mathrm{CDCl}_{3}\right) \delta 143.9(\mathrm{~d}, J=14.5 \mathrm{~Hz}), 139.1,138.4,129.5,128.0,118.0,63.9,46.6$, 37.0, $31.3(\mathrm{~d}, J=5.0 \mathrm{~Hz}), 21.7,20.3,13.8 ;{ }^{31} \mathrm{P}$ NMR $\left(121 \mathrm{MHz}, \mathrm{CDCl}_{3}\right) \delta 19.51$; MS (EI) m/e (rel intensity) 386 (7), 321 (8), 304 (9), 278 (28), 230 (36), 188 (24), 149 (13), 133 (100); HRMS (EI) m/e calcd $\left(\mathrm{M}^{+}\right)$385.1589, found 385.1578.

\section{$N$-(1,3-Dimethyl-2-oxo-2 $\lambda^{5}$-[1,3,2] diazaphospholidin-2-yl)- $N$-(1-ethyl-allyl)-4-methyl-} benzenesulfonamide (16c).

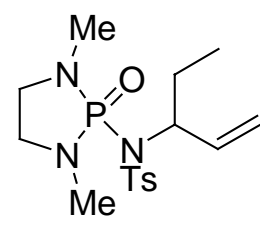

Flash chromatography (EtOAc:hexanes; 2:1) yielded a clear oil. Method A from trans 11c: 95\%. Method A from cis 11d: 93\%. Method C from cis 11d: 92\%, 93\% ee in favour of $R$ isomer, $[\alpha]_{\mathrm{D}}=$ $+22.3^{\circ}\left(\mathrm{CHCl}_{3}, \mathrm{c}=1.5\right)$. Method $\mathrm{C}$ : from trans 11c: $60 \%, 84 \%$ ee in favour of $S$ isomer, $[\alpha]_{\mathrm{D}}=-19.5^{\circ}$ $\left(\mathrm{CHCl}_{3}, \mathrm{c}=1.0\right) ; \mathrm{R}_{f}=0.38$ (EtOAc:hexanes; 3:1); IR (Thin Film) v 2933, 1382, 1342, 1267, 1235, 1165, 1070, 928, 743, $667 \mathrm{~cm}^{-1} ;{ }^{1} \mathrm{H}$ NMR $\left(300 \mathrm{MHz}, \mathrm{CDCl}_{3}\right) \delta 7.76(2 \mathrm{H}, \mathrm{d}, J=8.5 \mathrm{~Hz}), 7.30(2 \mathrm{H}, \mathrm{d}, J=8.0$ Hz), 6.25 (1H, ddd, $J=17.5,10.0,9.0 \mathrm{~Hz}), 5.99(1 \mathrm{H}, \mathrm{d}, J=10.0 \mathrm{~Hz}), 4.78(1 \mathrm{H}, \mathrm{d}, J=17.5 \mathrm{~Hz}), 4.16-$ $3.98(1 \mathrm{H}, \mathrm{m}), 3.48-3.13(4 \mathrm{H}, \mathrm{m}), 2.58(3 \mathrm{H}, \mathrm{d}, J=10.5 \mathrm{~Hz}), 2.42(3 \mathrm{H}, \mathrm{d}, J=10.5 \mathrm{~Hz}), 2.44(3 \mathrm{H}, \mathrm{s})$, 2.22-2.04 (1H, m), 1.74-1.6 (1H, m), $0.79(3 \mathrm{H}, \mathrm{t}, J=7.5 \mathrm{~Hz}) ;{ }^{13} \mathrm{C} \mathrm{NMR}\left(100 \mathrm{MHz}, \mathrm{CDCl}_{3}\right) \delta 143.7$, 138.3, 129.3, 127.8, 126.1, 118.1, 65.7, 46.4 (2d, $J=12.0,12.0 \mathrm{~Hz}), 31.1(2 \mathrm{~d}, J=5.0,5.0 \mathrm{~Hz}), 27.9$, 21.5, 11.6; ${ }^{31} \mathrm{P}$ NMR (121 MHz, $\mathrm{CDCl}_{3}$ ) $\delta$ 19.47; MS (EI) m/e (rel intensity) 371 (13), 342 (26), 307 (21), 278 (84), 216 (44), 133 (100), 90 (29); HRMS (EI) m/e calcd (M+) 371.1433, found 371.1442.

\section{$N$-[1-(tert-Butyl-dimethyl-silanyloxymethyl)-allyl]- $N$-(1,3-dimethyl-2-oxo-2 $\lambda^{5}$ -}

\section{$[1,3,2]$ diazaphospholidin-2-yl)-4-methyl-benzenesulfonamide (16e).}

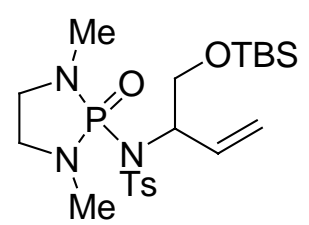

Flash chromatography (EtOAc:hexanes; 2:1) yielded a clear oil. Method A from trans 11e: 88\%. Method A from cis 11f: $91 \%$. Method C from cis 11f: $82 \%, 92 \%$ ee in favour of $R$ isomer, $[\alpha]_{\mathrm{D}}=+15.8^{\circ}$ $\left(\mathrm{CHCl}_{3}, \mathrm{c}=1.6\right)$. Method C: from trans 11e: $44 \%, 86 \%$ ee in favour of $S$ isomer, $[\alpha]_{\mathrm{D}}=-13.8^{\circ}\left(\mathrm{CHCl}_{3}\right.$, $\mathrm{c}=1.5) ; \mathrm{R}_{f}=0.38$ (EtOAc:hexanes; 2:1); IR (Thin Film) v 2928, 2856, 1343, 1257, 1166, 1092, 1038, 
$931 \mathrm{~cm}^{-1} ;{ }^{1} \mathrm{H}$ NMR $\left(300 \mathrm{MHz}, \mathrm{CDCl}_{3}\right) \delta 7.82(2 \mathrm{H}, \mathrm{d}, J=8.5 \mathrm{~Hz}), 7.35(2 \mathrm{H}, \mathrm{d}, J=8.0 \mathrm{~Hz}), 6.28(1 \mathrm{H}$, ddd, $J=18.0,10.0,8.0 \mathrm{~Hz}), 5.10(1 \mathrm{H}, \mathrm{d}, J=10.5 \mathrm{~Hz}), 4.94(1 \mathrm{H}, \mathrm{d}, J=18.0 \mathrm{~Hz}), 4.48-4.32(1 \mathrm{H}, \mathrm{m})$, $4.08(1 \mathrm{H}, \mathrm{dd}, J=9.5,8.0 \mathrm{~Hz}), 3.92(4 \mathrm{H}, \mathrm{dd}, J=10.5,9.5 \mathrm{~Hz}), 3.48-3.20(4 \mathrm{H}, \mathrm{m}), 2.60(3 \mathrm{H}, \mathrm{d}, J=10.5$ $\mathrm{Hz}), 2.48(3 \mathrm{H}, \mathrm{s}), 2.44(3 \mathrm{H}, \mathrm{d}, J=10.5 \mathrm{~Hz}), 0.91(9 \mathrm{H}, \mathrm{s}), 0.07(3 \mathrm{H}, \mathrm{s}), 0.06(3 \mathrm{H}, \mathrm{s}) ;{ }^{13} \mathrm{C} \mathrm{NMR}(100$ $\left.\mathrm{MHz}, \mathrm{CDCl}_{3}\right) \delta 143.8,138.2,137.3,129.4,127.9,119.0,65.5,64.8,46.5(2 \mathrm{~d}, J=14.0 \mathrm{~Hz}), 31.3(2 \mathrm{~d}, J$ $=5.0 \mathrm{~Hz}), 26.0,21.7,18.5,-5.1(\mathrm{~d}, J=14.0 \mathrm{~Hz}) ;{ }^{31} \mathrm{P}$ NMR $\left(121 \mathrm{MHz}, \mathrm{CDCl}_{3}\right) \delta 19.78 ; \mathrm{MS}(\mathrm{EI}) \mathrm{m} / e(\mathrm{rel}$ intensity) 488 (24), 430 (93), 360 (89), 342 (21), 332 (41), 302 (56), 280 (18), 207 (62), 133 (100); HRMS (EI) $m / e$ calcd $\left(\mathrm{M}^{+}\right)$488.2168, found 488.2179.

\section{$N$-(1,3-Dimethyl-2-oxo-2 $\lambda^{5}$-[1,3,2] diazaphospholidin-2-yl)-4-methyl- $N$-(1-methyl-allyl)-} benzenesulfonamide $(16 \mathrm{~g})$.

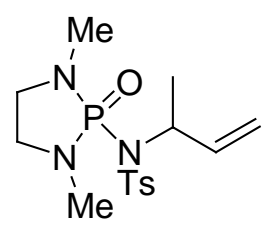

Flash Chromatography (Hex:EtOAc; 1:2) yielded a clear oil. Method A from trans 11g: 88\%. Method A from cis 11h: $86 \%$. Method C from cis 11h: $86 \%, 91 \%$ ee in favour of $R$ isomer, $[\alpha]_{\mathrm{D}}=+14.2^{\circ}\left(\mathrm{CHCl}_{3}\right.$, $\mathrm{c}=1.8)$. Method C: from trans 11g: $45 \%, 86 \%$ ee in favour of $S$ isomer, $[\alpha]_{\mathrm{D}}=-13.5^{\circ}\left(\mathrm{CHCl}_{3}, \mathrm{c}=1.5\right) ; \mathrm{R}_{f}$ $=0.42\left(\right.$ EtOAc:hexanes; 2:1); IR (Thin Film) v 2934, 1342, 1267, 1236, 1165, 1040, $913 \mathrm{~cm}^{-1}{ }^{1} \mathrm{H}_{\mathrm{NMR}}$ $\left(300 \mathrm{MHz}, \mathrm{CDCl}_{3}\right) \delta 7.74(2 \mathrm{H}, \mathrm{d}, J=8.0 \mathrm{~Hz}), 7.29(2 \mathrm{H}, \mathrm{d}, J=8.0 \mathrm{~Hz}), 6.21(1 \mathrm{H}, \mathrm{ddd}, J=17.5,10.5$, $6.5 \mathrm{~Hz}), 4.92(1 \mathrm{H}, \mathrm{d}, J=10.0 \mathrm{~Hz}), 4.80(1 \mathrm{H}, \mathrm{d}, J=17.5 \mathrm{~Hz}), 4.40(1 \mathrm{H}, \mathrm{m}), 3.42-3.28(2 \mathrm{H}, \mathrm{m}), 3.26-3.10$ $(2 \mathrm{H}, \mathrm{m}), 2.52(3 \mathrm{H}, \mathrm{d}, J=10.5 \mathrm{~Hz}), 2.44(3 \mathrm{H}, \mathrm{d}, J=10.5 \mathrm{~Hz}), 2.41(3 \mathrm{H}, \mathrm{s}), 1.45(3 \mathrm{H}, \mathrm{d}, J=7.0 \mathrm{~Hz}) ;{ }^{13} \mathrm{C}$ NMR $\left(100 \mathrm{MHz}, \mathrm{CDCl}_{3}\right) \delta 143.8,140.3,138.0,129.5,127.8,116.2,58.8(\mathrm{~d}, J=2.0 \mathrm{~Hz}), 46.4(2 \mathrm{~d}, J=$ $14.5 \mathrm{~Hz}), 31.2(\mathrm{~d}, J=5.5 \mathrm{~Hz}), 21.7,21.1 ;{ }^{31} \mathrm{P} \mathrm{NMR}\left(121 \mathrm{MHz}, \mathrm{CDCl}_{3}\right) \delta 19.69 ; \mathrm{MS}(\mathrm{ESI}) \mathrm{m} / e$ (rel intensity) 380 (25), 358 (23), 326 (25), 304 (100), 151 (21), 133 (33); HRMS (ESI) m/e calcd $\left(\mathrm{MH}^{+}\right)$ 358.1348 , found 358.1357.

$N$-(1,3-Dimethyl-2-oxo-2 $\lambda^{5}$-[1,3,2] diazaphospholidin-2-yl)-4-methyl- $N$-(2-methyl-allyl)benzenesulfonamide (16i).

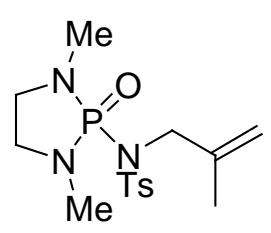


Flash chromatography (EtOAc:hexanes; 2:1) yielded a clear oil (95\%). $\mathrm{R}_{f}=0.37$ (EtOAc:hexanes; 3:1); IR (Thin Film) v 2934, 1598, 1342, 1271, 1235, 1163, 1040, $835 \mathrm{~cm}^{-1} ;{ }^{1} \mathrm{H}$ NMR (300 MHz, $\left.\mathrm{CDCl}_{3}\right)$ $\delta 7.74(2 \mathrm{H}, \mathrm{d}, J=8.5 \mathrm{~Hz}), 7.29(2 \mathrm{H}, \mathrm{d}, J=8.0 \mathrm{~Hz}), 5.03(1 \mathrm{H}, \mathrm{s}), 4.92(1 \mathrm{H}, \mathrm{s}), 4.23(2 \mathrm{H}, \mathrm{d}, J=10.0 \mathrm{~Hz})$, 3.40-3.29 (2H, m), 3.20-3.11 (2H, m), $2.43(3 \mathrm{H}, \mathrm{s}), 2.38(6 \mathrm{H}, \mathrm{d}, J=10.5 \mathrm{~Hz}), 1.75(3 \mathrm{H}, \mathrm{s}) ;{ }^{13} \mathrm{C}$ NMR $\left(100 \mathrm{MHz} \mathrm{CDCl}_{3}\right) \delta 143.8,142.1,138.5,129.4,127.7,113.5,54.6(\mathrm{~d}, J=2.0 \mathrm{~Hz}), 46.6(\mathrm{~d}, J=5.0 \mathrm{~Hz})$, $31.4(\mathrm{~d}, J=4.5 \mathrm{~Hz}), 21.7,20.6 ;{ }^{31} \mathrm{P} \mathrm{NMR}\left(121 \mathrm{MHz}, \mathrm{CDCl}_{3}\right) \delta 19.09 ; \mathrm{MS}$ (EI) m/e (rel intensity) 358 (6), 302 (10), 293 (18), 202 (53), 149 (22), 133 (100), 91 (21); HRMS (EI) m/e calcd (M+) 357.1276, found 357.1280 .

\section{(E)- $N$-But-2-enyl- $N$-(1,3-dimethyl-2-oxo-2 $\lambda^{5}$-[1,3,2]diazaphospholidin-2-yl)-4-methyl-}

\section{benzenesulfonamide (16j).}

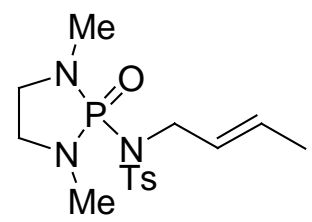

Flash chromatography (EtOAc:hexanes; 2:1) yielded a clear oil (91\%). $\mathrm{R}_{f}=0.44$ (EtOAc:hexanes; 3:1); IR (Thin Film) v 2936, 1346, 1263, 1230, 1164, 1028, $840 \mathrm{~cm}^{-1} ;{ }^{1} \mathrm{H}$ NMR $\left(300 \mathrm{MHz}, \mathrm{CDCl}_{3}\right) \delta 7.74$ $(2 \mathrm{H}, \mathrm{d}, J=8.0 \mathrm{~Hz}), 7.29(2 \mathrm{H}, \mathrm{d}, J=8.5 \mathrm{~Hz}), 5.69(1 \mathrm{H}, \mathrm{dt}, J=15.0,6.5 \mathrm{~Hz}), 5.58(1 \mathrm{H}, \mathrm{dq}, J=15.0,6.5$ Hz), 4.13 (2H, dd, $J=10.5,6.5 \mathrm{~Hz}), 3.38-3.26(2 \mathrm{H}, \mathrm{m}), 3.24-3.12(2 \mathrm{H}, \mathrm{m}), 2.43(3 \mathrm{H}, \mathrm{s}), 2.40(6 \mathrm{H}, \mathrm{d}, J$ $=10.5 \mathrm{~Hz}), 1.63(3 \mathrm{H}, \mathrm{dd}, J=6.5,1.0 \mathrm{~Hz}) ;{ }^{13} \mathrm{C} \mathrm{NMR}\left(100 \mathrm{MHz}, \mathrm{CDCl}_{3}\right) \delta 143.7,138.5,130.3,129.4$, 128.2, 127.6, $51.1(\mathrm{~d}, J=2.3 \mathrm{~Hz}), 46.5(\mathrm{~d}, J=14.5 \mathrm{~Hz}), 31.2(\mathrm{~d}, J=4.6 \mathrm{~Hz}), 21.7,17.8 ;{ }^{31} \mathrm{P}$ NMR $(121$ $\mathrm{MHz}, \mathrm{CDCl}_{3}$ ) $\delta$ 18.87; MS (EI) m/e (rel intensity) 357 (15), 293 (19), 202 (98), 160 (23), 133 (100), 91 (21); HRMS (EI) m/e calcd $\left(\mathrm{M}^{+}\right)$357.1276, found 357.1266.

\section{$N$-Cyclohex-2-enyl- $N$-(1,3-dimethyl-2-oxo-2 $\lambda^{5}$-[1,3,2]diazaphospholidin-2-yl)-4-methyl-} benzenesulfonamide (16k).

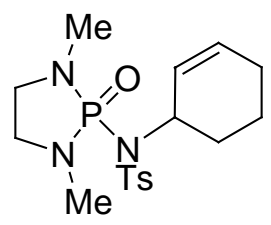

Flash chromatography (EtOAc:hexanes; 2:1) yielded a clear oil (88\%). $\mathrm{R}_{f}=0.38$ (EtOAc:hexanes; 3:1); IR (Thin Film) v 2936, 1346, 1267, 1230, 1168, 1028, $842 \mathrm{~cm}^{-1} ;{ }^{1} \mathrm{H}$ NMR $\left(300 \mathrm{MHz}, \mathrm{CDCl}_{3}\right) \delta 7.76$ $(2 \mathrm{H}, \mathrm{d}, J=8.5 \mathrm{~Hz}), 7.31(2 \mathrm{H}, \mathrm{d}, J=8.0 \mathrm{~Hz}), 5.64(1 \mathrm{H}, \mathrm{bm}), 5.30(1 \mathrm{H}, \mathrm{bd}, J=10.0 \mathrm{~Hz}), 4.51(1 \mathrm{H}, \mathrm{bm})$, 3.44-3.36 (2H, m), 3.24-3.15 (2H, m), $2.58(3 \mathrm{H}, \mathrm{d}, J=10.5 \mathrm{~Hz}), 2.48(3 \mathrm{H}, \mathrm{d}, J=10.5 \mathrm{~Hz}), 2.44(3 \mathrm{H}, \mathrm{s})$, 
2.2-1.5 (6H, m); ${ }^{13} \mathrm{C}$ NMR (100 MHz, $\left.\mathrm{CDCl}_{3}\right) \delta 143.9,129.6,129.5,128.4,127.6,58.1,46.5(2 \mathrm{~d}, J=$ 14.5, 14.5 Hz), $31.4(2 \mathrm{~d}, J=4.5,4.5 \mathrm{~Hz}), 29.6,24.1,23.2,21.7$ (one aromatic C not observed); ${ }^{31} \mathrm{P}$ NMR (121 MHz, $\mathrm{CDCl}_{3}$ ) $\delta$ 19.29; MS (EI) m/e (rel intensity) 383 (4), 304 (12), 228 (100), 151 (21), 133 (70), 91 (16); HRMS (EI) m/e calcd $\left(\mathrm{M}^{+}\right)$383.1433, found 383.1424.

(E)-N-(1,3-Dimethyl-2-oxo-2 $\lambda^{5}$-[1,3,2] diazaphospholidin-2-yl)-4-methyl-N-(1-methyl-pent-2-enyl)benzenesulfonamide (161).

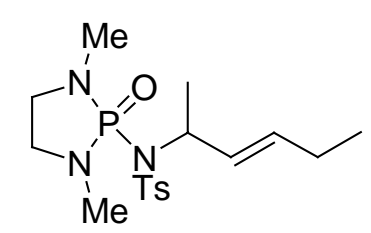

Flash chromatography (EtOAc:hexanes; 2:1) yielded a clear oil (90\%). $\mathrm{R}_{f}=0.31$ (EtOAc:hexanes; 3:1); ${ }^{1} \mathrm{H}$ NMR $\left(300 \mathrm{MHz}, \mathrm{CDCl}_{3}\right) \delta 7.76(2 \mathrm{H}, \mathrm{d}, J=8.0 \mathrm{~Hz}), 7.29(2 \mathrm{H}, \mathrm{d}, J=8.0 \mathrm{~Hz}), 5.91(1 \mathrm{H}, \mathrm{dd}, J=15.5$, $8.0 \mathrm{~Hz}), 5.18(1 \mathrm{H}, \mathrm{dt}, J=15.0,7.0 \mathrm{~Hz}), 4.39(1 \mathrm{H}, \mathrm{ddq}, J=14.0,8.0,7.0 \mathrm{~Hz}), 3.44-3.12(4 \mathrm{H}, \mathrm{m}), 2.54$ $(3 \mathrm{H}, \mathrm{d}, J=10.5 \mathrm{~Hz}), 2.46(3 \mathrm{H}, \mathrm{d}, J=10.5 \mathrm{~Hz}), 2.43(3 \mathrm{H}, \mathrm{s}), 1.89(2 \mathrm{H}, \mathrm{qd}, J=7.5,6.5 \mathrm{~Hz}), 1.46(3 \mathrm{H}, \mathrm{d}$, $J=7.0 \mathrm{~Hz}), 0.83(3 \mathrm{H}, \mathrm{t}, J=7.5 \mathrm{~Hz}) ;{ }^{13} \mathrm{C} \mathrm{NMR}\left(100 \mathrm{MHz}, \mathrm{CDCl}_{3}\right) \delta 143.8,134.9,131.0,129.5,129.4$, 128.0, $59.3(\mathrm{~d}, J=2.5 \mathrm{~Hz}), 46.6(2 \mathrm{~d}, J=13.5,13.5 \mathrm{~Hz}), 31.4(\mathrm{~d}, J=5.5,5.5 \mathrm{~Hz}), 25.3,21.9,21.7,13.7$; ${ }^{31} \mathrm{P}$ NMR (121 MHz, $\left.\mathrm{CDCl}_{3}\right) \delta$ 19.49; MS (EI) m/e (rel intensity) 385 (8), 304 (14), 230 (88), 149 (19), 148 (27), 133 (100), 57 (24); HRMS (EI) m/e calcd $\left(\mathrm{M}^{+}\right)$385.1589, found 385.1589.

$N$-(1,3-Dimethyl-2-oxo-2 $\lambda^{5}$-[1,3,2] diazaphospholidin-2-yl)-4-methyl- $N$-(1-phenyl-allyl)benzenesulfonamide $(\mathbf{1 6 m})$.

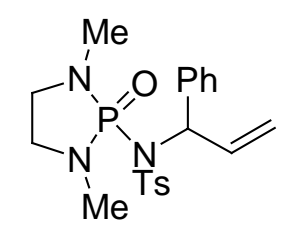

Flash chromatography (EtOAc:hexanes; 2:1) yielded a clear oil (75\%). $\mathrm{R}_{f}=0.29$ (EtOAc:hexanes; 3:1); ${ }^{1} \mathrm{H}$ NMR $\left(300 \mathrm{MHz}, \mathrm{CDCl}_{3}\right) \delta$ 7.58-7.50 (3H, m), 7.18-7.32 (6H, m), $6.86(1 \mathrm{H}, \mathrm{ddd}, J=17.5,10.5,9.0$ $\mathrm{Hz}), 5.92-5.78(1 \mathrm{H}, \mathrm{m}), 5.21(1 \mathrm{H}, \mathrm{d}, J=10.5 \mathrm{~Hz}), 5.10(1 \mathrm{H}, \mathrm{d}, J=17.5 \mathrm{~Hz}), 3.46-3.00(4 \mathrm{H}, \mathrm{m}), 2.40$ $(3 \mathrm{H}, \mathrm{s}), 2.24(3 \mathrm{H}, \mathrm{d}, J=10.5 \mathrm{~Hz}), 2.17(3 \mathrm{H}, \mathrm{d}, J=10.5 \mathrm{~Hz}) ;{ }^{13} \mathrm{C} \mathrm{NMR}\left(100 \mathrm{MHz}, \mathrm{CDCl}_{3}\right) \delta 143.7$, 141.0, 138.8, 137.6, 129.3, 129.2, 128.1, 127.9, 127.6, 119.1, 65.8, 46.6 (2d, J = 14.0, 14.0 Hz), 31.7 $(2 \mathrm{~d}, J=5.5,5.5 \mathrm{~Hz}), 21.7 ;{ }^{31} \mathrm{P} \mathrm{NMR}\left(121 \mathrm{MHz}, \mathrm{CDCl}_{3}\right) \delta 19.42$; MS (EI) $m / e$ (rel intensity) 419 (6), 322 (8), 265 (20), 264 (100), 149 (16), 133 (46), 117 (17), 91 (15); HRMS (EI) m/e calcd (M+) 419.1433, found 419.1439 . 
(E)- $N$-(1,3-Dimethyl-2-oxo-2 $\lambda^{5}$-[1,3,2] diazaphospholidin-2-yl)-4-methyl- $N$-(1-phenyl-but-2-enyl)benzenesulfonamide (16n).

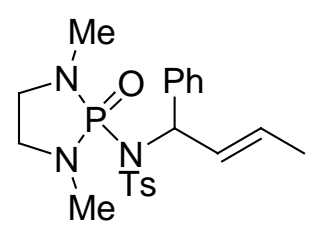

Flash chromatography (EtOAc:hexanes; 2:1) yielded a clear oil (76\%). $\mathrm{R}_{f}=0.30$ (EtOAc:hexanes; 4:1); IR (Thin Film) v 2935, 1495, 1340, 1270, 1164, 1040, 953, $668 \mathrm{~cm}^{-1} ;{ }^{1} \mathrm{H}$ NMR (300 MHz, CDCl 3$)$

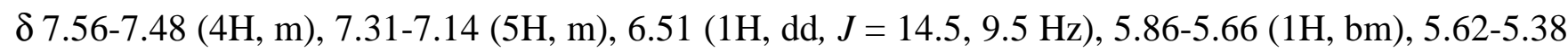
(1H, bm), 3.44-3.02 (4H, m), $2.40(3 \mathrm{H}, \mathrm{s}), 2.28$ (3H, bd, $J=10.0 \mathrm{~Hz}), 2.21(3 \mathrm{H}, \mathrm{dd}, J=10.5 \mathrm{~Hz}), 1.66$ $(3 \mathrm{H}, \mathrm{bd}, J=6.0 \mathrm{~Hz}) ;{ }^{13} \mathrm{C} \mathrm{NMR}\left(100 \mathrm{MHz}, \mathrm{CDCl}_{3}\right) \delta 143.7,141.7,130.7,130.1,129.1,128.9,128.0$, 128.0, 127.4, 65.4, $46.6(2 \mathrm{~d}, J=14.5,14.5 \mathrm{~Hz}), 31.2(2 \mathrm{~d}, J=15.3,15.3 \mathrm{~Hz}), 21.7,17.9$ (one aromatic C not observed); ${ }^{31} \mathrm{P}$ NMR (121 MHz, $\mathrm{CDCl}_{3}$ ) $\delta$ 19.50; MS (EI) m/e (rel intensity) 433 (5), 304 (12), 278 (100), 188 (35), 149 (37), 133 (88), 115 (33), 91 (53); HRMS (EI) m/e calcd (M+) 433.1589, found 433.1594.

(E)- $N$-(1,3-Dimethyl-2-oxo-2 $\lambda^{5}$-[1,3,2] diazaphospholidin-2-yl)-4-methyl- $N$-(1-methyl-3-phenylallyl)-benzenesulfonamide (160).

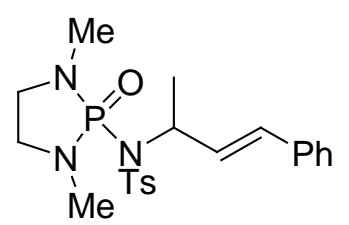

Flash chromatography (EtOAc:hexanes; 2:1) yielded a clear oil (80\%). $\mathrm{R}_{f}=0.43$ (EtOAc:hexanes; 3:1); IR (Thin Film) v 2934, 1340, 1165, 1040, 972, $914 \mathrm{~cm}^{-1} ;{ }^{1} \mathrm{H}$ NMR $\left(300 \mathrm{MHz}, \mathrm{CDCl}_{3}\right) \delta 7.89(2 \mathrm{H}, \mathrm{d}, J=$ 8.0 Hz), 7.32-7.20 (7H, m), $6.67(1 \mathrm{H}, \mathrm{dd}, J=16.0,8.0 \mathrm{~Hz}), 5.97(1 \mathrm{H}, \mathrm{d}, J=16.0 \mathrm{~Hz}), 4.57(1 \mathrm{H}, \mathrm{ddq}, J$ $=14.0,8.0,7.0 \mathrm{~Hz}), 3.42-3.14(4 \mathrm{H}, \mathrm{m}), 2.57(3 \mathrm{H}, \mathrm{d}, J=10.5 \mathrm{~Hz}), 2.43(3 \mathrm{H}, \mathrm{s}), 2.40(3 \mathrm{H}, \mathrm{d}, J=10.5$ $\mathrm{Hz}), 1.57(3 \mathrm{H}, \mathrm{d}, J=7.0 \mathrm{~Hz}) ;{ }^{13} \mathrm{C} \mathrm{NMR}\left(100 \mathrm{MHz}, \mathrm{CDCl}_{3}\right) \delta 144.0,138.3,136.4,131.8,131.6,129.7$, 128.6, 128.1, 127.7, 126.7, $59.0(\mathrm{~d}, J=2.5 \mathrm{~Hz}), 46.5$ (d, $J=5.5,5.5 \mathrm{~Hz}), 31.3$ (d, $J=5.5,5.5 \mathrm{~Hz}), 21.7$, 21.7; ${ }^{31} \mathrm{P}$ NMR (121 MHz, $\mathrm{CDCl}_{3}$ ) $\delta 19.59$; MS (EI) m/e (rel intensity) 433 (4), 278 (100), 149 (12), 133 (71), 91 (22); HRMS (EI) m/e calcd (M+) 433.1589, found 433.1603.

$(E)-(R)-N-\left(1,3-D i m e t h y l-2-o x o-2 \lambda^{5}\right.$-[1,3,2]diazaphospholidin-2-yl)- $N$-(1-ethyl-but-2-enyl)-4methyl-benzenesulfonamide (16q). 


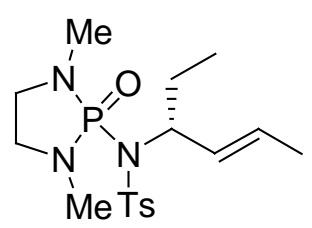

Flash chromatography (EtOAc:hexanes; 2:1) yielded a clear oil $(90 \%)$ with $91 \%$ ee as determined by chiral HPLC (Chiralcel AD column, 90:10; Hexanes:iPrOH at 1mL/min, $R$ isomer $=9.0 \mathrm{~min}, S$ isomer $=$ 10.0min); $[\alpha]_{\mathrm{D}}=+22.5^{\circ}\left(\mathrm{CHCl}_{3}, \mathrm{c}=0.95\right) ; \mathrm{R}_{f}=0.37$ (EtOAc:hexanes; 2:1); IR (Thin Film) v 2933, 1342, 1233, 1164, 1041, 950, 743, $668 \mathrm{~cm}^{-1} ;{ }^{1} \mathrm{H}$ NMR $\left(300 \mathrm{MHz}, \mathrm{CDCl}_{3}\right) \delta 7.75(2 \mathrm{H}, \mathrm{d}, J=8.5 \mathrm{~Hz})$, $7.29(2 \mathrm{H}, \mathrm{d}, J=8.0 \mathrm{~Hz}), 5.89$ (1H, ddd, $J=15.5,9.0,1.5 \mathrm{~Hz}), 5.21$ (1H, bm), 4.02 (1H, bm), 3.46-3.30 $(2 \mathrm{H}, \mathrm{m}), 3.22-3.12(2 \mathrm{H}, \mathrm{m}), 2.54(3 \mathrm{H}, \mathrm{bd}, J=10.5 \mathrm{~Hz}), 2.43(3 \mathrm{H}, \mathrm{s}), 2.42(3 \mathrm{H}, \mathrm{d}, J=10.5 \mathrm{~Hz}), 2.18$ $2.04(1 \mathrm{H}, \mathrm{m}), 1.70-1.58(1 \mathrm{H}, \mathrm{m}), 1.55(3 \mathrm{H}, 2, J=6.5 \mathrm{~Hz}), 0.75(3 \mathrm{H}, \mathrm{t}, J=7.5 \mathrm{~Hz}) ;{ }^{13} \mathrm{C} \mathrm{NMR}(100 \mathrm{MHz}$, $\left.\mathrm{CDCl}_{3}\right) \delta 143.7,134.9,131.5,131.0,129.3,128.0,65.7,46.6(2 \mathrm{~d}, J=5.5 \mathrm{~Hz}), 25.3,21.7,17.7,13.6$, 11.9; ${ }^{31} \mathrm{P}$ NMR (121 MHz, $\left.\mathrm{CDCl}_{3}\right) \delta$ 19.53; MS (EI) m/e (rel intensity) 385 (7), 356 (12), 292 (20), 230 (96), 148 (26), 133 (100), 91 (17); HRMS (EI) m/e calcd ( $\mathrm{M}^{+}$) 385.1589, found 385.1584.

\section{$(E)-(S)-N$-(1,3-Dimethyl-2-oxo-2 $\lambda^{5}$-[1,3,2] diazaphospholidin-2-yl)- $N$-(1-ethyl-but-2-enyl)-4-methyl-} benzenesulfonamide (16r).

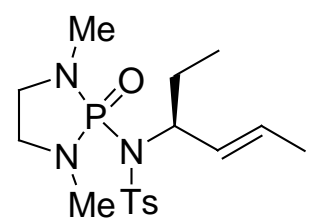

Flash chromatography (EtOAc:hexanes; $2: 1)$ yielded a clear oil $(88 \%)$ with $78 \%$ ee as determined by chiral HPLC (Chiralcel AD column, 90:10; Hexanes:iPrOH at $1 \mathrm{~mL} / \mathrm{min}, R$ isomer $=9.0 \mathrm{~min}, S$ isomer $=$ $10.0 \min ) ;[\alpha]_{\mathrm{D}}=-16.4^{\mathrm{o}}\left(\mathrm{CHCl}_{3}, \mathrm{c}=1.0\right) ;$

Absolute Stereochemistry Assignment: A sample of $\mathbf{1 6 q}$ was oxidatively cleaved to the methyl ester by ozonolysis in methanolic $\mathrm{NaOH}$ as described by Marshall and Garofalo ${ }^{1}$ ( $75 \%$ yield). The optical rotation of the resultant Methyl 2(R)-[N-(4-tolylsulfonyl)amino]butanoate $\left([\alpha]_{\mathrm{D}}=+17.9^{\circ}(\mathrm{EtOH}\right.$, $\mathrm{c}=2.1))$ was compared to rotations previously reported for the $S$ isomer $\left([\alpha]_{\mathrm{D}}=-22^{\mathrm{o}}(\mathrm{EtOH}, \mathrm{c}=1.0)\right)^{2}$. A sample of $\mathbf{1 8 q}$ was then hydrolyzed, tosylated under standard conditions and analyzed by chiral HPLC to correlate the HPLC retention times with the now known tosylated isomer.

\footnotetext{
${ }^{1}$ Marshall, J.A.; Garofalo, A.W. J. Org. Chem. 1993, 58, 3675-3680.

${ }^{2}$ Duréault, A.; Tranchepain, I.; Depezay, J-C. J. Org. Chem. 1989, 54, 5324-5330.
} 

benzenesulfonamide $(\mathbf{1 7} \mathrm{m})$.

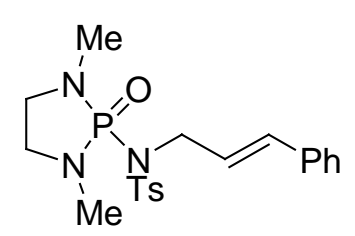

Flash Chromatography (EtOAc:hexanes; 2:1) yielded a white solid (75\%). $\mathrm{R}_{f}=0.38$ (EtOAc:hexanes; 3:1); ${ }^{1} \mathrm{H}$ NMR $\left(300 \mathrm{MHz}, \mathrm{CDCl}_{3}\right) \delta 7.77(2 \mathrm{H}, \mathrm{d}, J=8.0 \mathrm{~Hz}), 7.34-7.20(7 \mathrm{H}, \mathrm{m}), 6.55(1 \mathrm{H}, \mathrm{d}, J=16.0$ Hz), 6.24 (1H, dt, $J=16.0,7.0 \mathrm{~Hz}), 4.34$ (2H, ddd, $J=10.5,7.0,1.0 \mathrm{~Hz}), 3.40-3.26$ (2H, m), 3.24-3.12 (2H, m), $2.42(6 \mathrm{H}, \mathrm{d}, J=10.5 \mathrm{~Hz}), 2.40(3 \mathrm{H}, \mathrm{s}) ;{ }^{13} \mathrm{C} \mathrm{NMR}\left(100 \mathrm{MHz}, \mathrm{CDCl}_{3}\right) \delta 144.0,138.4,136.9$, 134.2, 129.6, 128.7, 127.9, 127.8, 126.8, 126.4, 51.4 (d, $J=2.5 \mathrm{~Hz}), 47.0(\mathrm{~d}, J=14.5 \mathrm{~Hz}), 31.4(\mathrm{~d}, J=$ $5.5 \mathrm{~Hz}$ ), 21.7; ${ }^{31} \mathrm{P}$ NMR (121 MHz, $\mathrm{CDCl}_{3}$ ) $\delta$ 18.89; MS (EI) m/e (rel intensity) 420 (1), 266 (23), 265 (100), 133 (88), 117 (13); HRMS (EI) m/e calcd $\left(\mathrm{M}^{+}\right)$420.1511, found 420.1518.

Rearrangement of diphenylphosphino iminodiazaphospholidines. In a flame dried round bottom flask under $\mathrm{N}_{2}$, a solution of iminodiazaphospholidine $(0.6 \mathrm{mmol})$ in $\mathrm{CH}_{2} \mathrm{Cl}_{2}(6 \mathrm{~mL})$ was added to $8 \mathrm{mg}$ of bis(acetonitrile)dichloropalladium(II) (5 mol\%) and freshly activated $4 \AA$ sieves (50 mg). The reaction was stirred for 16 hours under an atmosphere of $\mathrm{N}_{2}$ then checked for completion by ${ }^{31} \mathrm{P} N M R$. The reaction was then concentrated in vacuo, dissolved in a minimum amount of $\mathrm{CH}_{2} \mathrm{Cl}_{2}$, and purified by column chromatography.

(1,3-Dimethyl-2-oxo-2 $\lambda^{5}$-[1,3,2]diazaphospholidin-2-yl)-(1-ethyl-allyl)-phosphoramidic

acid diphenyl ester (18c).

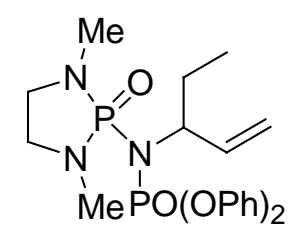

Flash chromatography (EtOAc:hexanes; 2:1) yielded a clear oil (from 12c 90\%, from 12d 89\%) $\mathrm{R}_{f}=$ 0.31 (EtOAc:hexanes; 5:1); IR (Thin Film) v 2934, 1591, 1489, 1286, 1191, 1164, 933, 771, $690 \mathrm{~cm}^{-1}$; ${ }^{1} \mathrm{H}$ NMR $\left(300 \mathrm{MHz}, \mathrm{CDCl}_{3}\right) \delta$ 7.37-7.12 (10H, m), $6.45(1 \mathrm{H}, \mathrm{ddd}, J=17.0,10.0,8.0 \mathrm{~Hz}), 5.23(1 \mathrm{H}, \mathrm{d}, J$ $=17.0 \mathrm{~Hz}), 5.21(1 \mathrm{H}, \mathrm{d}, J=10.0 \mathrm{~Hz}), 4.01(1 \mathrm{H}, \mathrm{m}), 3.10-3.40(4 \mathrm{H}, \mathrm{m}), 2.51(3 \mathrm{H}, \mathrm{d}, J=10.0 \mathrm{~Hz}), 2.44$ $(3 \mathrm{H}, \mathrm{d}, J=10.0 \mathrm{~Hz}), 2.40-2.22(1 \mathrm{H}, \mathrm{m}), 1.90-1.74(1 \mathrm{H}, \mathrm{m}), 0.90(3 \mathrm{H}, \mathrm{t}, J=7.5 \mathrm{~Hz}) ;{ }^{13} \mathrm{C} \mathrm{NMR}(100$ $\left.\mathrm{MHz}, \mathrm{CDCl}_{3}\right) \delta 151.1(\mathrm{~d}, J=7.0 \mathrm{~Hz}), 139.4,129.3(\mathrm{~d}, J=8.5 \mathrm{~Hz}), 125.2(\mathrm{~d}, J=5.5 \mathrm{~Hz}), 120.7(\mathrm{~d}, J=$ $4.5 \mathrm{~Hz}), 120.5(\mathrm{~d}, J=5.5 \mathrm{~Hz}), 118.2,65.4,46.8(\mathrm{~d}, J=11.5,11.5 \mathrm{~Hz}), 31.1(\mathrm{~d}, J=6.0,6.0 \mathrm{~Hz}), 28.4$, 
12.1; ${ }^{31} \mathrm{P}$ NMR $\left(121 \mathrm{MHz}, \mathrm{CDCl}_{3}\right) \delta 20.94(\mathrm{~d}, J=20.5 \mathrm{~Hz}),-4.44(\mathrm{~d}, J=21.5 \mathrm{~Hz})$; MS (EI) $m / e($ rel intensity) 449 (8), 421 (37), 392 (62), 380 (98), 356 (53), 325 (100), 296 (23), 288 (30), 133 (82), 85 (43), 77 (30); HRMS (EI) m/e calcd $\left(\mathrm{M}^{+}\right)$449.1633, found 449.1642.

(1,3-Dimethyl-2-oxo-2 $\lambda^{5}$-[1,3,2]diazaphospholidin-2-yl)-(2-methyl-allyl)-phosphoramidic acid diphenyl ester (18i).

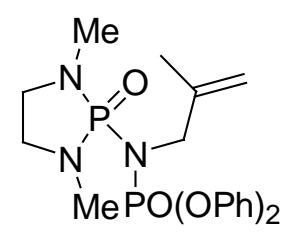

Flash chromatography (EtOAc:hexanes; 2:1) yielded a clear oil (91\%). $\mathrm{R}_{f}=0.27$ (EtOAc:hexanes; 5:1); IR (Thin Film) v 2928, 1591, 1489, 1278, 1192, 938, 873, 762, $\left.690 \mathrm{~cm}^{-1} ;{ }^{1} \mathrm{H} \mathrm{NMR} \mathrm{(300} \mathrm{MHz,} \mathrm{CDCl}_{3}\right)$ $\delta$ 7.34-7.12 (10H, m), $5.05(1 \mathrm{H}, \mathrm{s}), 4.93(1 \mathrm{H}, \mathrm{s}), 4.14(2 \mathrm{H}, \mathrm{dd}, J=13.5,13.5 \mathrm{~Hz}), 3.36-3.10(4 \mathrm{H}, \mathrm{m})$, $2.51(6 \mathrm{H}, \mathrm{dd}, J=10.0 \mathrm{~Hz}), 1.84(3 \mathrm{H}, \mathrm{s}) ;{ }^{13} \mathrm{C} \mathrm{NMR}\left(100 \mathrm{MHz}, \mathrm{CDCl}_{3}\right) \delta 151.0(\mathrm{~d}, J=7.5 \mathrm{~Hz}), 142.4$, 129.7, 125.1, 120.5 (d, $J=5.5 \mathrm{~Hz}), 112.3,53.5,46.8$ (d, $J=14.5 \mathrm{~Hz}), 31.4$ (d, $J=2.0 \mathrm{~Hz}), 20.7$ (d, $J=$ $2.5 \mathrm{~Hz}) ;{ }^{31} \mathrm{P}$ NMR $\left(121 \mathrm{MHz}, \mathrm{CDCl}_{3}\right) \delta 20.68(\mathrm{~d}, J=19.5 \mathrm{~Hz}),-3.67$ (d, $\left.J=19.5 \mathrm{~Hz}\right)$; MS (EI) $m / e$ (rel intensity) 435 (7), 407 (92), 380 (100), 342 (38), 323 (45), 302 (12), 174 (21), 133 (31), 77 (15); HRMS (EI) $m / e$ calcd $\left(\mathrm{M}^{+}\right) 435.1477$, found 435.1467 .

(E)-But-2-enyl-(1,3-dimethyl-2-oxo-2 $\lambda^{5}$-[1,3,2]diazaphospholidin-2-yl)-phosphoramidic

acid diphenyl ester (18j).

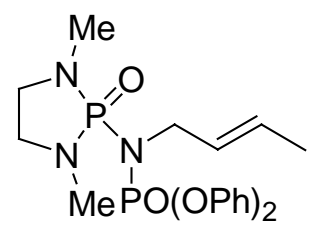

Flash chromatography (EtOAc:hexanes; 2:1) yielded a clear oil (86\%). $\mathrm{R}_{f}=0.28$ (EtOAc:hexanes; 5:1); IR (Thin Film) v 2934, 1591, 1489, 1286, 1191, 1164, 933, 771, $\left.690 \mathrm{~cm}^{-1} ;{ }^{1} \mathrm{H} \mathrm{NMR} \mathrm{(300} \mathrm{MHz,} \mathrm{CDCl} 3\right)$ $\delta$ 7.36-7.04 (10H, m), $5.81(1 \mathrm{H}, \mathrm{dt}, J=15.5,6.0 \mathrm{~Hz}), 5.71(1 \mathrm{H}, \mathrm{dq}, J=15.5,6.5 \mathrm{~Hz}), 4.09(2 \mathrm{H}, \mathrm{ddd}, J=$ 14.0, 14.0, $6.0 \mathrm{~Hz}), 3.30-3.08(4 \mathrm{H}, \mathrm{m}), 2.40(6 \mathrm{H}, \mathrm{d}, J=10.0 \mathrm{~Hz}), 1.69(3 \mathrm{H}, \mathrm{d}, J=6.0 \mathrm{~Hz}) ;{ }^{13} \mathrm{C}$ NMR $\left(100 \mathrm{MHz} \mathrm{CDCl}_{3}\right) \delta 150.8(\mathrm{~d}, J=8.0 \mathrm{~Hz}), 129.8,129.7,128.5,125.2,120.6(\mathrm{~d}, J=5.5 \mathrm{~Hz}), 50.0,46.6$ $(\mathrm{d}, J=14.5 \mathrm{~Hz}), 36.9(\mathrm{~d}, J=5.5,5.5 \mathrm{~Hz}), 17.8 ;{ }^{31} \mathrm{P} \mathrm{NMR}\left(121 \mathrm{MHz}, \mathrm{CDCl}_{3}\right) \delta 20.43(\mathrm{~d}, J=20.0 \mathrm{~Hz}),-$ 4.02 (d, $J=20.0 \mathrm{~Hz}$ ); MS (EI) m/e (rel intensity) 435 (11), 410 (19), 393 (97), 380 (100), 342 (32), 325 
(65), 323 (76), 302 (29), 294 (27), 288 (27), 133 (26); HRMS (EI) m/e calcd $\left(\mathrm{M}^{+}\right.$) 435.1477, found 435.1472 .

\section{(E)-(1,3-Dimethyl-2-oxo-2 $\lambda^{5}$-[1,3,2]diazaphospholidin-2-yl)-(1-methyl-pent-2-enyl)-} phosphoramidic acid diphenyl ester (181).

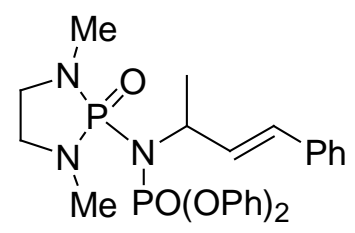

Flash chromatography (EtOAc:hexanes; 2:1) yielded a clear oil (93\%). $\mathrm{R}_{f}=0.25$ (EtOAc:hexanes; 5:1);

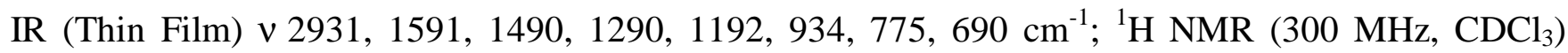
$\delta$ 7.36-7.10 (10H, m), $6.11(1 \mathrm{H}, \mathrm{dd}, J=15.5,8.0 \mathrm{~Hz}), 5.67(1 \mathrm{H}, \mathrm{dt}, J=15.0,6.5 \mathrm{~Hz}), 4.40(1 \mathrm{H}, \mathrm{m})$, 3.38-3.08 (4H, m), $2.50(3 \mathrm{H}, \mathrm{d}, J=10.0 \mathrm{~Hz}), 2.43(3 \mathrm{H}, \mathrm{d}, J=10.0 \mathrm{~Hz}), 2.04(2 \mathrm{H}, \mathrm{qd}, J=7.5,6.5 \mathrm{~Hz})$, $1.61(3 \mathrm{H}, \mathrm{d}, J=7.0 \mathrm{~Hz}), 0.96(3 \mathrm{H}, \mathrm{t}, J=7.5 \mathrm{~Hz}) ;{ }^{13} \mathrm{C} \mathrm{NMR}\left(100 \mathrm{MHz}, \mathrm{CDCl}_{3}\right) \delta 151.1(\mathrm{~d}, J=7.5 \mathrm{~Hz})$, 134.4, 131.9, 129.7 (d, $J=10.0 \mathrm{~Hz}), 125.1(\mathrm{~d}, J=8.5 \mathrm{~Hz}), 120.6$ (d, $J=5.5 \mathrm{~Hz}), 58.2,46.9(\mathrm{~d}, J=4.5$ $\mathrm{Hz}), 31.1(\mathrm{~d}, J=6.0 \mathrm{~Hz}), 25.4,22.2,13.7 ;{ }^{31} \mathrm{P}$ NMR $\left(121 \mathrm{MHz}, \mathrm{CDCl}_{3}\right) \delta 20.89(\mathrm{~d}, J=21.5 \mathrm{~Hz}),-4.36$ (d, $J=21.5 \mathrm{~Hz}$ ); MS (EI) m/e (rel intensity) 463 (10), 382 (46), 381 (56), 380 (79), 330 (33), 325 (100), 288 (41), 433 (55), 85 (37), 55 (33); HRMS (EI) m/e calcd $\left(\mathrm{M}^{+}\right)$463.1789, found 463.1788.

\section{$(E)-(R)-\left(1,3-D i m e t h y l-2-o x 0-2 \lambda^{5}-[1,3,2]\right.$ diazaphospholidin-2-yl)-(1-ethyl-but-2-enyl)-}

phosphoramidic acid diphenyl ester (18q).

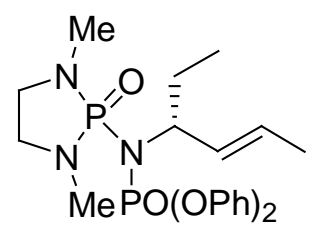

Flash chromatography (EtOAc:hexanes; 2:1) yielded a clear oil $(84 \%)$ with $91 \%$ ee as determined by chiral HPLC (Chiralcel AD column, 85:15; Hexanes:iPrOH at 1mL/min, $R$ isomer $=7.61 \mathrm{~min}, S$ isomer $=$ 9.19min); $[\alpha]_{\mathrm{D}}=+10.9^{\circ}\left(\mathrm{CHCl}_{3}, \mathrm{c}=1.0\right) ; \mathrm{R}_{f}=0.30$ (EtOAc:hexanes; 3:1); IR (Thin Film) v 2932, 1447, 1357, 1271, 1163, 1043, 990, $672 \mathrm{~cm}^{-1} ;{ }^{1} \mathrm{H}$ NMR (300 MHz, CDCl 3$) \delta 7.38-7.10(10 \mathrm{H}, \mathrm{m}), 6.08(1 \mathrm{H}$, $\mathrm{dd}, J=15.0,9.0 \mathrm{~Hz}), 5.68(1 \mathrm{H}, \mathrm{dq}, J=15.0,6.5 \mathrm{~Hz}), 4.10-3.86(1 \mathrm{H}, \mathrm{m}), 3.38-3.10(4 \mathrm{H}, \mathrm{m}), 2.50(3 \mathrm{H}, \mathrm{d}$, $J=10.0 \mathrm{~Hz}), 2.43(3 \mathrm{H}, \mathrm{d}, J=10.0 \mathrm{~Hz}), 2.32-2.20(1 \mathrm{H}, \mathrm{m}), 1.90-1.75(1 \mathrm{H}, \mathrm{m}), 1.71(3 \mathrm{H}, \mathrm{d}, J=6.5 \mathrm{~Hz})$, $0.88(3 \mathrm{H}, \mathrm{t}, J=7.5 \mathrm{~Hz}) ;{ }^{13} \mathrm{C}$ NMR $\left(100 \mathrm{MHz}, \mathrm{CDCl}_{3}\right) \delta 151.1(\mathrm{~d}, J=7.0 \mathrm{~Hz}), 132.2,129.7(\mathrm{~d}, J=11.5$ $\mathrm{Hz}), 129.4,125.1$ (d, $J=10.0 \mathrm{~Hz}), 120.5$ (d, $J=5.0 \mathrm{~Hz}), 64.9,46.9(2 \mathrm{~d}, J=14.5 \mathrm{~Hz}), 31.1(2 \mathrm{~d}, J=6.0$ $\mathrm{Hz}), 28.6,17.8,12.2 ;{ }^{31} \mathrm{P} \mathrm{NMR}\left(121 \mathrm{MHz}, \mathrm{CDCl}_{3}\right) \delta 21.00(\mathrm{~d}, J=21.5 \mathrm{~Hz}),-4.39(\mathrm{~d}, J=21.5 \mathrm{~Hz}) ; \mathrm{MS}$ 
(EI) m/e (rel intensity) 463 (16), 434 (21), 421 (33), 392 (23), 380 (100), 325 (96), 296 (18), 288 (34), 133 (56), 85 (33), HRMS (EI) m/e calcd $\left(\mathrm{M}^{+}\right)$463.1789, found 463.1785.

\section{(E)-(S)-(1,3-Dimethyl-2-oxo-2 $\lambda^{5}$-[1,3,2] diazaphospholidin-2-yl)-(1-ethyl-but-2-enyl)-} phosphoramidic acid diphenyl ester (18r).

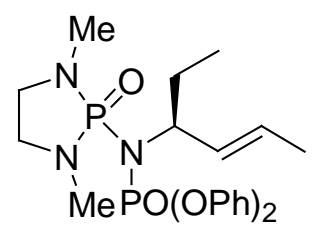

Flash chromatography (EtOAc:hexanes; 2:1) yielded a clear oil (83\%) with $70 \%$ ee as determined by chiral HPLC (Chiralcel AD column, 85:15;Hexanes:iPrOH at $1 \mathrm{~mL} / \mathrm{min}, R$ isomer $=7.61 \mathrm{~min}, S$ isomer $=$ $9.19 \min ) ;[\alpha]_{\mathrm{D}}=-7.7^{\circ}\left(\mathrm{CHCl}_{3}, \mathrm{c}=1.0\right)$

Hydrolysis of tosyl phosphoramides. To a solution of rearranged phospholidine ( $0.5 \mathrm{mmol})$ in THF (5 $\mathrm{mL})$, an aqueous $1 \mathrm{M} \mathrm{HCl}$ solution $(5 \mathrm{~mL})$ was added. The reaction was stirred overnight and checked for completion by TLC. The reaction was then quenched by the addition of sat. $\mathrm{NaHCO}_{3}(20 \mathrm{~mL})$. The THF was removed in vacuo and the resultant solution extracted with $\mathrm{CH}_{2} \mathrm{Cl}_{2}(3 \times 15 \mathrm{~mL})$. The combined organic layers were dried over $\mathrm{MgSO}_{4}$, filtered, concentrated, and purified by flash chromatography.

\section{$N$-(1-Propyl-allyl)-4-methyl-benzenesulfonamide (19a).}

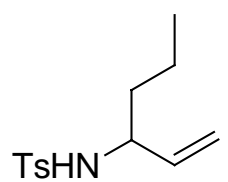

(Chao, B.; Dittmer, D. C. Tetrahedron Lett. 2001, 42, 5789-5792). Flash chromatography (EtOAc:hexanes; 1:3) yielded a clear oil (90\% and $86 \%)$; ee determined by chiral HPLC (15cm Chiralpak AD column with a 5cm Chiralcel OD guard, 96:4; Hexanes:iPrOH at $1 \mathrm{~mL} / \mathrm{min}, R$ isomer $=$ $12.7 \mathrm{~min}, S$ isomer $=14.9 \mathrm{~min}) ; R$ isomer $(94 \%$ ee $)[\alpha]_{\mathrm{D}}=-5.1^{\circ}\left(\mathrm{CHCl}_{3}, \mathrm{c}=1.0\right)$ Lit. for $S$ isomer: $[\alpha]_{\mathrm{D}}=-$ $3.5^{\mathrm{o}}\left(\mathrm{CHCl}_{3}, \mathrm{c}=2.0\right) ; \mathrm{R}_{f}=0.50\left(\right.$ EtOAc:hexanes; 1:4); ${ }^{1} \mathrm{H}$ NMR $\left(300 \mathrm{MHz}, \mathrm{CDCl}_{3}\right) \delta 7.80(2 \mathrm{H}, \mathrm{d}, J=$ $8.0 \mathrm{~Hz}), 7.33(2 \mathrm{H}, \mathrm{d}, J=8.0 \mathrm{~Hz}), 5.59(1 \mathrm{H}, \mathrm{ddd}, J=17.0,10.0,6.5 \mathrm{~Hz}), 5.03(1 \mathrm{H}, \mathrm{d}, J=17.0 \mathrm{~Hz}), 5.00$ $(1 \mathrm{H}, \mathrm{d}, J=10.0 \mathrm{~Hz}), 4.71(1 \mathrm{H}, \mathrm{d}, J=8.0 \mathrm{~Hz}), 3.81(1 \mathrm{H}, \mathrm{m}), 2.48(3 \mathrm{H}, \mathrm{s}), 1.49(2 \mathrm{H}, \mathrm{dt}, J=8.0,7.5 \mathrm{~Hz})$, $1.32(2 \mathrm{H}, \mathrm{tq}, J=7.5,7.5 \mathrm{~Hz}), 0.89(3 \mathrm{H}, \mathrm{t}, J=7.0 \mathrm{~Hz}) ;{ }^{13} \mathrm{C} \mathrm{NMR}\left(100 \mathrm{MHz}, \mathrm{CDCl}_{3}\right) \delta 143.2,138.1$, 137.9, 129.5, 127.2, 115.8, 56.1, 37.7, 21.6, 18.5, 13.7. 


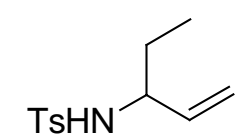

(Sisko, J.; Weinreb, S.M. J. Org. Chem., 1990, 55(1), 393). Flash chromatography (EtOAc:hexanes; 1:3) yielded a clear oil ( $88 \%$ and $88 \%$ ); ee determined by chiral HPLC (15cm Chiralpak AD column with a 5cm Chiralpak $\mathrm{AD}$ guard, 96:4;Hexanes:iPrOH at $1 \mathrm{~mL} / \mathrm{min}, S$ isomer $=23.5 \mathrm{~min}, R$ isomer $=$ 27.1min); $[\alpha]_{\mathrm{D}}=-7.2^{\mathrm{o}}: \mathrm{R}_{f}=0.42$ (EtOAc:hexanes; $\left.1: 1\right) ;{ }^{1} \mathrm{H} \mathrm{NMR}\left(300 \mathrm{MHz}, \mathrm{CDCl}_{3}\right) \delta 7.75(2 \mathrm{H}, \mathrm{d}, J=$ $8.0 \mathrm{~Hz}), 7.27(2 \mathrm{H}, \mathrm{d}, J=8.0 \mathrm{~Hz}), 5.54(1 \mathrm{H}, \mathrm{ddd}, J=17.0,10.5,0.5 \mathrm{~Hz}), 4.98(1 \mathrm{H}, \mathrm{d}, J=17.0 \mathrm{~Hz}), 4.96$ $(1 \mathrm{H}, \mathrm{d}, J=10.0 \mathrm{~Hz}), 4.77(1 \mathrm{H}, \mathrm{d}, J=8.0 \mathrm{~Hz}), 3.69(1 \mathrm{H}, \mathrm{dtd}, J=7.5,7.5,7.5 \mathrm{~Hz}), 2.42(3 \mathrm{H}, \mathrm{s}), 1.50$ $(2 \mathrm{H}, \mathrm{dq}, J=7.5,7.5 \mathrm{~Hz}), 0.82(3 \mathrm{H}, \mathrm{t}, J=7.5 \mathrm{~Hz}) ;{ }^{13} \mathrm{C} \mathrm{NMR}\left(100 \mathrm{MHz}, \mathrm{CDCl}_{3}\right) \delta 143.4,138.3,137.7$, $129.7,127.4,116.2,57.9,28.7,21.7,9.9$.

\section{$N$-(1-Methyl-allyl)-4-methyl-benzenesulfonamide (19g).}

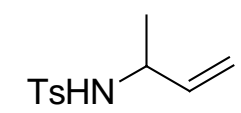

(Moriwake, T.; Hamano, S.; Saito, S.; Torii, S.; Kashino, S.; J. Org. Chem. 1989, 54, 4114-4120). Flash chromatography (EtOAc:hexanes; 1:3) yielded a clear oil (82\% and 80); ee determined by chiral HPLC (15cm Chiralpak AD column with a 5cm Chiralpak AD guard, 97:3;Hexanes:iPrOH at 1mL/min, $S$ isomer $=36.0 \mathrm{~min}, R$ isomer $=38.1 \mathrm{~min}) ; R$ Isomer $(91 \%$ ee $):[\alpha]_{\mathrm{D}}=+4.5^{\circ}\left(\mathrm{CHCl}_{3}, \mathrm{c}=0.5\right)$ Lit. for $S$ isomer: $[\alpha]_{\mathrm{D}}=+6.39^{\circ}\left(\mathrm{CHCl}_{3}, \mathrm{c}=2.69\right) ; \mathrm{R}_{f}=0.50$ (EtOAc:hexanes; 1:4); ${ }^{1} \mathrm{H}$ NMR $\left(300 \mathrm{MHz}, \mathrm{CDCl}_{3}\right)$ $\delta 7.77(2 \mathrm{H}, \mathrm{d}, J=8.0 \mathrm{~Hz}), 7.29(2 \mathrm{H}, \mathrm{d}, J=8.5 \mathrm{~Hz}), 5.64(1 \mathrm{H}, \mathrm{ddd}, J=16.5,10.5,6.0 \mathrm{~Hz}), 5.03(1 \mathrm{H}, \mathrm{d}$, $J=17.0 \mathrm{~Hz}), 4.95(1 \mathrm{H}, \mathrm{d}, J=10.0 \mathrm{~Hz}), 4.91(1 \mathrm{H}, \mathrm{d}, J=6.5 \mathrm{~Hz}), 3.88(1 \mathrm{H}, \mathrm{ddq}, J=7.0,7.0,7.0 \mathrm{~Hz})$, $2.42(3 \mathrm{H}, \mathrm{s}), 1.16(3 \mathrm{H}, \mathrm{d}, J=6.5 \mathrm{~Hz}) ;{ }^{13} \mathrm{C} \mathrm{NMR}\left(100 \mathrm{MHz}, \mathrm{CDCl}_{3}\right) \delta 143.2,139.0,138.0,129.6,127.1$, $115.0,51.6,21.5,21.5$.

\section{4-Methyl- $N$-(2-methyl-allyl)-benzenesulfonamide (19i).}

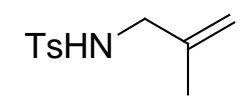

(Onistschenko, A.; Buchholz, B.; Stamm, H. Tetrahedron, 1987, 43(3), 565). Flash chromatography (EtOAc:hexanes; 1:3) yielded a clear oil (97\%): $\mathrm{R}_{f}=0.40$ (EtOAc:hexanes; 1:1); ${ }^{1} \mathrm{H}$ NMR $(300 \mathrm{MHz}$, $\left.\mathrm{CDCl}_{3}\right) \delta 7.76(2 \mathrm{H}, \mathrm{d}, J=8.5 \mathrm{~Hz}), 7.31(2 \mathrm{H}, \mathrm{d}, J=8.0 \mathrm{~Hz}), 4.86(1 \mathrm{H}, \mathrm{bs}), 4.82(1 \mathrm{H}, \mathrm{bs}), 4.63(1 \mathrm{H}, \mathrm{t}, J=$ 
$6.5 \mathrm{~Hz}), 3.48(2 \mathrm{H}, \mathrm{d}, J=6.5 \mathrm{~Hz}), 2.43(3 \mathrm{H}, \mathrm{s}), 1.68(3 \mathrm{H}, \mathrm{s}) ;{ }^{13} \mathrm{C} \mathrm{NMR}\left(100 \mathrm{MHz}, \mathrm{CDCl}_{3}\right) \delta 143.7$, $140.7,137.2,129.9,127.3,113.0,49.3,21.7,20.3$.

\section{(E)-N-But-2-enyl-4-methyl-benzenesulfonamide (19j).}

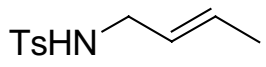

(McFarland, J.W.; Schut, D.; Zwaneburg, B. J. Org. Chem., 1981, 37(2), 389). ${ }^{3}$ Flash chromatography (EtOAc:hexanes; 1:3) yielded a clear oil (93\%): $\mathrm{R}_{f}=0.38$ (EtOAc:hexanes; 1:1); ${ }^{1} \mathrm{H}$ NMR $(300 \mathrm{MHz}$, $\left.\mathrm{CDCl}_{3}\right) \delta 7.75(2 \mathrm{H}, \mathrm{d}, J=8.0 \mathrm{~Hz}), 7.31(2 \mathrm{H}, \mathrm{d}, J=8.0 \mathrm{~Hz}), 5.56(1 \mathrm{H}, \mathrm{dqt}, J=15.5,6.5,1.5 \mathrm{~Hz}), 5.33$ $(1 \mathrm{H}, \mathrm{dtq}, J=15.5,6.5,1.5 \mathrm{~Hz}), 4.52(1 \mathrm{H}, \mathrm{t}, J=6.0 \mathrm{~Hz}), 3.50(2 \mathrm{H}, \mathrm{ddd}, J=6.5,6.0,1.5 \mathrm{~Hz}), 2.43(3 \mathrm{H}$, s), $1.60(3 \mathrm{H}, \mathrm{dd}, J=6.5,1.5 \mathrm{~Hz}) ;{ }^{13} \mathrm{C} \mathrm{NMR}\left(100 \mathrm{MHz}, \mathrm{CDCl}_{3}\right) \delta 143.6,138.7,129.5,128.4,126.8$, $123.5,40.3,20.0,11.7$.

$N$-Cyclohex-2-enyl-4-methyl-benzenesulfonamide (19k).

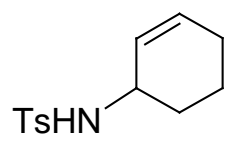

(McIntosh, M.C.; Weinreb, S.M. J. Org. Chem. 1993, 4823). Flash chromatography (EtOAc:hexanes; 1:3) yielded a clear oil oil (90\%): $\mathrm{R}_{f}=0.42$ (EtOAc:hexanes; 1:1); ${ }^{1} \mathrm{H}$ NMR $\left(300 \mathrm{MHz}, \mathrm{CDCl}_{3}\right) \delta 7.76$ $(2 \mathrm{H}, \mathrm{d}, J=8.5 \mathrm{~Hz}), 7.28(2 \mathrm{H}, \mathrm{d}, J=8.0 \mathrm{~Hz}), 5.83(1 \mathrm{H}, \mathrm{m}), 5.33(1 \mathrm{H}, \mathrm{m}), 4.41(1 \mathrm{H}, \mathrm{m}), 3.86(1 \mathrm{H}, \mathrm{m})$, $2.42(3 \mathrm{H}, \mathrm{s}), 2.02-1.85(2 \mathrm{H}, \mathrm{m}), 1.82-1.43(4 \mathrm{H}, \mathrm{m}) ;{ }^{13} \mathrm{C} \mathrm{NMR}\left(100 \mathrm{MHz}, \mathrm{CDCl}_{3}\right) \delta 143.2,138.3,131.4$, $129.6,127.1,127.0,49.0,30.2,24.4,21.5,19.3$.

(E)-4-Methyl- $N$-(1-methyl-pent-2-enyl)-benzenesulfonamide (19l).

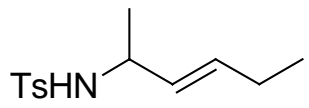

(Toda, A.; Aoyama, H.; Mimura, N.; Ohno, H.; Fujii, N.; Ibuka, T. J. Org. Chem. 1998, 63(20), 7053). Flash chromatography (EtOAc:hexanes; 1:3) yielded a clear oil (83\%): $\mathrm{R}_{f}=0.30$ (EtOAc:hexanes; 1:1); ${ }^{1} \mathrm{H}$ NMR $\left(300 \mathrm{MHz}, \mathrm{CDCl}_{3}\right) \delta 7.73(2 \mathrm{H}, \mathrm{d}, J=8.5 \mathrm{~Hz}), 7.27(2 \mathrm{H}, \mathrm{d}, J=8.0 \mathrm{~Hz}), 5.45(1 \mathrm{H}, \mathrm{dtd}, J=$ $15.5,6.5,1.0 \mathrm{~Hz}), 5.14(1 \mathrm{H}, \mathrm{ddt}, J=15.5,6.5,1.5 \mathrm{~Hz}), 4.27(1 \mathrm{H}, \mathrm{d}, J=7.5 \mathrm{~Hz}), 3.87(1 \mathrm{H}, \mathrm{dq}, J=7.0$, $6.5 \mathrm{~Hz}), 2.42(3 \mathrm{H}, \mathrm{s}), 1.87(2 \mathrm{H}, \mathrm{qd}, J=7.5,6.5 \mathrm{~Hz}), 1.16(3 \mathrm{H}, \mathrm{d}, J=6.5 \mathrm{~Hz}), 0.84(3 \mathrm{H}, \mathrm{t}, J=7.5 \mathrm{~Hz})$; ${ }^{13} \mathrm{C} \mathrm{NMR}\left(100 \mathrm{MHz}, \mathrm{CDCl}_{3}\right) \delta 143.6,138.7,129.5,128.4,126.8,123.5,40.3,24.3,20.0,18.7,11.7$.

\footnotetext{
${ }^{3}$ For (Z) literature data, see: Oppolzer, W.; Furstner, A. Helv. Chim. Acta. 76(6), 2329.
} 


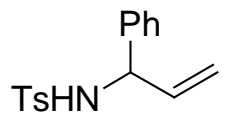

(Toshimitsu, A.; Kusumoto, T.O; Oida, T.; Tanimoto, S.; Bull. Chem. Soc. Jpn. 1991, 2148). Flash chromatography (EtOAc:hexanes; 1:3) yielded a white solid (85\%): $\mathrm{R}_{f}=0.42$ (EtOAc:hexanes; 1:1); ${ }^{1} \mathrm{H}$ NMR (300 MHz, $\left.\mathrm{CDCl}_{3}\right) \delta 7.66(2 \mathrm{H}, \mathrm{d}, J=8.5 \mathrm{~Hz}), 7.35-6.82(7 \mathrm{H}, \mathrm{m}), 5.86(1 \mathrm{H}, \mathrm{ddd}, J=16.5,11.0$, $6.5 \mathrm{~Hz}), 5.20-5.04(3 \mathrm{H}, \mathrm{m}), 4.93(1 \mathrm{H}, \mathrm{dd}, J=6.5,6.5 \mathrm{~Hz}), 2.43(3 \mathrm{H}, \mathrm{s}) ;{ }^{13} \mathrm{C} \mathrm{NMR}\left(100 \mathrm{MHz}, \mathrm{CDCl}_{3}\right)$ $\delta 144.5,137.1,135.1,130.8,129.7,128.8,127.6,127.3,123.4,116.6,59.8,21.4$.

(E)-4-Methyl- $N$-(1-phenyl-but-2-enyl)-benzenesulfonamide (19n).<smiles>C/C=C/C(N[GaH])c1ccccc1</smiles>

(Toshimitsu, A.; Kusumoto, T.O; Oida, T.; Tanimoto, S.; Bull. Chem. Soc. Jpn. 1991, 2148). Flash chromatography (EtOAc:hexanes; 1:3) yielded a white solid $(80 \%): \mathrm{R}_{f}=0.42($ EtOAc:hexanes; $1: 1) ;{ }^{1} \mathrm{H}$ $\operatorname{NMR}\left(300 \mathrm{MHz}, \mathrm{CDCl}_{3}\right) \delta 7.66(2 \mathrm{H}, \mathrm{d}, J=8.5 \mathrm{~Hz}), 7.35-6.82(7 \mathrm{H}, \mathrm{m}), 5.84(1 \mathrm{H}, \mathrm{dd}, J=16.0,6.5 \mathrm{~Hz})$, 5.16-5.04 (1H, m), $4.93(1 \mathrm{H}, \mathrm{dd}, J=6.5,6.5 \mathrm{~Hz}), 2.89(1 \mathrm{H}, \mathrm{bd}), 2.45(3 \mathrm{H}, \mathrm{s}), 1.71(3 \mathrm{H}, \mathrm{d}, J=7.0) ;{ }^{13} \mathrm{C}$ NMR (100 MHz, $\left.\mathrm{CDCl}_{3}\right) \delta 144.2,136.1,134.7,131.3,129.3,128.2,126.6,126.3,123.5,116.6,56.8$, 20.4, 17.2.

(E)-4-Methyl-N-(1-methyl-3-phenyl-allyl)-benzenesulfonamide (190).

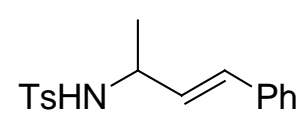

(Robin, S.; Rousseau, G. Eur. J. Org. Chem. 2000, (17), 3007; Bussas, R.; Kresze, G. Liebigs Annalen der Chemie, 1980, (4), 629). Flash chromatography (EtOAc:hexanes; 1:3) yielded a white solid (78\%): $\mathrm{R}_{f}=0.2$ (EtOAc:hexanes; 1:1); ${ }^{1} \mathrm{H} \mathrm{NMR}\left(300 \mathrm{MHz}, \mathrm{CDCl}_{3}\right) \delta 7.76(2 \mathrm{H}, \mathrm{d}, J=8.5 \mathrm{~Hz}), 7.28-7.10(7 \mathrm{H}$, m), $6.27(1 \mathrm{H}, \mathrm{d}, J=15.5 \mathrm{~Hz}), 5.83(1 \mathrm{H}, \mathrm{dd}, J=16.0,6.0 \mathrm{~Hz}), 5.04(1 \mathrm{H}, \mathrm{d}, J=7.5 \mathrm{~Hz}), 4.06(1 \mathrm{H}, \mathrm{dqd}, J$ $=7.5,6.5,6.0 \mathrm{~Hz}), 2.31(3 \mathrm{H}, \mathrm{s}), 1.25(3 \mathrm{H}, \mathrm{d}, J=6.5 \mathrm{~Hz}) ;{ }^{13} \mathrm{C} \mathrm{NMR}\left(100 \mathrm{MHz}, \mathrm{CDCl}_{3}\right) \delta 144.0,138.2$, $136.9,131.8,131.5,129.6,128.6,128.1,127.8,126.7,46.6,31.35,21.65$.

(E)-(R)-N-(1-Ethyl-but-2-enyl)-4-methyl-benzenesulfonamide (19q).

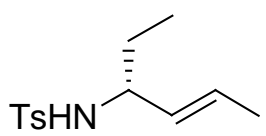


(Yu, X-Q.; Huang, J-S.; Zhou, X-G.; Che, C-M. Organic Letters 2000, (15), 2233). Flash chromatography (EtOAc:hexanes; 1:3) yielded a clear oil (82\%) with $90 \%$ ee as determined by chiral HPLC (Chiralpak AD column, 97:3; Hexanes:iPrOH at 1mL/min, $S$ isomer $=23.75 \mathrm{~min}, R$ isomer $=$ 25.41min); $[\alpha]_{\mathrm{D}}=+16.4^{\mathrm{o}}\left(\mathrm{CHCl}_{3}, \mathrm{c}=1.0\right) ; \mathrm{R}_{f}=0.35$ (EtOAc:hexanes; $\left.1: 1\right) ;{ }^{1} \mathrm{H} \mathrm{NMR}\left(500 \mathrm{MHz}, \mathrm{CDCl}_{3}\right)$ $\delta 7.72(2 \mathrm{H}, \mathrm{d}, J=8.5 \mathrm{~Hz}), 7.27(2 \mathrm{H}, \mathrm{d}, J=8.0 \mathrm{~Hz}), 5.34(1 \mathrm{H}, \mathrm{dq}, J=15.0,6.5 \mathrm{~Hz}), 5.05(1 \mathrm{H}, \mathrm{ddd}, J=$ 15.5, 7.5, $1.5 \mathrm{~Hz}), 4.60(1 \mathrm{H}, \mathrm{d}, J=7.0 \mathrm{~Hz}), 3.61(1 \mathrm{H}$, quint, $J=7.0 \mathrm{~Hz}), 2.42(3 \mathrm{H}, \mathrm{s}), 1.54-1.39(2 \mathrm{H}$, m), $1.48(3 \mathrm{H}, \mathrm{d}, J=6.5 \mathrm{~Hz}), 0.81(3 \mathrm{H}, \mathrm{t}, J=7.5 \mathrm{~Hz}) ;{ }^{13} \mathrm{C} \mathrm{NMR}\left(125 \mathrm{MHz}, \mathrm{CDCl}_{3}\right) \delta 143.1,138.5$, $130.5129 .5,127.7124 .4,57.7,29.1,21.7,17.7,10.1$.

(E)-(S)-N-(1-Ethyl-but-2-enyl)-4-methyl-benzenesulfonamide (19r).

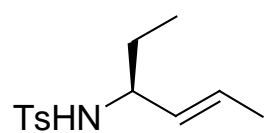

Flash chromatography (EtOAc:hexanes; 1:3) yielded a clear oil (90\%) with $70 \%$ ee as determined by chiral HPLC (Chiralcel AD column, 97:3;Hexanes:iPrOH at 1mL/min, $S$ isomer $=23.75 \mathrm{~min}, R$ isomer $=$ 25.41min $) ;[\alpha]_{\mathrm{D}}=-12.6\left(\mathrm{CHCl}_{3}, \mathrm{c}=1.0\right)$.

Hydrolysis of diphenylphosphino phosphoramides. To a solution of rearranged phospholidine $(0.5$ $\mathrm{mmol})$ in $\mathrm{MeOH}(5 \mathrm{~mL})$, an aqueous $1 \mathrm{M} \mathrm{HCl}$ solution $(5 \mathrm{~mL})$ was added. The reaction was stirred overnight and checked for completion by TLC. The reaction was then concentrated under high vacuum to yield a light residue. The residue was then purified by flash chromatography on a short silica column $(10-15 \mathrm{~cm})$.

\section{1-Ethyl-allylamine hydrochloride (20c).}

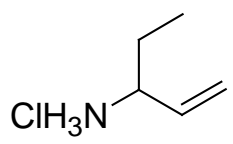

(Turk, S.D.; Louthan, R.P.; Cobb, R.L.; Bresson, C.R. J. Org. Chem. 1962, (15), 2846-2853). Flash Chromatography (Gradient of 5-15\% $\mathrm{MeOH}$ in $\mathrm{CH}_{2} \mathrm{Cl}_{2}$ ) yielded a white solid ( $81 \%$ and $81 \%$ ). $\mathrm{R}_{f}=0.14$ $\left(\mathrm{MeOH}: \mathrm{CH}_{2} \mathrm{Cl}_{2} ; 5: 95\right) ;{ }^{1} \mathrm{H} \mathrm{NMR}\left(300 \mathrm{MHz}, \mathrm{CD}_{3} \mathrm{OD}\right) \delta 5.76(1 \mathrm{H}, \mathrm{m}), 5.32(1 \mathrm{H}, \mathrm{d}, J=15.5 \mathrm{~Hz}), 5.24$ $(1 \mathrm{H}, \mathrm{d}, J=10.5 \mathrm{~Hz}), 3.48(1 \mathrm{H}, \mathrm{dt}, J=8.0,6.0 \mathrm{~Hz}), 1.65-1.62(2 \mathrm{H}, \mathrm{m}), 0.94(3 \mathrm{H}, \mathrm{t}, J=7.5 \mathrm{~Hz}) ;{ }^{13} \mathrm{C} \mathrm{NMR}$ $\left(100 \mathrm{MHz}, \mathrm{CDCl}_{3}\right) \delta 132.5,116.6,57.1,17.6,8.40$.

\section{2-Methyl-allylamine hydrochloride (20i).}




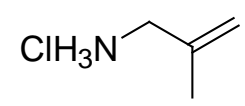

(Commercially available) Flash Chromatography (Gradient of 5-15\% $\mathrm{MeOH}$ in $\mathrm{CH}_{2} \mathrm{Cl}_{2}$ ) yielded a white solid (87\%). $\mathrm{R}_{f}=0.12\left(\mathrm{MeOH}: \mathrm{CH}_{2} \mathrm{Cl}_{2} ; 5: 95\right) ;{ }^{1} \mathrm{H} \mathrm{NMR}\left(300 \mathrm{MHz}, \mathrm{CD}_{3} \mathrm{OD}\right) \delta 5.17(1 \mathrm{H}, \mathrm{s}), 5.09(1 \mathrm{H}$, s), $3.57(2 \mathrm{H}, \mathrm{s}), 1.91(3 \mathrm{H}, \mathrm{s}) ;{ }^{13} \mathrm{C} \mathrm{NMR}\left(100 \mathrm{MHz}, \mathrm{CDCl}_{3}\right) \delta 137.9,113.5,44.1,19.2$.

(E)-But-2-enylamine hydrochloride (20j).

$$
\mathrm{ClH}_{3} \mathrm{~N}
$$

Flash Chromatography (Gradient of 5-15\% MeOH in $\mathrm{CH}_{2} \mathrm{Cl}_{2}$ ) yielded a white solid (85\%). $\mathrm{R}_{f}=0.12$ (MeOH: $\left.\mathrm{CH}_{2} \mathrm{Cl}_{2} ; 5: 95\right) ;{ }^{1} \mathrm{H}$ NMR (300 MHz, $\left.\mathrm{CD}_{3} \mathrm{OD}\right) \delta 5.95(1 \mathrm{H}, \mathrm{dqt}, J=15.0,6.5,1.5 \mathrm{~Hz}), 5.58(1 \mathrm{H}$, dtq, $J=15.5,7.0,1.5 \mathrm{~Hz}), 3.47(2 \mathrm{H}, \mathrm{dd}, J=7.0,1.5 \mathrm{~Hz}), 1.76(3 \mathrm{H}, \mathrm{dd}, J=6.5,1.5 \mathrm{~Hz}) ;{ }^{13} \mathrm{C}$ NMR $(100$ $\left.\mathrm{MHz}, \mathrm{CDCl}_{3}\right) \delta 135.1,123.7,42.5,18.1$.

(E)-1-Methyl-pent-2-enylamine hydrochloride (201).

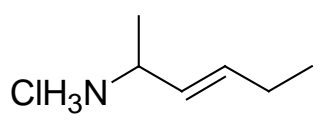

(Turk, S.D.; Louthan, R.P.; Cobb, R.L.; Bresson, C.R. J. Org. Chem. 1962, (15), 2846-2853). Flash Chromatography (Gradient of $5-15 \% \mathrm{MeOH}$ in $\mathrm{CH}_{2} \mathrm{Cl}_{2}$ ) yielded a white solid (79\%). $\mathrm{R}_{f}=0.10$ (MeOH: $\left.\mathrm{CH}_{2} \mathrm{Cl}_{2} ; 5: 95\right) ;{ }^{1} \mathrm{H}$ NMR (300 MHz, $\left.\mathrm{CD}_{3} \mathrm{OD}\right) \delta 5.98(1 \mathrm{H}, \mathrm{dt}, J=15.5,6.5 \mathrm{~Hz}), 5.56(1 \mathrm{H}, \mathrm{ddt}, J$ $=15.5,7.5,1.5 \mathrm{~Hz}), 3.88(1 \mathrm{H}, \mathrm{dq}, J=7.0,6.5 \mathrm{~Hz}), 2.17(2 \mathrm{H}, \mathrm{dq}, J=6.5,7.5 \mathrm{~Hz}), 1.42(3 \mathrm{H}, \mathrm{d}, J=6.5$ $\mathrm{Hz}), 1.09(3 \mathrm{H}, \mathrm{t}, J=7.5 \mathrm{~Hz}) ;{ }^{13} \mathrm{C} \mathrm{NMR}\left(100 \mathrm{MHz}, \mathrm{CDCl}_{3}\right) \delta 137.4,125.8,49.08,24.9,18.3,12.1$.

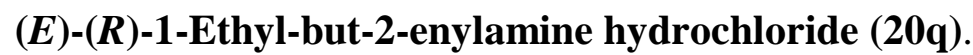

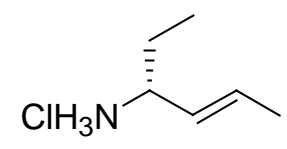

Flash Chromatography (Gradient of 5-15\% $\mathrm{MeOH}$ in $\mathrm{CH}_{2} \mathrm{Cl}_{2}$ ) yielded a white solid $(78 \%)$. $[\alpha]_{\mathrm{D}}=+6.5^{\circ}$ $\left(\mathrm{H}_{2} \mathrm{O}, \mathrm{c}=1.0\right) ; \mathrm{R}_{f}=0.10\left(\mathrm{MeOH}: \mathrm{CH}_{2} \mathrm{Cl}_{2} ; 5: 95\right) ;{ }^{1} \mathrm{H} \mathrm{NMR}\left(300 \mathrm{MHz}, \mathrm{CD}_{3} \mathrm{OD}\right) \delta 5.95(1 \mathrm{H}, \mathrm{dqd}, J=15.5$, 6.5, $1.0 \mathrm{~Hz}), 5.44(1 \mathrm{H}, \mathrm{ddq}, J=15.5,6.5,1.5 \mathrm{~Hz}), 3.58(1 \mathrm{H}, \mathrm{dt}, J=8.5,6.5 \mathrm{~Hz}), 1.81(3 \mathrm{H}, \mathrm{dd}, J=6.5$, $1.5 \mathrm{~Hz}), 1.80-1.60(2 \mathrm{H}, \mathrm{m}), 0.99(3 \mathrm{H}, \mathrm{t}, J=7.5 \mathrm{~Hz}) ;{ }^{13} \mathrm{C} \mathrm{NMR}\left(100 \mathrm{MHz}, \mathrm{CDCl}_{3}\right) \delta 132.8,126.4,55.1$, $26.2,16.6,8.8$.

Preparation of chiral tosyl iminodiazaphospholidine. In a flame dried round bottom flask under $\mathrm{N}_{2}$, allylic alcohol $(0.6 \mathrm{mmol})$ was added to a stirring solution of chiral phospholidine $\mathbf{2 3}(0.6 \mathrm{mmol})$ in 
benzene $(6 \mathrm{~mL})$. The reaction was stirred for 6 hours under a dynamic atmosphere of $\mathrm{N}_{2}$ then checked

for completion by ${ }^{31} \mathrm{P}$ NMR. Tosyl azide $(0.6 \mathrm{mmol})$ was then slowly added to the stirring solution resulting in rapid effervescence. After azide addition, the reaction was stirred for $45 \mathrm{~min}$ and concentrated in vacuo to yield a crude syrup. The crude syrup was then dissolved in a minimum amount of $\mathrm{CH}_{2} \mathrm{Cl}_{2}$ and purified by column chromatography.

$(R, R)-(E)-N$-(1,3-Dimethyl-2-pent-2-enyloxy-octahydro-2 $\lambda^{5}$-benzo[1,3,2]diazaphosphol-2-ylidene)4-methyl-benzenesulfonamide (24a).

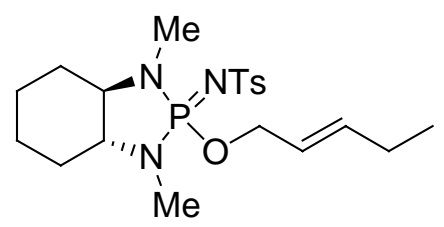

Flash chromatography (EtOAc:hexanes;1:4) yielded a clear oil $(85 \%) .(R, R)$ isomer: $[\alpha]_{\mathrm{D}}=-98.8^{\circ}$ $\left(\mathrm{CHCl}_{3}, \mathrm{c}=0.9\right) ; \mathrm{R}_{f}=0.32$ (EtOAc:hexanes; 1:4); IR (Thin Film) v 2936, 1445, 1276, 1171, 1143, 1017 $\mathrm{cm}^{-1} ;{ }^{1} \mathrm{H}$ NMR $\left(300 \mathrm{MHz}, \mathrm{CDCl}_{3}\right) \delta 7.83(2 \mathrm{H}, \mathrm{d}, J=8.5 \mathrm{~Hz}), 7.19(2 \mathrm{H}, \mathrm{d}, J=8.0 \mathrm{~Hz}), 5.80(1 \mathrm{H}, \mathrm{dt}, J=$ 15.0, $6.5 \mathrm{~Hz}), 5.52(1 \mathrm{H}, \mathrm{dtt}, J=15.5,6.5,1.5 \mathrm{~Hz}), 4.46(2 \mathrm{H}, \mathrm{dd}, J=11.0,7.5 \mathrm{~Hz}), 3.02-2.92(1 \mathrm{H}, \mathrm{m})$, 2.68-2.58 (1H, m), $2.62(3 \mathrm{H}, \mathrm{d}, J=11.5 \mathrm{~Hz}), 2.37(3 \mathrm{H}, \mathrm{s}), 2.15(3 \mathrm{H}, \mathrm{d}, J=11.5 \mathrm{~Hz}), 2.10-1.98(2 \mathrm{H}, \mathrm{m})$, 1.94-1.78 (2H, m), 1.44-1.10 (6H, m), $0.98(3 \mathrm{H}, \mathrm{t}, J=7.5 \mathrm{~Hz}) ;{ }^{13} \mathrm{C} \mathrm{NMR}\left(100 \mathrm{MHz}, \mathrm{CDCl}_{3}\right) \delta 143.4$, 140.9, 138.5, 128.7, 126.4, 123.6 (d, $J=5.5 \mathrm{~Hz}), 69.2$ (d, J=7.5 Hz), 63.7 (d, $J=10.0 \mathrm{~Hz}), 29.1(\mathrm{~d}, J=$ $10.0 \mathrm{~Hz}), 28.2(\mathrm{~d}, J=10.0 \mathrm{~Hz}), 27.6(\mathrm{~d}, J=2.0 \mathrm{~Hz}), 27.5,25.3,24.2,24.1,21.5,13.1 ;{ }^{31} \mathrm{P}$ NMR $(121$ $\mathrm{MHz}, \mathrm{CDCl}_{3}$ ) $\delta$ 26.98; MS (ESI) m/e (rel intensity) 448 (5), 358 (100), 205 (30), 187 (20); HRMS (ESI) $m / e$ calcd $\left(\mathrm{MNa}^{+}\right) 448.1794$, found 448.1806 .

$(R, R)-(Z)-N$-(1,3-Dimethyl-2-pent-2-enyloxy-octahydro-2 $\lambda^{5}$-benzo[1,3,2]diazaphosphol-2-ylidene)4-methyl-benzenesulfonamide (24c).

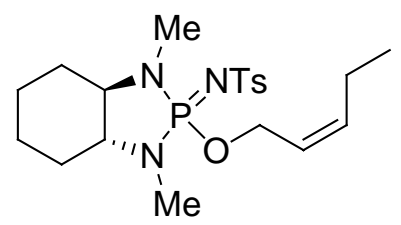

Flash chromatography (EtOAc:hexanes;1:4) yielded a clear oil $(75 \%)(R, R)$ isomer: $[\alpha]_{\mathrm{D}}=-89.6^{\circ}$ $\left(\mathrm{CHCl}_{3}, \mathrm{c}=1.0\right) ; \mathrm{R}_{f}=0.33$ (EtOAc:hexanes; 1:4); IR (Thin Film) v 2934, 1448, 1273, 1142, $992 \mathrm{~cm}^{-1}$; ${ }^{1} \mathrm{H}$ NMR $\left(300 \mathrm{MHz}, \mathrm{CDCl}_{3}\right) \delta 7.82(2 \mathrm{H}, \mathrm{d}, J=8.0 \mathrm{~Hz}), 7.19(2 \mathrm{H}, \mathrm{d}, J=8.0 \mathrm{~Hz}), 5.58(1 \mathrm{H}, \mathrm{dt}, J=11.0$, $7.0 \mathrm{~Hz}), 5.46(1 \mathrm{H}, \mathrm{dt}, J=11.0,7.0 \mathrm{~Hz}), 4.55(2 \mathrm{H}, \mathrm{m}), 2.93(1 \mathrm{H}, \mathrm{dt}, J=11.5,8.5 \mathrm{~Hz}), 2.68-2.62(1 \mathrm{H}, \mathrm{m})$, 
$2.62(3 \mathrm{H}, \mathrm{d}, J=11.5 \mathrm{~Hz}), 2.36(3 \mathrm{H}, \mathrm{s}), 2.12(3 \mathrm{H}, \mathrm{d}, J=11.5 \mathrm{~Hz}), 2.10-1.80(4 \mathrm{H}, \mathrm{m}), 1.40-1.08(6 \mathrm{H}, \mathrm{m})$, $0.96(3 \mathrm{H}, \mathrm{t}, J=7.5 \mathrm{~Hz}) ;{ }^{13} \mathrm{C} \mathrm{NMR}\left(100 \mathrm{MHz}, \mathrm{CDCl}_{3}\right) \delta 143.2(\mathrm{~d}, J=3.0 \mathrm{~Hz}), 140.9,137.2,128.7$, 126.3, 123.5 (d, $J=5.5 \mathrm{~Hz}), 64.0(\mathrm{~d}, J=7.5 \mathrm{~Hz}), 63.7$ (d, $J=10.0 \mathrm{~Hz}), 63.6$ (d, $J=11.0 \mathrm{~Hz}), 29.1$ (d, $J$ = 3.0 Hz), $28.1(\mathrm{~d}, J=9.0 \mathrm{~Hz}), 27.5(\mathrm{~d}, J=10.0 \mathrm{~Hz}), 27.4(\mathrm{~d}, J=2.0 \mathrm{~Hz}), 24.2,24.0,21.4,21.0,14.1$;

${ }^{31} \mathrm{P}$ NMR (121 MHz, $\mathrm{CDCl}_{3}$ ) $\delta$ 27.57; MS (ESI) m/e (rel intensity) 448 (18), 426 (3), 358 (100), 257 (10), 241 (10), 179 (17); HRMS (ESI) m/e calcd $\left(\mathrm{MNa}^{+}\right)$448.1794, found 448.1803.

\section{$(R, R)-(E)-\left(1,3-D i m e t h y l-2-p e n t-2-e n y l o x y-o c t a h y d r o-2 \lambda^{5}\right.$-benzo[1,3,2]diazaphosphol-2-ylidene)-} phosphoramidic acid diphenyl ester (24b).

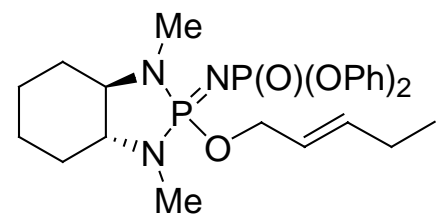

Flash chromatography (EtOAc:hexanes;1:3) yielded a clear oil $(73 \%) .(R, R)$ isomer: $[\alpha]_{\mathrm{D}}=-25.2^{\circ}$ $(\mathrm{CHCl3}, \mathrm{c}=1.3) ; \mathrm{R}_{f}=0.36$ (EtOAc:hexanes; 1:2); IR (Thin Film) v 2934, 1593, 1488, 1253, 1200, 997 $\mathrm{cm}^{-1} ;{ }^{1} \mathrm{H}$ NMR $\left(300 \mathrm{MHz}, \mathrm{CDCl}_{3}\right) \delta$ 7.32-7.20 (8H, m), 7.08-7.02 (2H, m), $5.69(1 \mathrm{H}, \mathrm{dt}, J=15.5,6.0$ Hz), $5.46(1 \mathrm{H}, \mathrm{dtt}, J=15.5,6.5,1.5 \mathrm{~Hz}), 4.40-4.28(2 \mathrm{H}, \mathrm{m}), 2.72-2.64(1 \mathrm{H}, \mathrm{m}), 2.62-2.54(1 \mathrm{H}, \mathrm{m}), 2.44$ $(3 \mathrm{H}, \mathrm{d}, J=12.5 \mathrm{~Hz}), 2.33(3 \mathrm{H}, \mathrm{d}, J=12.0 \mathrm{~Hz}), 2.01(2 \mathrm{H}, \mathrm{dt}, J=7.0,7.0 \mathrm{~Hz}), 1.94-1.86(2 \mathrm{H}, \mathrm{m}), 1.82-$ $1.74(2 \mathrm{H}, \mathrm{m}), 1.32-1.05(4 \mathrm{H}, \mathrm{m}), 0.96(3 \mathrm{H}, \mathrm{t}, J=7.5 \mathrm{~Hz}) ;{ }^{13} \mathrm{C} \mathrm{NMR}\left(100 \mathrm{MHz}, \mathrm{CDCl}_{3}\right) \delta 152.3(\mathrm{~d}, J=$ $7.0 \mathrm{~Hz}), 137.5,129.0,128.8,123.8(\mathrm{~d}, J=6.0 \mathrm{~Hz}), 123.4(\mathrm{~d}, J=8.0 \mathrm{~Hz}), 120.5$ (d, $J=12.0 \mathrm{~Hz}), 120.3$ $(\mathrm{d}, J=12.0 \mathrm{~Hz}), 68.3(\mathrm{~d}, J=8.5 \mathrm{~Hz}), 63.9(\mathrm{~d}, J=10.5 \mathrm{~Hz}), 63.2(\mathrm{~d}, J=10.0 \mathrm{~Hz}), 28.9(\mathrm{~d}, J=2.0 \mathrm{~Hz})$, $28.0(\mathrm{~d}, J=10.0 \mathrm{~Hz}), 27.6,27.5(\mathrm{~d}, J=10.0 \mathrm{~Hz}), 25.0,23.9(\mathrm{~d}, J=10.0 \mathrm{~Hz}), 13.0 ;{ }^{31} \mathrm{P} \mathrm{NMR}(121 \mathrm{MHz}$, $\left.\mathrm{CDCl}_{3}\right) \delta 27.80(\mathrm{~d}, J=55.5 \mathrm{~Hz}),-10.00(\mathrm{~d}, J=55.5 \mathrm{~Hz}) ;$ MS (ESI) $m / e$ (rel intensity) 526 (12), 504 (11), 436 (100); HRMS (ESI) m/e calcd $\left(\mathrm{MH}^{+}\right)$504.2175, found 504.2164.

\section{$(R, R)-(Z)-\left(1,3-D i m e t h y l-2-p e n t-2-e n y l o x y-o c t a h y d r o-2 \lambda^{5}\right.$-benzo[1,3,2]diazaphosphol-2-ylidene)-} phosphoramidic acid diphenyl ester(24d).

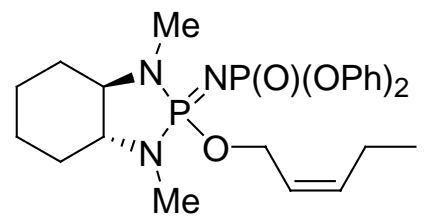

Flash chromatography (EtOAc:hexanes;1:2) yielded a clear oil $(81 \%) .(R, R)$ isomer: $[\alpha]_{\mathrm{D}}=-31.8^{\circ}$ $\left(\mathrm{CHCl}_{3}, \mathrm{c}=1.4\right) ; \mathrm{R}_{f}=0.35$ (EtOAc:hexanes; 1:2); IR (Thin Film) v 2935, 1594, 1490, 1253, 1202, 996, $913 \mathrm{~cm}^{-1} ;{ }^{1} \mathrm{H}$ NMR $\left(300 \mathrm{MHz}, \mathrm{CDCl}_{3}\right) \delta$ 7.32-7.23 (8H, m), 7.08-7.04 (2H, m), $5.56(1 \mathrm{H}, \mathrm{dt}, J=11.0$, 
$7.0 \mathrm{~Hz}), 5.43(1 \mathrm{H}, \mathrm{dt}, J=11.0,7.0 \mathrm{~Hz}), 4.46(2 \mathrm{H}, \mathrm{dd}, J=10.5,7.0 \mathrm{~Hz}), 2.74-2.67(1 \mathrm{H}, \mathrm{m}), 2.62-2.54$ $(1 \mathrm{H}, \mathrm{m}), 2.47(3 \mathrm{H}, \mathrm{d}, J=11.0 \mathrm{~Hz}), 2.35(3 \mathrm{H}, \mathrm{d}, J=11.0 \mathrm{~Hz}), 2.03(2 \mathrm{H}, \mathrm{dq}, J=7.0,7.0 \mathrm{~Hz}), 1.96-1.90$ $(2 \mathrm{H}, \mathrm{m}), 1.82-1.75(2 \mathrm{H}, \mathrm{m}), 1.32-1.08(4 \mathrm{H}, \mathrm{m}), 0.96(3 \mathrm{H}, \mathrm{t}, J=7.5 \mathrm{~Hz}) ;{ }^{13} \mathrm{C} \mathrm{NMR}\left(100 \mathrm{MHz}, \mathrm{CDCl}_{3}\right)$ $\delta 152.5(\mathrm{~d}, J=7.5 \mathrm{~Hz}), 136.5,129.2,124.1(\mathrm{~d}, J=6.0 \mathrm{~Hz}), 123.7(\mathrm{~d}, J=8.5 \mathrm{~Hz}), 120.6(2 \mathrm{~d}, J=12.0$ Hz), 64.1 (d, $J=10.5 \mathrm{~Hz}), 63.5(2 \mathrm{~d}, J=10.0 \mathrm{~Hz}), 29.2$ (d, $J=3.0 \mathrm{~Hz}), 28.3(\mathrm{~d}, J=10.0 \mathrm{~Hz}), 27.9(2 \mathrm{~d}, J$ $=5.0 \mathrm{~Hz}), 24.1(\mathrm{~d}, J=13.0 \mathrm{~Hz}), 21.0,14.1 ;{ }^{31} \mathrm{P} \mathrm{NMR}\left(121 \mathrm{MHz}, \mathrm{CDCl}_{3}\right) \delta 28.00(\mathrm{~d}, J=55.5 \mathrm{~Hz})$, 10.00 (d, $J=56.0 \mathrm{~Hz}$ ); MS (ESI) m/e (rel intensity) 504 (6), 436 (100), 487 (19), 526 (3); HRMS (ESI) $m / e$ calcd $\left(\mathrm{MH}^{+}\right)$504.2175, found 504.2148.

\section{$(R, R)-(E)-N$-(1,3-Diisopropyl-2-pent-2-enyloxy-octahydro-2 $\lambda^{5}$-benzo[1,3,2]diazaphosphol-2-}

ylidene)-4-methyl-benzenesulfonamide (24e).

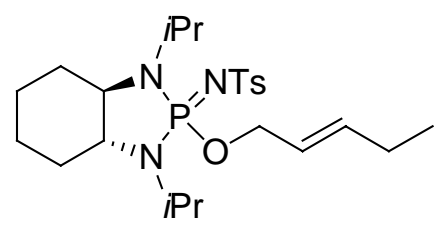

Flash chromatography (EtOAc:hexanes;1:4) yielded a clear oil $(80 \%)$. $(R, R)$ isomer: $[\alpha]_{\mathrm{D}}=-51.2^{\circ}$ $\left(\mathrm{CHCl}_{3}, \mathrm{c}=1.0\right) ; \mathrm{R}_{f}=0.35$ (EtOAc:hexanes; 1:4); IR (Thin Film) v 2936, 2870, 1268, 1203, 1175, 1020 $\mathrm{cm}^{-1} ;{ }^{1} \mathrm{H}$ NMR $\left(300 \mathrm{MHz}, \mathrm{CDCl}_{3}\right) \delta 7.86(2 \mathrm{H}, \mathrm{d}, J=8.0 \mathrm{~Hz}), 7.21(2 \mathrm{H}, \mathrm{d}, J=8.5 \mathrm{~Hz}), 5.83(1 \mathrm{H}, \mathrm{dt}, J=$ 15.5, 7.0 Hz), $5.58(1 \mathrm{H}, \mathrm{dt}, J=15.5,6.5 \mathrm{~Hz}), 4.53(2 \mathrm{H}, \mathrm{dd}, J=8.0,8.0 \mathrm{~Hz}), 3.63-3.36(2 \mathrm{H}, \mathrm{m}), 3.28-$ $3.19(1 \mathrm{H}, \mathrm{m}), 2.99-2.91(1 \mathrm{H}, \mathrm{m}), 2.39$ (3H, s), 2.13-2.06 (4H, m), 1.86-1.76 (2H, m), 1.54-1.18 (4H, m), $1.36(3 \mathrm{H}, \mathrm{d}, J=7.0 \mathrm{~Hz}), 1.22(3 \mathrm{H}, \mathrm{d}, J=7.0 \mathrm{~Hz}), 1.17(3 \mathrm{H}, \mathrm{d}, J=7.0 \mathrm{~Hz}), 1.07(3 \mathrm{H}, \mathrm{d}, J=7.0 \mathrm{~Hz})$, $1.01(3 \mathrm{H}, \mathrm{t}, J=7.0 \mathrm{~Hz}) ;{ }^{13} \mathrm{C} \mathrm{NMR}\left(100 \mathrm{MHz}, \mathrm{CDCl}_{3}\right) \delta 143.8(\mathrm{~d}, J=6.0 \mathrm{~Hz}), 140.6,137.9,128.8$, 125.9, $123.7(\mathrm{~d}, J=7.0 \mathrm{~Hz}), 69.2(\mathrm{~d}, J=9.0 \mathrm{~Hz}), 60.5(\mathrm{~d}, J=10.5 \mathrm{~Hz}), 59.7(\mathrm{~d}, J=11.5 \mathrm{~Hz}), 44.8(\mathrm{~d}, J$ $=4.5 \mathrm{~Hz}), 44.6(\mathrm{~d}, J=3.0 \mathrm{~Hz}), 30.6(\mathrm{~d}, J=12.0 \mathrm{~Hz}), 30.4(\mathrm{~d}, J=10.0 \mathrm{~Hz}), 25.3,24.4,24.1(\mathrm{~d}, J=2.0$ $\mathrm{Hz}), 22.9(\mathrm{~d}, J=7.0 \mathrm{~Hz}), 22.7(\mathrm{~d}, J=4.0 \mathrm{~Hz}), 21.4,19.7,19.6(\mathrm{~d}, J=2.0 \mathrm{~Hz}), 13.1$; ${ }^{31} \mathrm{P}$ NMR $(121$ $\left.\mathrm{MHz}, \mathrm{CDCl}_{3}\right) \delta 1$ 19.31; MS (EI) m/e (rel intensity) 481 (1), 440 (6), 412 (100), 398 (10), 370 (43), 328 (28), 243 (28), 217 (47), 203 (11), 175 (38), 138 (25); HRMS (EI) m/e calcd $\left(\mathrm{M}^{+}\right.$) 481.2528, found 481.2500 .

$(R, R)-(E)-\left(1,3-D i i s o p r o p y l-2-p e n t-2\right.$-enyloxy-octahydro-2 $\lambda^{5}$-benzo[1,3,2] diazaphosphol-2-ylidene)phosphoramidic acid diphenyl ester (24f). 


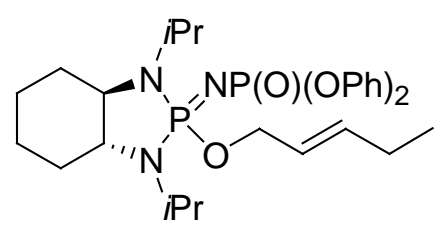

Flash chromatography (EtOAc:hexanes;1:2) yielded a clear oil $(78 \%) .(R, R)$ isomer: $[\alpha]_{\mathrm{D}}=-29.7^{\circ}$ $(\mathrm{CHCl} 3, \mathrm{c}=0.7) ; \mathrm{R}_{f}=0.42$ (EtOAc:hexanes; 2:1); IR (Thin Film) v 2933, 1594, 1489, 1299, 1202, 1003, $910 \mathrm{~cm}^{-1} ;{ }^{1} \mathrm{H}$ NMR $\left(300 \mathrm{MHz}, \mathrm{CDCl}_{3}\right) \delta$ 7.32-7.22 (8H, m), 7.09-7.02 (2H, m), $5.68(1 \mathrm{H}, \mathrm{dt}, J=15.5$, $6.5 \mathrm{~Hz}), 5.46(1 \mathrm{H}, \mathrm{dtt}, J=15.5,6.5,1.0 \mathrm{~Hz}), 4.34(2 \mathrm{H}, \mathrm{dd}, J=9.5,7.0 \mathrm{~Hz}), 3.54-3.30(2 \mathrm{H}, \mathrm{m}), 3.08-$ $2.96(1 \mathrm{H}, \mathrm{m}), 2.94-2.83(1 \mathrm{H}, \mathrm{m}), 2.10-1.98(4 \mathrm{H}, \mathrm{m}), 1.80-1.72(2 \mathrm{H}, \mathrm{m}), 1.44-1.12(16 \mathrm{H}, \mathrm{m}), 0.97(3 \mathrm{H}, \mathrm{t}$, $J=7.5 \mathrm{~Hz}) ;{ }^{13} \mathrm{C}$ NMR $\left(100 \mathrm{MHz}, \mathrm{CDCl}_{3}\right) \delta 152.7(\mathrm{~d}, J=8.0 \mathrm{~Hz}), 137.4,129.2,124.2(\mathrm{~d}, J=6.5 \mathrm{~Hz})$, 123.6, $120.7(2 \mathrm{~d}, J=5.0 \mathrm{~Hz}), 68.8(\mathrm{~d}, J=8.5 \mathrm{~Hz}), 59.9(2 \mathrm{~d}, J=11.0 \mathrm{~Hz}), 44.7(\mathrm{~d}, J=3.0 \mathrm{~Hz}), 44.4(\mathrm{~d}$, $J=3.0 \mathrm{~Hz}), 30.9(\mathrm{~d}, J=11.5 \mathrm{~Hz}), 30.2(\mathrm{~d}, J=10.5 \mathrm{~Hz}), 25.3,24.3(\mathrm{~d}, J=14.0 \mathrm{~Hz}), 23.4(\mathrm{~d}, J=7.0 \mathrm{~Hz})$, $22.0(\mathrm{~d}, J=4.0 \mathrm{~Hz}), 19.6,13.2 ;{ }^{31} \mathrm{P}$ NMR $\left(121 \mathrm{MHz}, \mathrm{CDCl}_{3}\right) \delta 19.70(\mathrm{~d}, J=57.0 \mathrm{~Hz}),-12.30(\mathrm{~d}, J=$ $56.5 \mathrm{~Hz}$ ); MS (ESI) m/e (rel intensity) 582 (7), 560 (9), 492 (100), 450 (8); HRMS (ESI) m/e calcd $\left(\mathrm{MH}^{+}\right)$560.2801, found 560.2824.

\section{$(R, R)$-(E)- $N$-(1,3-Dibenzyl-2-pent-2-enyloxy-octahydro-2 $\lambda^{5}$-benzo[1,3,2] diazaphosphol-2-ylidene)-}

\section{4-methyl-benzenesulfonamide (24g).}

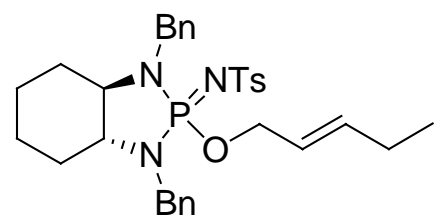

Flash chromatography (EtOAc:hexanes;1:4) yielded a clear oil $(85 \%) .(R, R)$ isomer: $[\alpha]_{\mathrm{D}}=-43.2^{\circ}$ $\left(\mathrm{CHCl}_{3}, \mathrm{c}=1.0\right) ; \mathrm{R}_{f}=0.40$ (EtOAc:hexanes; 1:4); IR (Thin Film) v 2937, 2869, 1496, 1454, 1273, 1092, $964 \mathrm{~cm}^{-1} ;{ }^{1} \mathrm{H}$ NMR $\left(300 \mathrm{MHz}, \mathrm{CDCl}_{3}\right) \delta 7.91(2 \mathrm{H}, \mathrm{d}, J=7.5 \mathrm{~Hz}), 7.38-7.20(12 \mathrm{H}, \mathrm{m}), 5.63(1 \mathrm{H}, \mathrm{dt}, J=$ 15.5, 6.0 Hz), $5.32(1 \mathrm{H}, \mathrm{dtt}, J=15.0,7.0,1.5 \mathrm{~Hz}), 4.55(1 \mathrm{H}, \mathrm{dd}, J=16.0,12.0 \mathrm{~Hz}), 4.36-4.29(1 \mathrm{H}, \mathrm{m})$, 4.17-4.09 (1H, m), $3.98(1 \mathrm{H}, \mathrm{dd}, J=16.0,13.0 \mathrm{~Hz}), 3.94-.89(2 \mathrm{H}, \mathrm{m}), 3.32-3.25(1 \mathrm{H}, \mathrm{m}), 2.98-2.90$ $(1 \mathrm{H}, \mathrm{m}), 2.39(3 \mathrm{H}, \mathrm{s}), 1.88(2 \mathrm{H}, \mathrm{dq}, J=6.5,6.5 \mathrm{~Hz}), 1.77-1.58(4 \mathrm{H}, \mathrm{m}), 1.28-0.98(4 \mathrm{H}, \mathrm{m}), 0.84(3 \mathrm{H}, \mathrm{t}$, $J=7.0 \mathrm{~Hz}) ;{ }^{13} \mathrm{C} \mathrm{NMR}\left(100 \mathrm{MHz}, \mathrm{CDCl}_{3}\right) \delta 143.3(\mathrm{~d}, J=4.5 \mathrm{~Hz}), 141.0,193.1(\mathrm{~d}, J=5.5 \mathrm{~Hz}), 138.6$, $137.3(\mathrm{~d}, J=3.0 \mathrm{~Hz}), 128.8,128.3(\mathrm{~d}, J=12.0 \mathrm{~Hz}), 127.8(\mathrm{~d}, J=10.0 \mathrm{~Hz}), 127.2,127.1,126.1,123.3$ $(\mathrm{d}, J=7.0 \mathrm{~Hz}), 69.8(\mathrm{~d}, J=9.5 \mathrm{~Hz}), 64.7(\mathrm{~d}, J=9.5 \mathrm{~Hz}), 61.8(\mathrm{~d}, J=11.0 \mathrm{~Hz}), 47.5(\mathrm{~d}, J=6.0 \mathrm{~Hz})$, $45.5(\mathrm{~d}, J=3.0 \mathrm{~Hz}), 29.5(\mathrm{~d}, J=10.0 \mathrm{~Hz}), 28.2(\mathrm{~d}, J=9.0 \mathrm{~Hz}), 25.0,24.1(\mathrm{~d}, J=8.0 \mathrm{~Hz}), 21.4,21.8 ;{ }^{31} \mathrm{P}$ NMR (121 MHz, CDCl ${ }_{3}$ ) $\delta$ 25.77; MS (EI) m/e (rel intensity) 577 (2), 508 (16), 418 (11), 355 (12), 265 (16), 91 (100); HRMS (EI) m/e calcd $\left(\mathrm{M}^{+}\right)$577.2528, found 577.2544. 
$(R, R)-(E)-\left(1,3-D i b e n z y l-2-p e n t-2-e n y l o x y-o c t a h y d r o-2 \lambda^{5}\right.$-benzo[1,3,2] diazaphosphol-2-ylidene)phosphoramidic acid diphenyl ester (24h).

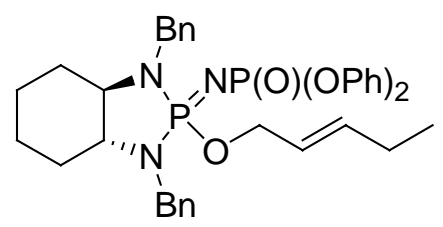

Flash chromatography (EtOAc:hexanes;1:4) yielded a clear oil $(82 \%) .(R, R)$ isomer: $[\alpha]_{\mathrm{D}}=-30.6^{\circ}$ $\left(\mathrm{CHCl}_{3}, \mathrm{c}=0.7\right) ; \mathrm{R}_{f}=0.35$ (EtOAc:hexanes; 1:2); IR (Thin Film) v 2933, 1588, 1487, 1198, 1004,911 $\mathrm{cm}^{-1} ;{ }^{1} \mathrm{H}$ NMR $\left(300 \mathrm{MHz}, \mathrm{CDCl}_{3}\right) \delta$ 7.38-7.36 (2H, m), 7.33-7.18 (16H, m), 7.08-7.04 (2H, m), 5.54 $(1 \mathrm{H}, \mathrm{dtt}, J=15.5,6.0,1.0 \mathrm{~Hz}), 5.25(1 \mathrm{H}, \mathrm{dtt}, J=15.5,6.5,1.5 \mathrm{~Hz}), 4.40(1 \mathrm{H}, \mathrm{dd}, J=15.0,12.0 \mathrm{~Hz})$, 4.21-4.13 (2H, m), 4.02-3.93 (2H, m), 3.85 (1H, dd, $J=16.0,13.0 \mathrm{~Hz}), 3.10-3.03$ (1H, m), 2.96-2.88 $(1 \mathrm{H}, \mathrm{m}), 1.87(2 \mathrm{H}, \mathrm{dt}, J=6.5,6.5 \mathrm{~Hz}), 1.74-1.56(4 \mathrm{H}, \mathrm{m}), 1.20-1.00(4 \mathrm{H}, \mathrm{m}), 0.85(3 \mathrm{H}, \mathrm{t}, J=7.0 \mathrm{~Hz})$; ${ }^{13} \mathrm{C} \mathrm{NMR}\left(100 \mathrm{MHz}, \mathrm{CDCl}_{3}\right) \delta 152.6(\mathrm{~d}, J=17.0 \mathrm{~Hz}), 139.4(\mathrm{~d}, J=5.0 \mathrm{~Hz}), 138.5(\mathrm{~d}, J=5.5 \mathrm{~Hz})$, 138.0, 129.4, $128.5(\mathrm{~d}, J=17.0 \mathrm{~Hz}), 128.0(\mathrm{~d}, J=12.5 \mathrm{~Hz}), 127.1(\mathrm{~d}, J=17.0 \mathrm{~Hz}), 123.8(\mathrm{~d}, J=11.0$ $\mathrm{Hz}), 120.8(\mathrm{~d}, J=5.0 \mathrm{~Hz}), 120.6(\mathrm{~d}, J=5.0 \mathrm{~Hz}), 69.2(\mathrm{~d}, J=9.0 \mathrm{~Hz}), 64.8(\mathrm{~d}, J=11.0 \mathrm{~Hz}), 62.7(\mathrm{~d}, J=$ $11.0 \mathrm{~Hz}), 47.5(\mathrm{~d}, J=4.0 \mathrm{~Hz}), 46.3$ (d, $J=4.0 \mathrm{~Hz}), 29.8$ (d, $J=10.0 \mathrm{~Hz}), 28.9$ (d, $J=10.0 \mathrm{~Hz}), 25.2$, 24.3, 24.2, 13.1; ${ }^{31} \mathrm{P}$ NMR (121 MHz, $\left.\mathrm{CDCl}_{3}\right) \delta 26.40(\mathrm{~d}, J=54.5 \mathrm{~Hz}),-10.20$ (d, $\left.J=55.0 \mathrm{~Hz}\right)$; MS (ESI) m/e (rel intensity) 678 (14), 656 (14), 588 (55), 457 (36), 363 (57), 188 (100); HRMS (ESI) m/e calcd $\left(\mathrm{MH}^{+}\right)$656.2801, found 656.2797.

Diastereoselective rearrangement of tosyl iminodiazaphospholidines. In a flame dried round bottom flask under $\mathrm{N}_{2}$, a solution of iminodiazaphospholidine $(0.6 \mathrm{mmol})$ in $\mathrm{CH}_{2} \mathrm{Cl}_{2}(5 \mathrm{~mL})$ was added to $8 \mathrm{mg}$ of bis(acetonitrile)dichloropalladium(II) $(5 \mathrm{~mol} \%)$. The reaction was stirred for 24 hours under an atmosphere of $\mathrm{N}_{2}$ then checked for completion by ${ }^{31} \mathrm{P}$ NMR. The reaction was then concentrated in vacuo, dissolved in a minimum amount of $\mathrm{CH}_{2} \mathrm{Cl}_{2}$, and purified by column chromatography.

$(4 R, 5 R)-N$-(1,3-Dimethyl-2-oxo-octahydro-2 $\lambda^{5}$-benzo[1,3,2]diazaphosphol-2-yl)- $N$-(1-ethyl-allyl)-4methyl-benzenesulfonamide (25a).

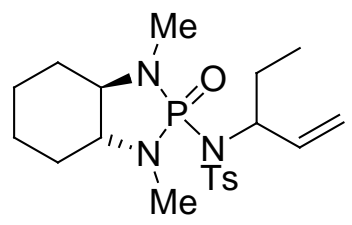


Flash chromatography (EtOAc:hexanes;1:4) yielded a clear oil $\left(83 \%, 3.5: 1 \mathrm{dr}\right.$ and 81\%, 3.5:1 dr). $\mathrm{R}_{f}=$ 0.40 (EtOAc:hexanes; 1:5); IR (Thin Film) v 2936, 2867, 1448, 1344, 1264, 1219, 1167, 1091, 1007 , $926 \mathrm{~cm}^{-1} ;{ }^{1} \mathrm{H}$ NMR $\left(300 \mathrm{MHz}, \mathrm{CDCl}_{3}\right) \delta 7.86-7.78(4.5 \mathrm{H}, \mathrm{m}), 7.30(1 \mathrm{H}, \mathrm{d}, J=7.0 \mathrm{~Hz}), 7.29(3.5 \mathrm{H}, \mathrm{d}, J$ $=7.5 \mathrm{~Hz}), 6.40-6.18(4.5 \mathrm{H}, \mathrm{m}), 5.34(1 \mathrm{H}, \mathrm{d}, J=17.0 \mathrm{~Hz}), 5.23(1 \mathrm{H}, \mathrm{dd}, J=10.0,1.5 \mathrm{~Hz}), 4.95-4.78$ $(3.5 \mathrm{H}, \mathrm{m}), 4.60-4.42$ (3.5H, m), 4.30-4.02 (4.5H, m), 3.30-3.18 (4.5H, m), 2.74-2.26 (4.5H, m), 2.62 $(10.5 \mathrm{H}, \mathrm{d}, J=12.0 \mathrm{~Hz}), 2.56(3 \mathrm{H}, \mathrm{d}, J=12.0 \mathrm{~Hz}), 2.44(3 \mathrm{H}, \mathrm{s}), 2.42(10.5 \mathrm{H}, \mathrm{s}), 2.23(3 \mathrm{H}, \mathrm{d}, J=12.0$ Hz), $2.16(10.5 \mathrm{H}, \mathrm{d}, J=12.0 \mathrm{~Hz}), 2.04-1.80(18 \mathrm{H}, \mathrm{m}), 1.50-1.14(27 \mathrm{H}, \mathrm{m}), 0.90(10.5 \mathrm{H}, \mathrm{t}, J=6.5 \mathrm{~Hz})$, $0.87(3 \mathrm{H}, \mathrm{m}) ;{ }^{13} \mathrm{C}$ NMR $\left(100 \mathrm{MHz}, \mathrm{CDCl}_{3}\right) \delta 143.6,138.8,138.5,137.7,129.3,129.1,128.6,128.2$, 127.8, 126.3, 119.1, 117.9, $68.4(\mathrm{~d}, J=8.0 \mathrm{~Hz}), 66.5,66.0,64.7(\mathrm{~d}, J=10.0 \mathrm{~Hz}), 64.5(\mathrm{~d}, J=10.0 \mathrm{~Hz})$, 62.0, 29.2, 28.9, $28.6(\mathrm{~d}, J=10.0 \mathrm{~Hz}), 28.5(\mathrm{~d}, J=10.0 \mathrm{~Hz}), 28.0,27.8(\mathrm{~d}, J=5.2 \mathrm{~Hz}), 27.7(\mathrm{~d}, J=5.2$ $\mathrm{Hz}), 24.5,24.3,22.3,21.7,21.5,12.0,11.8 ;{ }^{31} \mathrm{P} \mathrm{NMR}\left(121 \mathrm{MHz}, \mathrm{CDCl}_{3}\right) \delta 24.31,23.96$; MS (ESI) $m / e$ (rel intensity) 448 (5), 426 (7), 380 (3), 358 (100), 205 (20), 187 (46); HRMS (ESI) m/e calcd (MNa ) 426.1974, found 426.1969.

$(4 R, 5 R)-\left(1,3-D i m e t h y l-2-o x o-o c t a h y d r o-2 \lambda^{5}\right.$-benzo[1,3,2] diazaphosphol-2-yl)-pent-2-enylphosphoramidic acid diphenyl ester (25b).

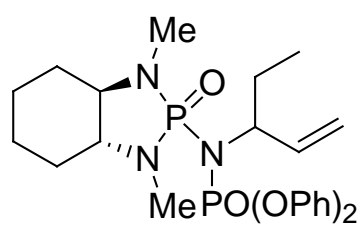

Flash chromatography (EtOAc:hexanes;1:4) yielded a clear oil (27\%, 1.7:1 dr and 35\%, 3.5:1 dr). $\mathrm{R}_{f}=$ 0.32 (EtOAc:hexanes; 1:5); IR (Thin Film) v 2936, 1570, 1477, 1274, 1220, 1195, $935 \mathrm{~cm}^{-1}$; ${ }^{1} \mathrm{H}$ NMR (300 MHz, $\left.\mathrm{CDCl}_{3}\right) \delta$ 7.38-7.25 (8H, m), 7.22-7.13 (2H, m), 6.50-6.34 (1H, m), 5.30-5.20 (2H, m), 4.13$4.00(1 \mathrm{H}, \mathrm{m}), 3.35-3.10(1 \mathrm{H}, \mathrm{m}), 2.75-2.68(1 \mathrm{H}, \mathrm{m}), 2.55(3 \mathrm{H}, \mathrm{d}, J=11.5 \mathrm{~Hz}), 2.35(3 \mathrm{H}, \mathrm{d}, J=11.5$ $\mathrm{Hz}), 2.20-1.90(2 \mathrm{H}, \mathrm{m}), 1.85-1.72(4 \mathrm{H}, \mathrm{m}), 1.40-1.18(4 \mathrm{H}, \mathrm{m}), 0.95(3 \mathrm{H}, \mathrm{t}, J=7.5 \mathrm{~Hz}) ;{ }^{13} \mathrm{C}$ NMR $(100$ $\left.\mathrm{MHz}, \mathrm{CDCl}_{3}\right) \delta 150.9(\mathrm{~d}, J=7.0 \mathrm{~Hz}), 150.7(\mathrm{~d}, J=7.0 \mathrm{~Hz}), 139.0,129.7,129.5,129.4,125.2,124.8$, 120.5, 118.5, 138.9, 129.7, 129.5, 129.4, 65.2, 64.2 (d, $J=9.0 \mathrm{~Hz}), 62.9(\mathrm{~d}, J=9.0 \mathrm{~Hz}), 28.6(\mathrm{~d}, J=$ $10.0 \mathrm{~Hz}), 28.1,27.8(\mathrm{~d}, J=10.0 \mathrm{~Hz}), 24.4,24.1,13.3,12.0 ;{ }^{31} \mathrm{P} \mathrm{NMR}\left(121 \mathrm{MHz}, \mathrm{CDCl}_{3}\right) \delta 25.65(\mathrm{~d}, J=$ $20.0 \mathrm{~Hz}),-4.43(\mathrm{~d}, J=20.5 \mathrm{~Hz})$; HRMS (EI) $m / e$ calcd $\left(\mathrm{M}^{+}\right)$504.2175, found 504.2177.

(4R,5R)-(1,3-Dimethyl-2-oxo-octahydro-2 $\lambda^{5}$-benzo[1,3,2] diazaphosphol-2-yl)-(1-ethyl-allyl)phosphoramidic acid diphenyl ester (25b - side product). 


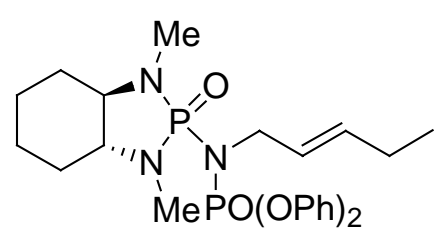

Flash chromatography (EtOAc:hexanes;1:4) yielded a clear oil (40-50\%). $\mathrm{R}_{f}=0.36$ (EtOAc:hexanes; 1:5); IR (Thin Film) v 2935, 1590, 1485, 1275, 1220, 1180, 103, $940 \mathrm{~cm}^{-1} ;{ }^{1} \mathrm{H}$ NMR (300 MHz, $\mathrm{CDCl}_{3}$ ) $\delta$ 7.38-7.28 (10H, m), 7.22-7.15 (2H, m), $5.84(1 \mathrm{H}, \mathrm{dt}, J=16.0,6.5 \mathrm{~Hz}), 5.68(1 \mathrm{H}, \mathrm{dtt}, J=16.0,7.0,1.5$ $\mathrm{Hz}), 4.24-4.02(2 \mathrm{H}, \mathrm{m}), 3.22-3.14(2 \mathrm{H}, \mathrm{m}), 2.74-2.68(1 \mathrm{H}, \mathrm{m}), 2.47(3 \mathrm{H}, \mathrm{d}, J=11.5 \mathrm{~Hz}), 2.30(3 \mathrm{H}, \mathrm{d}, J$ $=11.5 \mathrm{~Hz}), 2.06(2 \mathrm{H}, \mathrm{dq}, J=7.5 \mathrm{~Hz}), 1.98-1.93(2 \mathrm{H}, \mathrm{m}), 1.85-1.79(4 \mathrm{H}, \mathrm{m}), 1.40-1.18(4 \mathrm{H}, \mathrm{m}), 0.98$ $(3 \mathrm{H}, \mathrm{t}, J=7.5 \mathrm{~Hz}) ;{ }^{13} \mathrm{C} \mathrm{NMR}\left(100 \mathrm{MHz}, \mathrm{CDCl}_{3}\right) \delta 150.8(\mathrm{~d}, J=5.5 \mathrm{~Hz}), 13638.0,129.7,129.5,129.2$, 125.9, 125.2, 124.9, 120.6 (d, $J=3.5 \mathrm{~Hz}), 120.5(\mathrm{~d}, J=3.5 \mathrm{~Hz}), 64.2(\mathrm{~d}, J=10.0 \mathrm{~Hz}), 63.0(\mathrm{~d}, J=10.0$ $\mathrm{Hz}), 50.1,28.6,28.5,28.4,27.9,27.8,27.8(\mathrm{~d}, J=10.4 \mathrm{~Hz}), 25.3,24.4,24.2,13.3 ;{ }^{31} \mathrm{P} \mathrm{NMR}(121 \mathrm{MHz}$, $\left.\mathrm{CDCl}_{3}\right) \delta 25.40(\mathrm{~d}, J=20.5 \mathrm{~Hz}),-4.32(\mathrm{~d}, J=20.5 \mathrm{~Hz})$; HRMS $(\mathrm{EI}) \mathrm{m} / e$ calcd $\left(\mathrm{M}^{+}\right) 504.2175$, found 504.2168 .

\section{(4R,5R)- $N$-(1,3-Diisopropyl-2-oxo-octahydro-2 $\lambda^{5}$-benzo[1,3,2] diazaphosphol-2-yl)- $N$-(1-ethyl-} allyl)-4-methyl-benzenesulfonamide (25e).

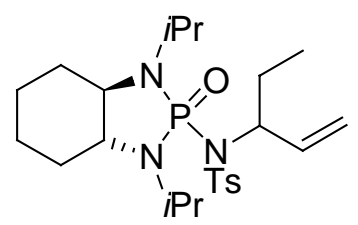

Flash chromatography (EtOAc:hexanes;1:4) yielded a clear oil $(80 \%, 2.5: 1 \mathrm{dr}) . \quad \mathrm{R}_{f}=0.36$ (EtOAc:hexanes; 1:5); IR (Thin Film) v 2935, 1599, 1455, 1302, 1251, 1162, 1008, $928 \mathrm{~cm}^{-1}$; ${ }^{1} \mathrm{H}$ NMR $\left(300 \mathrm{MHz}, \mathrm{CDCl}_{3}\right) \delta 7.87(4 \mathrm{H}, \mathrm{d}, J=8.0 \mathrm{~Hz}), 7.29-7.25(4 \mathrm{H}, \mathrm{m}), 6.41-6.21(2 \mathrm{H}, \mathrm{m}), 5.16(1 \mathrm{H}, \mathrm{d}, J=$ $10.0 \mathrm{~Hz}), 5.10(1 \mathrm{H}, \mathrm{d}, J=17.0 \mathrm{~Hz}), 4.96-4.86(1 \mathrm{H}, \mathrm{m}), 4.60-4.34(1 \mathrm{H}, \mathrm{m}), 4.04-3.30(8 \mathrm{H}, \mathrm{m}), 3.10-3.28$ $(2 \mathrm{H}, \mathrm{m}), 2.42(6 \mathrm{H}, \mathrm{s}), 2.22-2.02(6 \mathrm{H}, \mathrm{m}), 1.82-1.74(4 \mathrm{H}, \mathrm{m}), 1.48-1.06(34 \mathrm{H}, \mathrm{m}), 0.79(3 \mathrm{H}, \mathrm{t}, J=6.5$ $\mathrm{Hz}), 0.70-0.60(3 \mathrm{H}, \mathrm{m}) ;{ }^{13} \mathrm{C} \mathrm{NMR}\left(100 \mathrm{MHz}, \mathrm{CDCl}_{3}\right) \delta 143.4,138.2,129.0,128.6,128.5,128.3,125.8$, 118.6, 118.1, $66.4(\mathrm{~d}, J=3.0 \mathrm{~Hz}), 66.3,60.9(\mathrm{~d}, J=4.0 \mathrm{~Hz}), 60.8(\mathrm{~d}, J=4.0 \mathrm{~Hz}), 58.0,44.8(\mathrm{~d}, J=7.0$ Hz), 43.7 (d, $J=7.0 \mathrm{~Hz}), 43.6$ (d, $J=5.0 \mathrm{~Hz}), 32.1$ (d, $J=10.0 \mathrm{~Hz}), 30.8$ (d, $J=10.0 \mathrm{~Hz}), 30.6,28.0$, 27.4, 25.3, 25.1, 24.5 (d, $J=12.0 \mathrm{~Hz}), 23.2,21.5,19.6,19.4,12.0,11.9 ;{ }^{31} \mathrm{P}$ NMR (121 MHz, $\left.\mathrm{CDCl}_{3}\right)$ $\delta$ 18.96; MS (EI) m/e (rel intensity) 482 (29), 438 (47), 414 (30), 398 (36), 370 (63), 326 (49), 243 (100), 217 (42), 175 (27), 138 (30), 91 (42); HRMS (EI) m/e calcd $\left(\mathrm{M}^{+}\right)$481.2528, found 481.2529. 
(4R,5R)-(1,3-Diisopropyl-2-oxo-octahydro-2 $\lambda^{5}$-benzo[1,3,2]diazaphosphol-2-yl)-(1-ethyl-allyl)phosphoramidic acid diphenyl ester (25f).

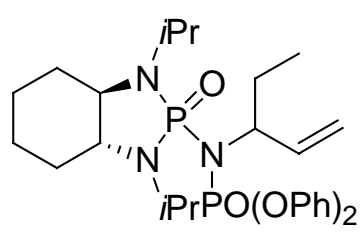

Flash chromatography (EtOAc:hexanes;1:4) yielded a clear oil $(77 \%, \quad 1.5: 1 \mathrm{dr}) . \quad \mathrm{R}_{f}=0.40$ (EtOAc:hexanes; 1:5); IR (Thin Film) v 2935, 1592, 1491, 1288, 1219, 1174, 1023, $931 \mathrm{~cm}^{-1}$; ${ }^{1} \mathrm{H}$ NMR (300 MHz, $\left.\mathrm{CDCl}_{3}\right) \delta$ 7.33-7.20 (12H, m), 7.16-7.08 (8H, m), 6.62-6.38 (2H, m), 5.36-5.18 (4H, m), 4.18-4.00 (2H, m), 3.64-3.32 (4H, m), 3.60-2.95 (2H, m), 2.50-2.36 (2H, m), 2.12-1.60 (12H, m), 1.48$1.30(8 \mathrm{H}, \mathrm{m}), 1.24-1.02(24 \mathrm{H}, \mathrm{m}), 0.92(6 \mathrm{H}, \mathrm{t}, J=6.5 \mathrm{~Hz}) ;{ }^{13} \mathrm{C} \mathrm{NMR}\left(100 \mathrm{MHz}, \mathrm{CDCl}_{3}\right) \delta 151.1,138.9$, 129.6, 129.6, $125.0(\mathrm{~d}, J=7.5 \mathrm{~Hz}), 121.0(\mathrm{~d}, J=3.0 \mathrm{~Hz}), 121.0(\mathrm{~d}, J=3.0 \mathrm{~Hz}), 120.9,118.5,117.9$, 65.8, 65.4, $60.5(\mathrm{~d}, J=11.0 \mathrm{~Hz}), 60.4,59.3,44.4,43.7(\mathrm{~d}, J=6.0 \mathrm{~Hz}), 32.3(\mathrm{~d}, J=9.5 \mathrm{~Hz}), 32.2(\mathrm{~d}, J=$ $10.0 \mathrm{~Hz}), 31.4(\mathrm{~d}, J=11.5 \mathrm{~Hz}), 31.2(\mathrm{~d}, J=11.5 \mathrm{~Hz}), 29.9,28.5,28.3,25.4(\mathrm{~d}, J=3.0 \mathrm{~Hz}), 25.3(\mathrm{~d}, J=$ $3.0 \mathrm{~Hz}), 24.7,24.4,23.8(\mathrm{~d}, J=9.5 \mathrm{~Hz}), 23.4(\mathrm{~d}, J=9.5 \mathrm{~Hz}), 19.5$ (d, $J=7.0 \mathrm{~Hz}), 12.4,12.3 ;{ }^{31} \mathrm{P}$ NMR $\left(121 \mathrm{MHz}, \mathrm{CDCl}_{3}\right) \delta 17.00(\mathrm{~d}, J=20.0 \mathrm{~Hz}), 16.80(\mathrm{~d}, J=20.0 \mathrm{~Hz}),-8.20(\mathrm{~d}, J=20.0 \mathrm{~Hz}),-8.50(\mathrm{~d}, J=$ $20.0 \mathrm{~Hz}$ ); MS (EI) m/e (rel intensity) 559 (14), 516 (75), 490 (36), 448 (100), 353 (62), 294 (24), 157 (32), 138 (91); HRMS (ESI) m/e calcd $\left(\mathrm{MH}^{+}\right)$559.2729, found 559.2715.

$(4 R, 5 R)-N$-(1,3-Dibenzyl-2-oxo-octahydro-2 $\lambda^{5}$-benzo[1,3,2] diazaphosphol-2-yl)- $N$-(1-ethyl-allyl)-4methyl-benzenesulfonamide (25g).

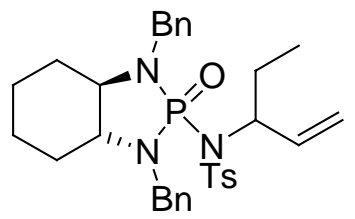

Flash chromatography (EtOAc:hexanes;1:4) yielded a clear oil $(73 \%, 1: 1 \mathrm{dr}) . \quad \mathrm{R}_{f}=0.35$ (EtOAc:hexanes; 1:5); IR (Thin Film) v 2933, 1453, 1341, 1221, 1165, 1007, $923 \mathrm{~cm}^{-1}$; ${ }^{1} \mathrm{H}$ NMR (300 $\left.\mathrm{MHz}, \mathrm{CDCl}_{3}\right) \delta$ 7.90-7.84 (4H, m), 7.60-7.45 (8H, m), 7.36-7.18 (16H, m), 6.48-6.25 (2H, m), 5.14 (1H, $\mathrm{d}, J=17.0 \mathrm{~Hz}), 5.14-5.08(1 \mathrm{H}, \mathrm{m}), 4.93(1 \mathrm{H}, \mathrm{d}, J=10.0 \mathrm{~Hz}), 4.72-4.62(1 \mathrm{H}, \mathrm{m}), 4.61(1 \mathrm{H}, \mathrm{d}, J=16.0$ Hz), $4.51(1 \mathrm{H}, \mathrm{d}, J=16.0 \mathrm{~Hz}), 4.30-4.12(2 \mathrm{H}, \mathrm{m}), 4.08-3.95(2 \mathrm{H}, \mathrm{m}), 3.92-3.71(3 \mathrm{H}, \mathrm{m}), 3.61-3.44(3 \mathrm{H}$, m), 2.84-2.70 (2H, m), 2.45 (3H, s), 2.42-2.24 (2H, m), 1.99-1.88 (2H, m), 1.88-1.78 (2H, m), 1.62-1.18 $(12 \mathrm{H}, \mathrm{m}), 1.08-0.88(2 \mathrm{H}, \mathrm{m}), 0.82(3 \mathrm{H}, \mathrm{t}, J=7.5 \mathrm{~Hz}), 0.69(3 \mathrm{H}, \mathrm{t}, J=7.0 \mathrm{~Hz}) ;{ }^{13} \mathrm{C} \mathrm{NMR}(100 \mathrm{MHz}$, $\left.\mathrm{CDCl}_{3}\right) \delta 144.1,140.8(\mathrm{~d}, J=6.0 \mathrm{~Hz}), 140.7(\mathrm{~d}, J=6.0 \mathrm{~Hz}), 138.9,138.6,136.6(\mathrm{~d}, J=1.0 \mathrm{~Hz}), 136.3$, 129.6, $129.5(\mathrm{~d}, J=6.0 \mathrm{~Hz}), 129.3,128.5(\mathrm{~d}, J=3.0 \mathrm{~Hz}), 128.4(\mathrm{~d}, J=6.0 \mathrm{~Hz}), 128.3(\mathrm{~d}, J=6.0 \mathrm{~Hz})$, 
128.1, 127.8, 127.7, 127.5, 127.0, 126.9, 119.2, 118.8, 67.0 (d, $J=1.5 \mathrm{~Hz}), 66.5$ (d, J=1.5 Hz), 63.5, $61.5(\mathrm{~d}, J=11.0 \mathrm{~Hz}), 60.6(\mathrm{~d}, J=11.0 \mathrm{~Hz}), 47.0(\mathrm{~d}, J=5.5 \mathrm{~Hz}), 46.8(\mathrm{~d}, J=5.5 \mathrm{~Hz}), 46.6(\mathrm{~d}, J=3.0$ Hz), $46.1(\mathrm{~d}, J=3.0 \mathrm{~Hz}), 30.5(\mathrm{~d}, J=10.0 \mathrm{~Hz}), 30.4(\mathrm{~d}, J=10.0 \mathrm{~Hz}), 29.8,28.6,28.3(\mathrm{~d}, J=10.0 \mathrm{~Hz})$, $28.0(\mathrm{~d}, J=10.0 \mathrm{~Hz}), 27.9,24.5,24.2(\mathrm{~d}, J=4.0 \mathrm{~Hz}), 21.7,12.1,11.8 ;{ }^{31} \mathrm{P} \mathrm{NMR}\left(121 \mathrm{MHz}, \mathrm{CDCl}_{3}\right)$ $\delta$ 20.82, 20.44; MS (ESI) m/e (rel intensity) 600 (35), 578 (30), 532 (20), 510 (100), 339 (15), 295 (12), 195 (91); HRMS (ESI) m/e calcd $\left(\mathrm{MH}^{+}\right)$578.2600, found 578.2616.

\section{$(4 R, 5 R)-\left(1,3-D i b e n z y l-2-o x o-o c t a h y d r o-2 \lambda^{5}\right.$-benzo[1,3,2]diazaphosphol-2-yl)-(1-ethyl-allyl)-} phosphoramidic acid diphenyl ester (25h).

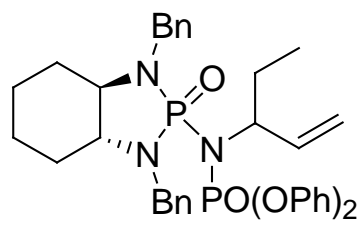

Flash chromatography (EtOAc:hexanes;1:4) yielded a clear oil $(85 \%, 1: 1 \mathrm{dr}) \cdot \mathrm{R}_{f}=0.40$ (EtOAc:hexanes; 1:5); IR (Thin Film) v 2937, 2868, 1591, 1489, 1455, 1289, 1219, 1191, 1007, $941 \mathrm{~cm}^{-1} ;{ }^{1} \mathrm{H}$ NMR (300 $\left.\mathrm{MHz}, \mathrm{CDCl}_{3}\right) \delta$ 7.60-7.52 (4H, m), 7.44-7.08 (32H, m), 6.60-6.42 (2H, m), 5.30-5.16 (4H, m), $4.36(1 \mathrm{H}$, $\mathrm{d}, J=15.0 \mathrm{~Hz}), 4.25(1 \mathrm{H}, \mathrm{d}, J=15.0 \mathrm{~Hz}), 4.20-4.01(3 \mathrm{H}, \mathrm{m}), 3.91(1 \mathrm{H}, \mathrm{d}, J=16.0 \mathrm{~Hz}), 3.80(1 \mathrm{H}, \mathrm{d}, J=$ $13.0 \mathrm{~Hz}), 3.60(1 \mathrm{H}, \mathrm{dd}, J=16.0,8.0 \mathrm{~Hz}), 3.52-3.34(2 \mathrm{H}, \mathrm{m}), 2.88-2.76(2 \mathrm{H}, \mathrm{m}), 2.50-2.32(2 \mathrm{H}, \mathrm{m})$, 1.88-1.42 (12H, m), 1.30-0.82 (8H, m), $0.89(6 \mathrm{H}, \mathrm{t}, J=6.5 \mathrm{~Hz}) ;{ }^{13} \mathrm{C} \mathrm{NMR}\left(100 \mathrm{MHz}, \mathrm{CDCl}_{3}\right) \delta 151.1$ $(\mathrm{d}, J=6.5 \mathrm{~Hz}), 151.0(\mathrm{~d}, J=7.0 \mathrm{~Hz}), 140.9(\mathrm{~d}, J=5.0 \mathrm{~Hz}), 140.7(\mathrm{~d}, J=5.0 \mathrm{~Hz}), 138.2,137.8,129.8$, $129.7(\mathrm{~d}, J=5.5 \mathrm{~Hz}), 128.8,128.4(\mathrm{~d}, J=2.0 \mathrm{~Hz}), 128.3(\mathrm{~d}, J=6.0 \mathrm{~Hz}), 127.5$ (d, $J=1.5 \mathrm{~Hz}), 127.2$, $126.9(\mathrm{~d}, J=6.0 \mathrm{~Hz}), 125.3(\mathrm{~d}, J=9.0 \mathrm{~Hz}), 125.2(\mathrm{~d}, J=10.0 \mathrm{~Hz}), 121.1(\mathrm{~d}, J=5.0 \mathrm{~Hz}), 120.8(\mathrm{~d}, J=$ $5.0 \mathrm{~Hz}), 118.9,118.3,65.9,65.6,64.5,62.9,61.8,47.0(\mathrm{~d}, J=3.0 \mathrm{~Hz}), 46.9(\mathrm{~d}, J=4.5 \mathrm{~Hz}), 46.7(\mathrm{~d}, J=$ $4.5 \mathrm{~Hz}), 46.5(\mathrm{~d}, J=4.0 \mathrm{~Hz}), 30.6$ (d, $J=9.5 \mathrm{~Hz}), 30.4$ (d, $J=9.5 \mathrm{~Hz}), 29.0$ (d, $J=10.5 \mathrm{~Hz}), 28.4$ (d, $J$ $=10.5 \mathrm{~Hz}), 24.3,12.3,12.1 ;{ }^{31} \mathrm{P} \mathrm{NMR}\left(121 \mathrm{MHz}, \mathrm{CDCl}_{3}\right) \delta 24.31,23.96 ; \mathrm{MS}$ (EI) $m / e$ (rel intensity) 656 (15), 586 (12), 564 (26), 496 (27), 394 (13), 339 (12), 247 (13), 186 (23), 91 (100); HRMS (EI) m/e calcd $\left(\mathrm{M}^{+}\right)$655.2729, found 655.2731. 

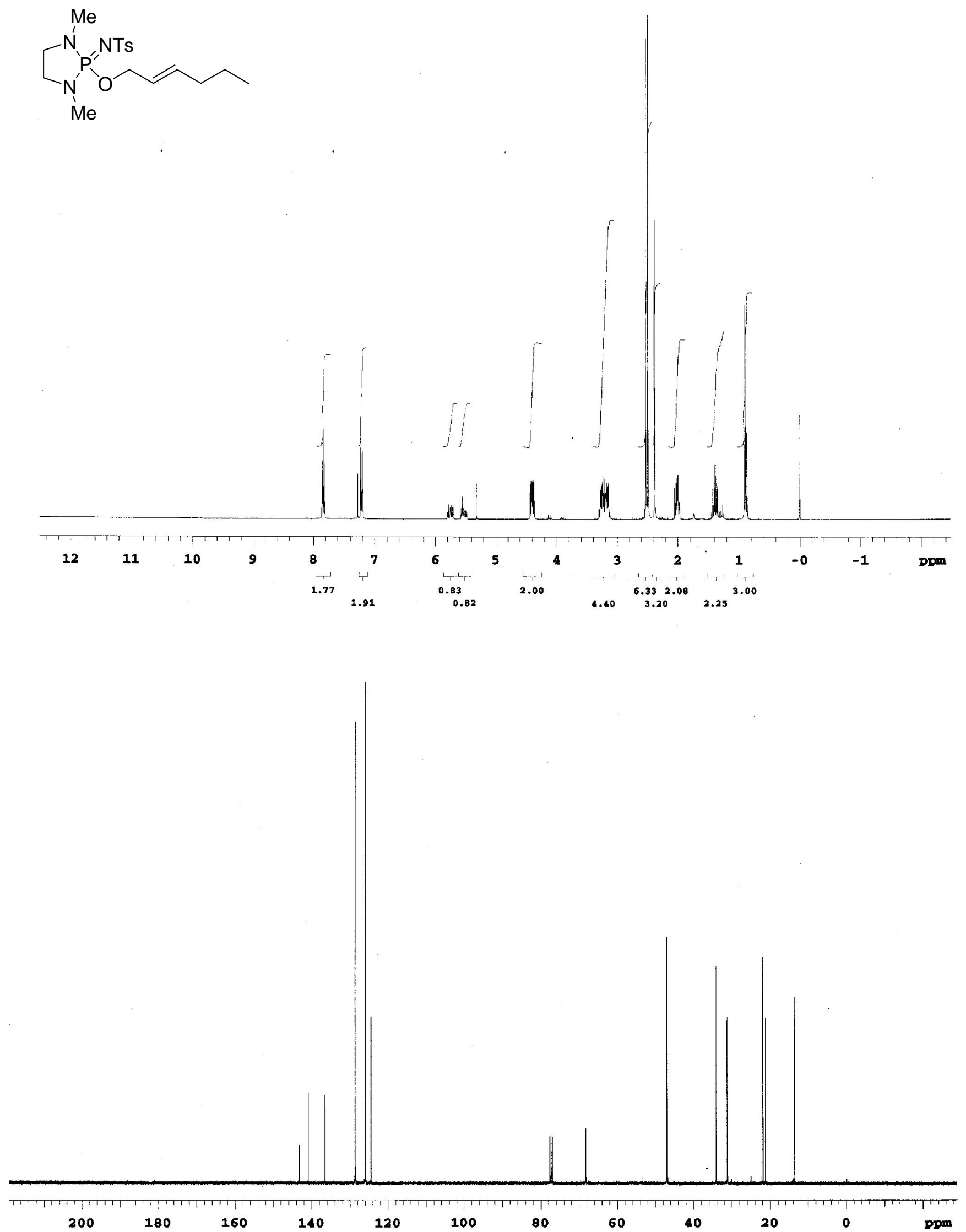

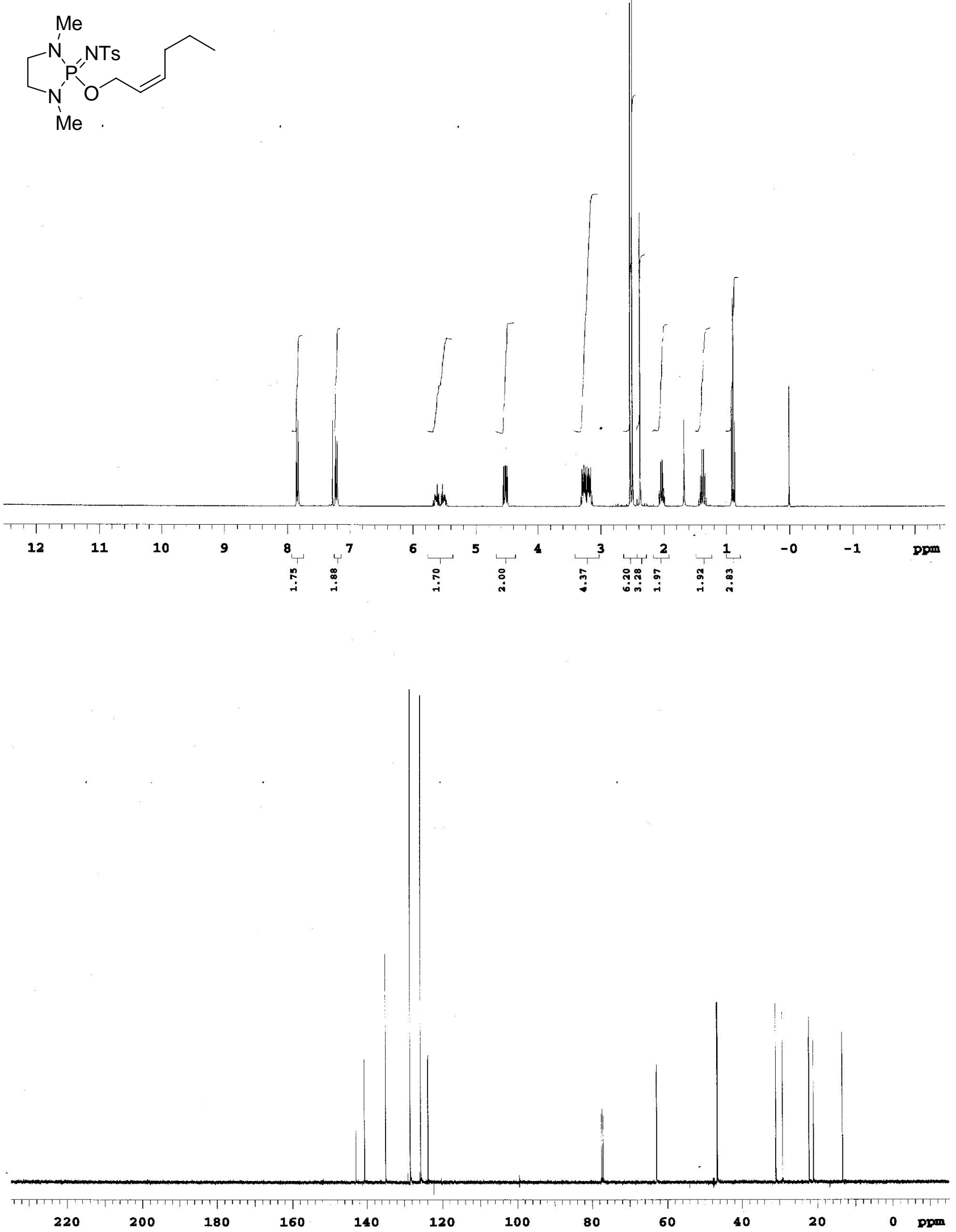

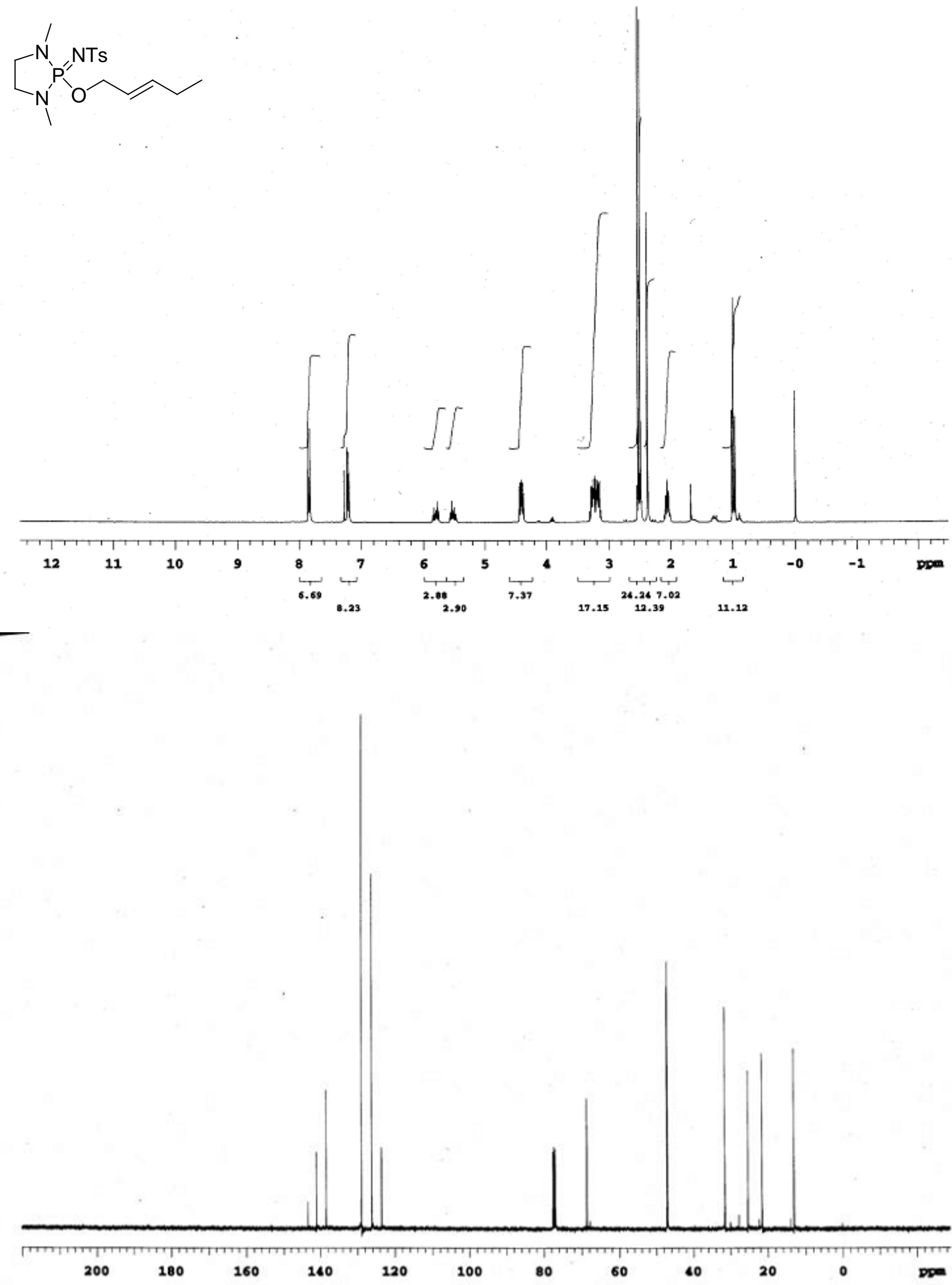
L
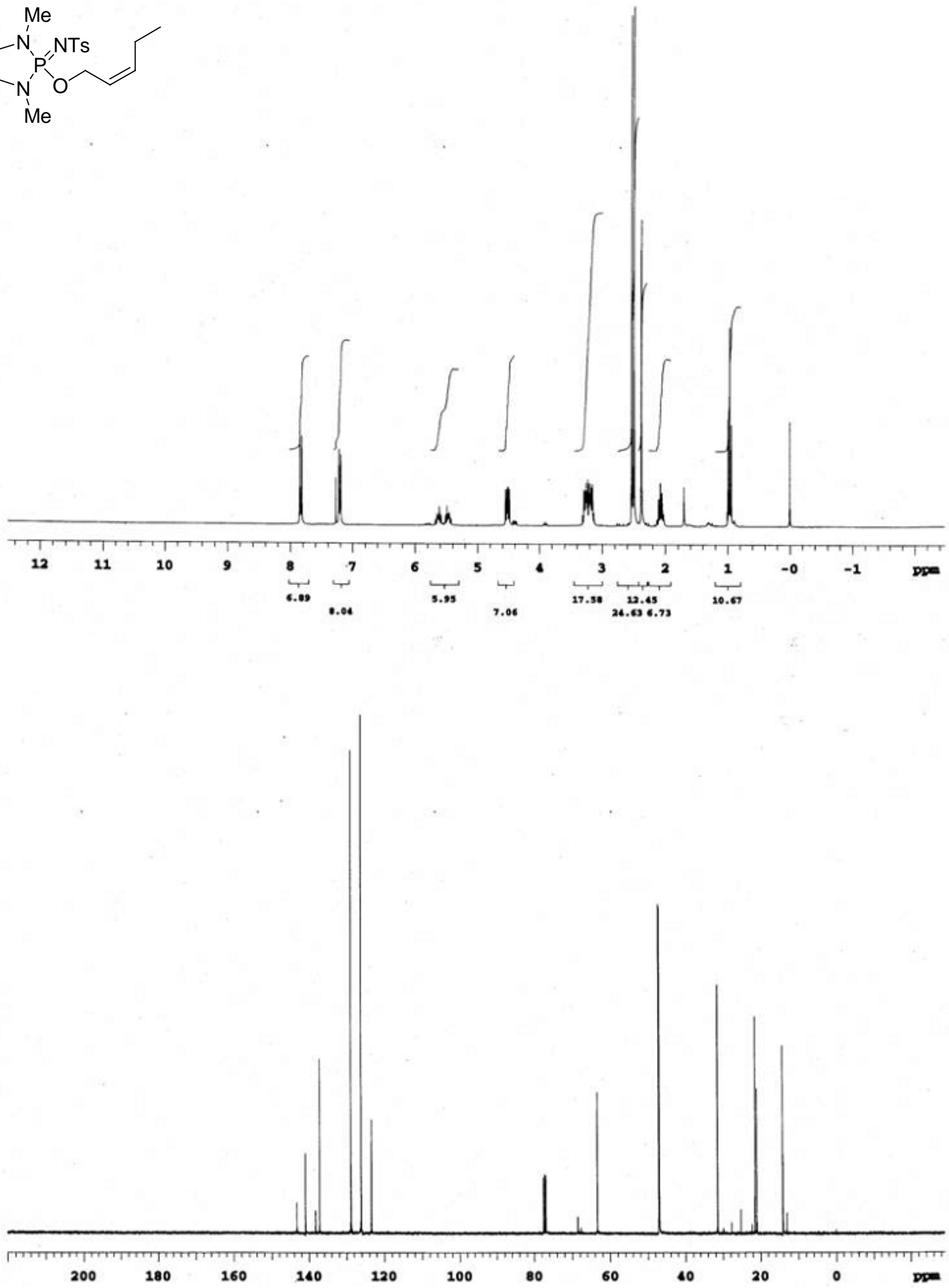

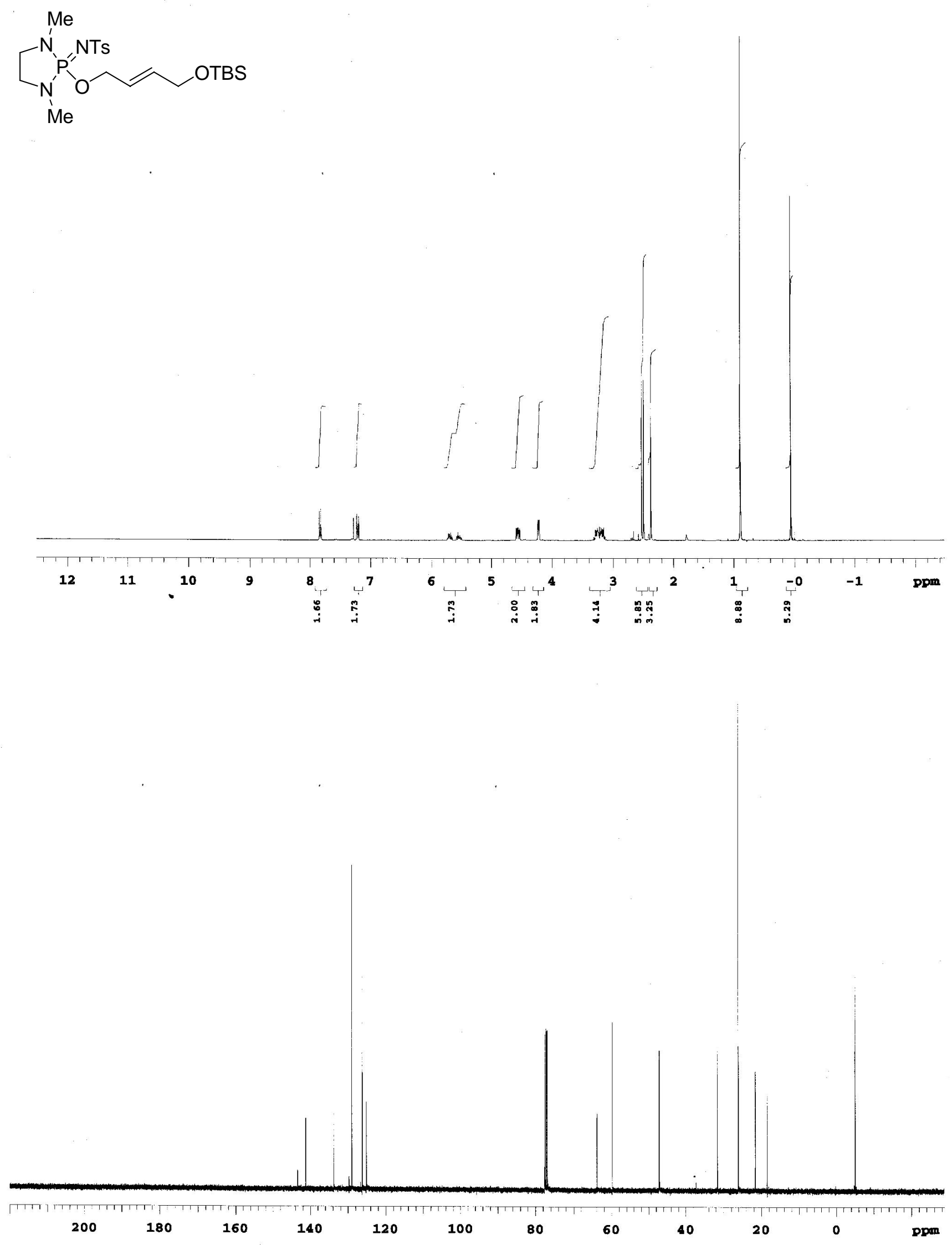

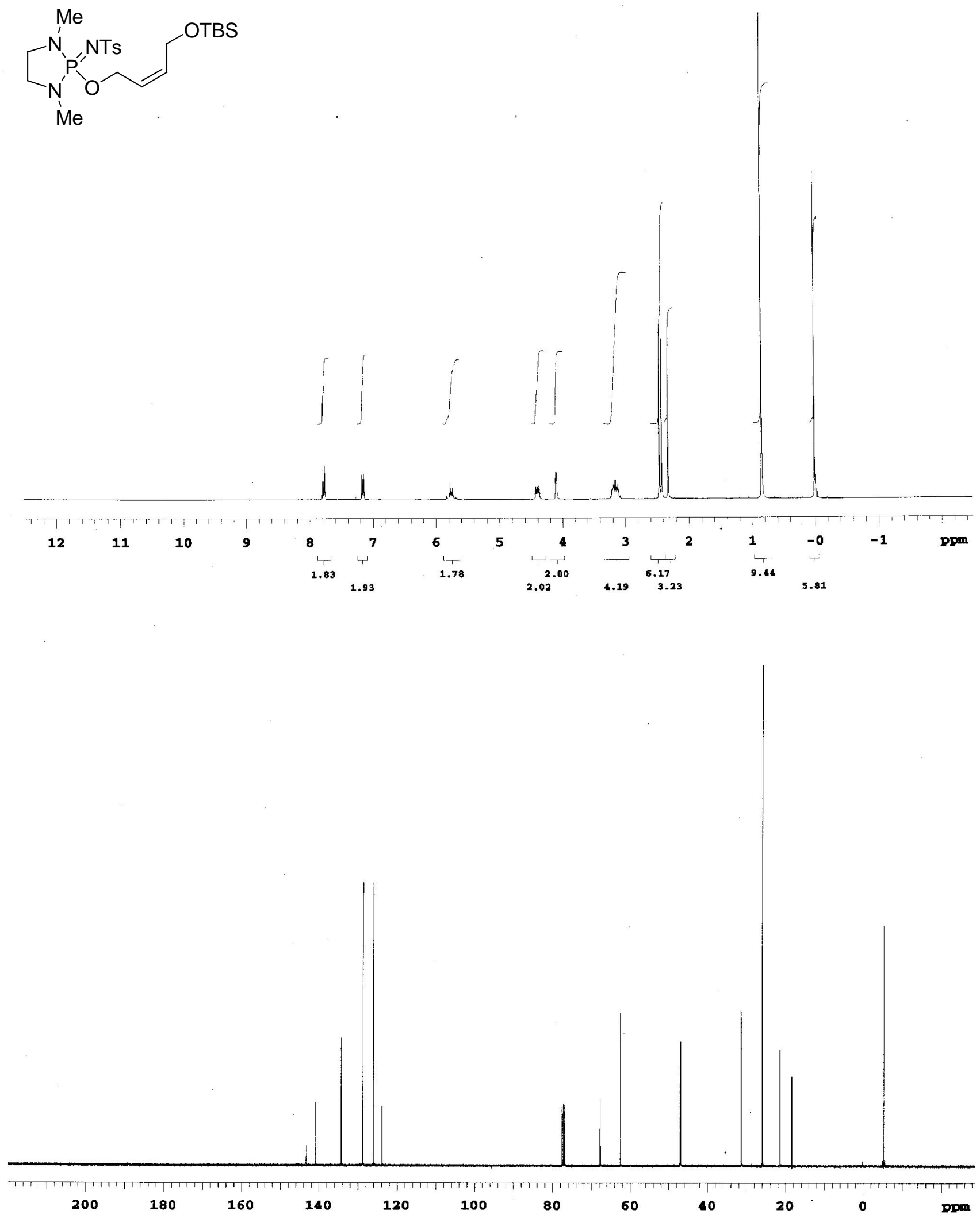

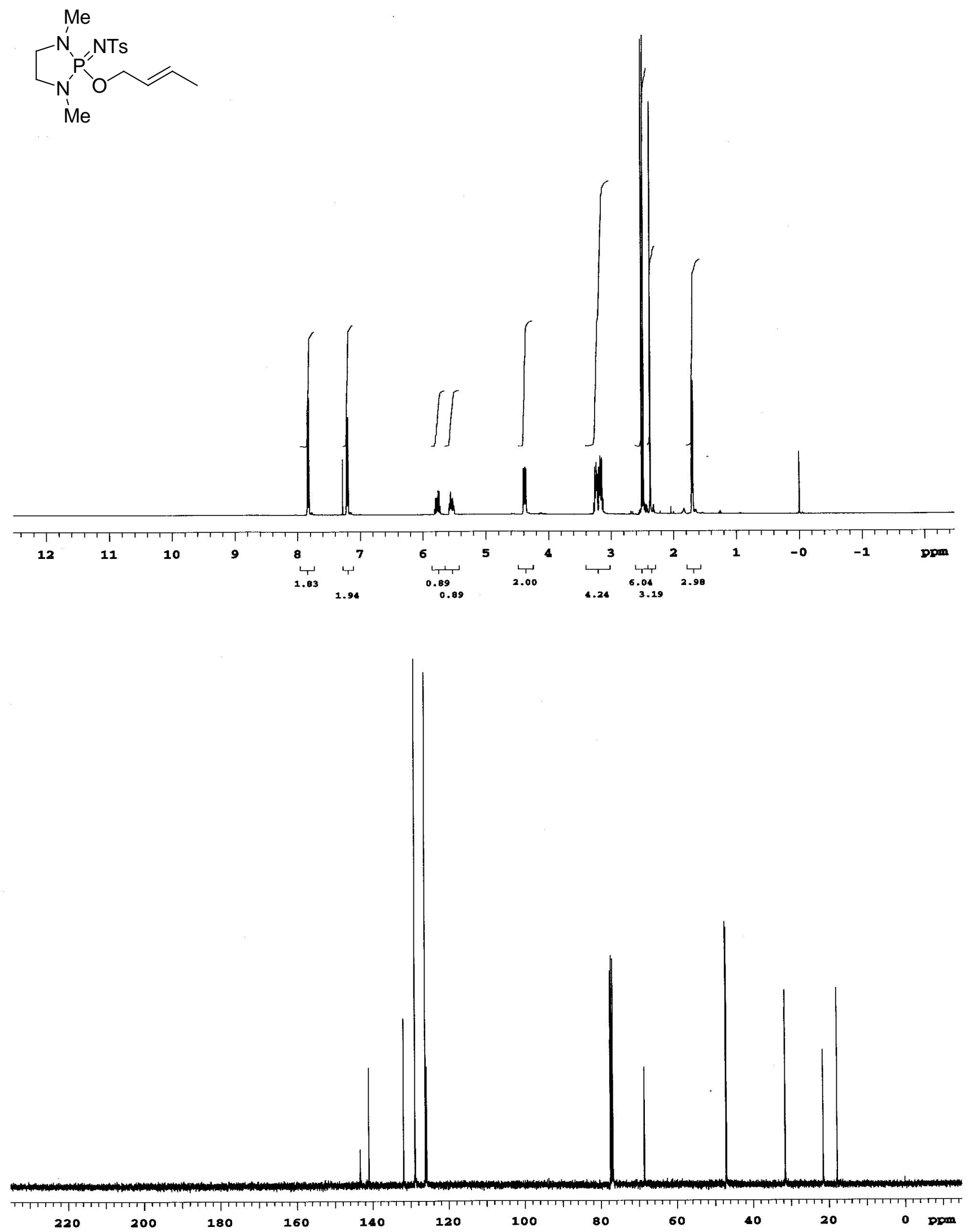

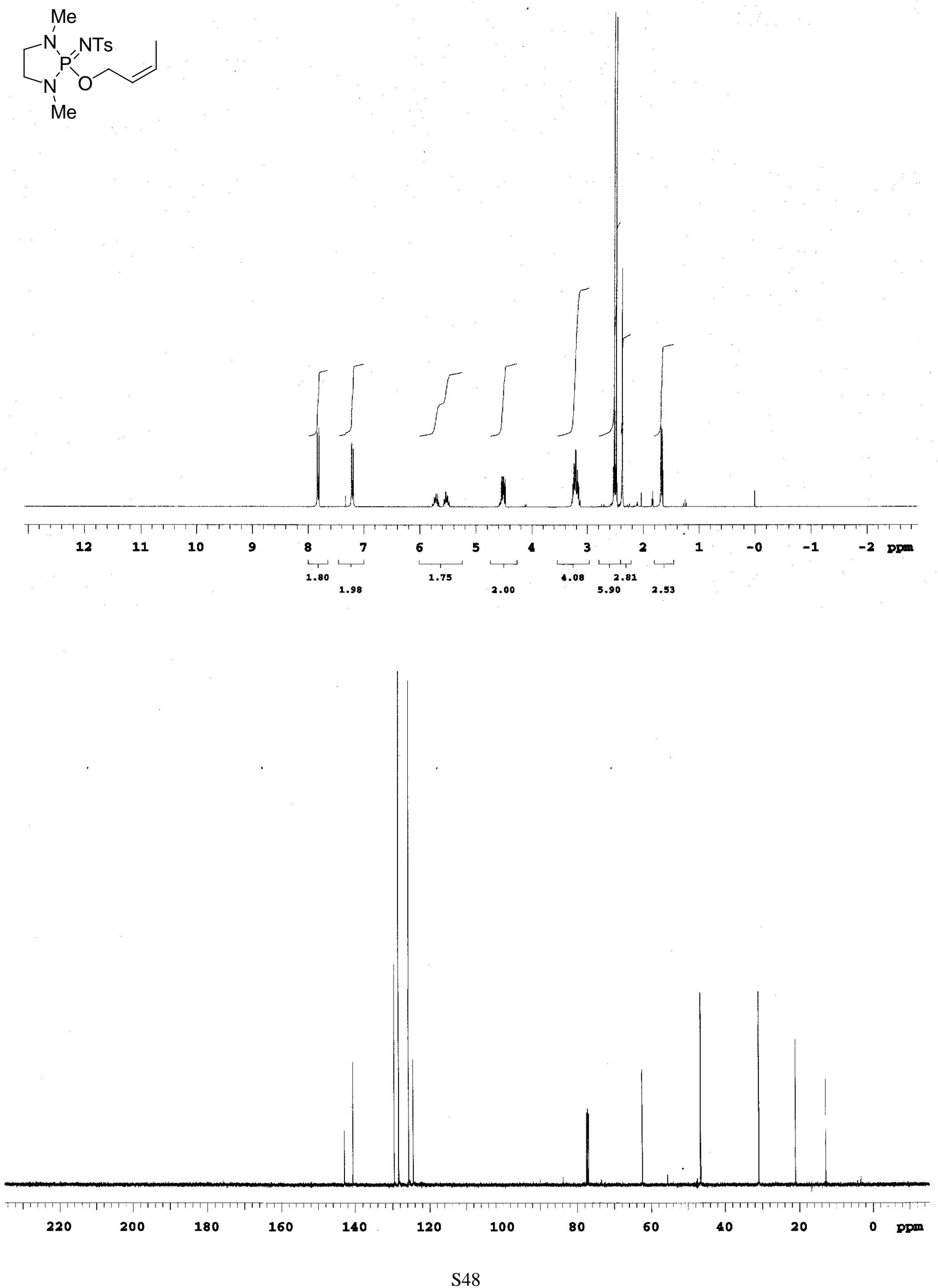
Me
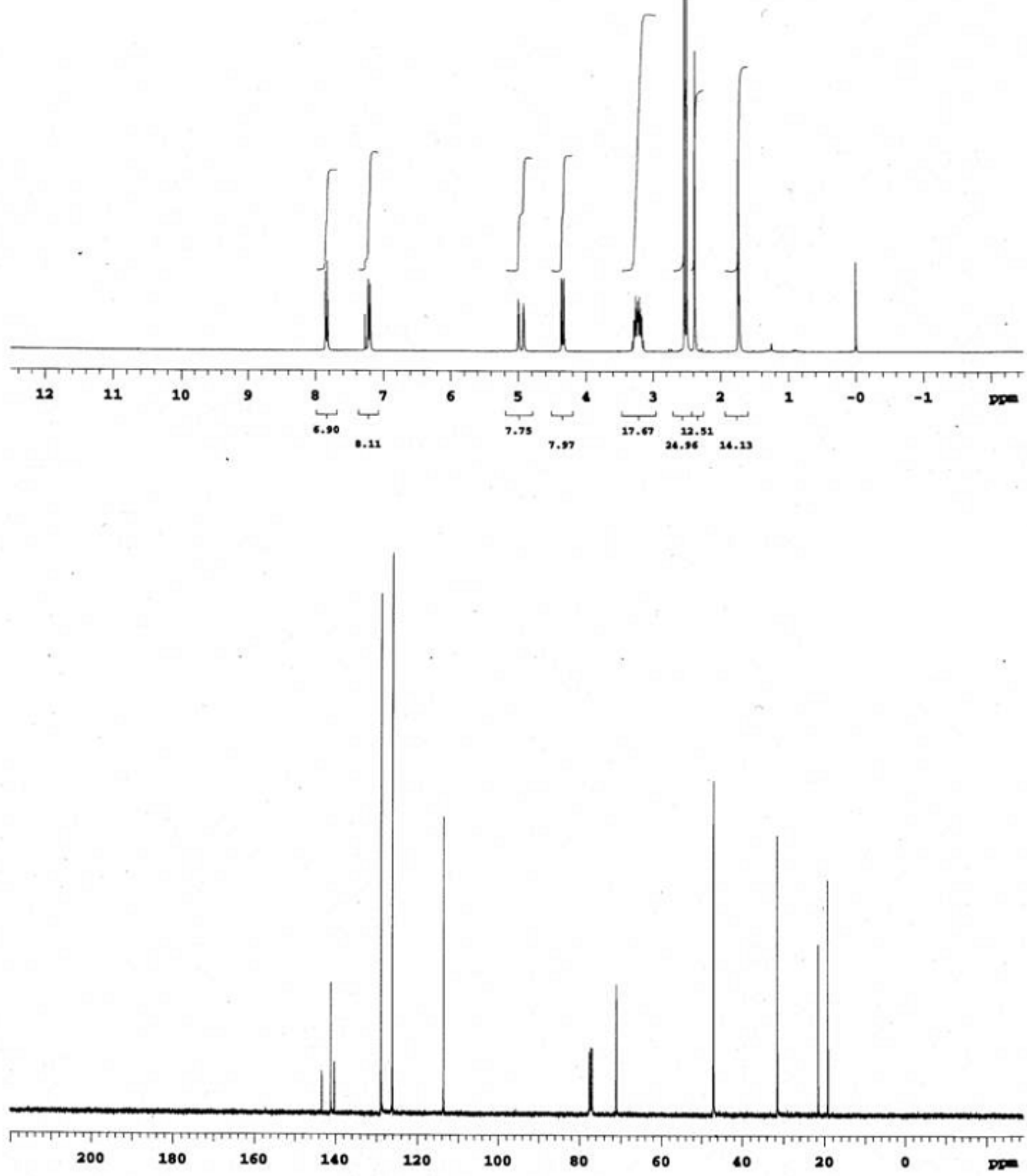


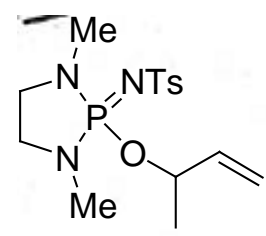
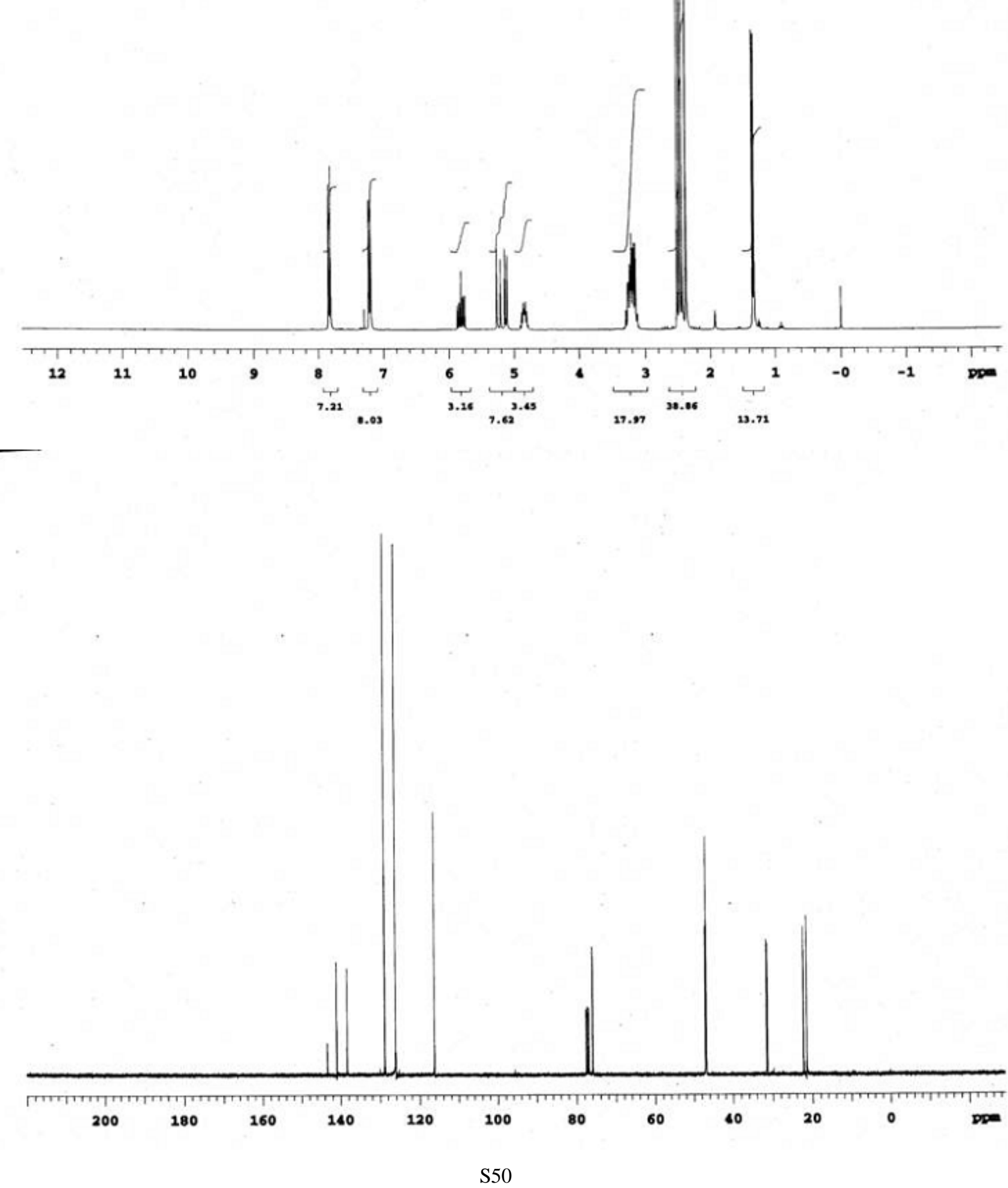

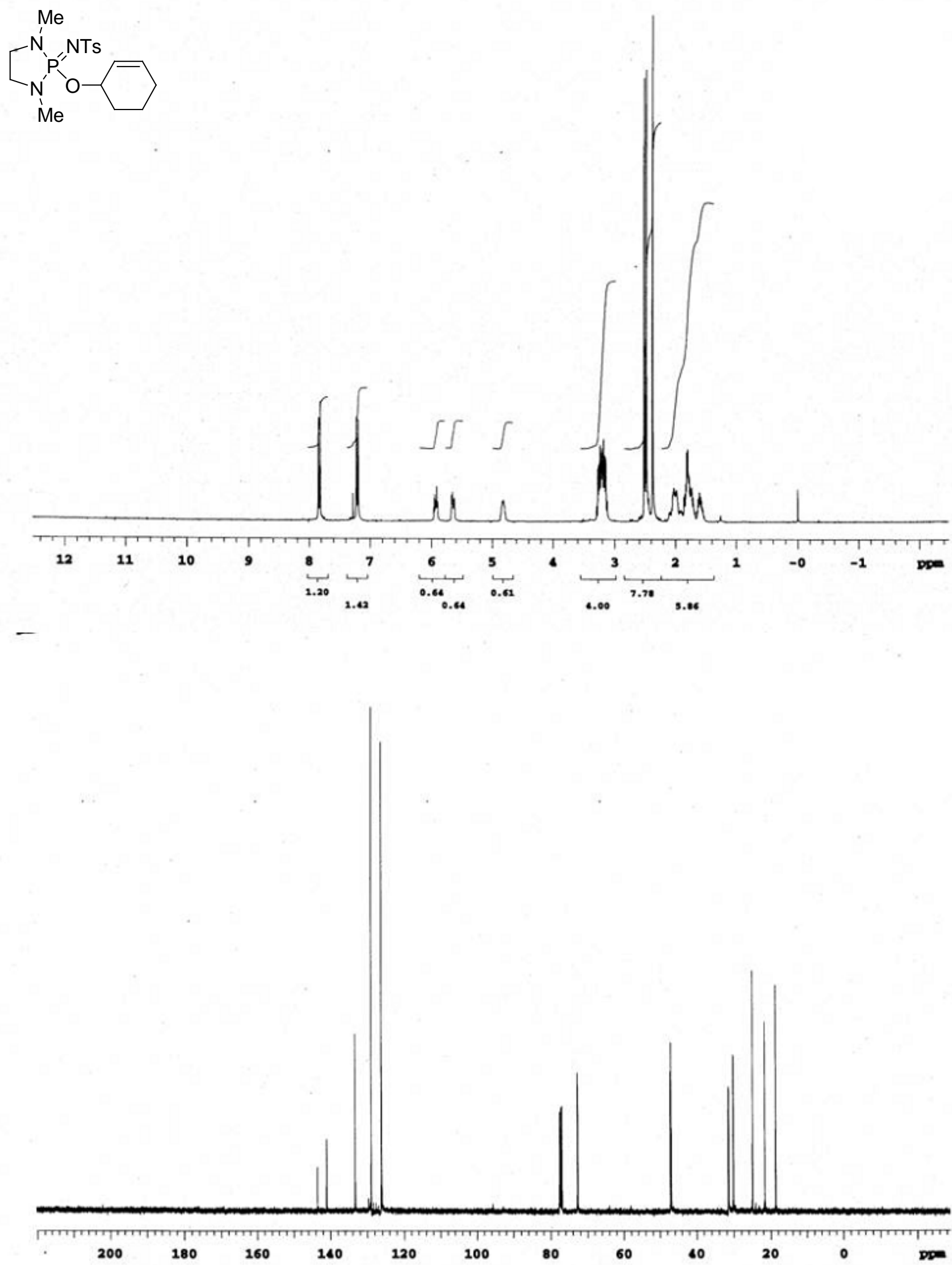

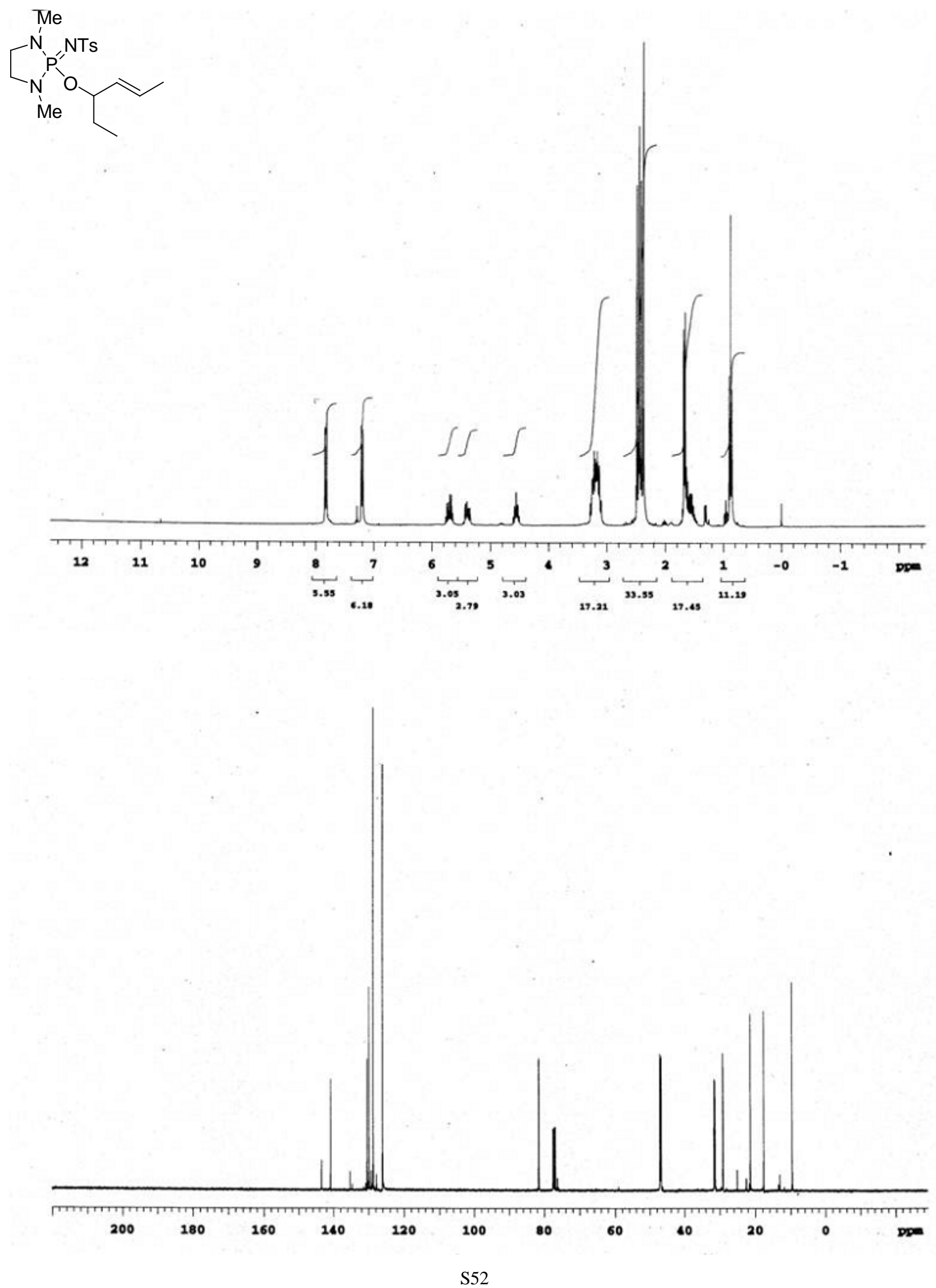

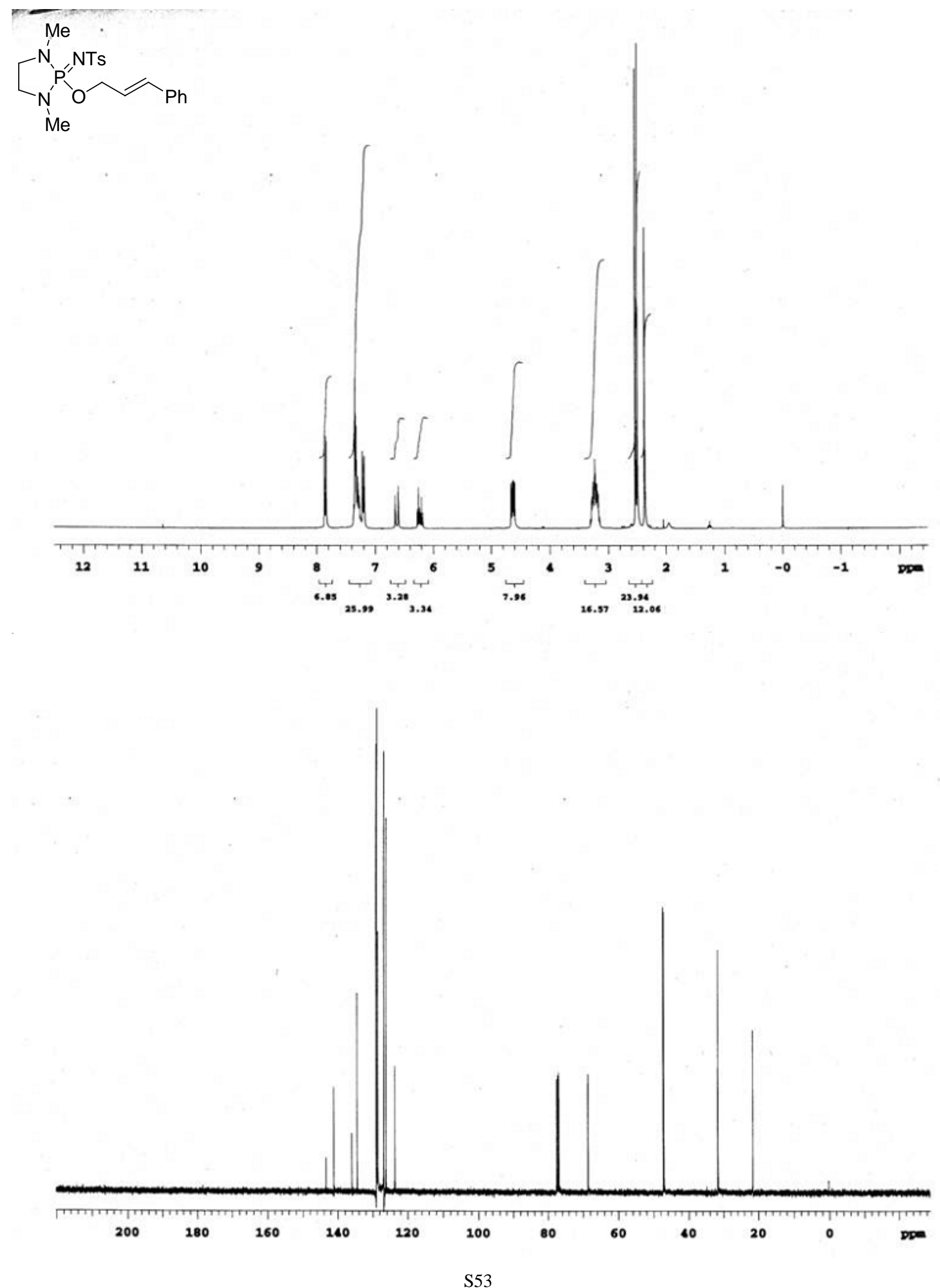

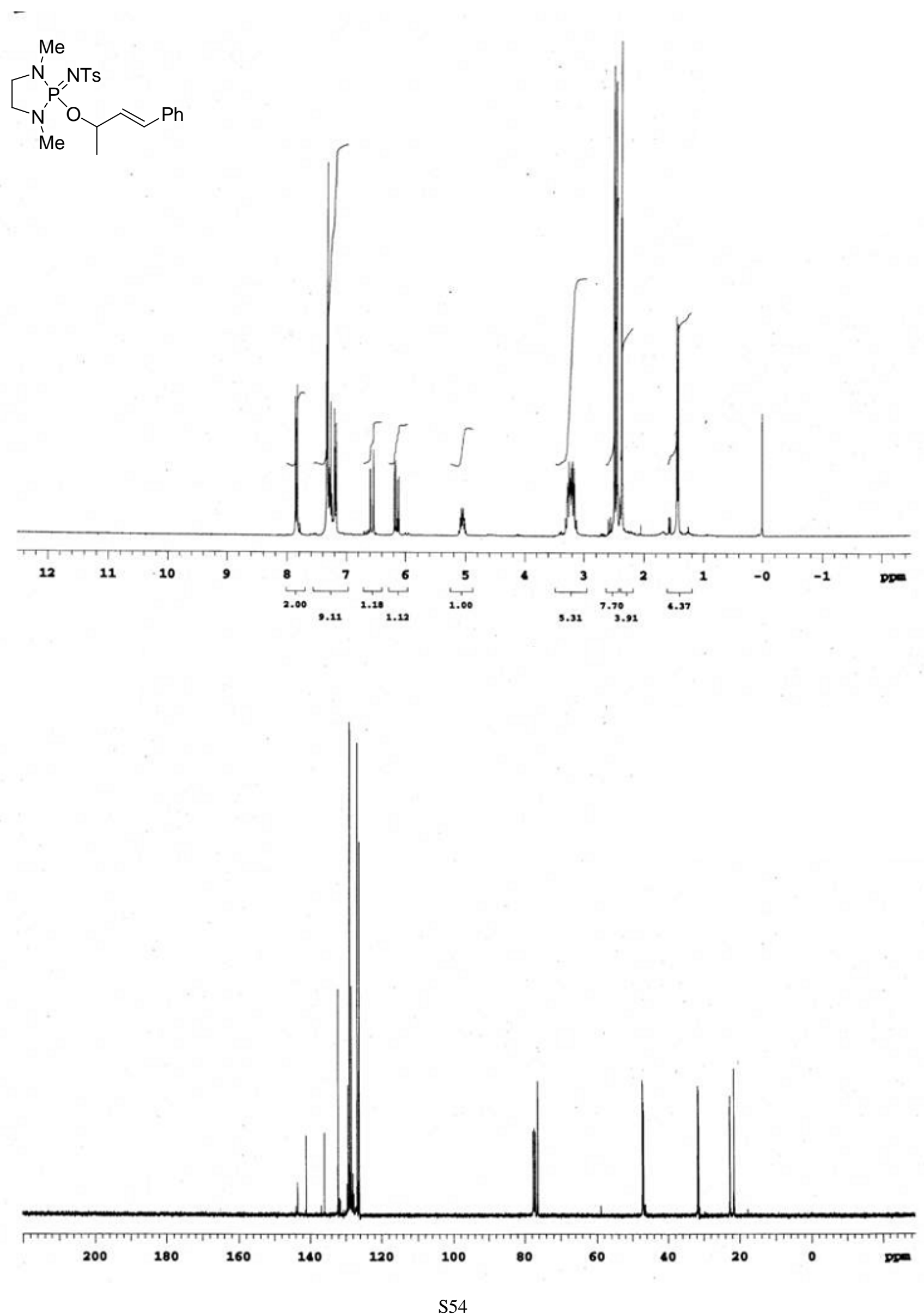

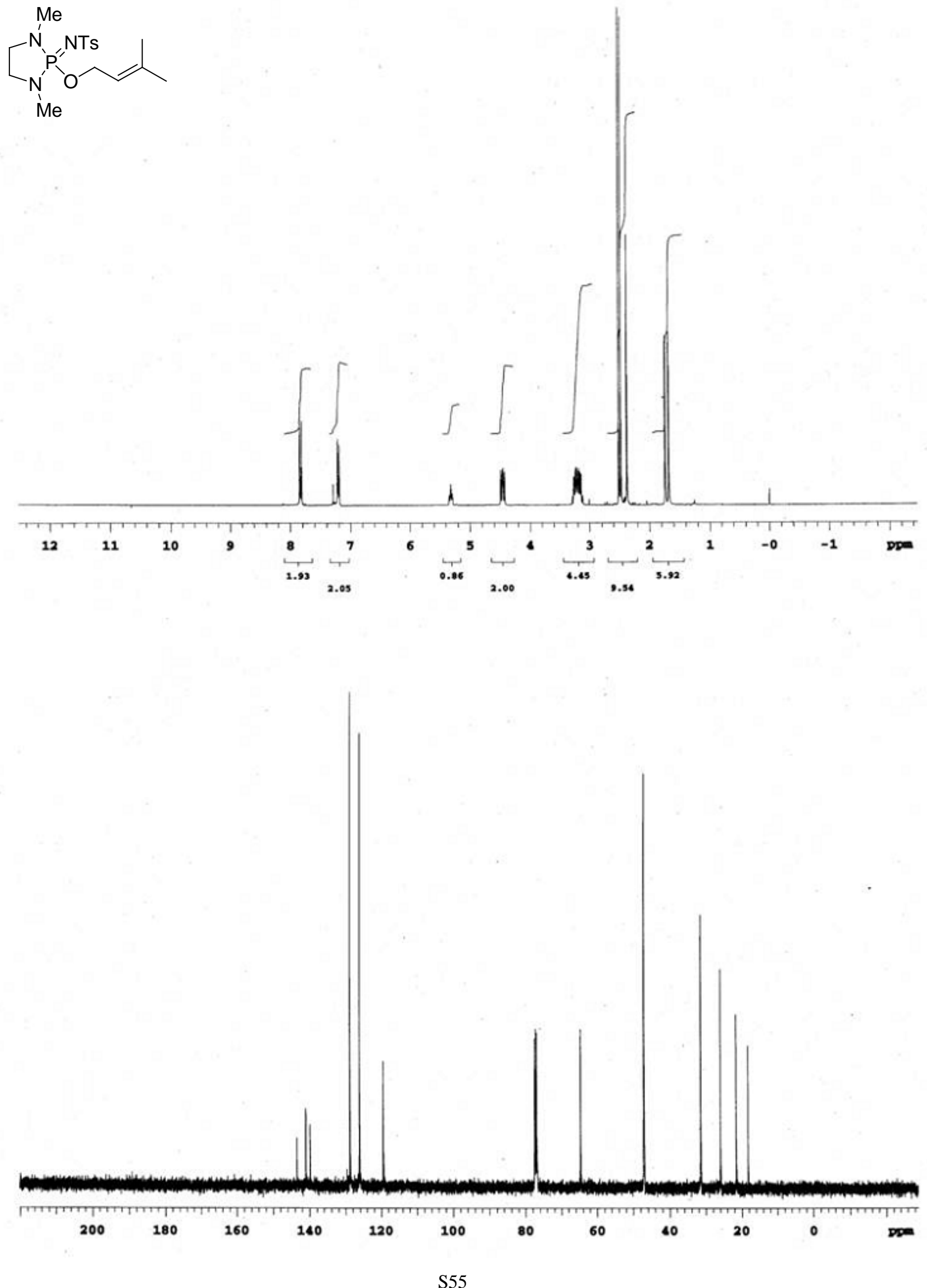

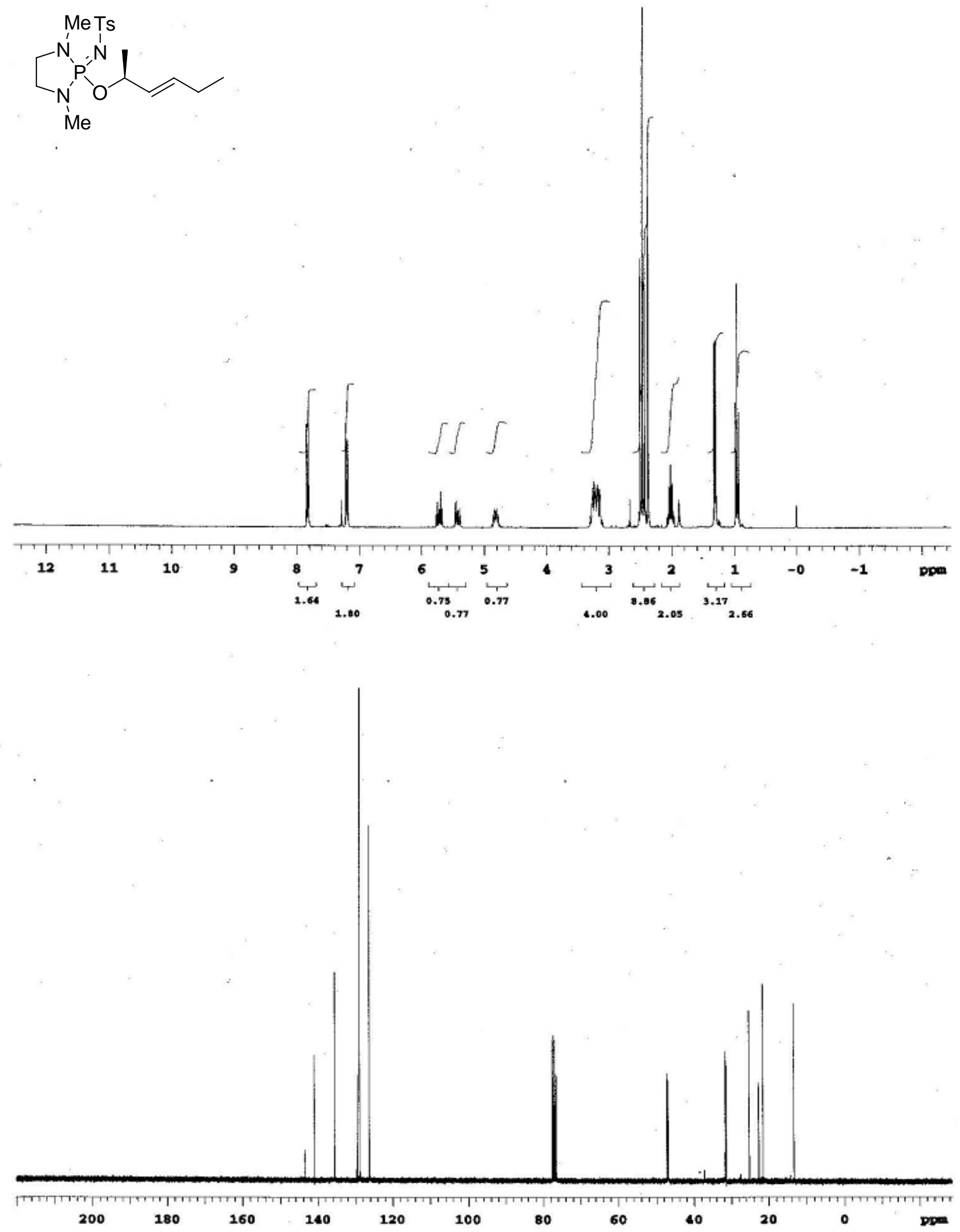

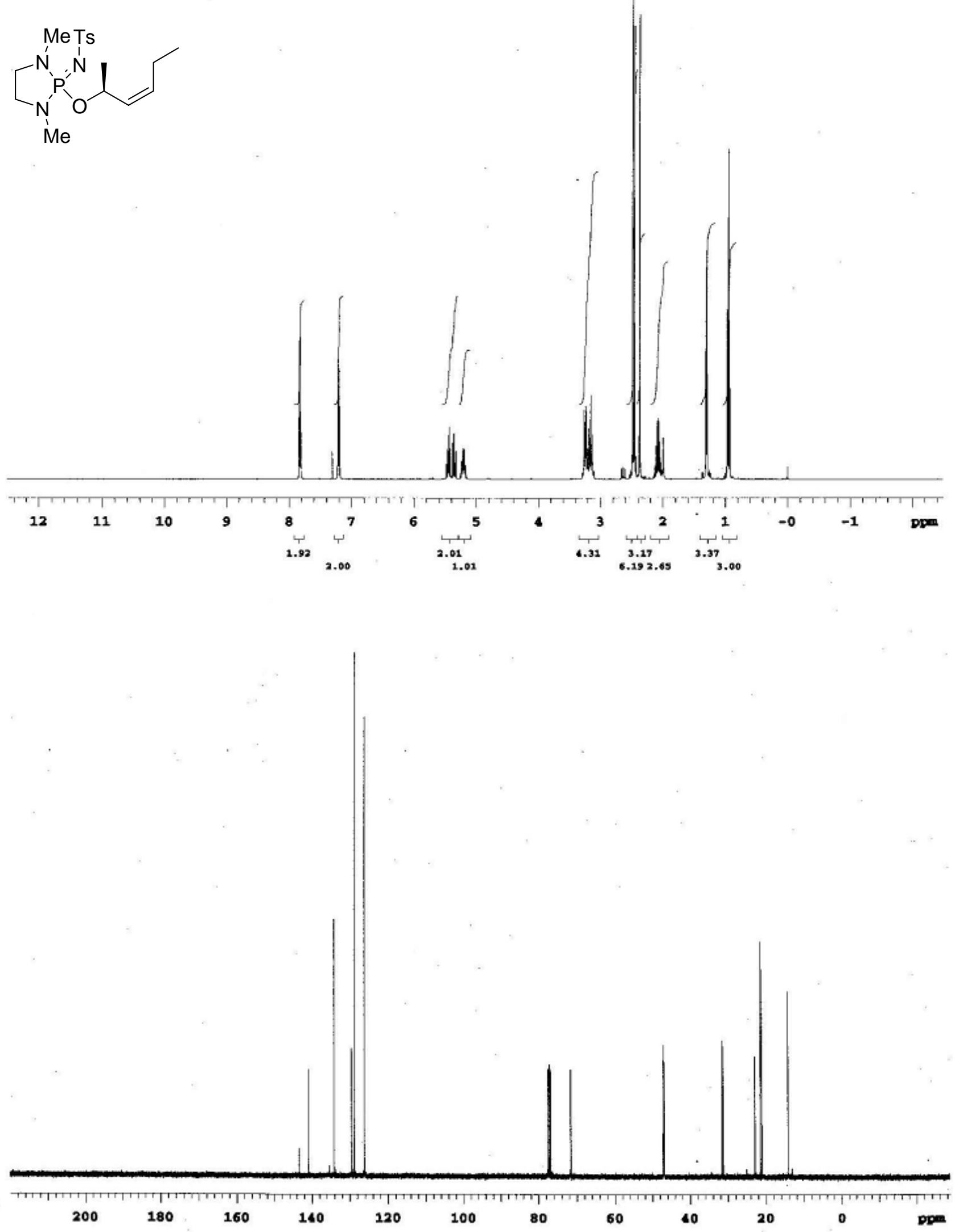

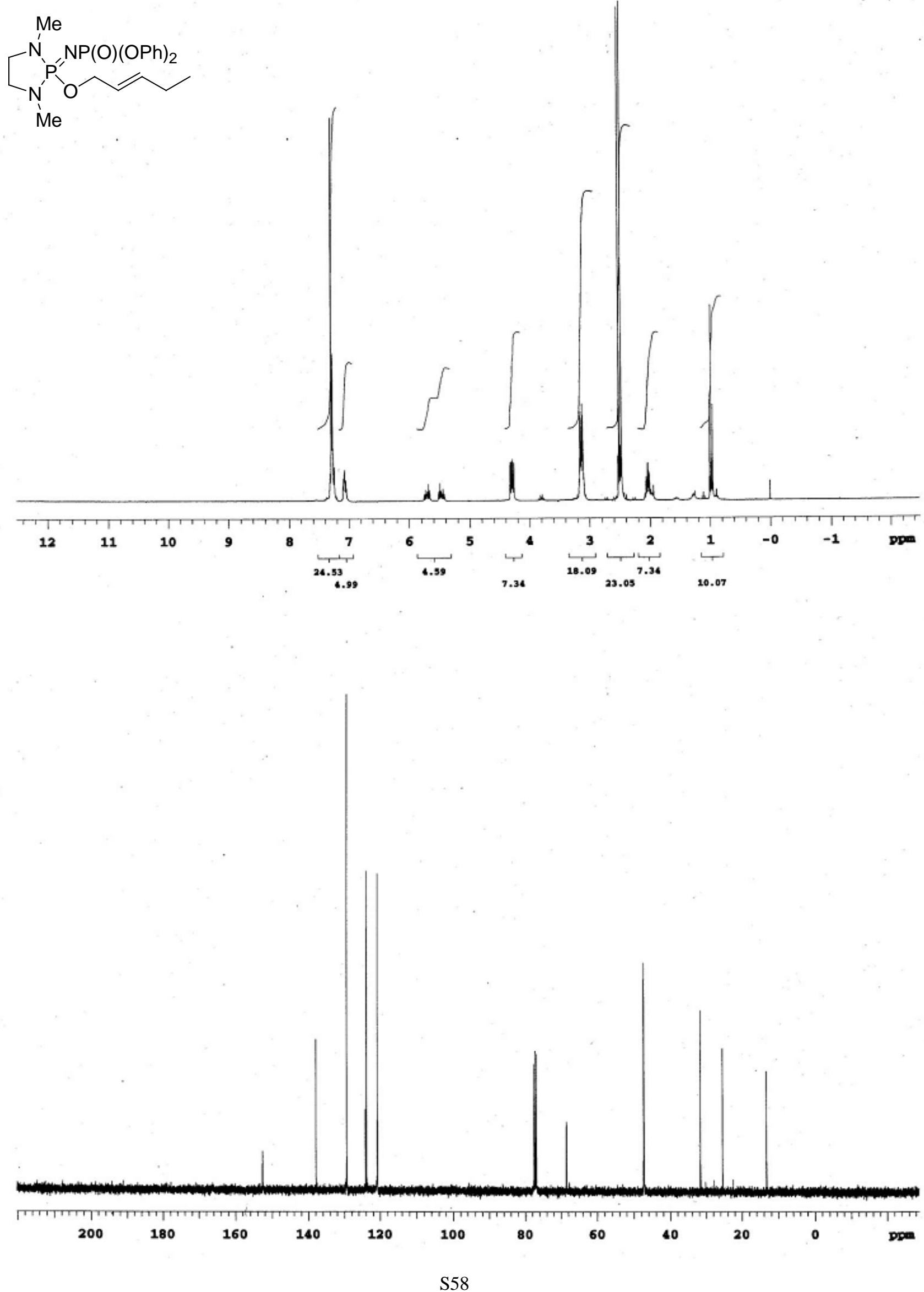

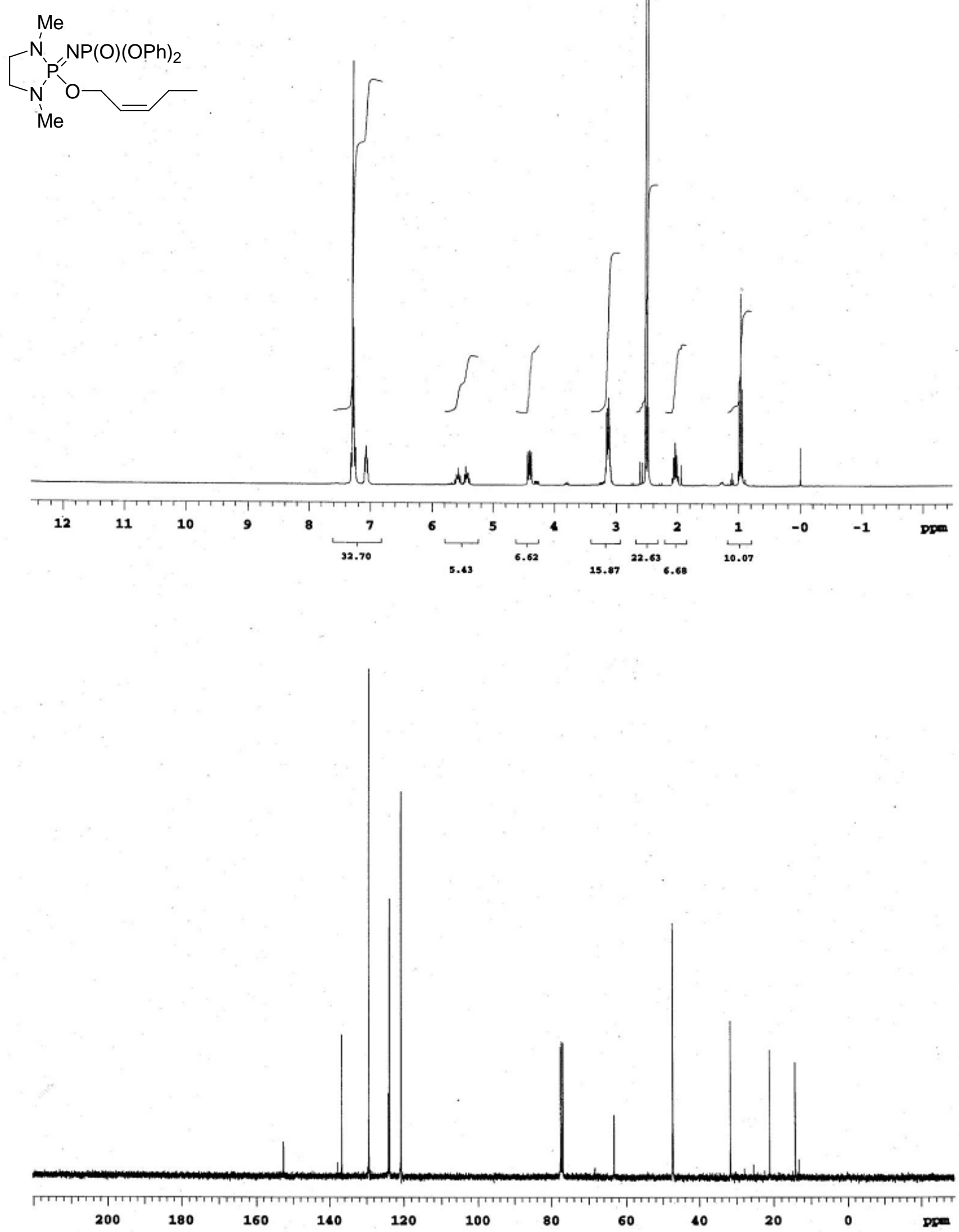


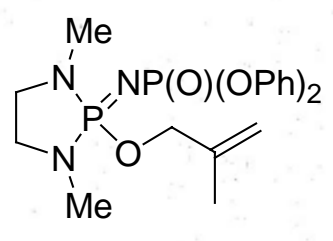
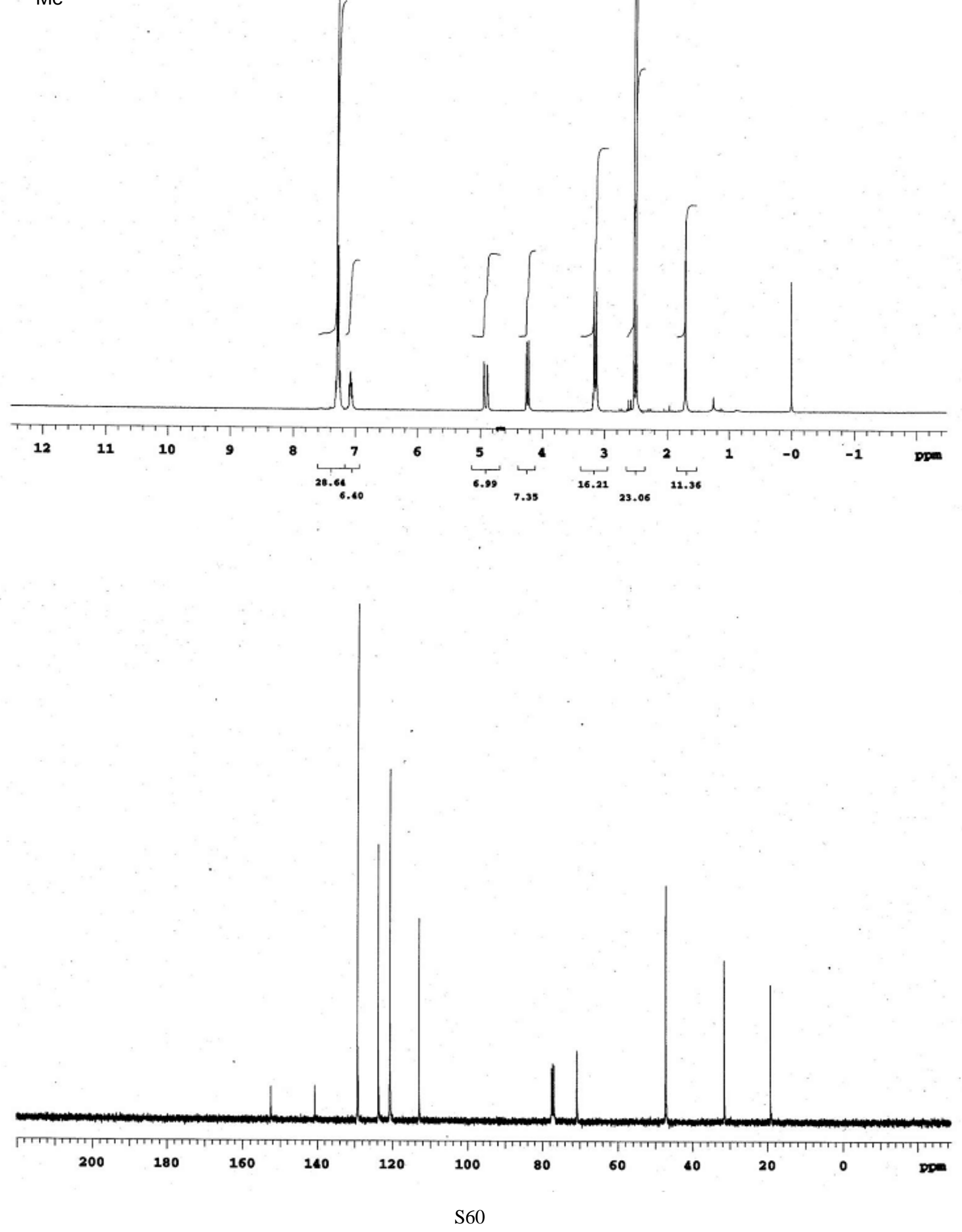

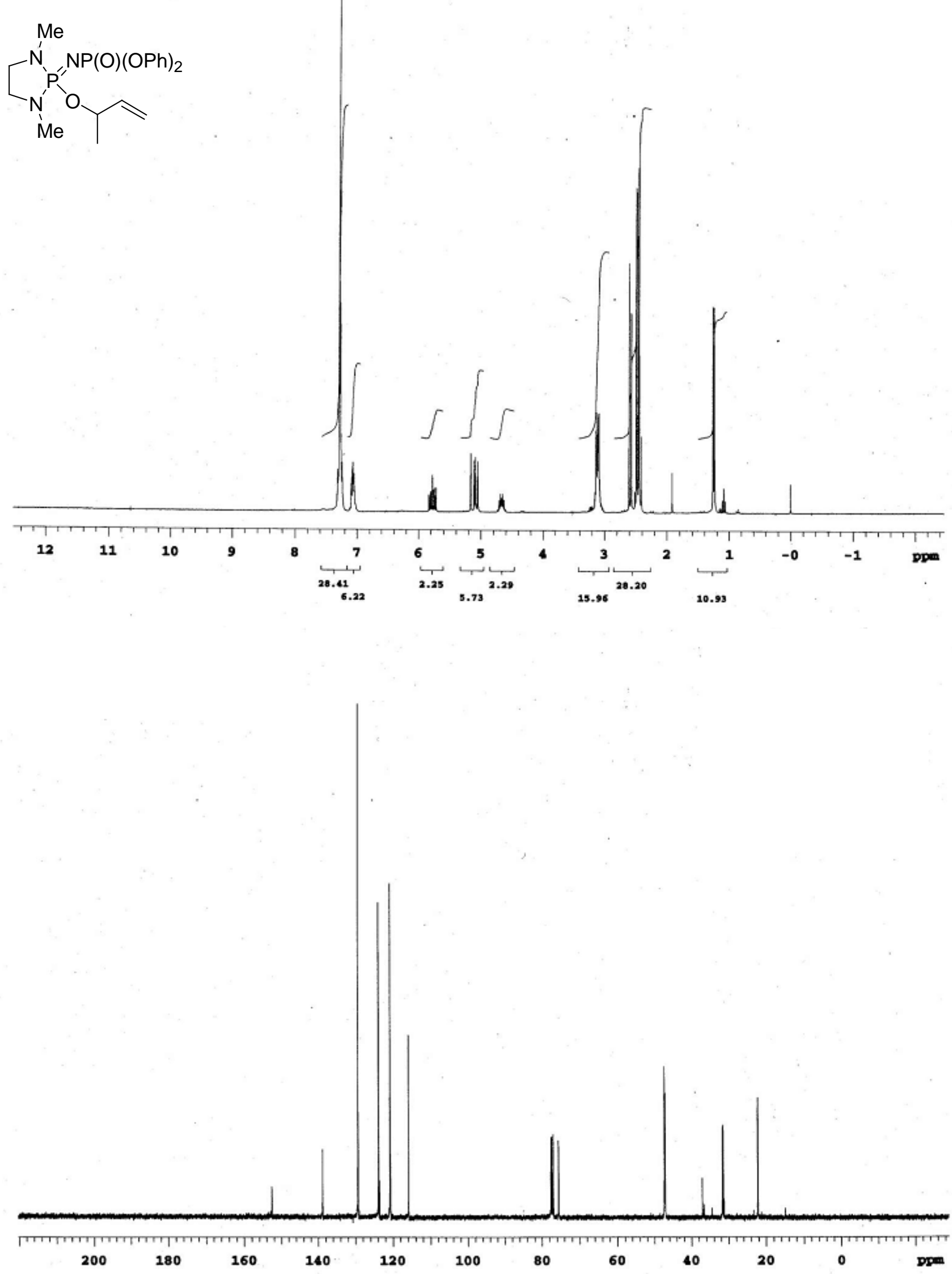

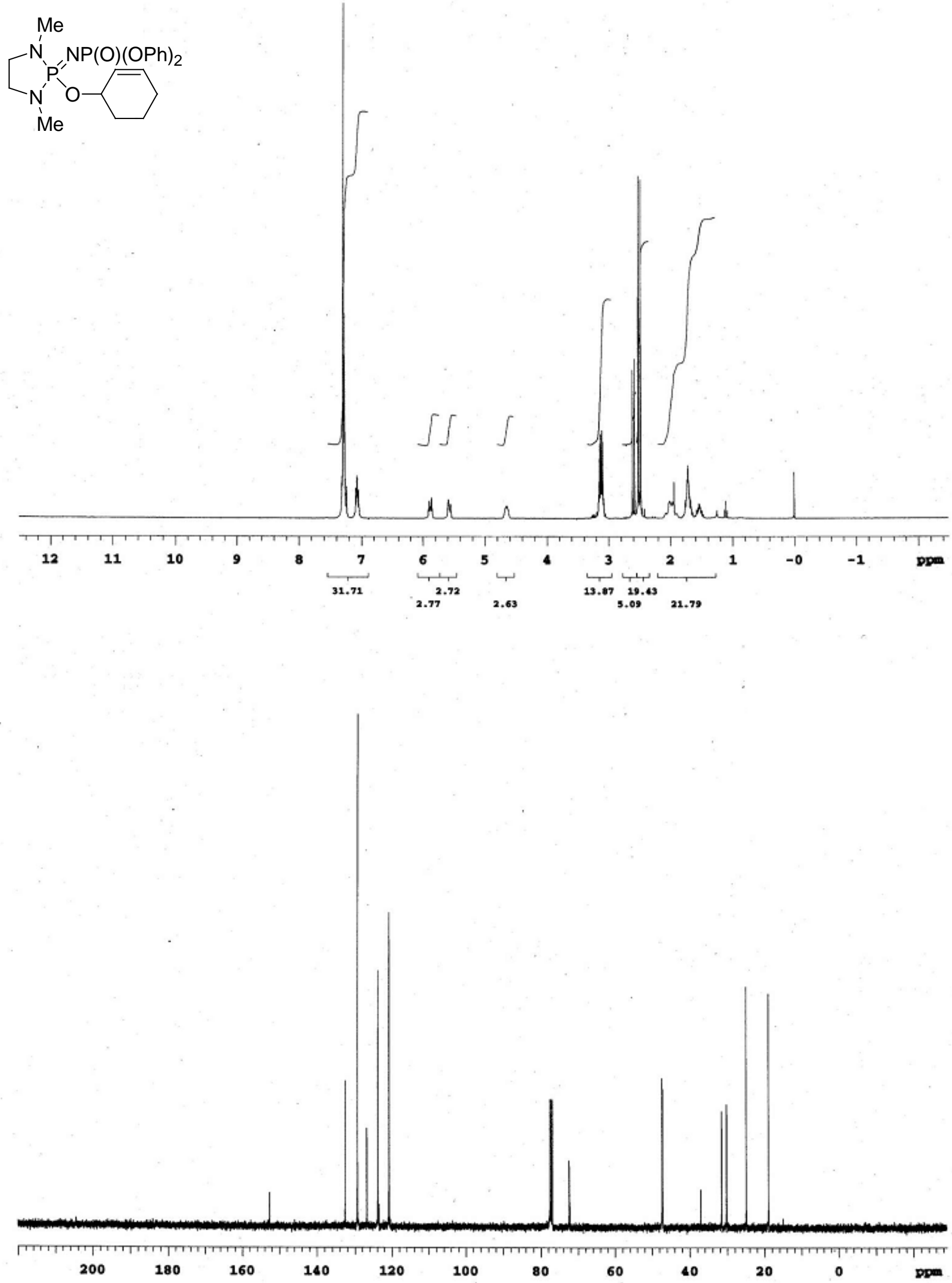

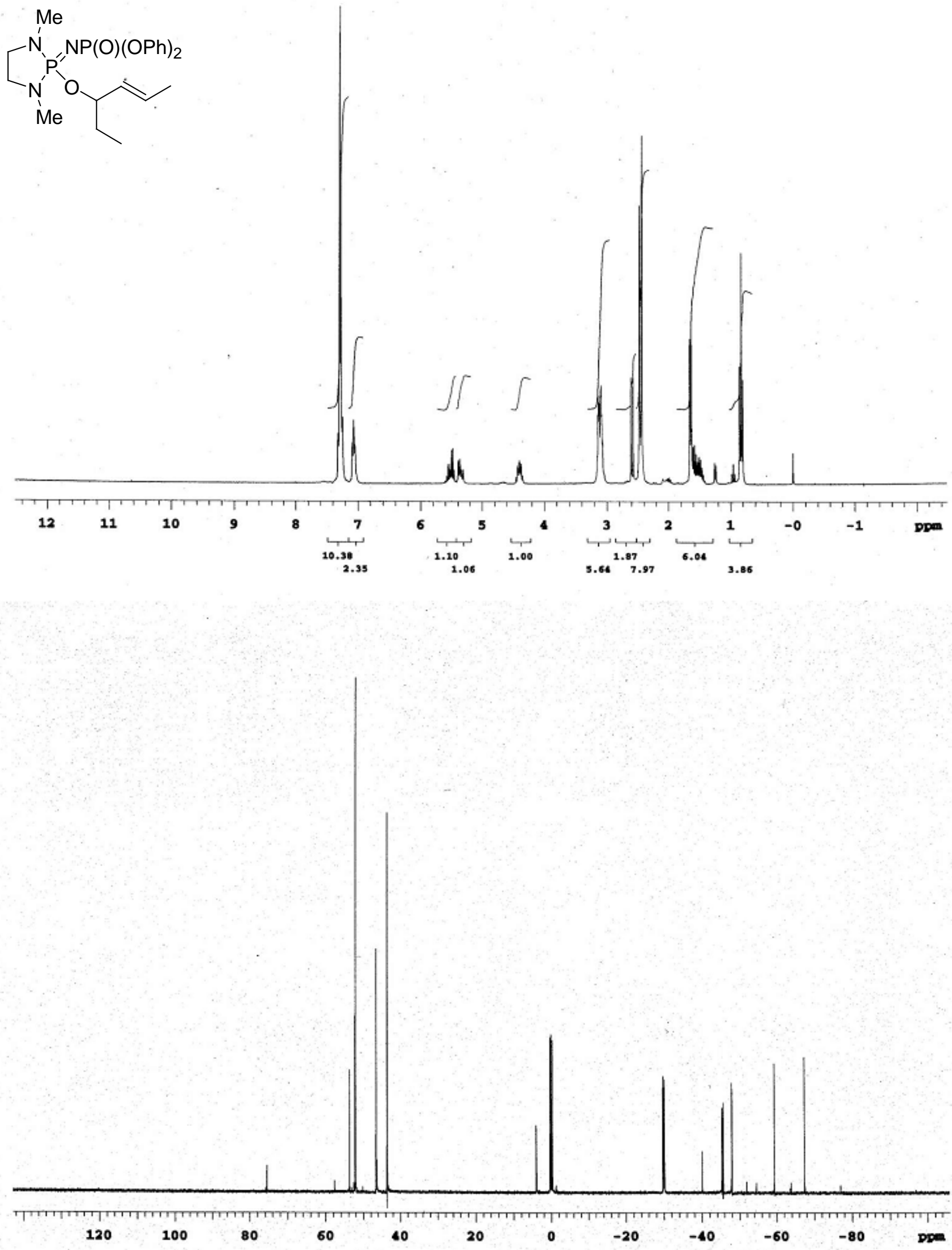

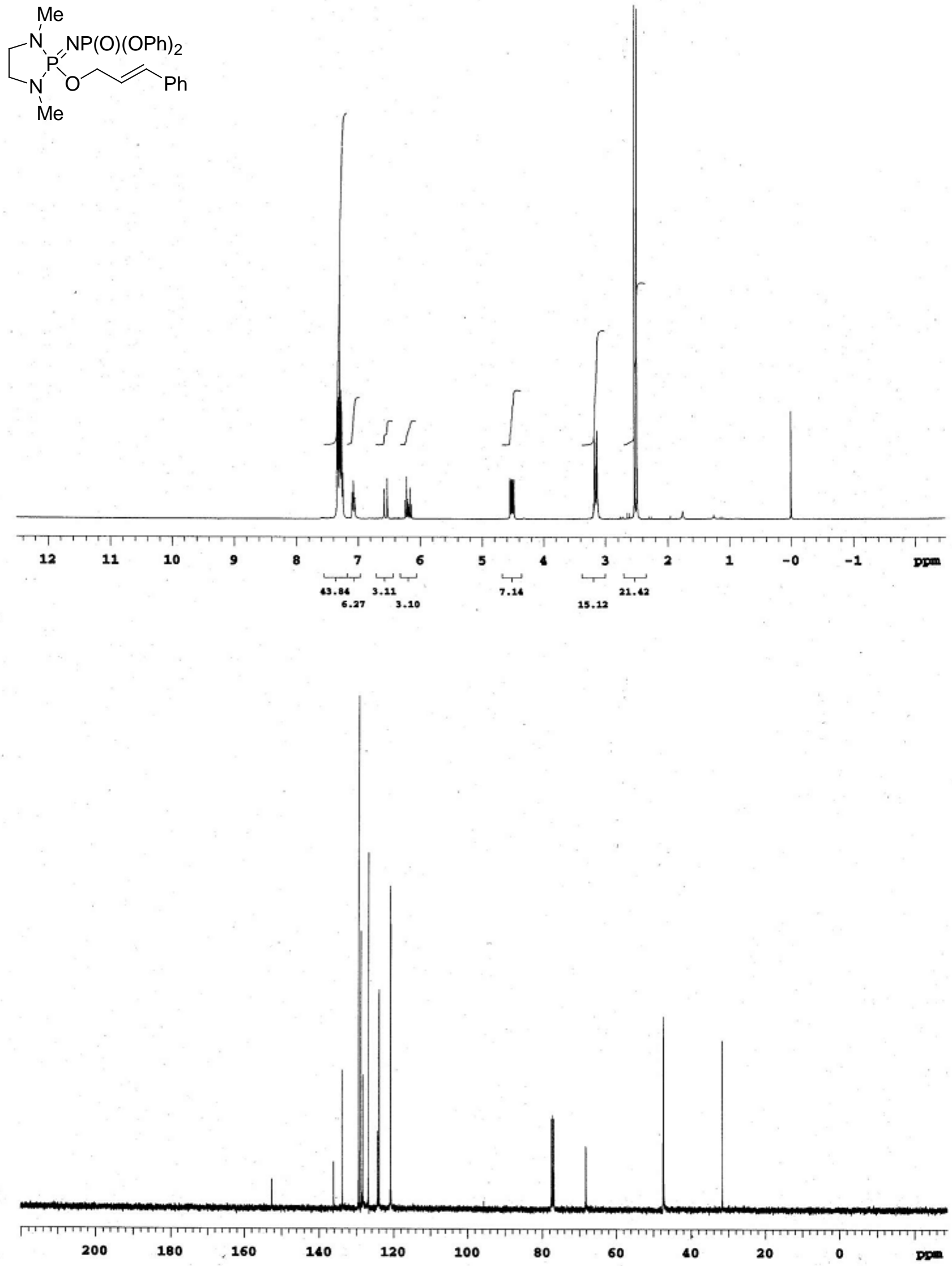

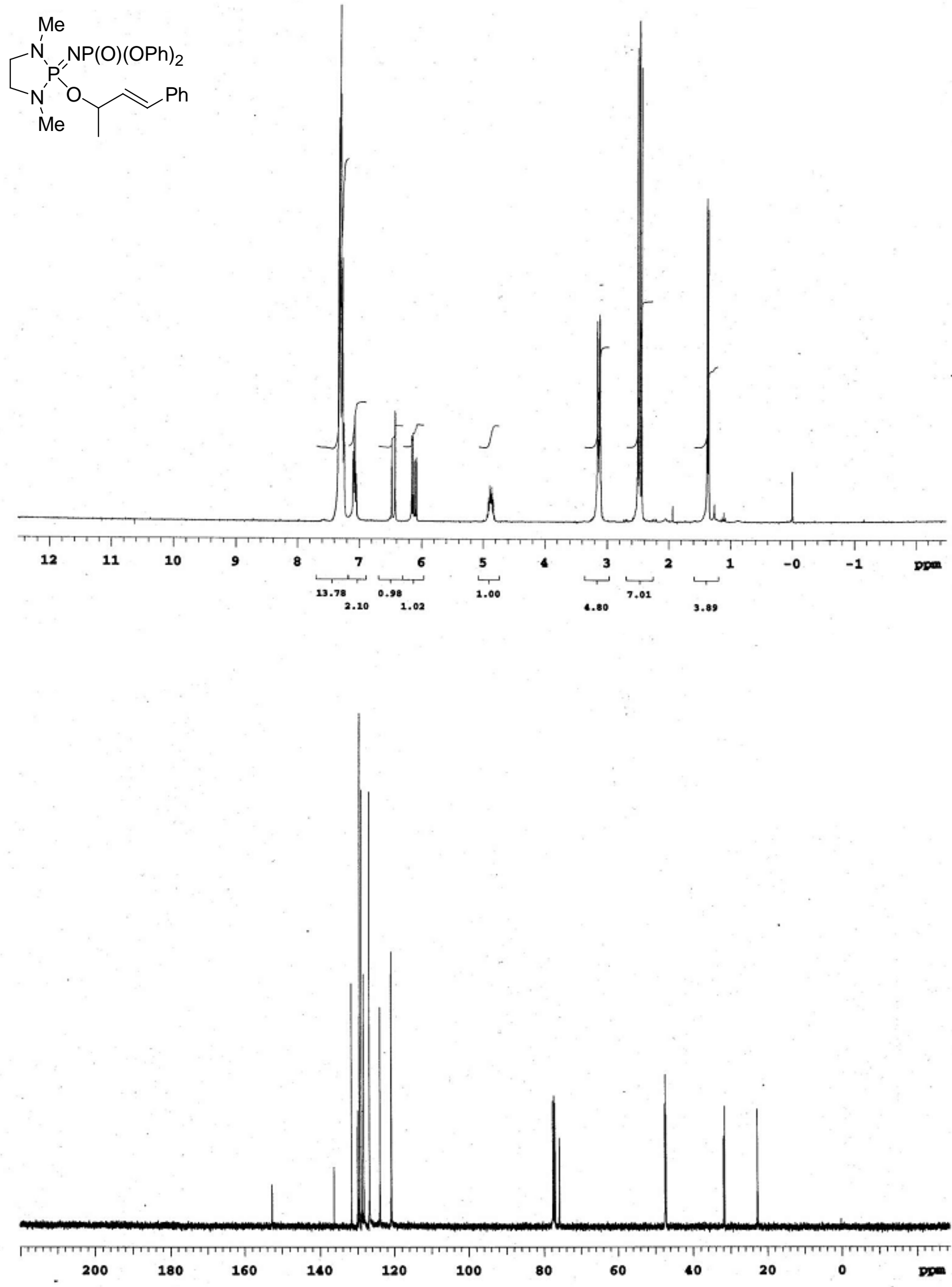

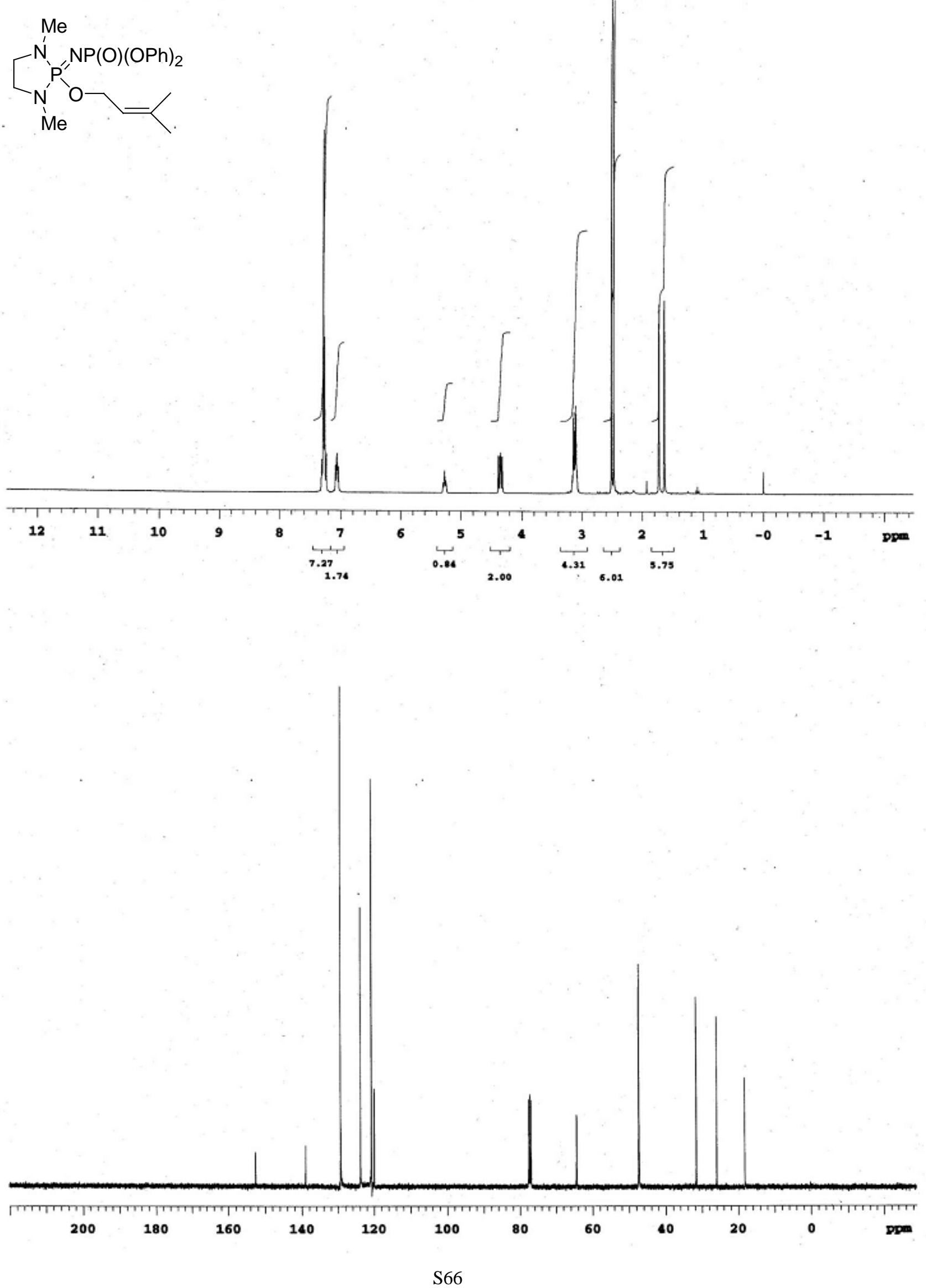

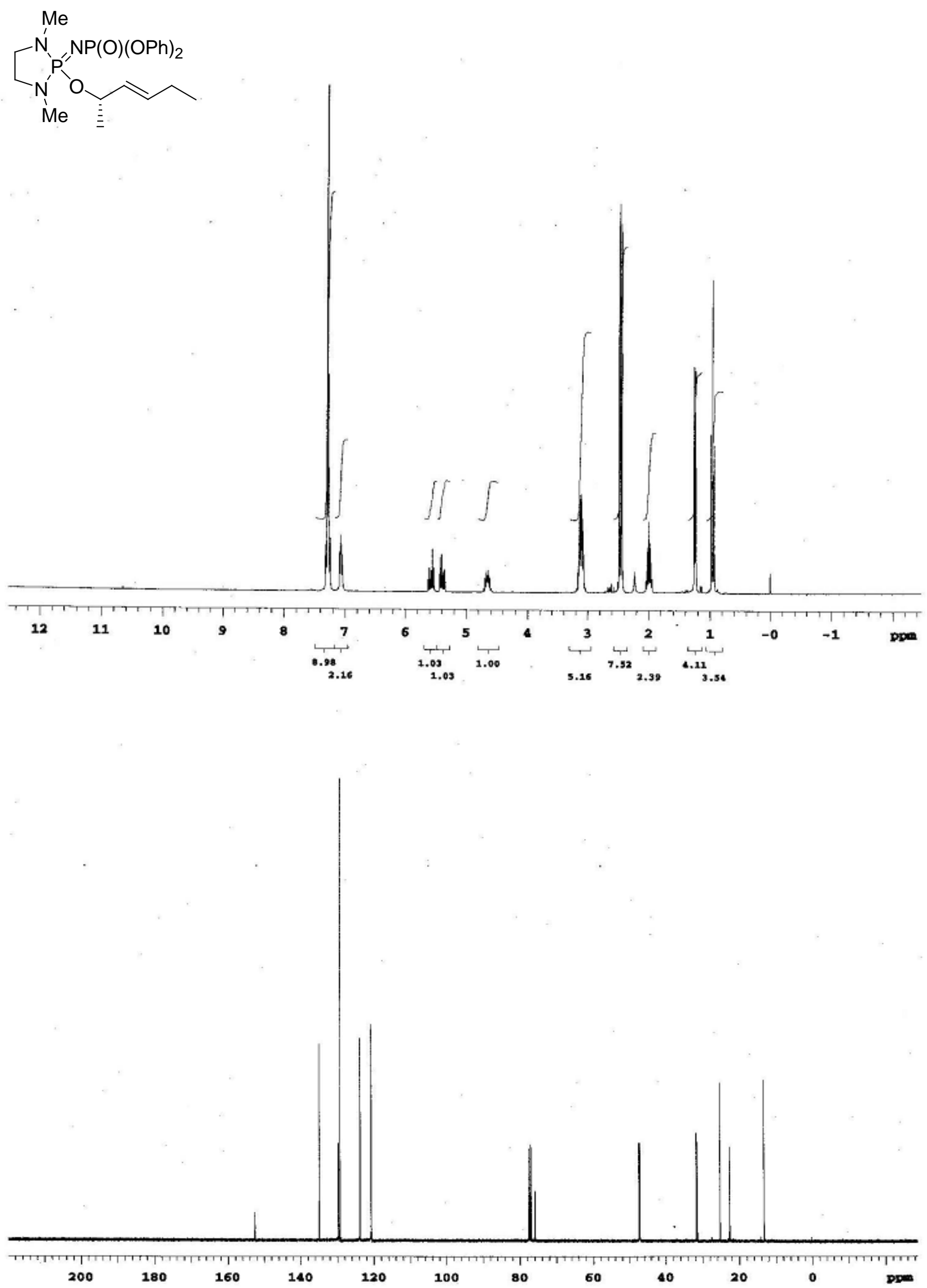
<smiles></smiles>
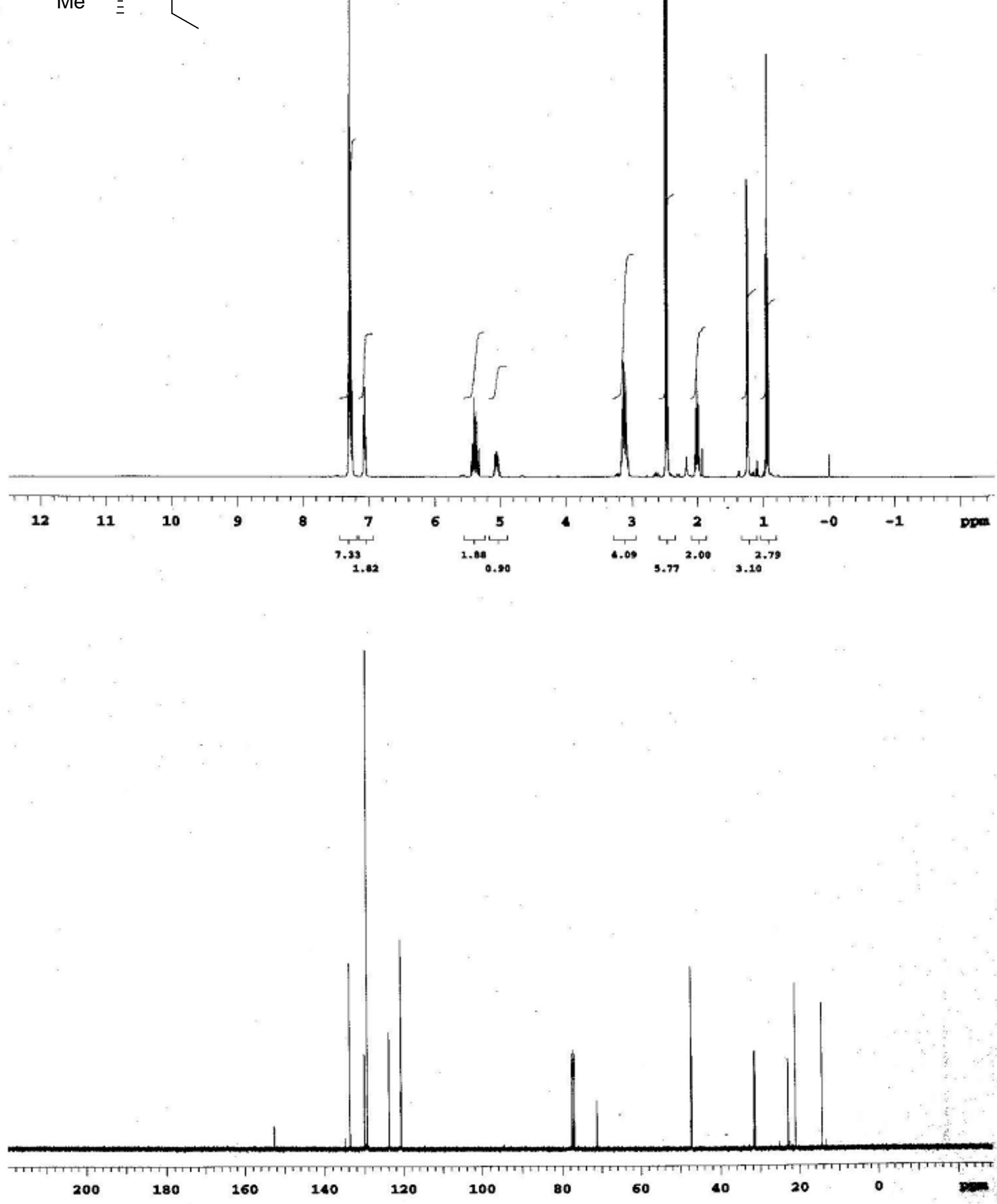

S68 

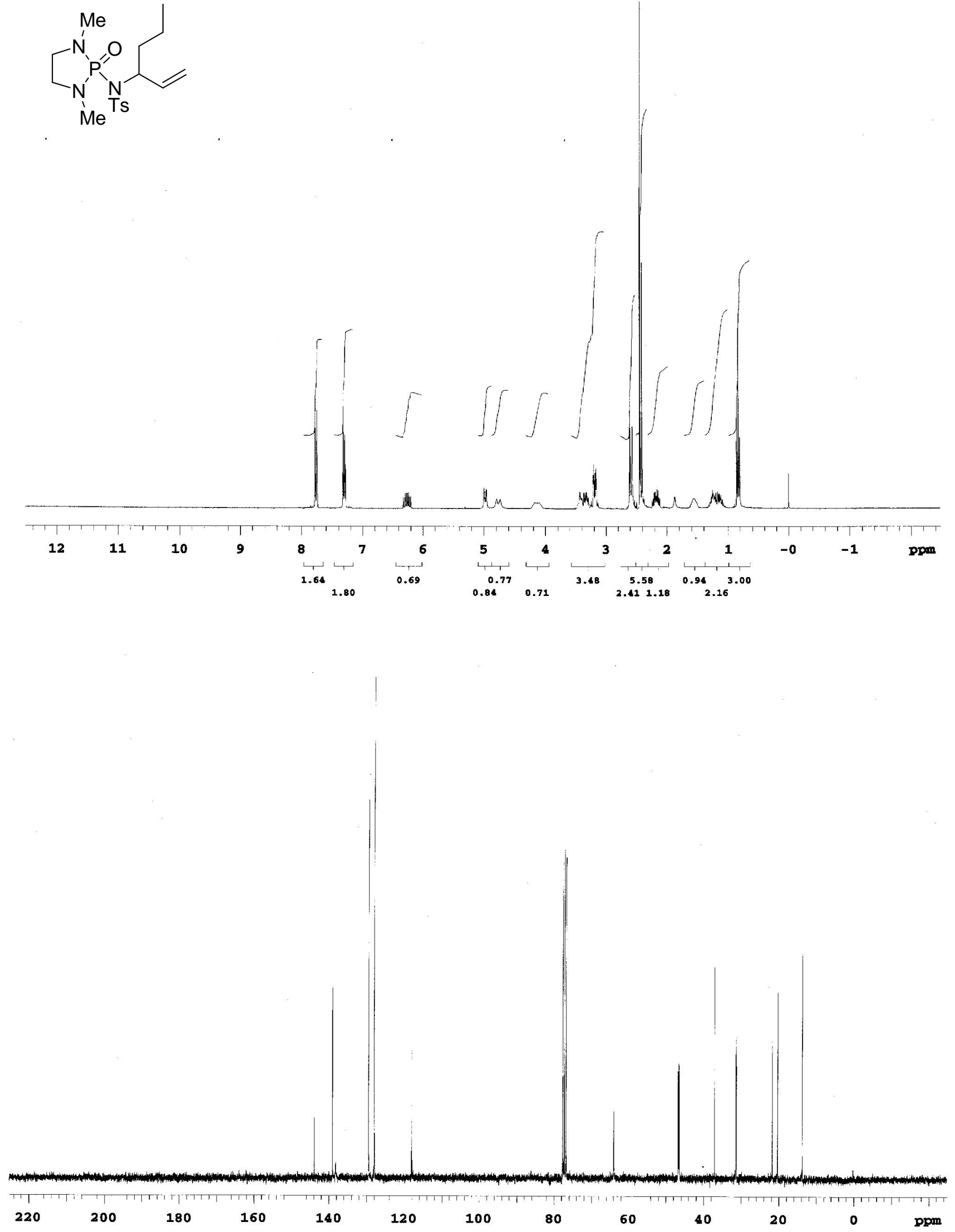
<smiles>C=CC(CC)N(C)P1(=O)N(C)CCN1C</smiles>

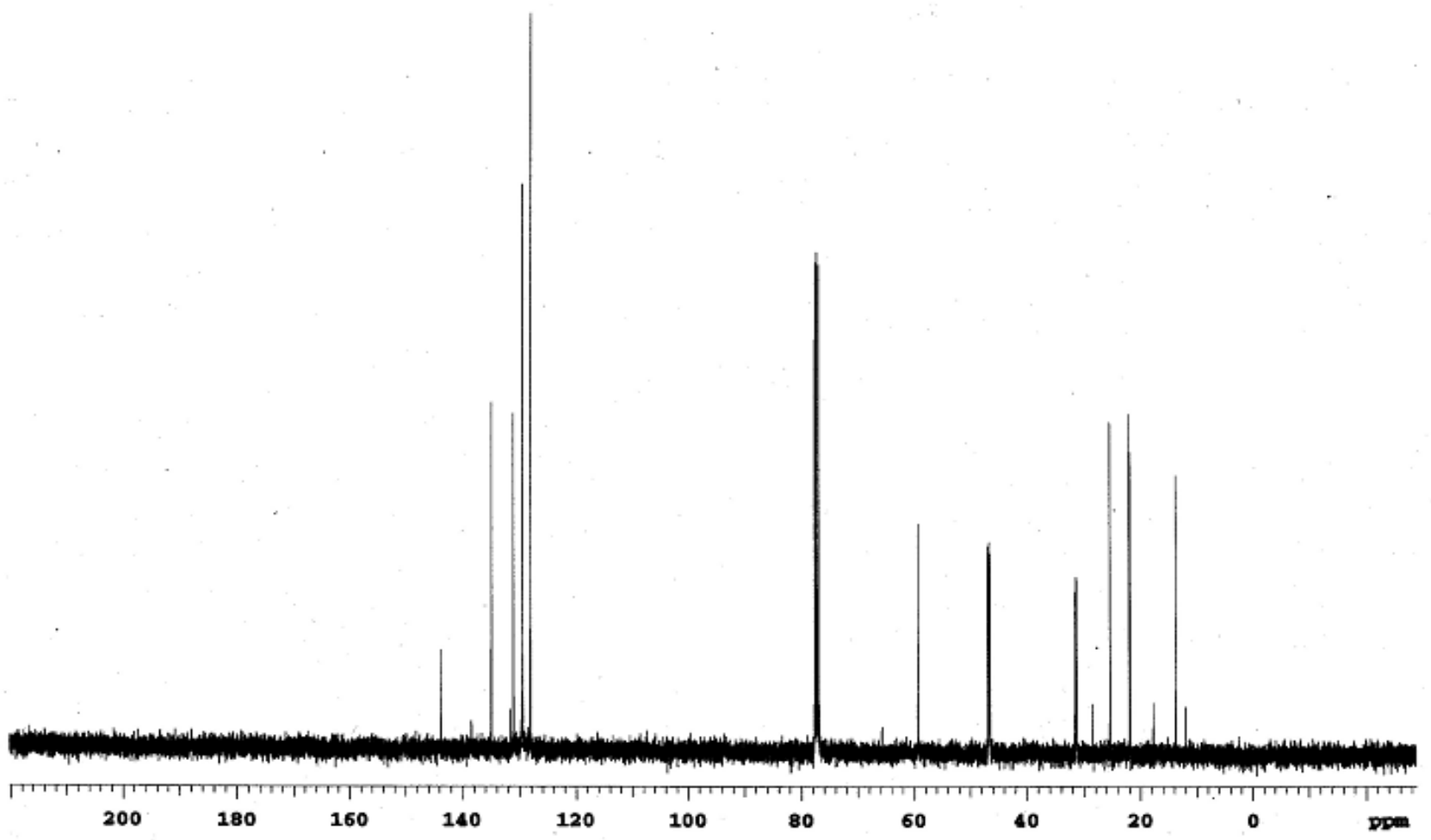



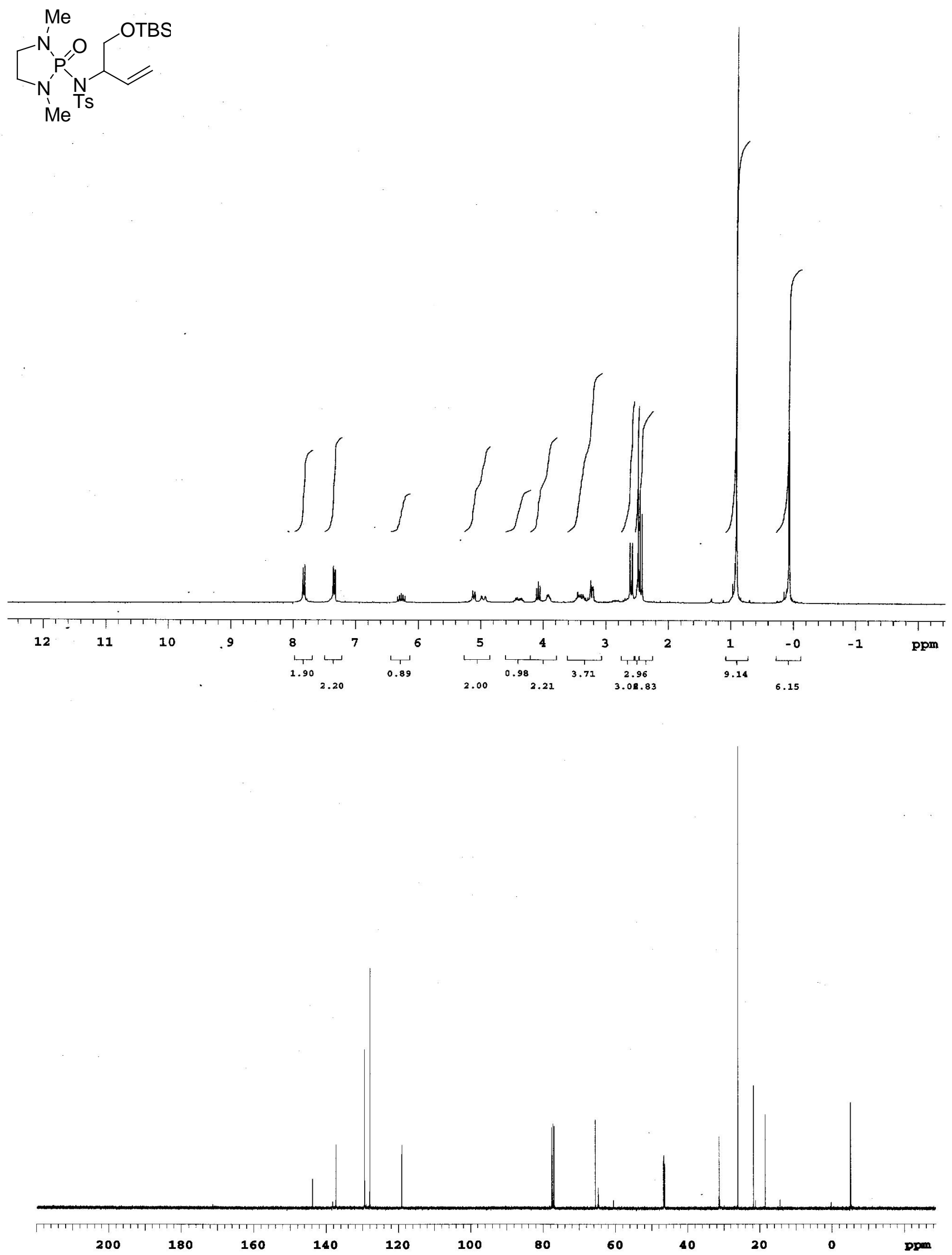

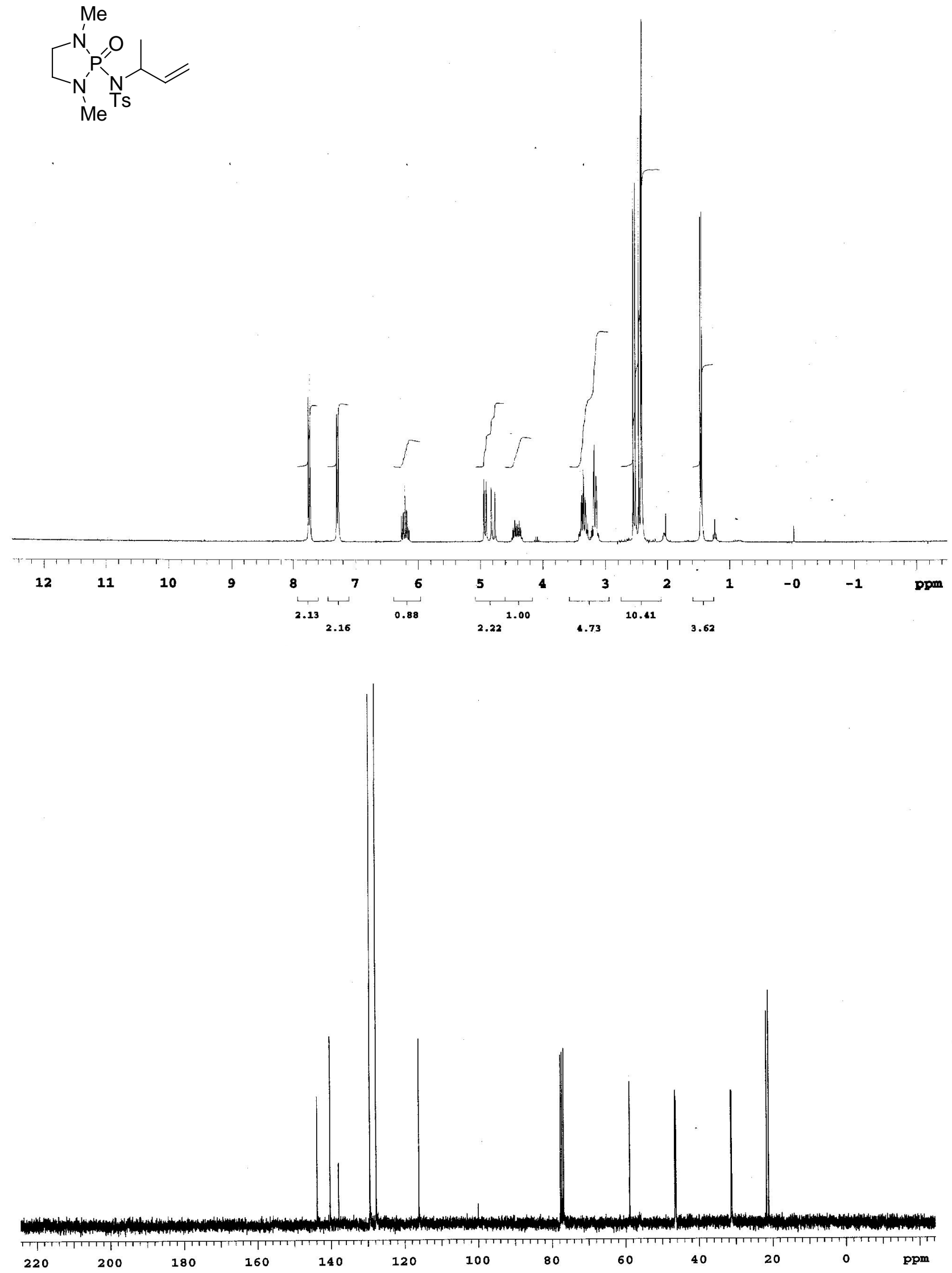
Clse
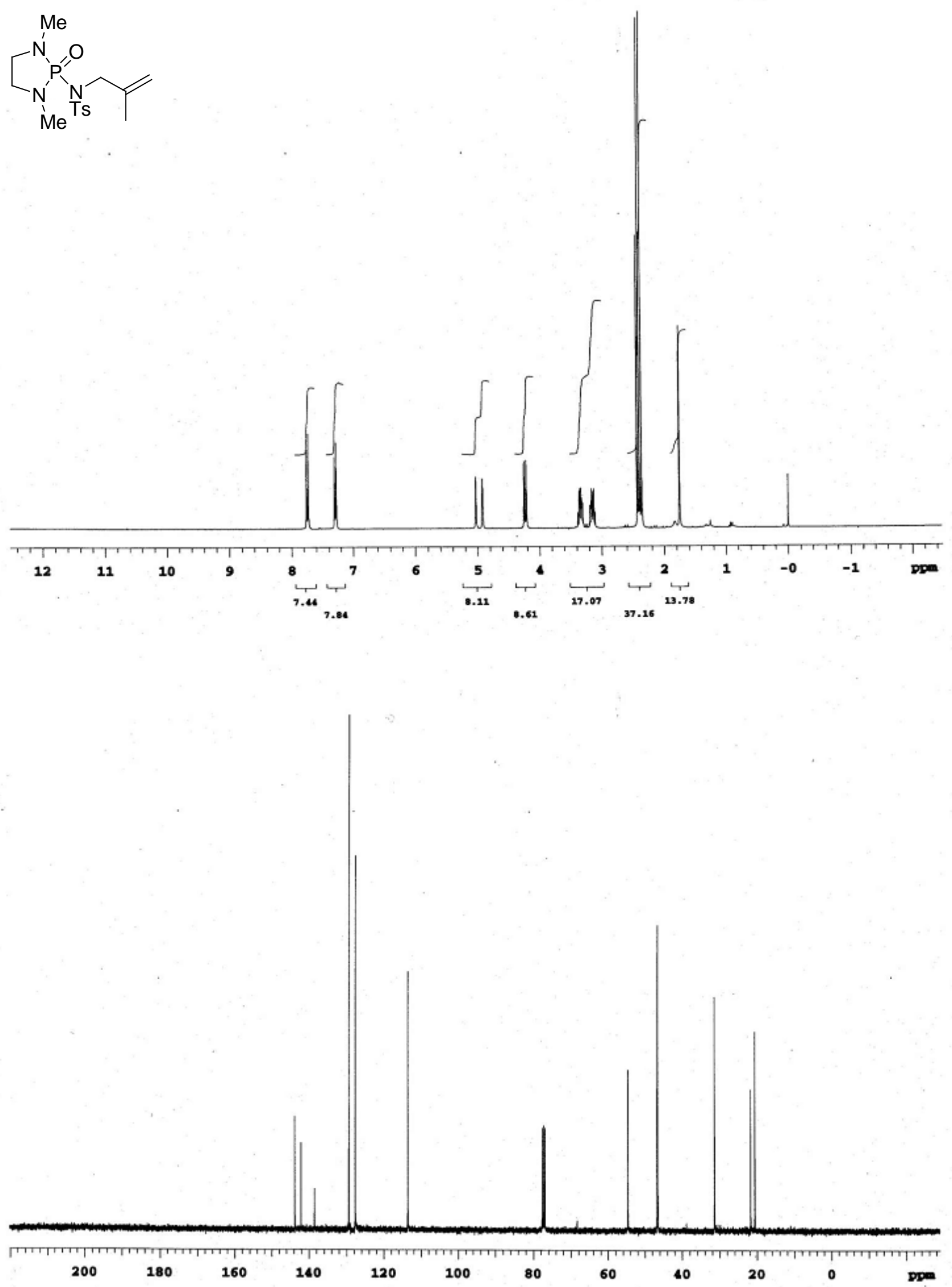

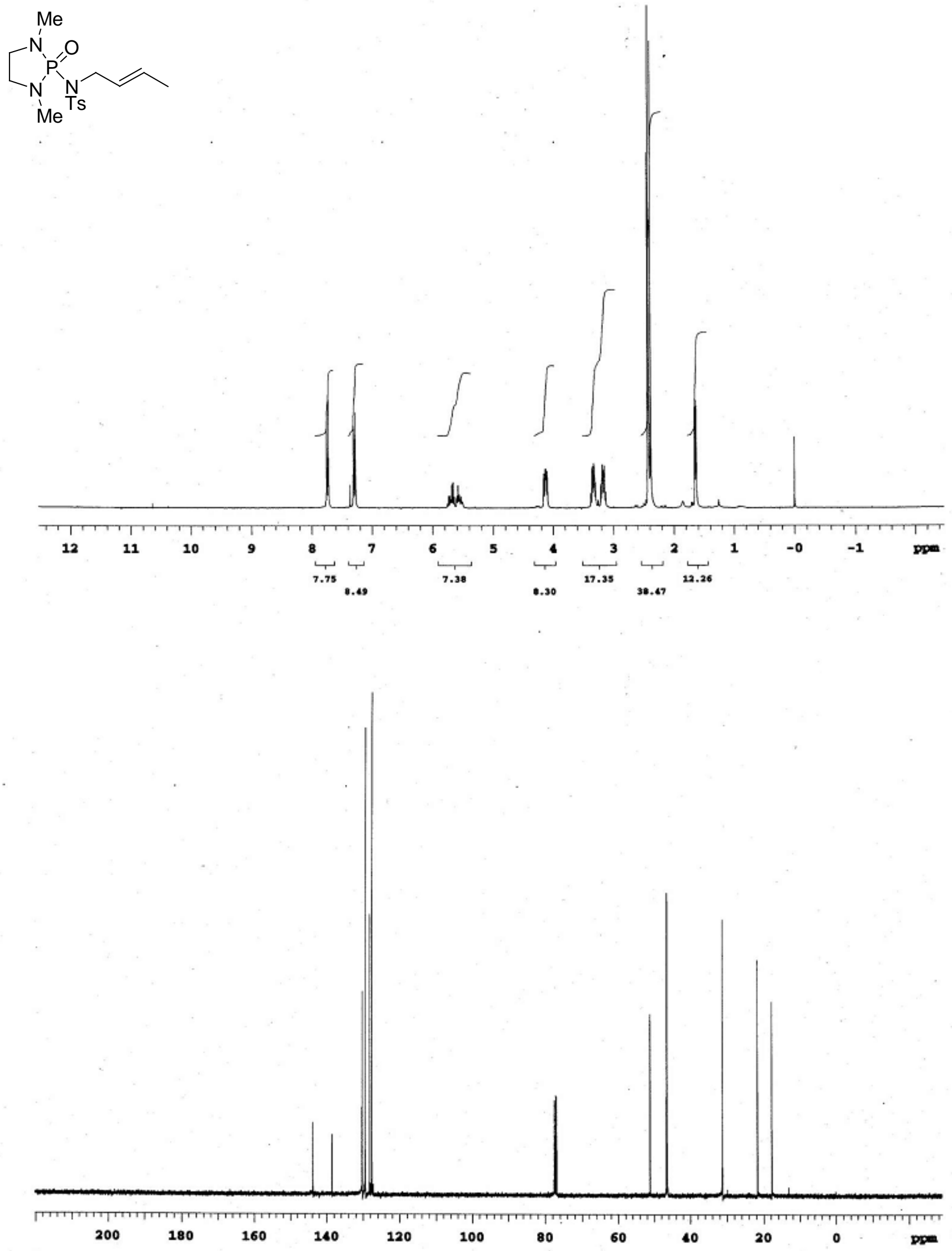
<smiles>CN1CCN(C)P1(=O)N(C1C=CCCC1)C1C=CCCC1</smiles>
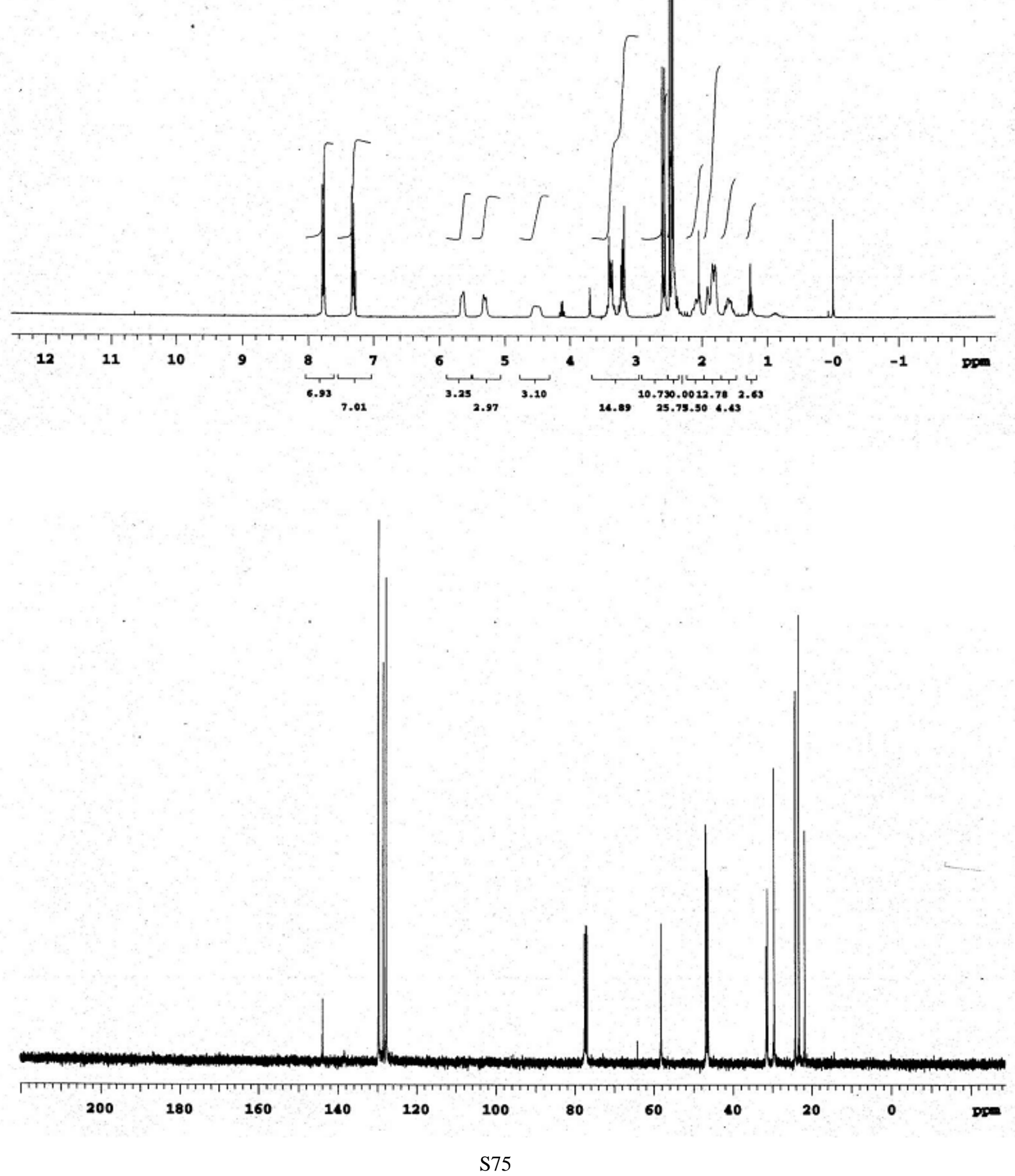
<smiles>CC/C=C/C(C)N(C)P1(=O)N(C)CCN1C</smiles>
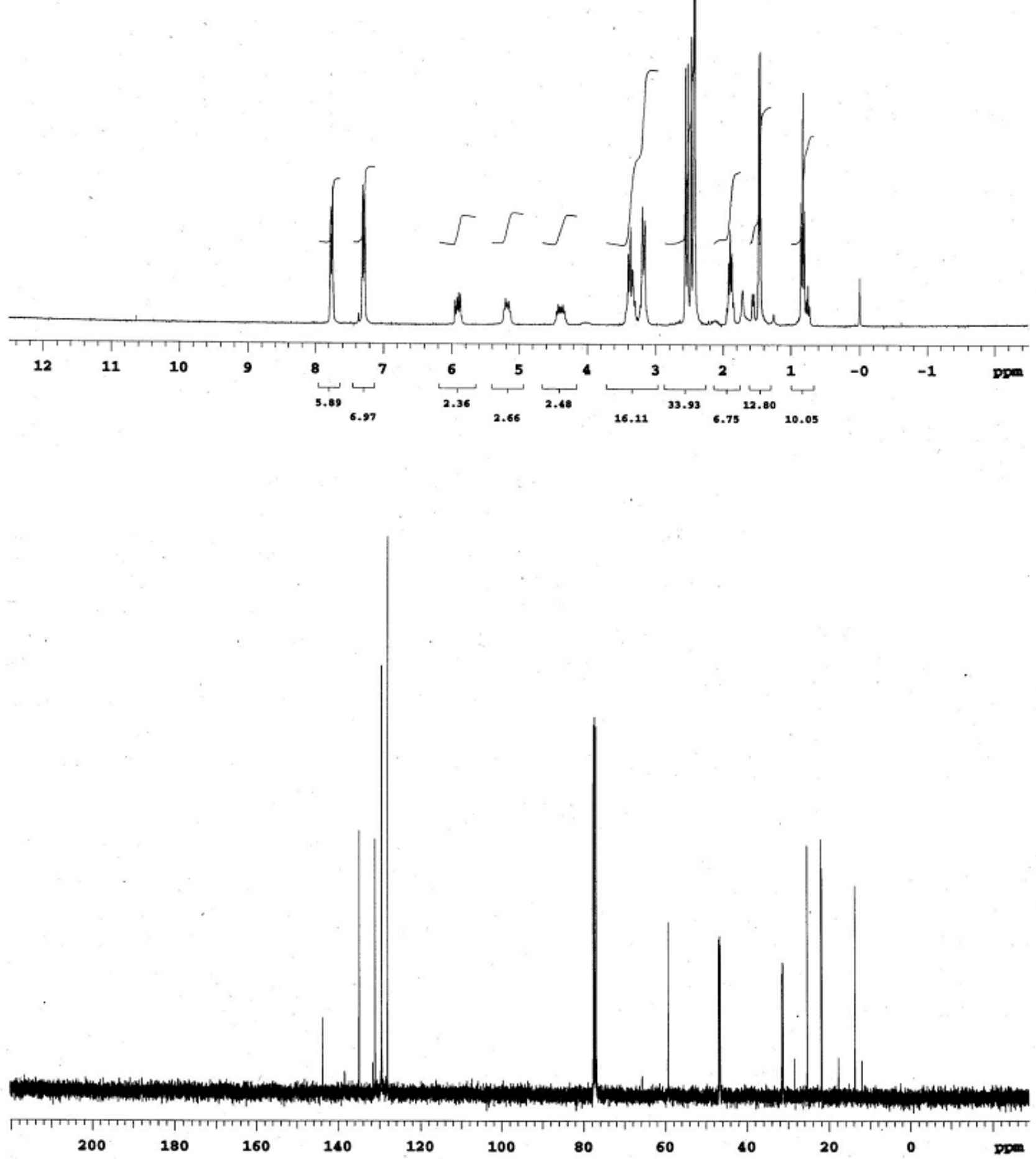

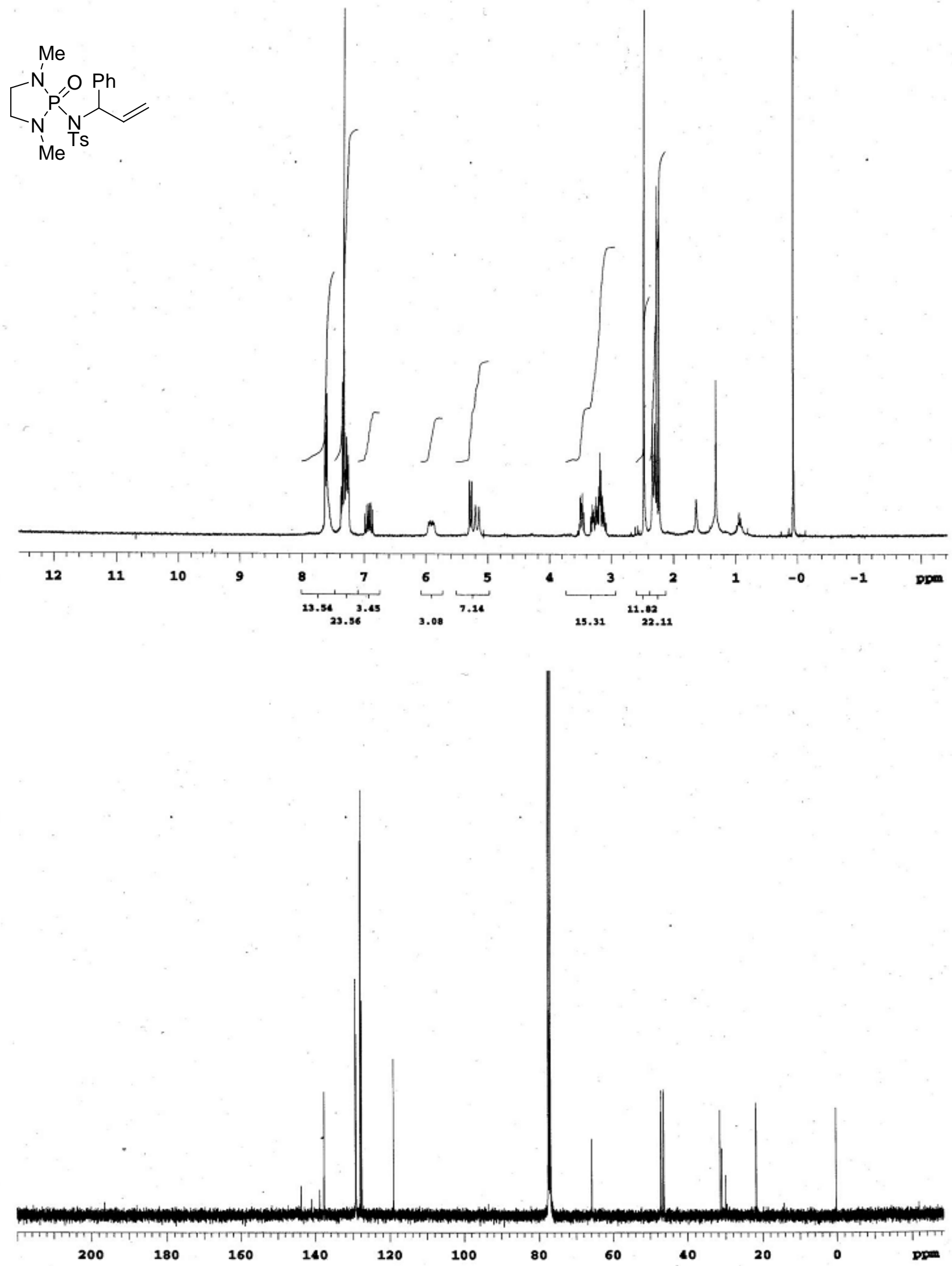

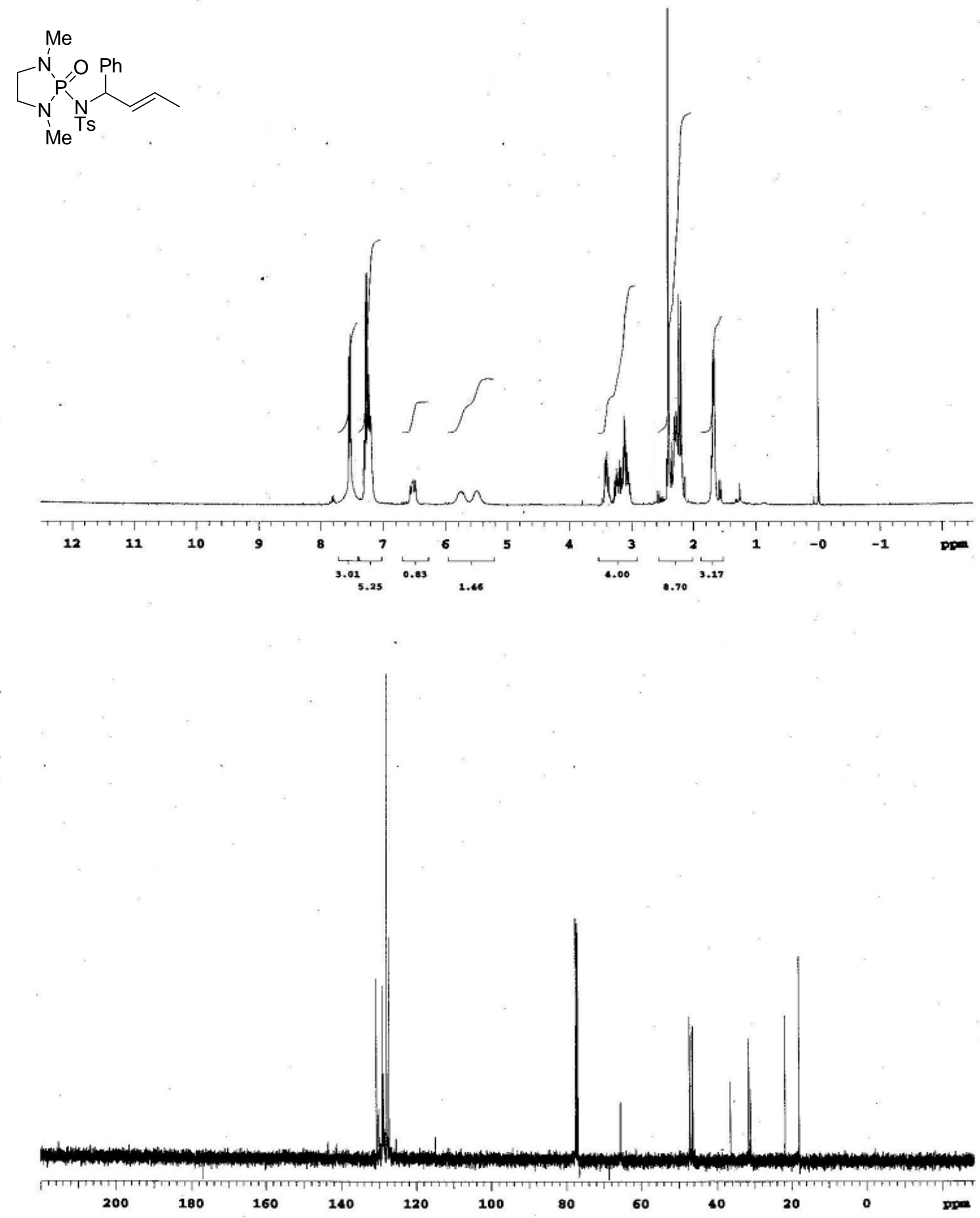

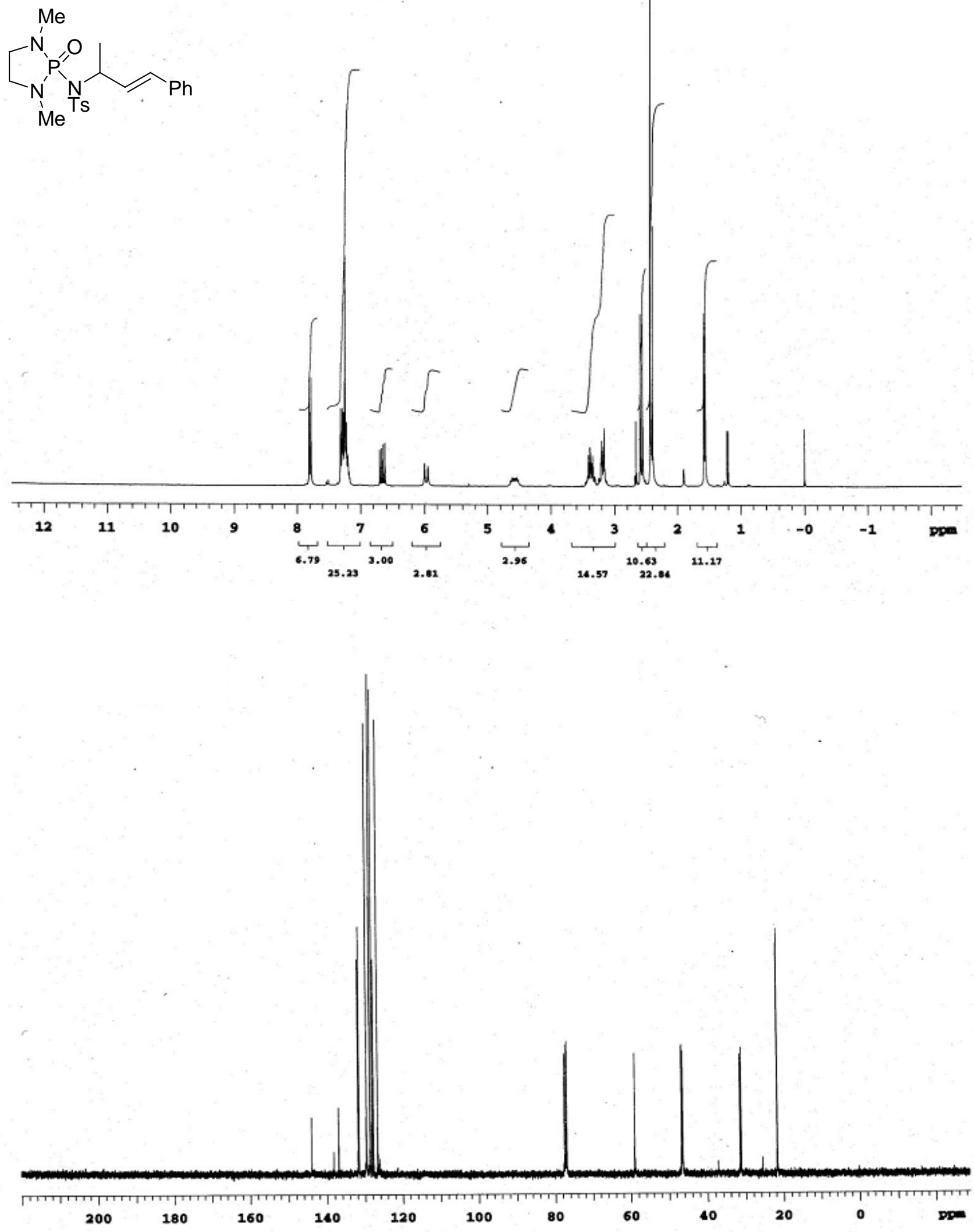

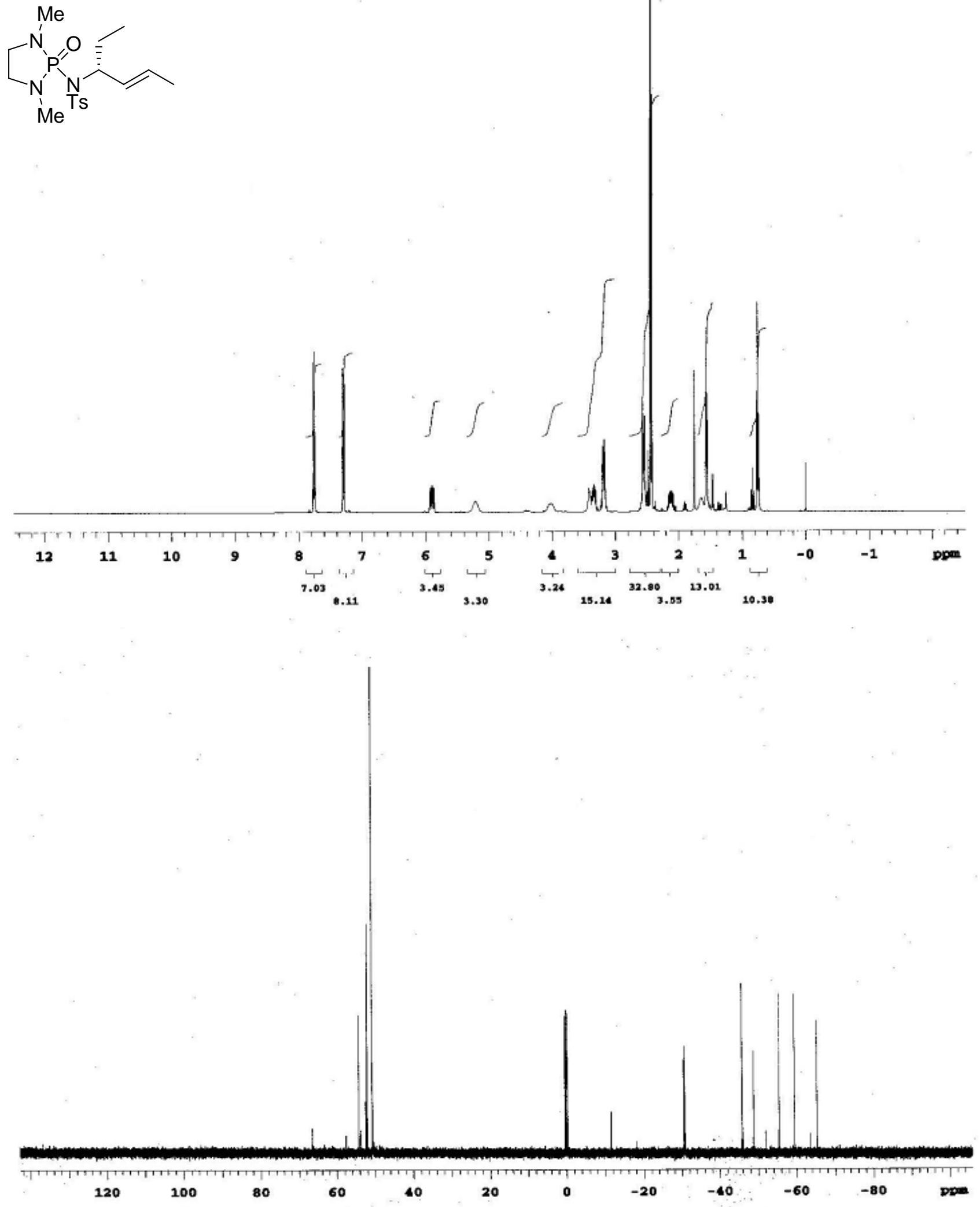

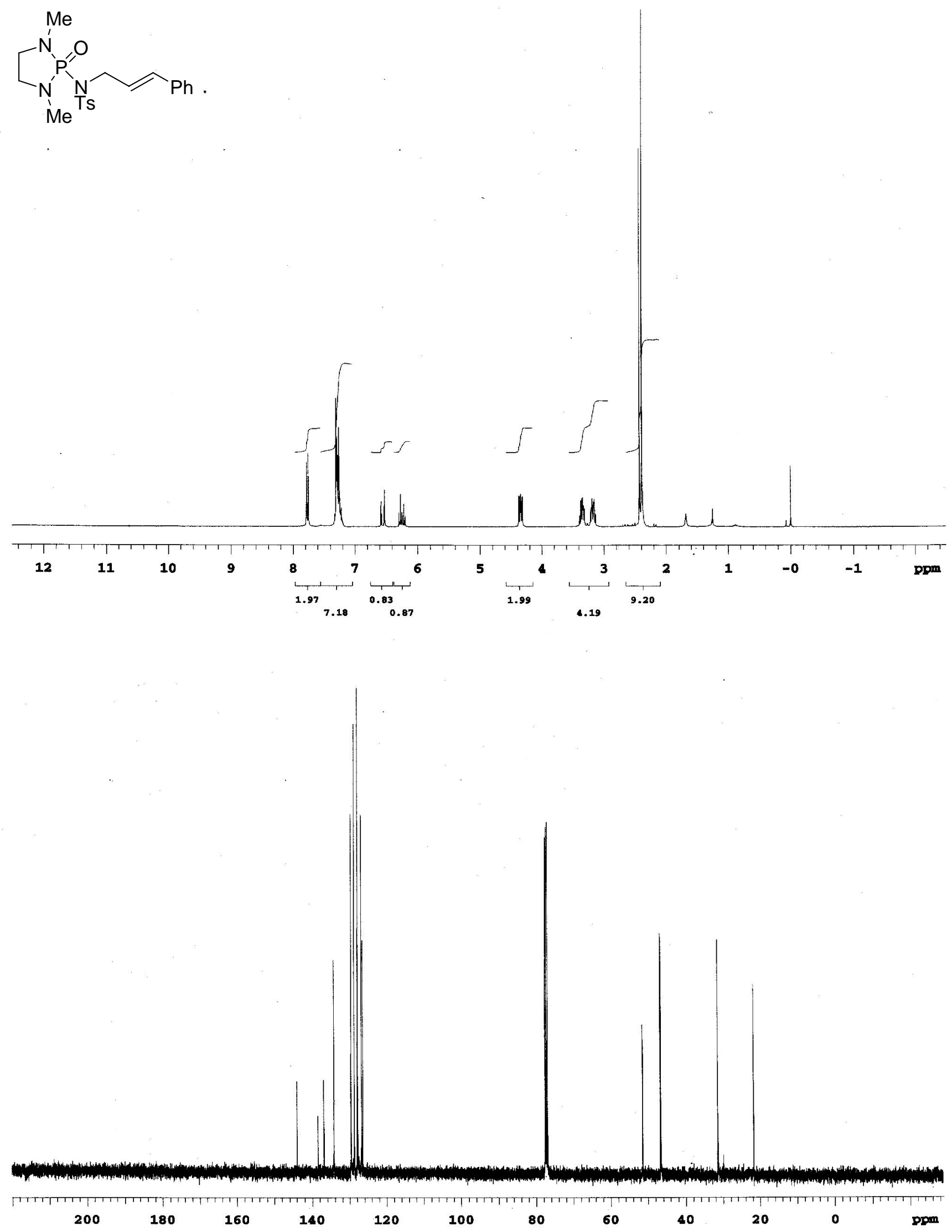

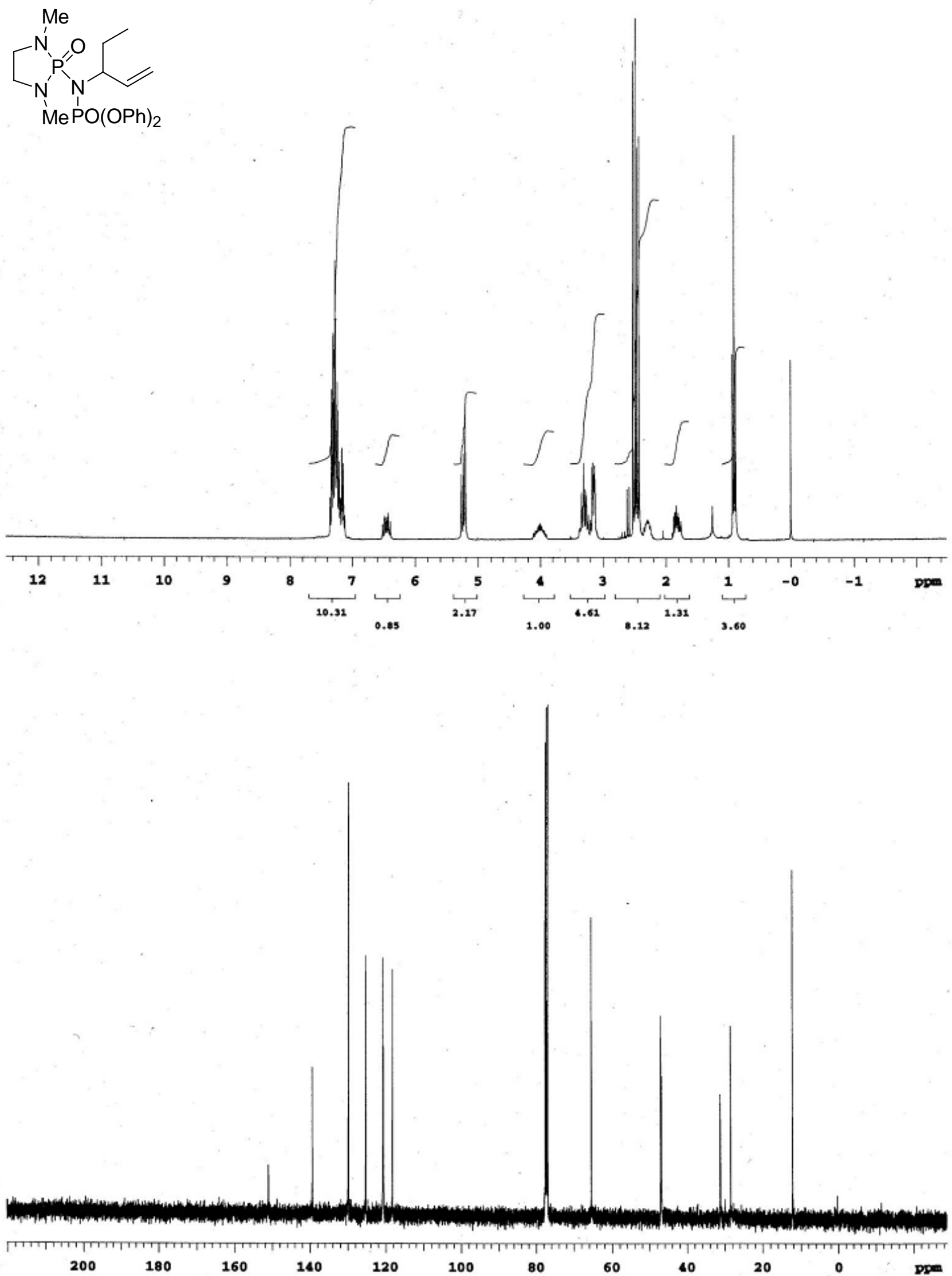

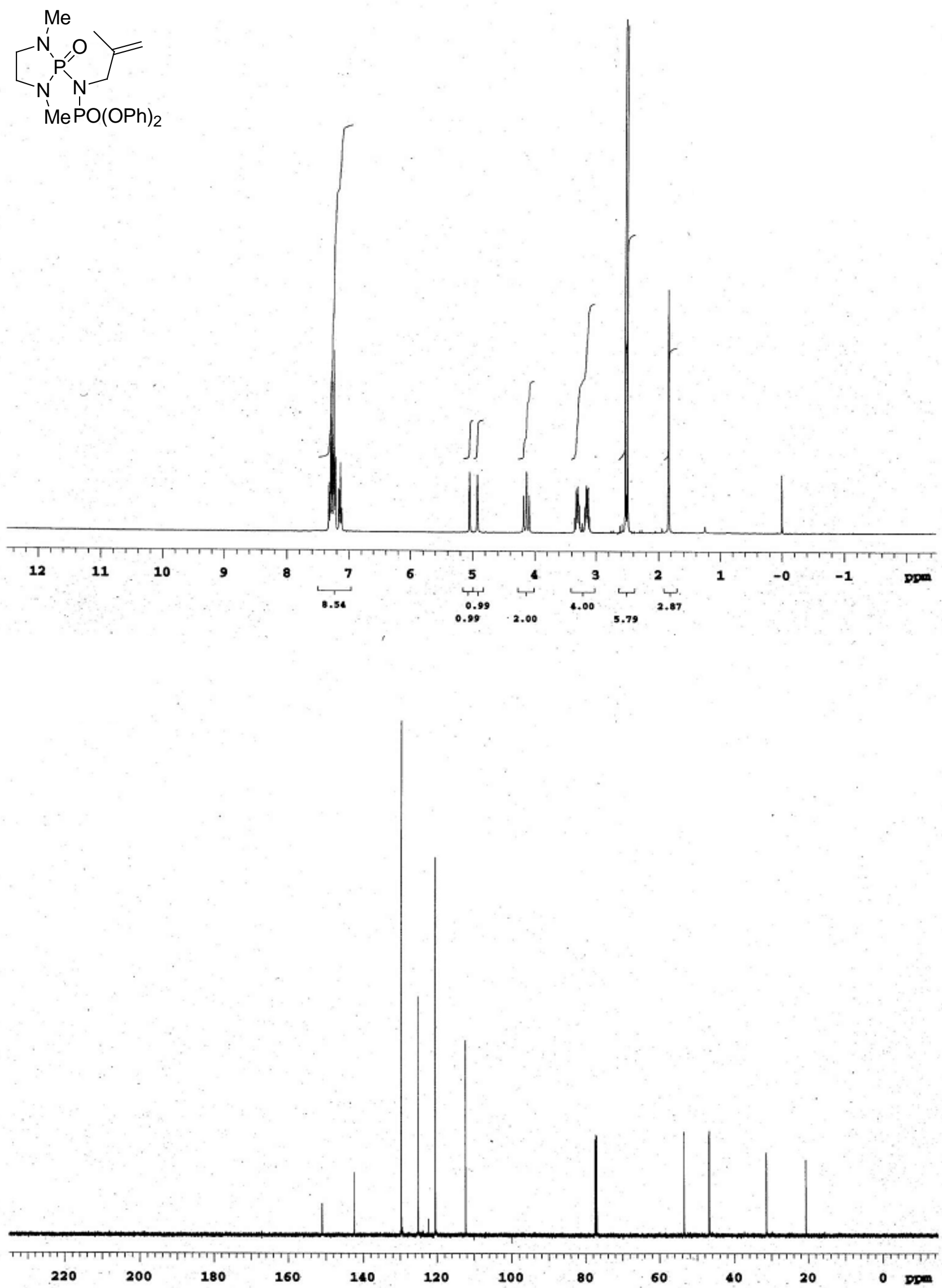

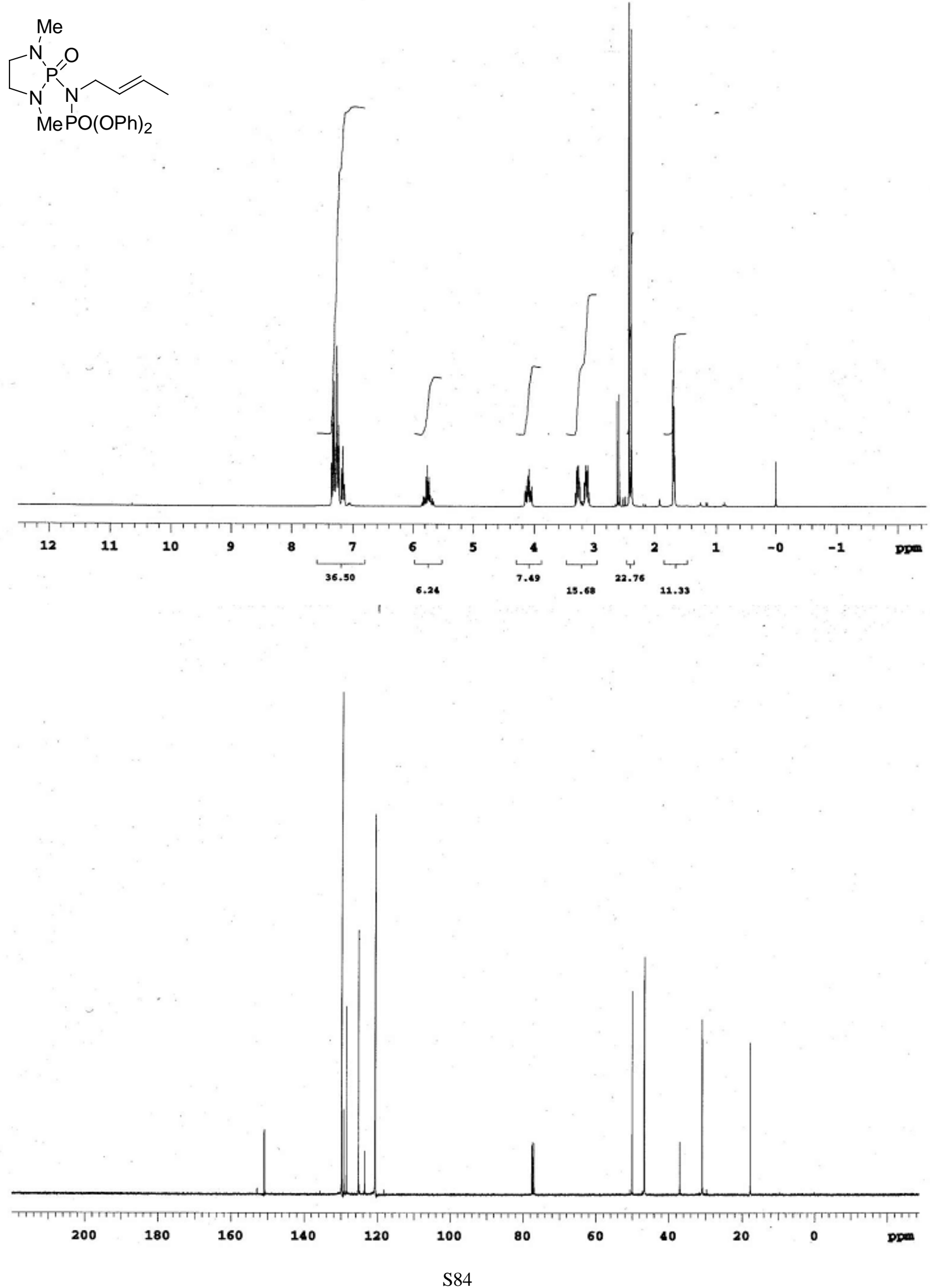

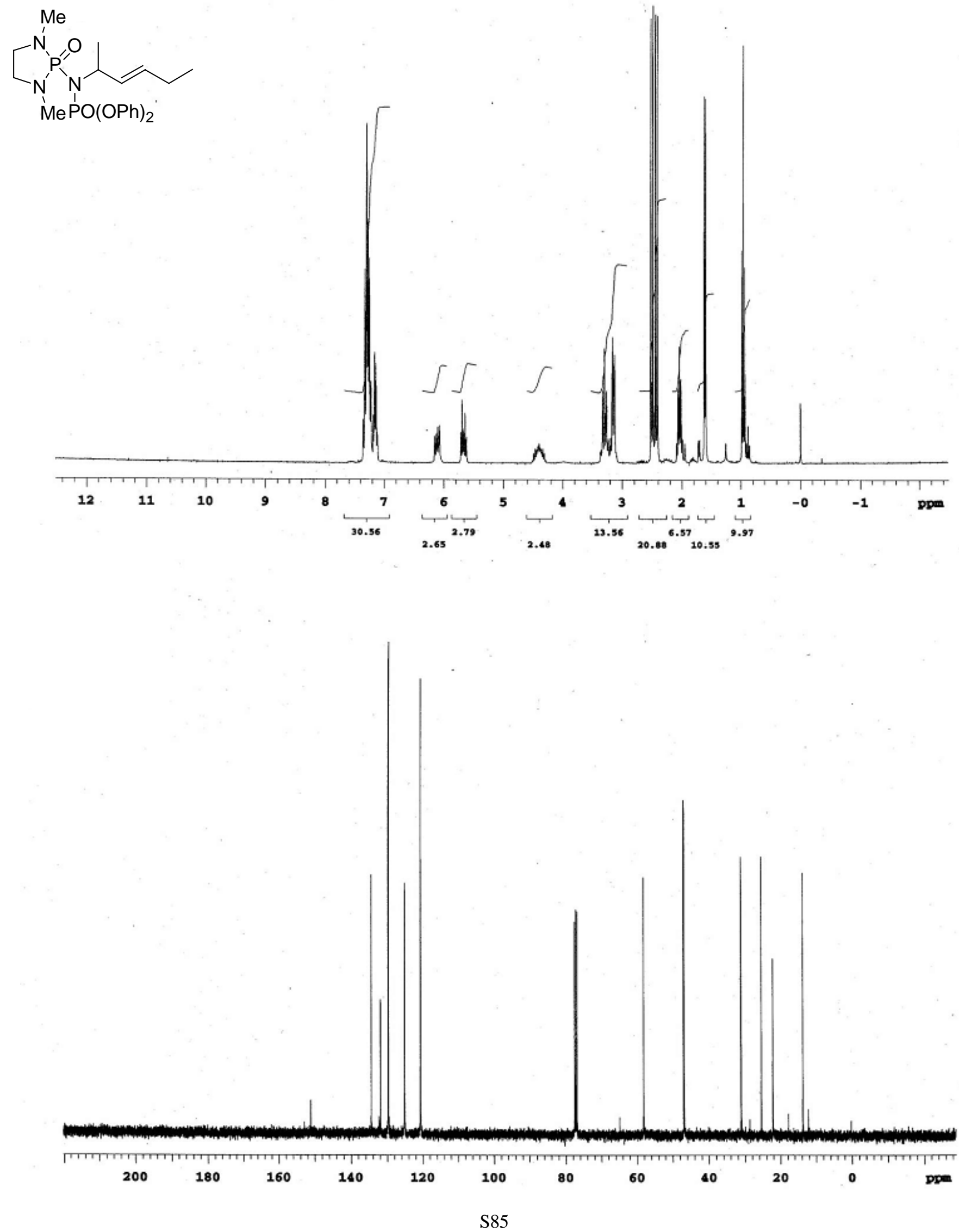

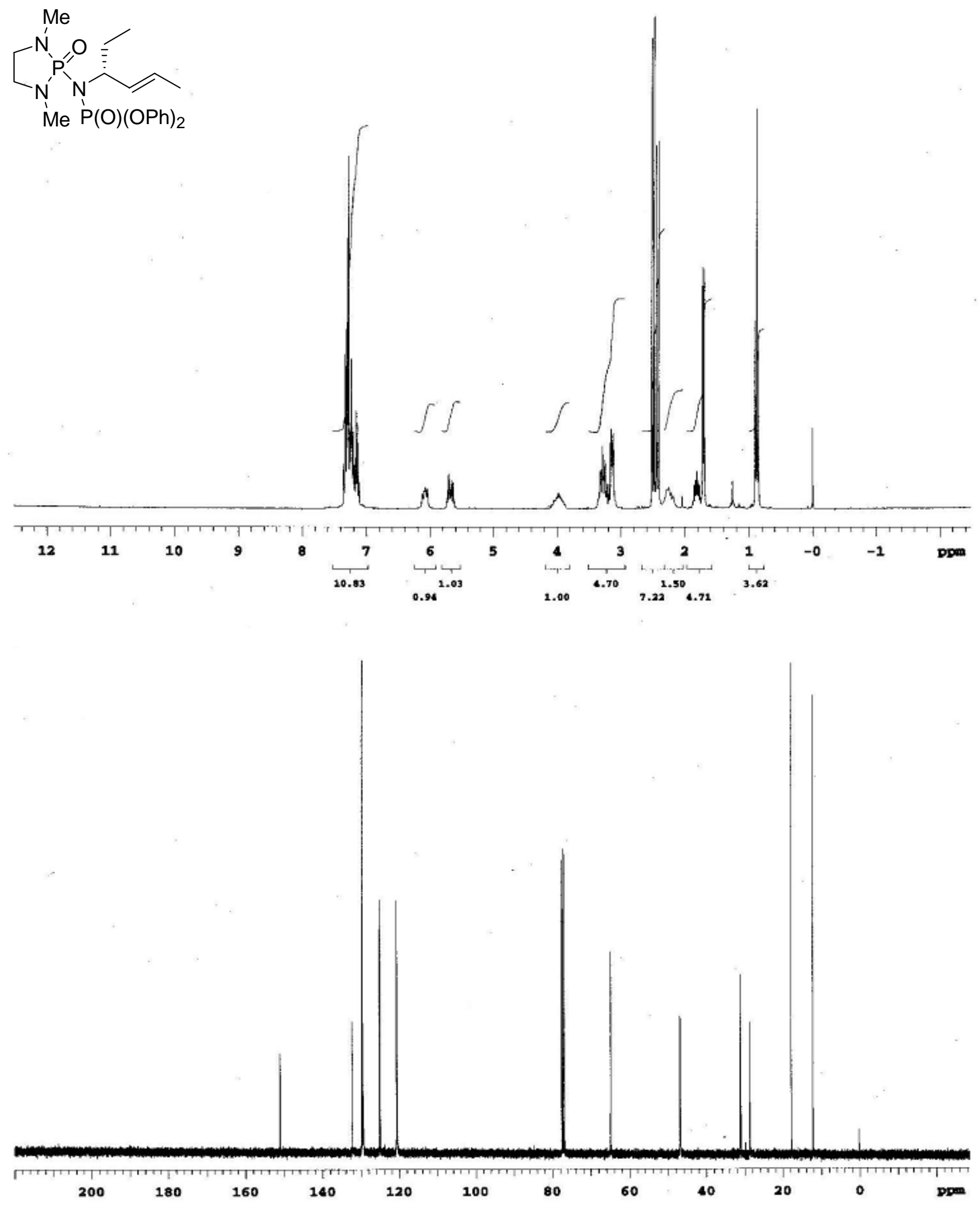

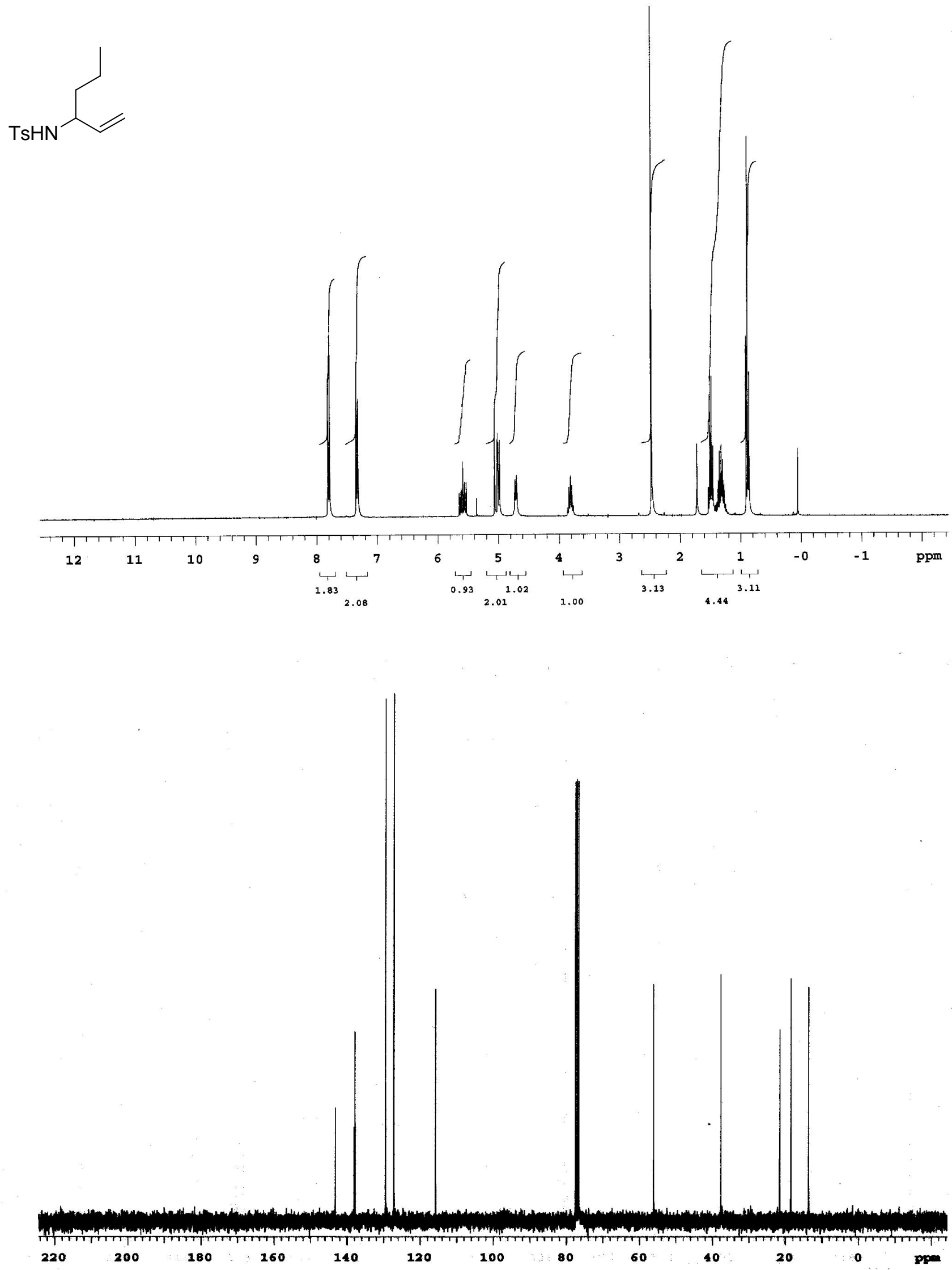
Chiralcel OD, 1PrOH:Hex;5:95, 1mL/min

Deprotected Tosylamine from Cis substrate

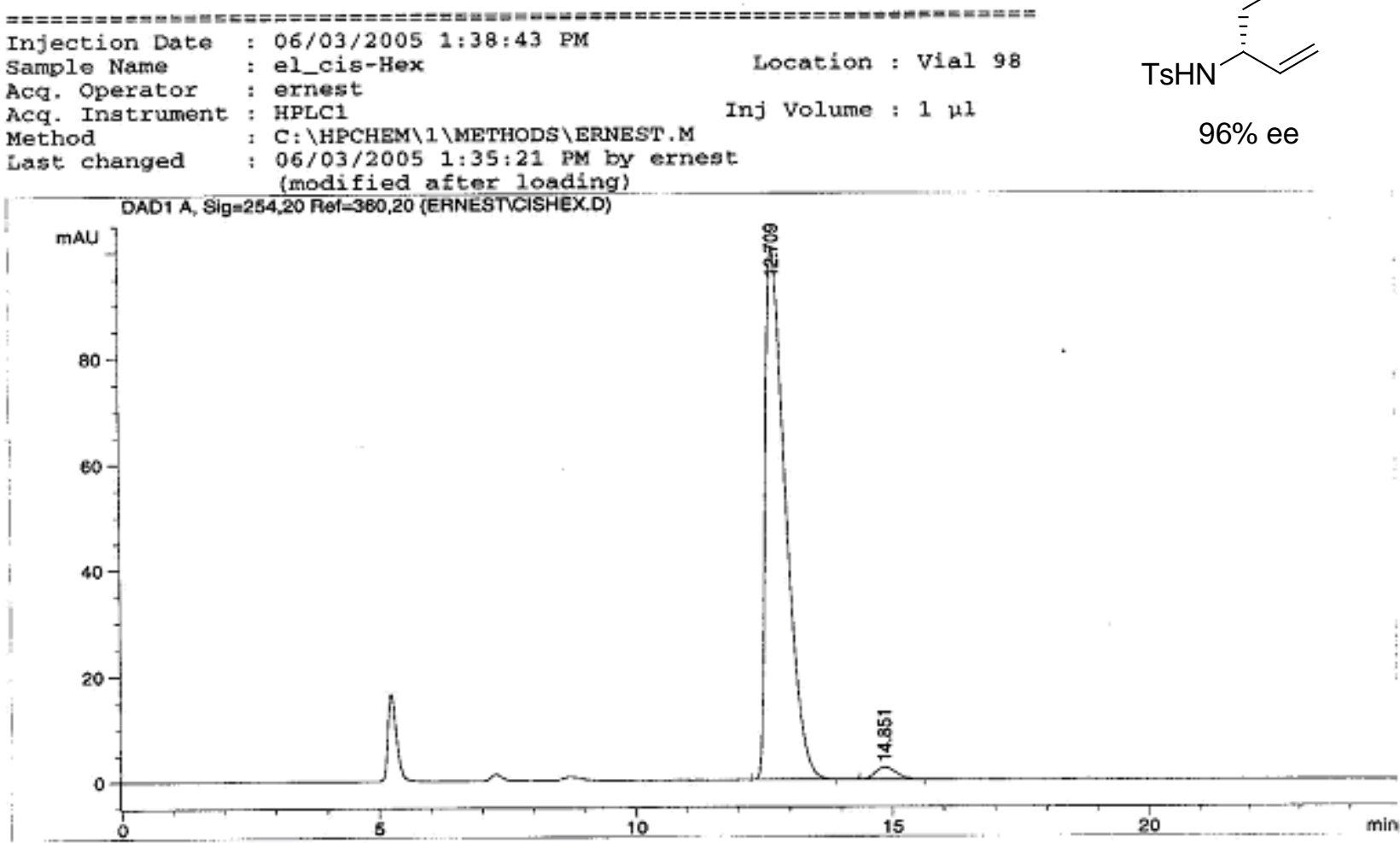

OD with guard, 95:5 hexanes: IPA, $30 \mathrm{degc,} 1 \mathrm{~mL} / \mathrm{min}$ Deprotected Hex from Trans

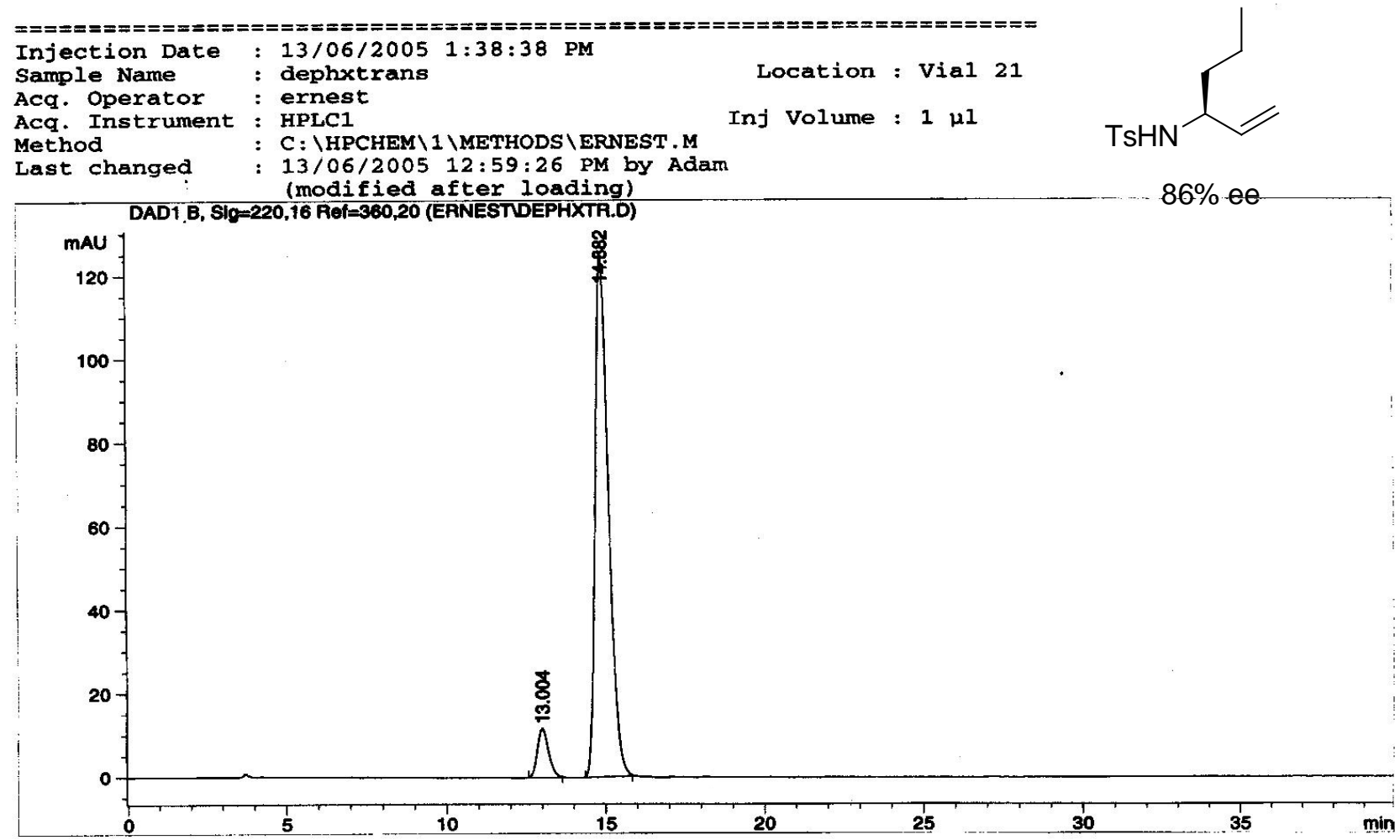



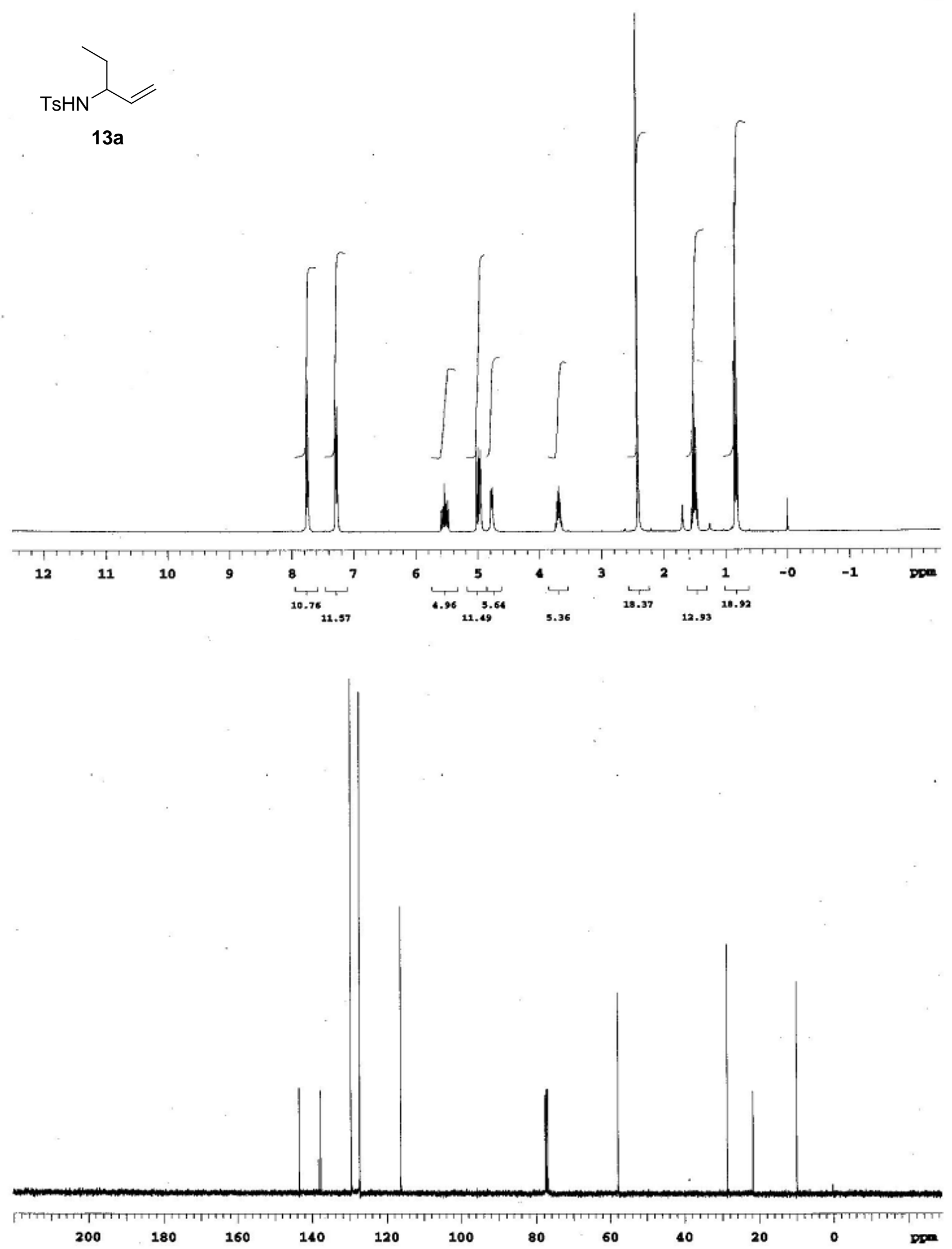
Chiralcel AD with guard, 96:04 Hexanes/LProh, 30C, $1 \mathrm{~mL} / \mathrm{m}$

in

Deprotected Et/Ts from cia

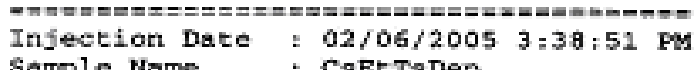

Sample Name : CaBtTaDep

Acq. Operator : exnest

Acc. Instrument - HPTC2

Nethod : C: \HPCHEM 1 \HETHODS \ERWEST $\boldsymbol{x}$

Last changed $29 / 05 / 20053: 09: 49$ FM by Ernest

Location : Vial 1

TsHN

Inj Volume : $1 \mu 1$

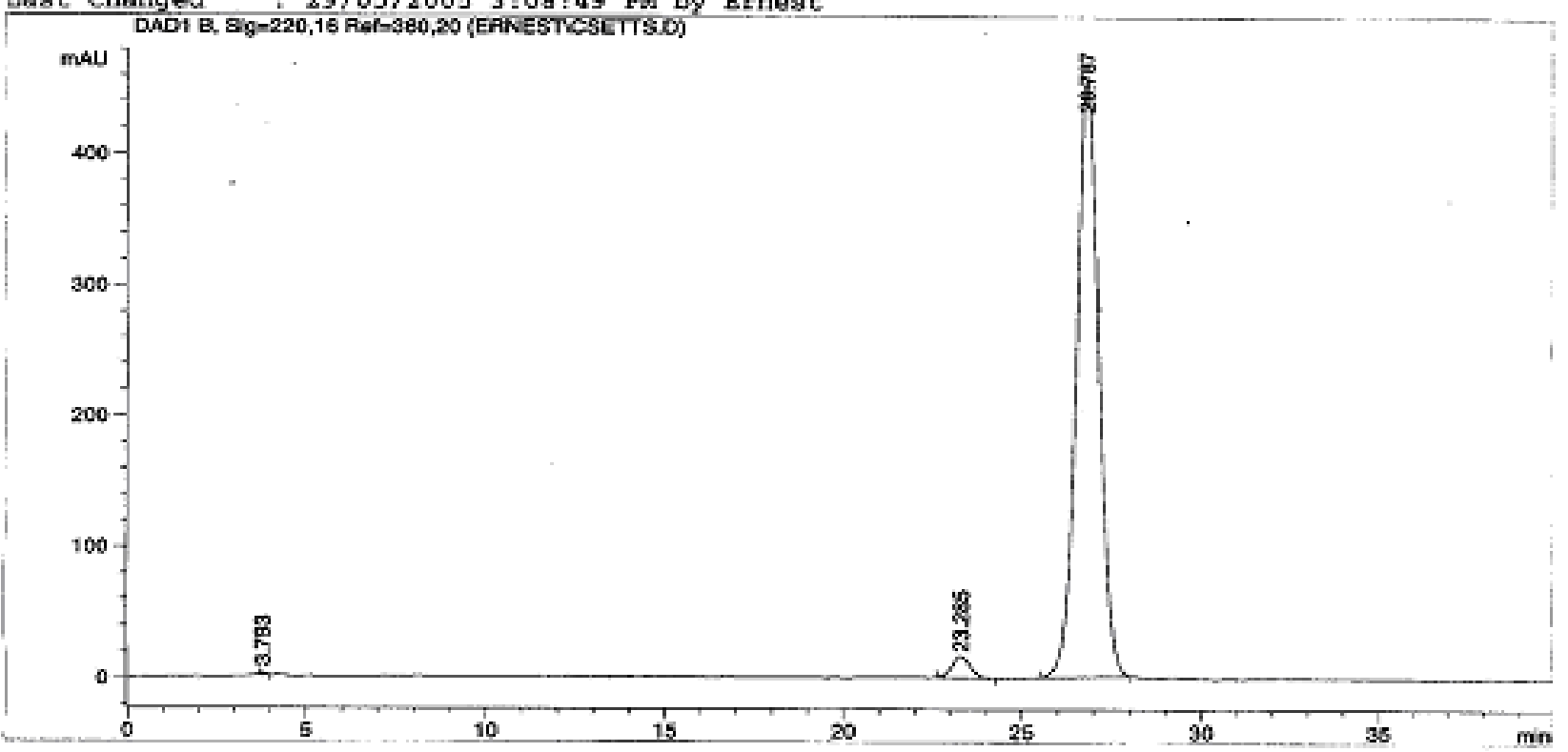

Chiralcel AD with guard, 96:04 Hexanes/iProH, 30C,1mL/m

in

Deprotected Et from Trans

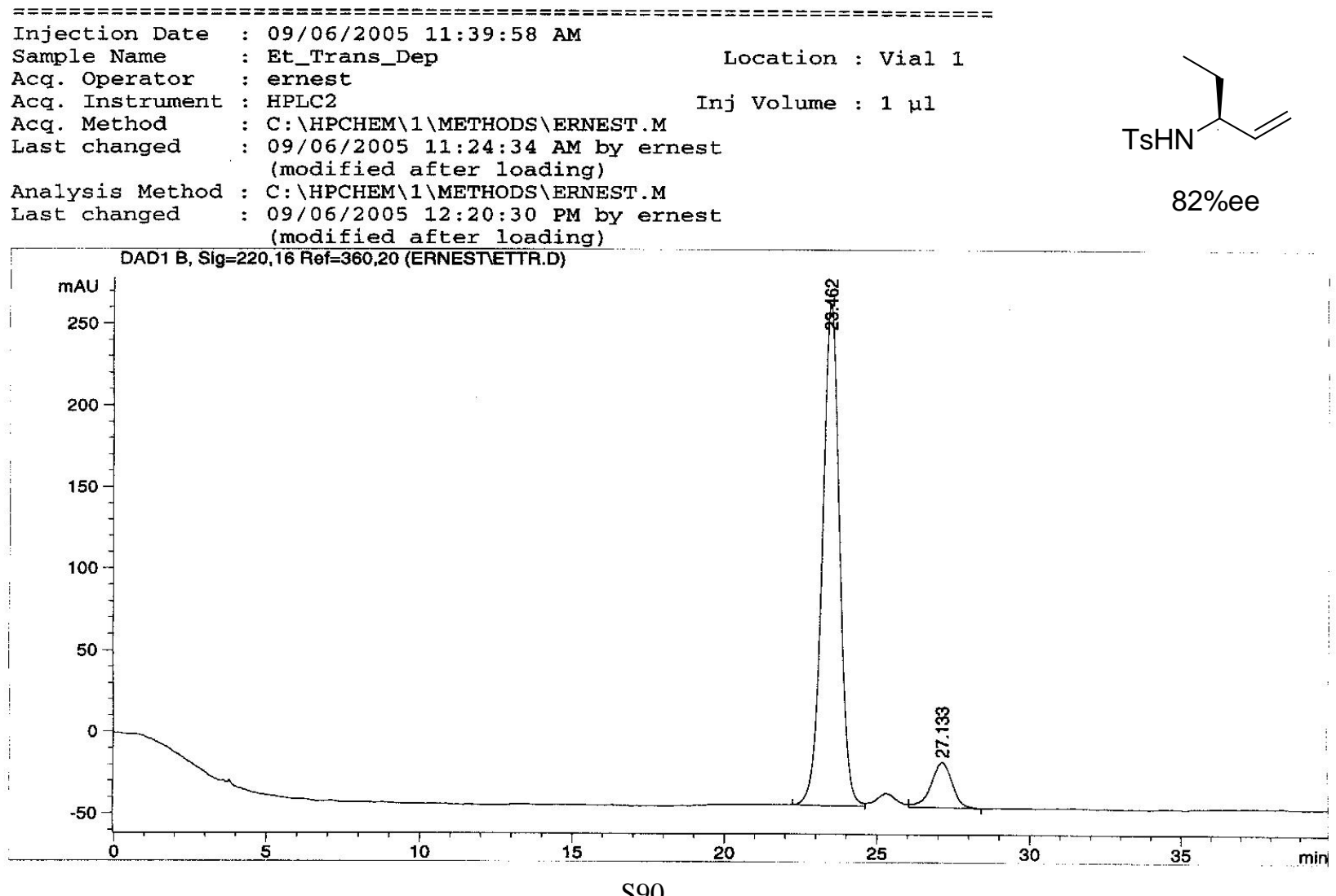



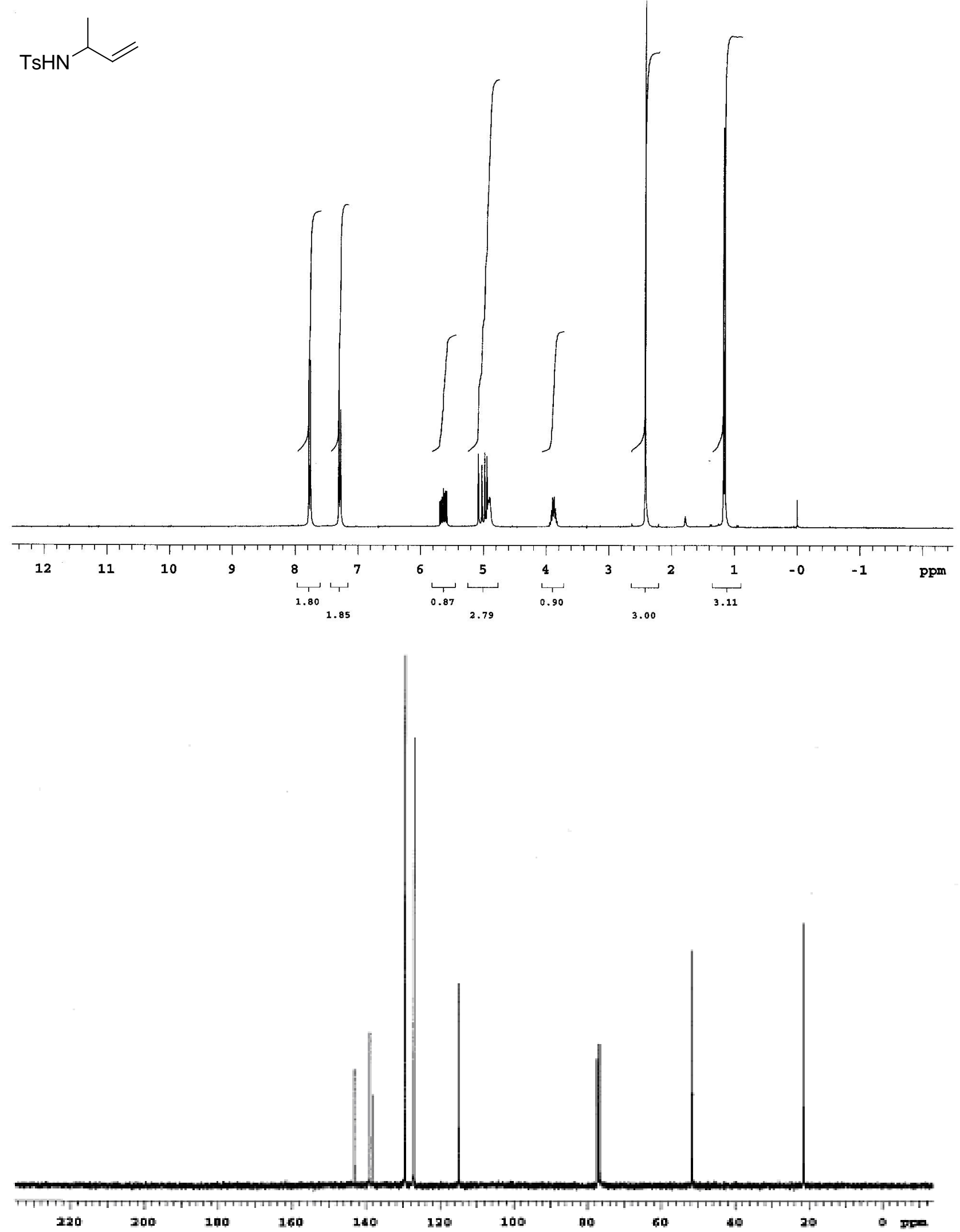


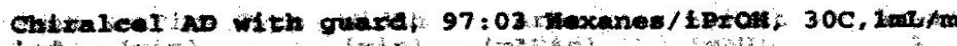

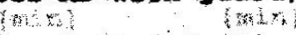
$\left(x^{2}+2<\leq ;\right.$ ant

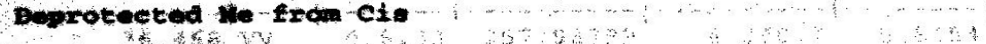

Injection Date: : $08 / 06 / 20055: 36: 07 \mathrm{RH}$

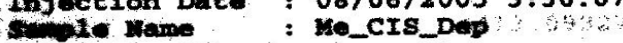

Aca, operator : erneat

Location : Vial 1

Herce 2 hut

Inj Volume : 1 Hl

TsHN $\stackrel{\vdots}{\vdots}$

$91 \%$ ee

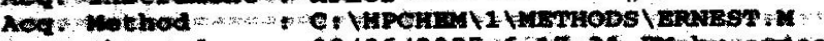

Last changed : $08 / 06 / 2005$ / $17: 31$ pubs ented (modified after londing)

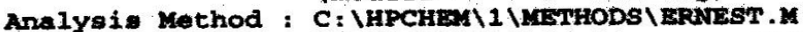

wast changed : 08/06/2005 6:34:58 PA by ernest

(modified after loading)

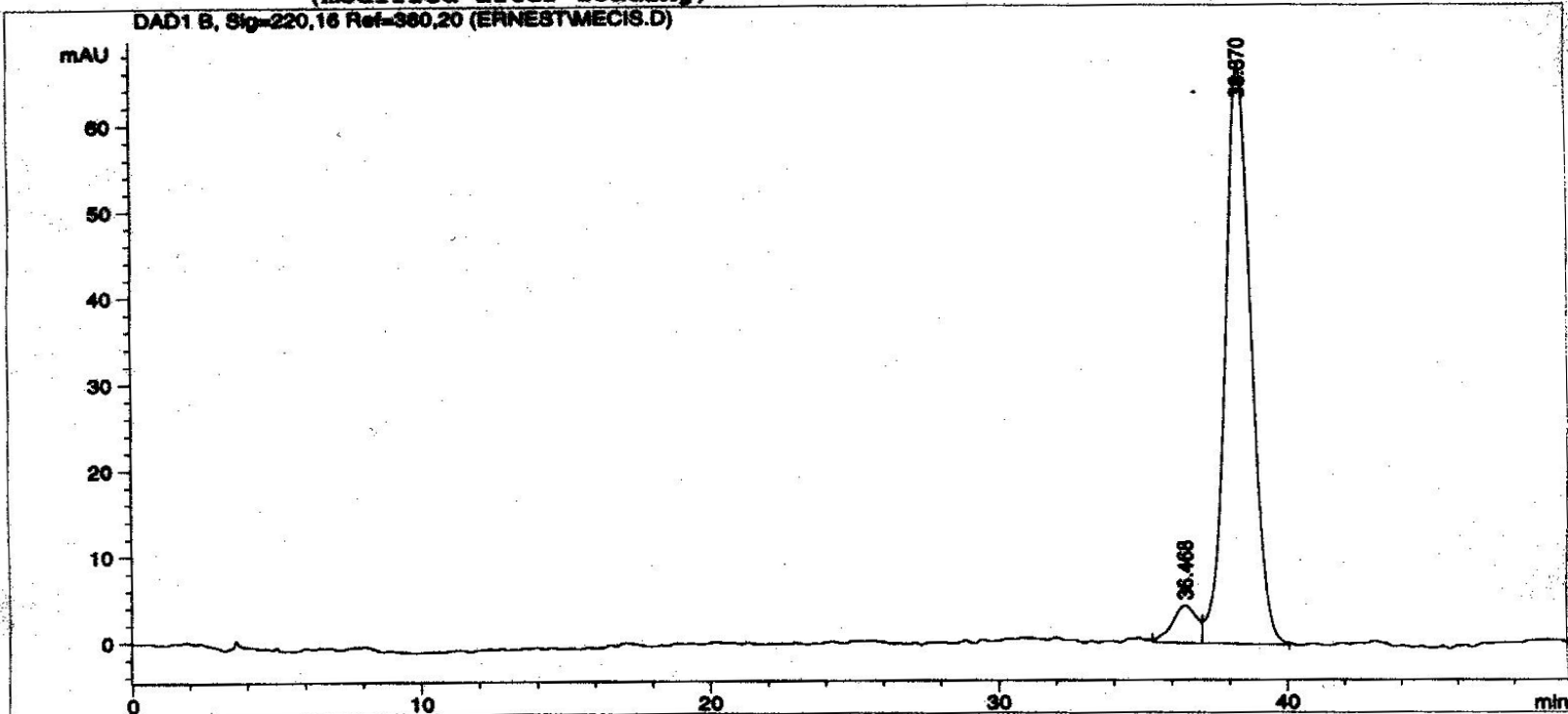

Chiralcel AD with guard, 97:03 Hexanes/iProH, 30c,1mL/m in

Deprotected Me from Trans
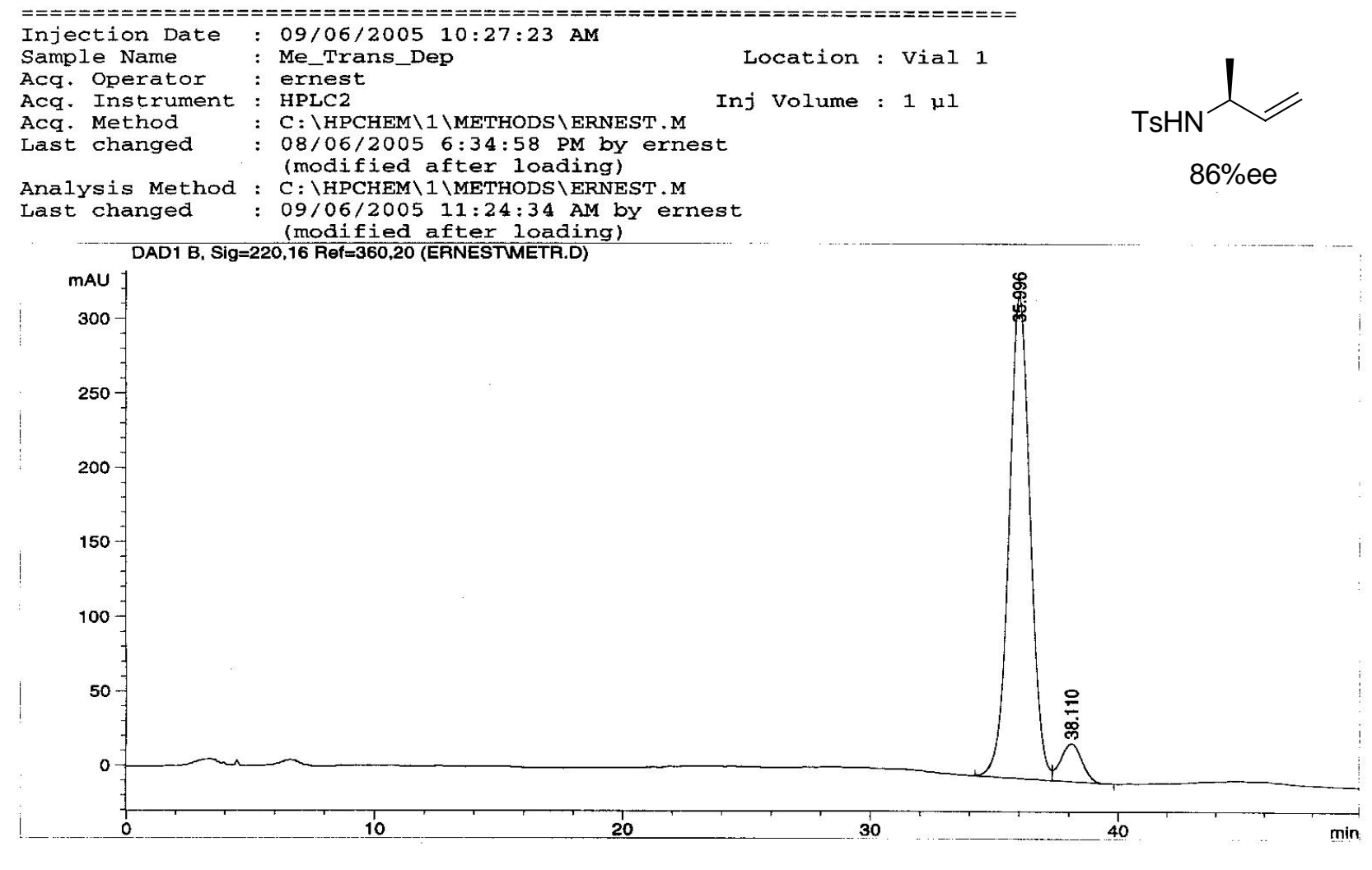

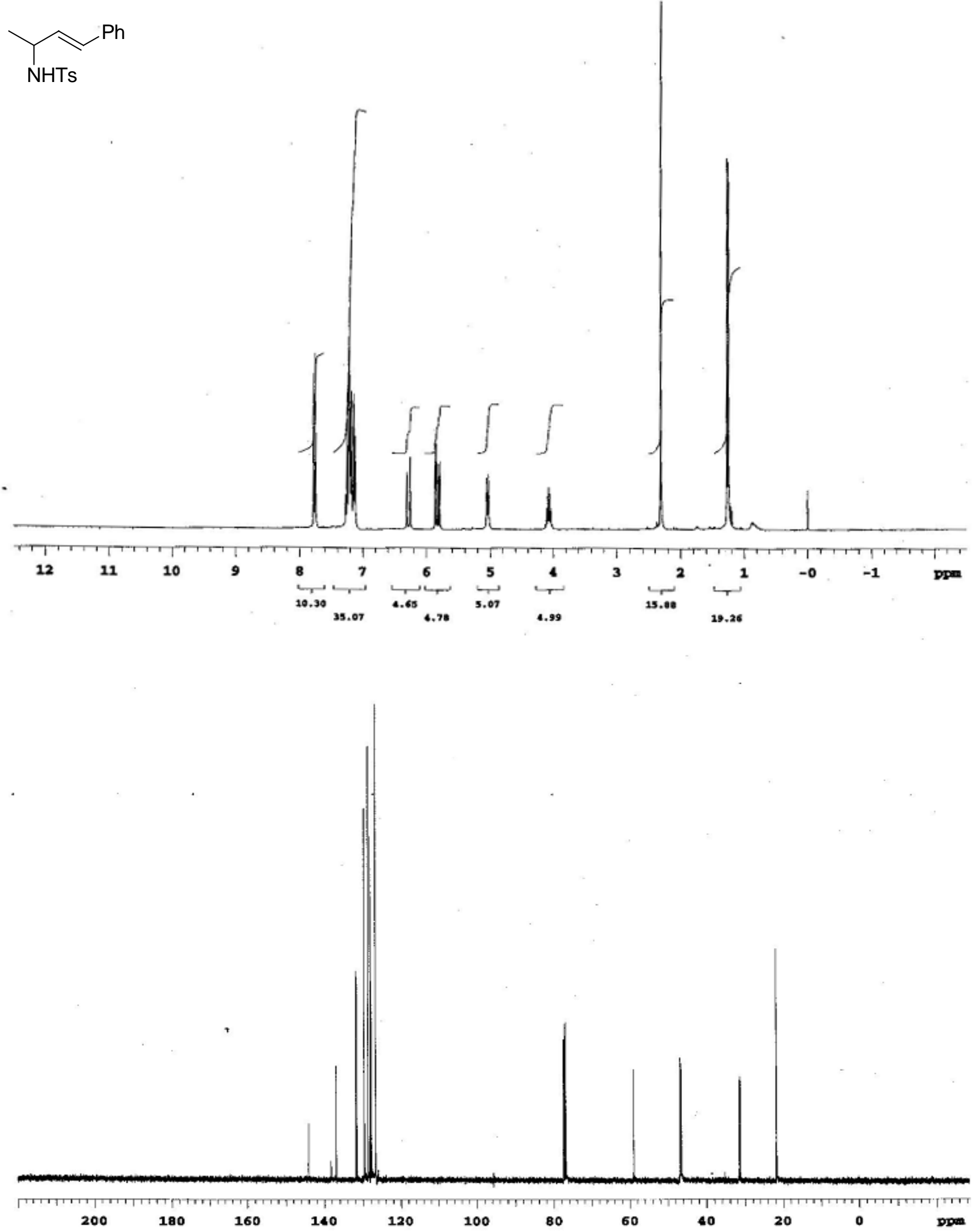

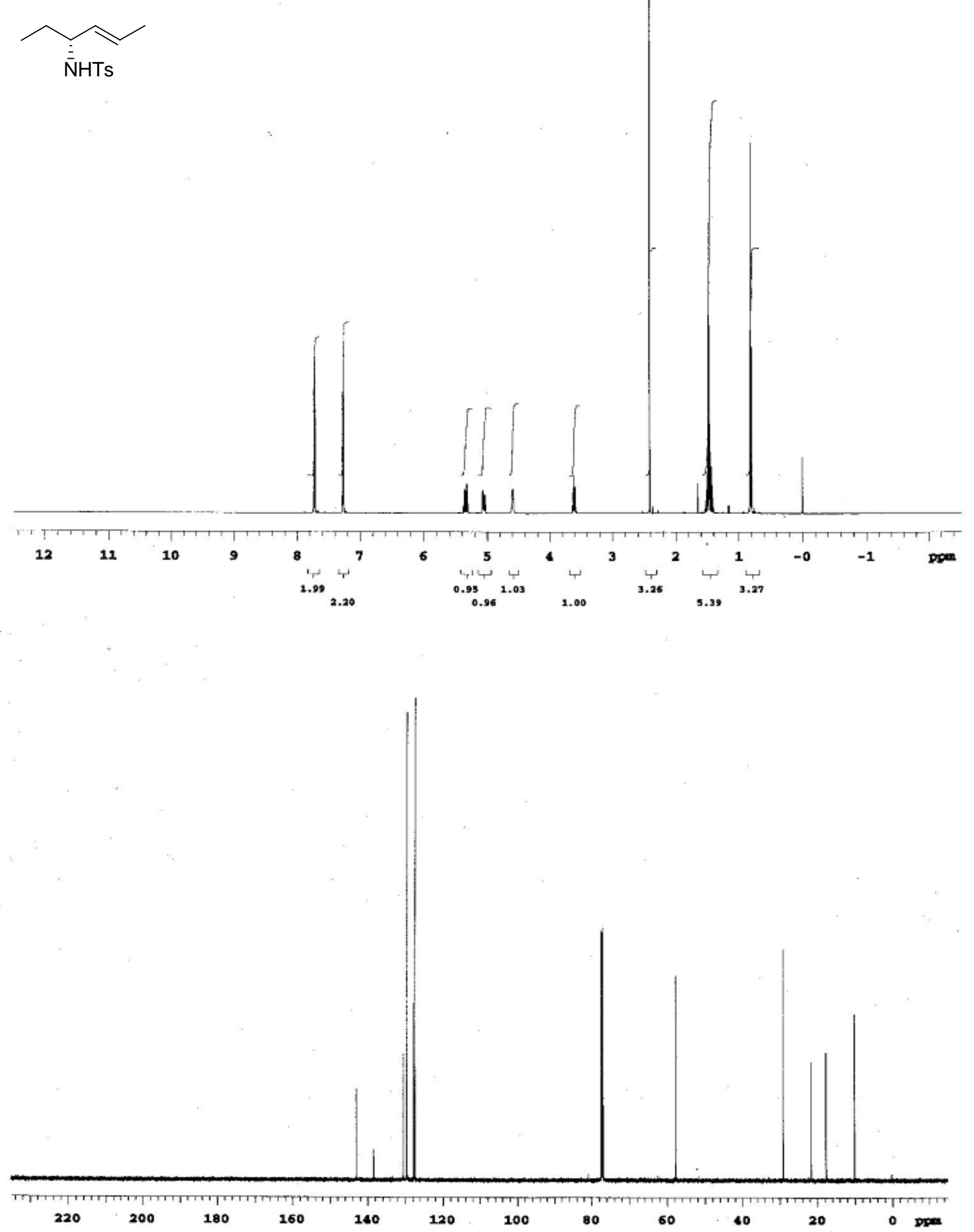

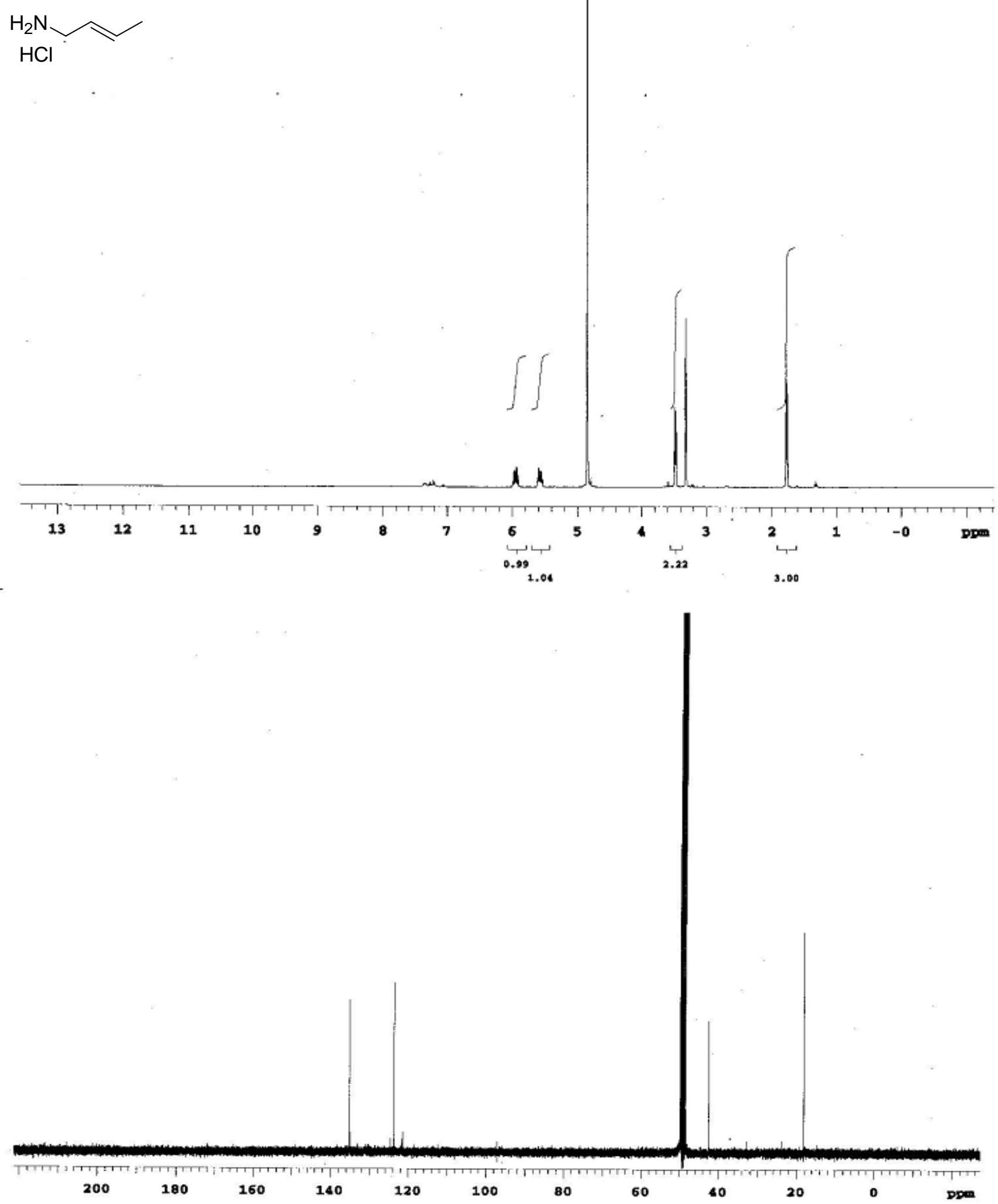

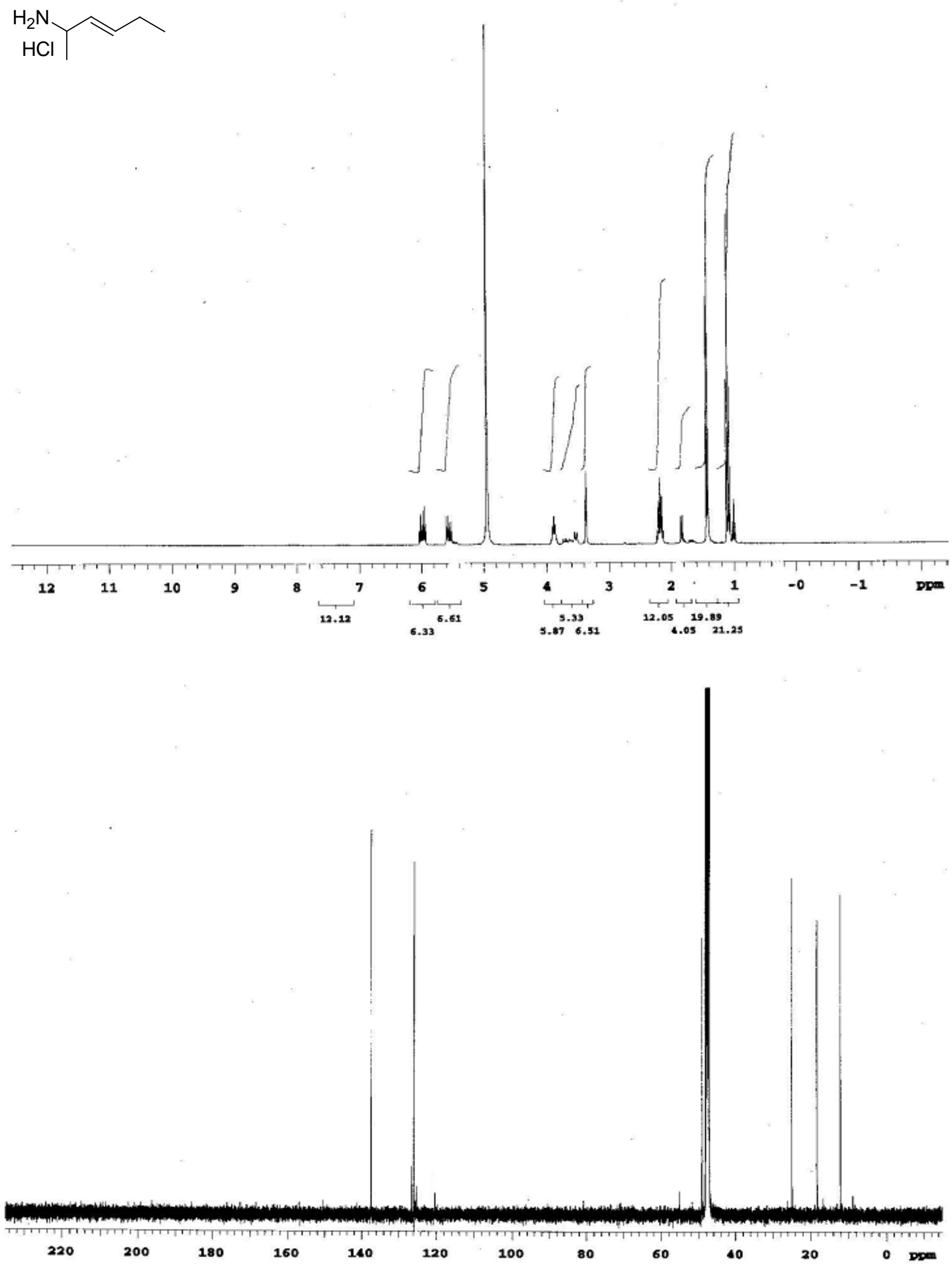

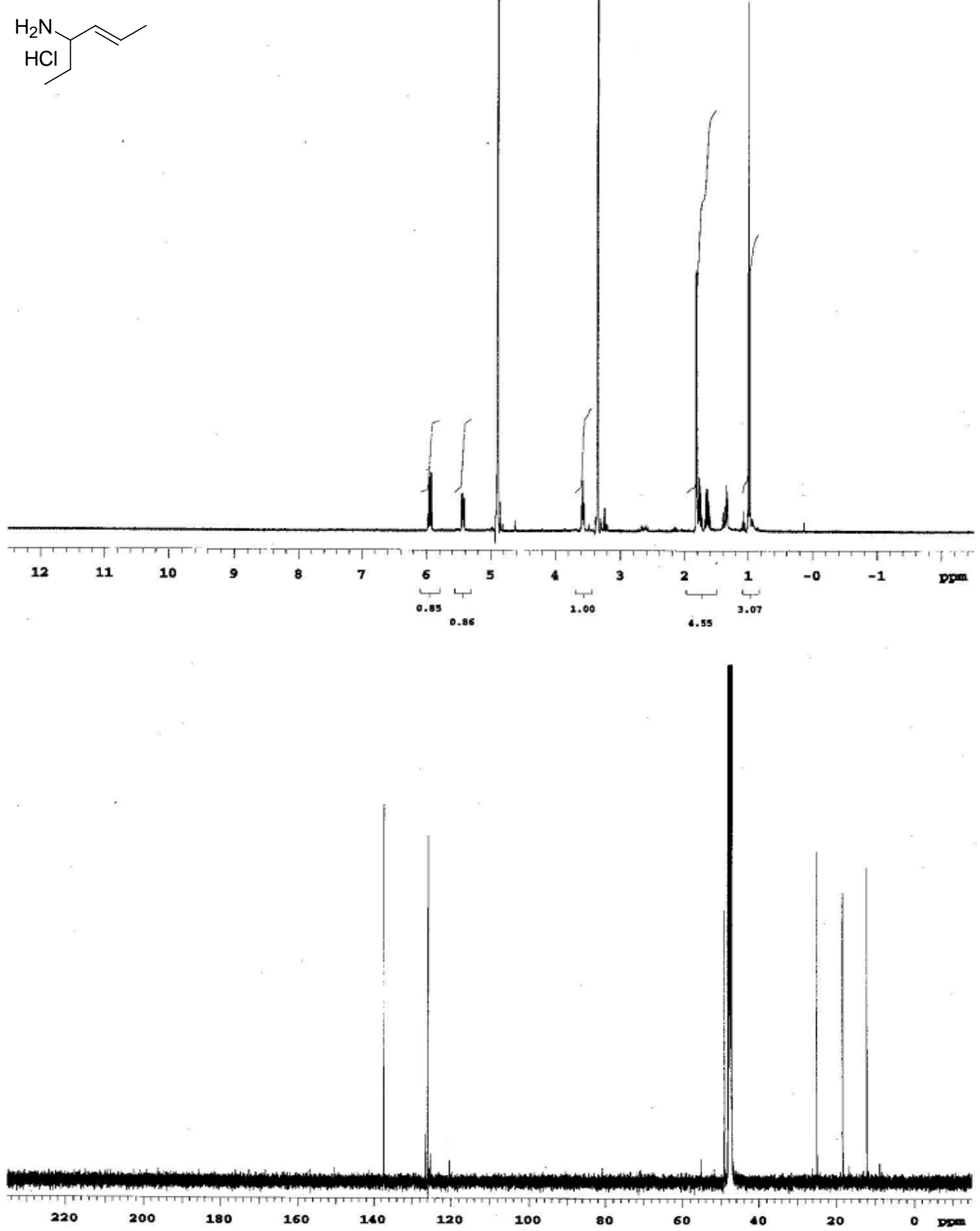

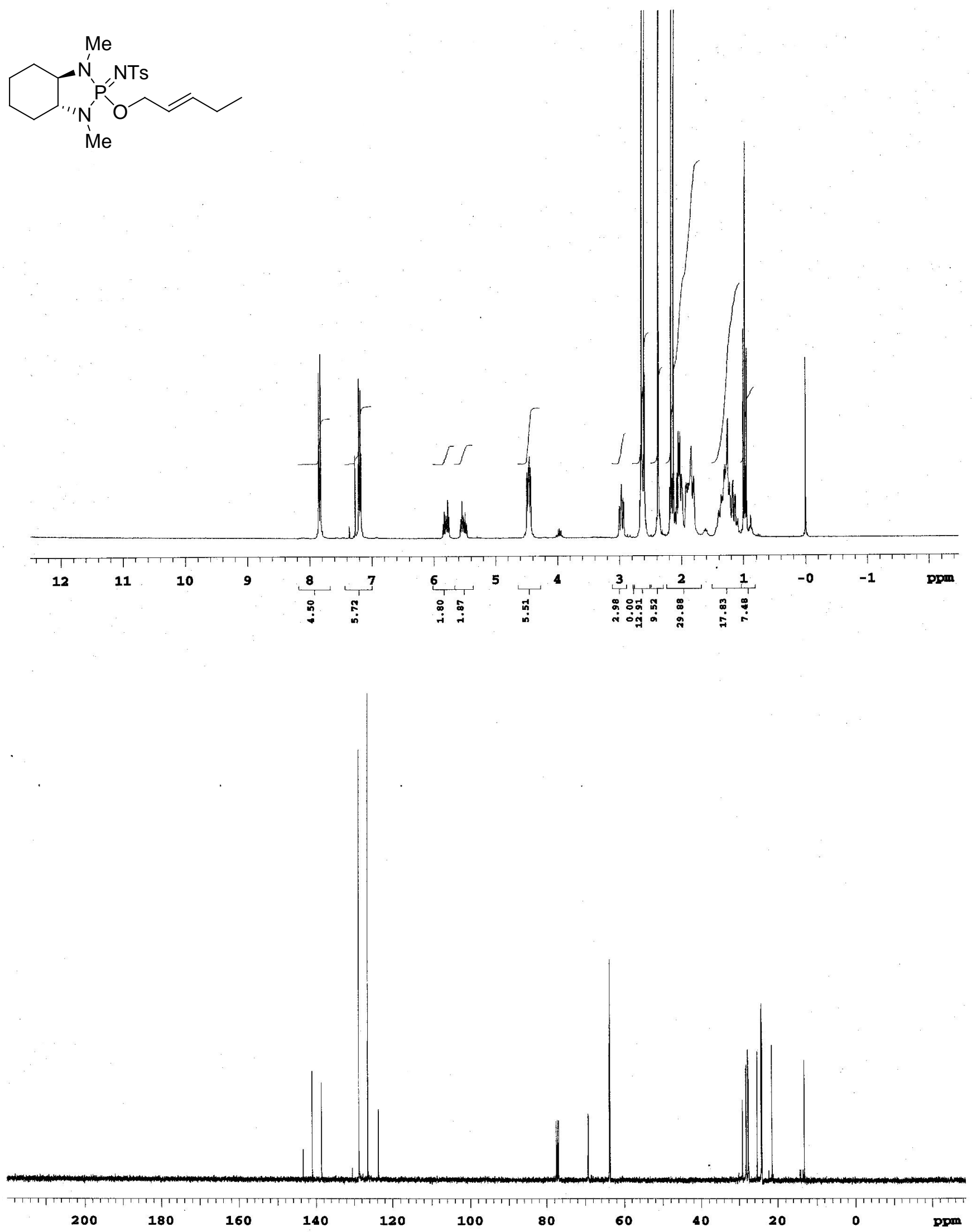

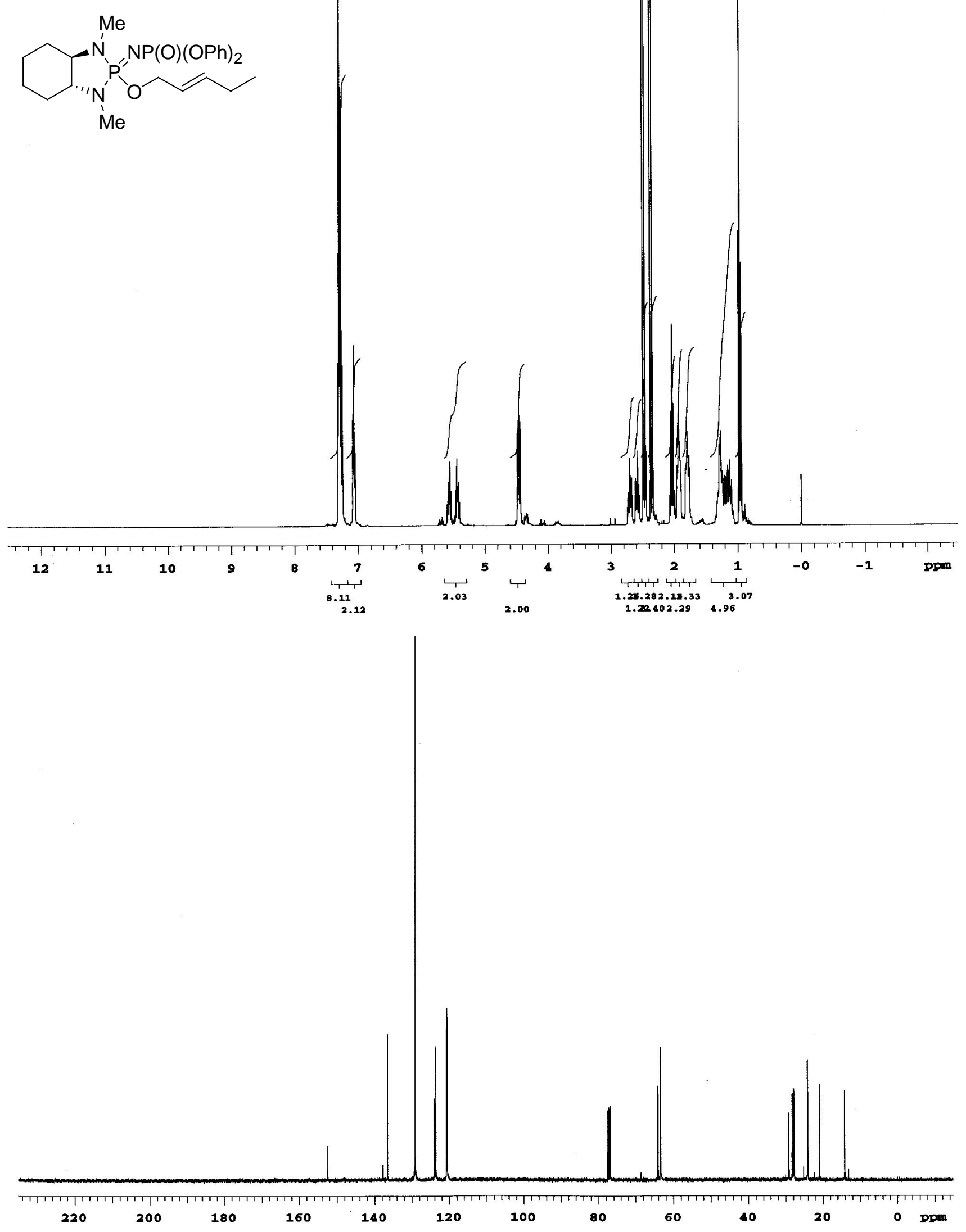
<smiles>CC/C=C\CO[PH]1(N(C)C)N(C)C2CCCCC2N1C</smiles>
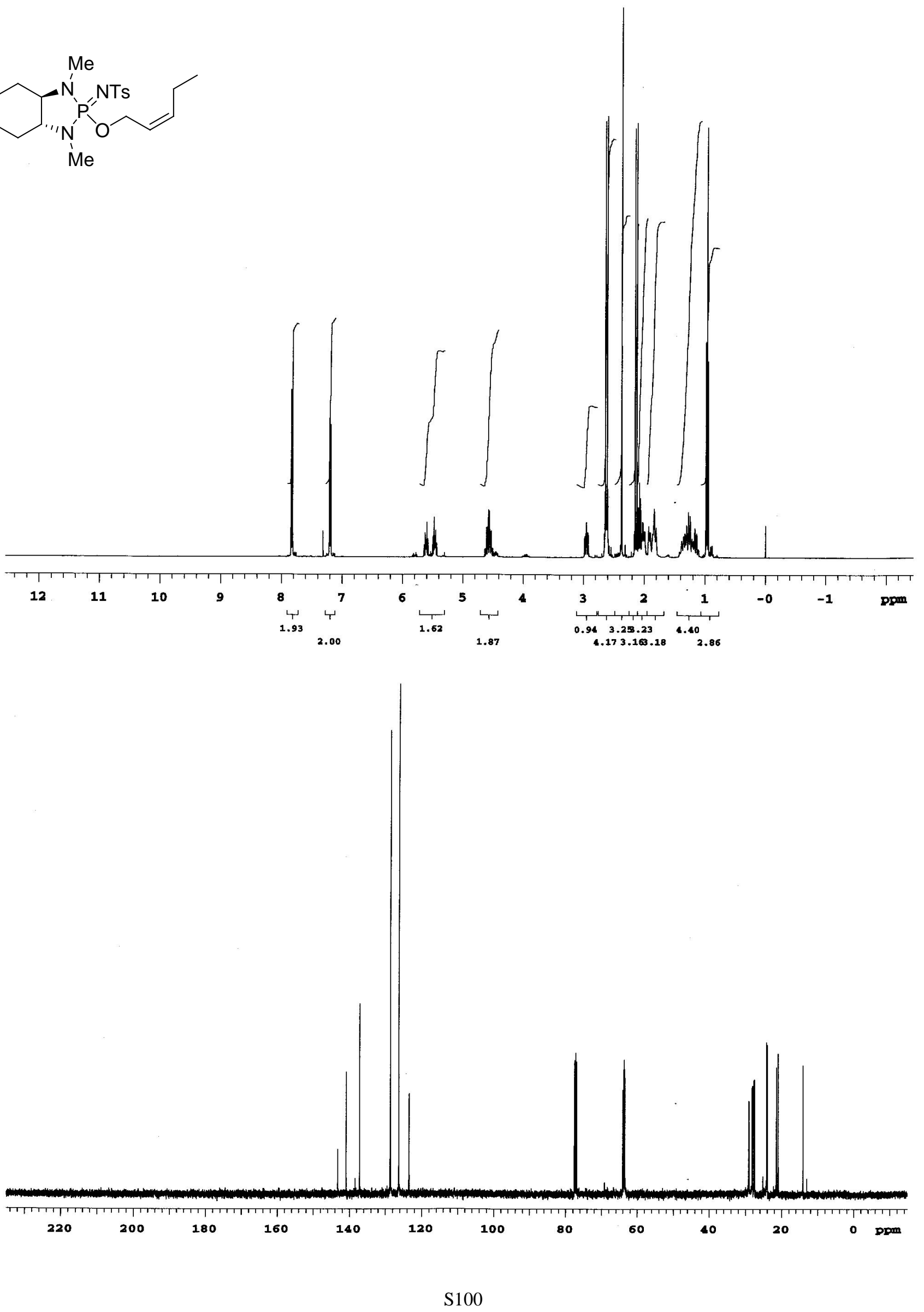

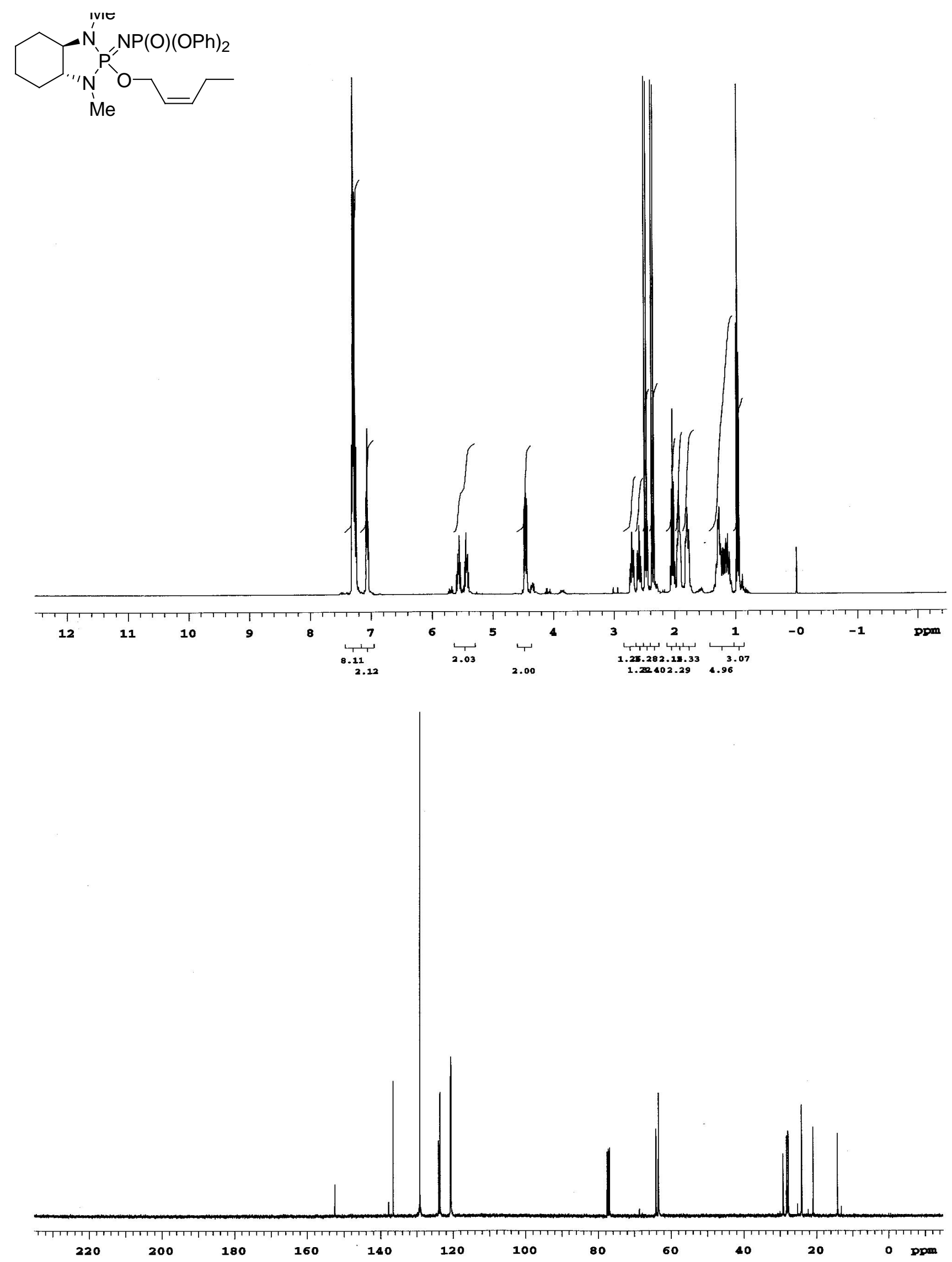
<smiles>CC/C=C/COP1(=[N-])N(C(C)C)C2CCCC[C@H]2N1C(C)C</smiles>
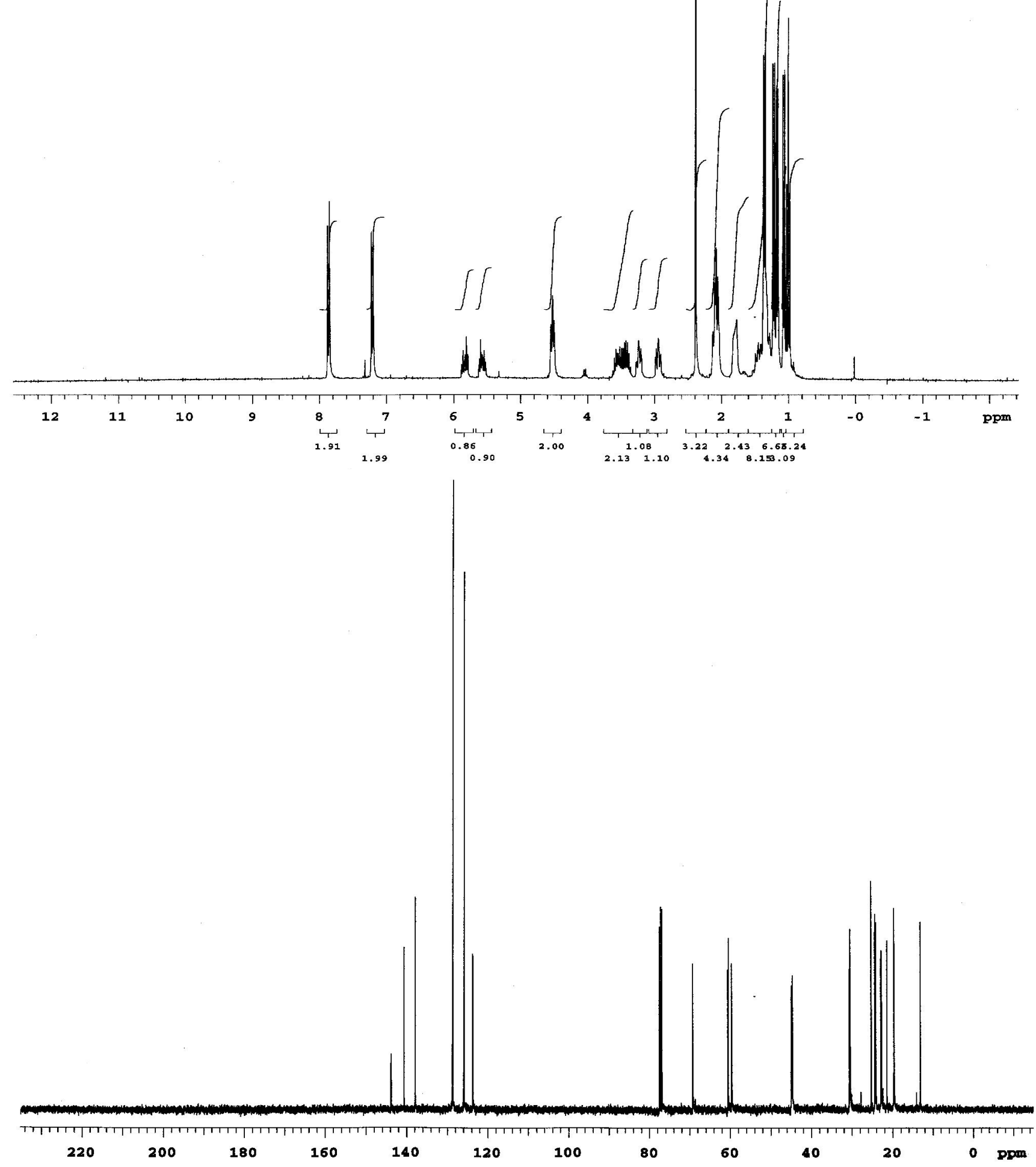

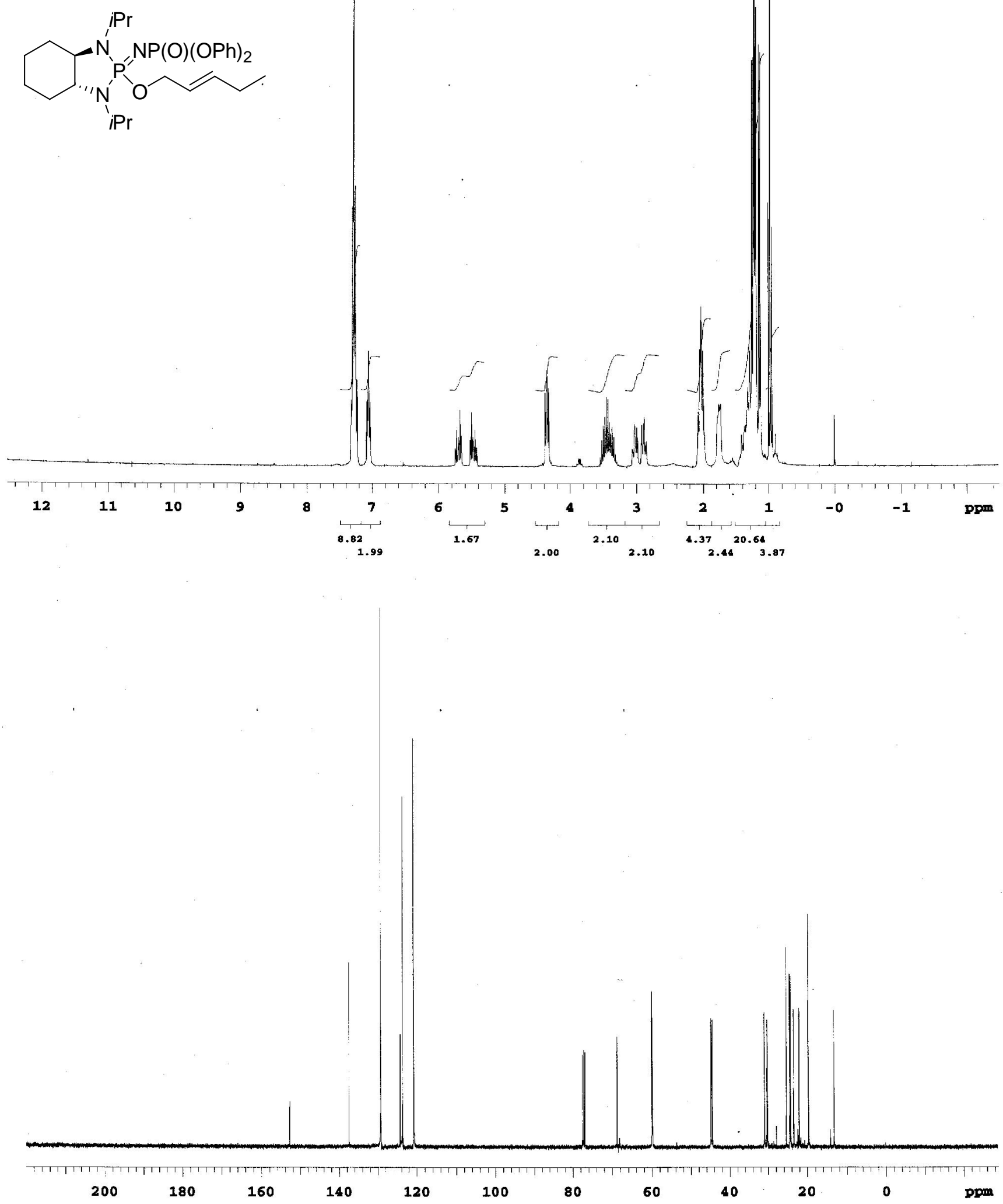

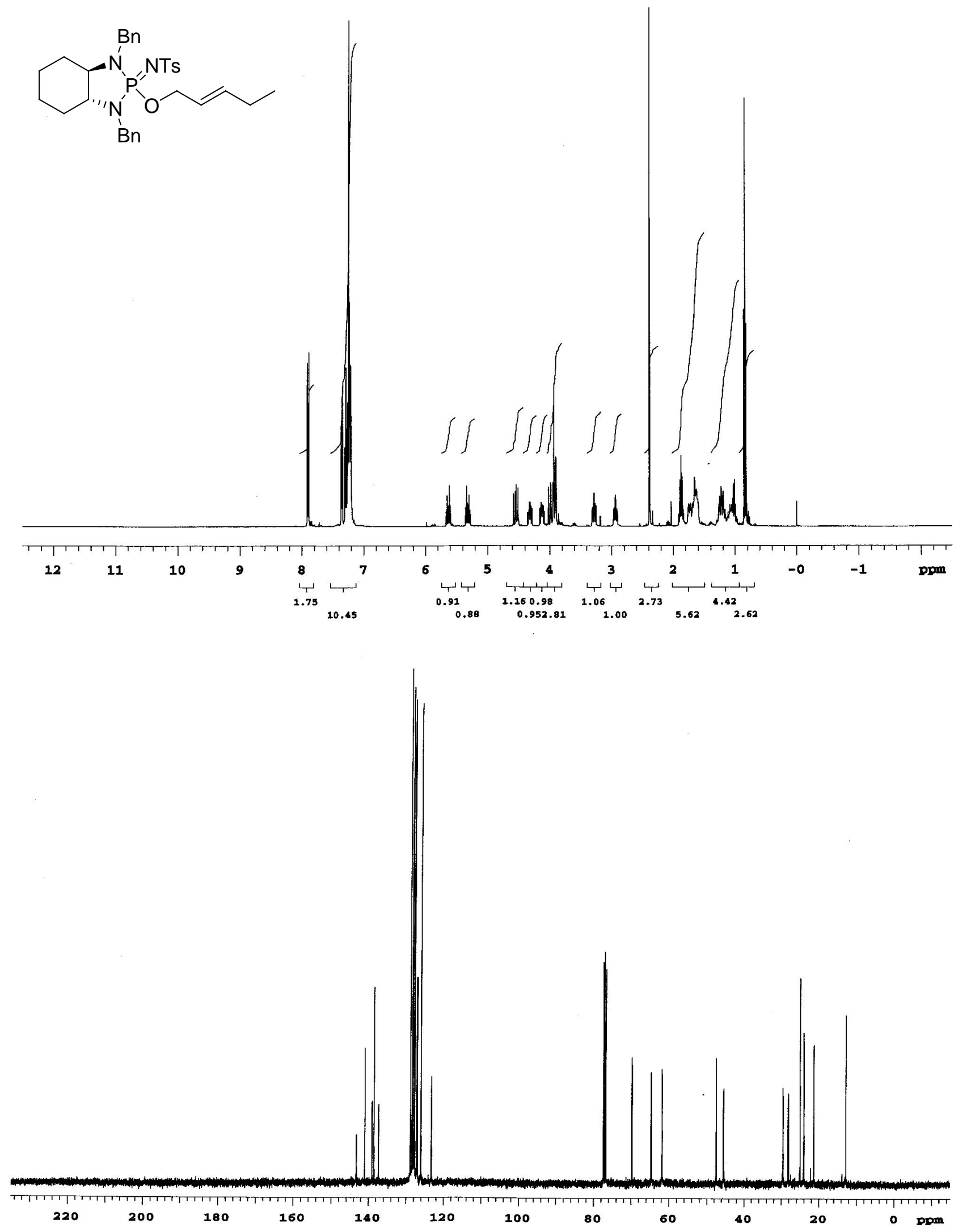

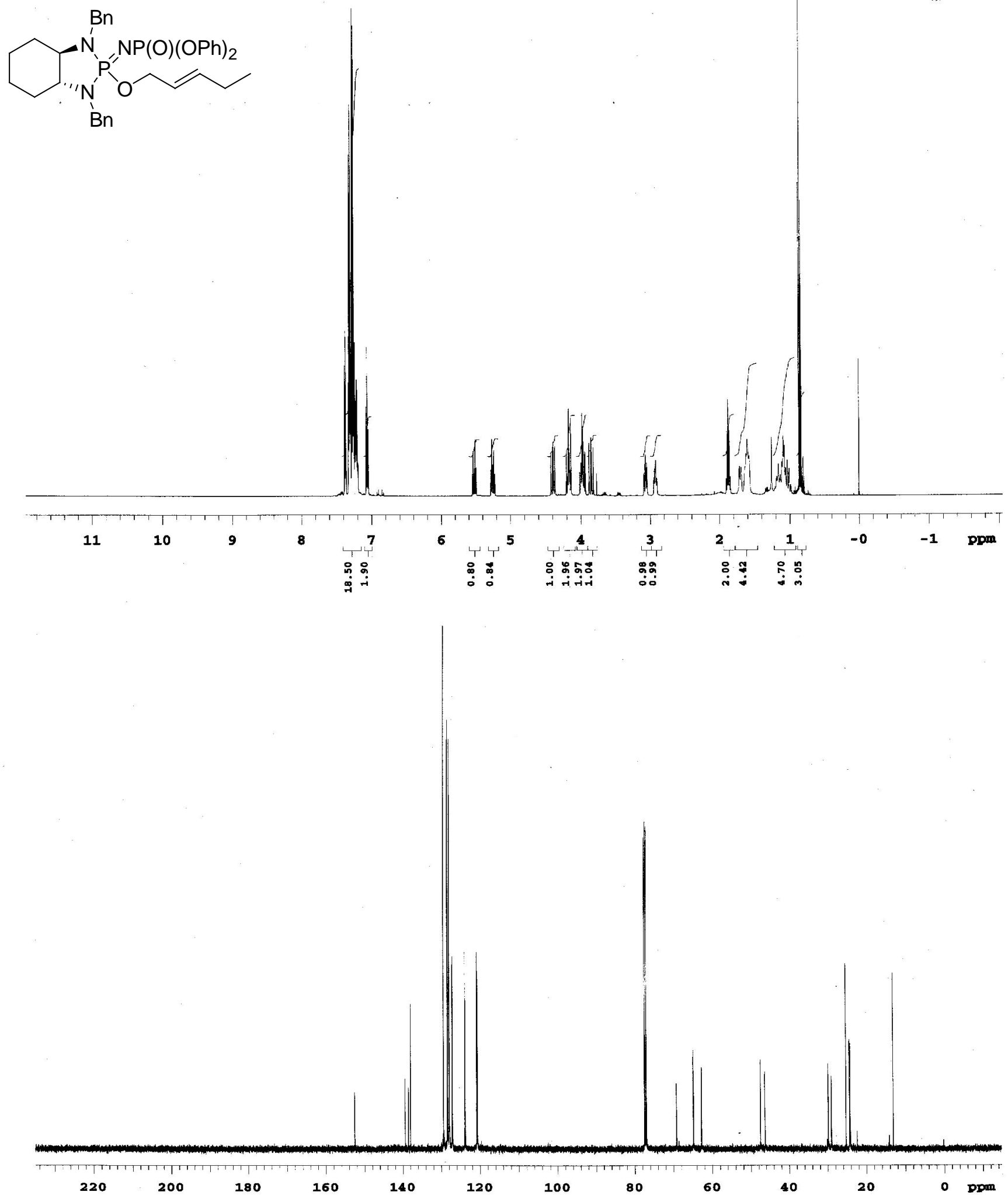
<smiles>C=CC(CC)N([13CH3])P1(=O)N(C)[C@H]2CCCC[C@H]2N1C</smiles>
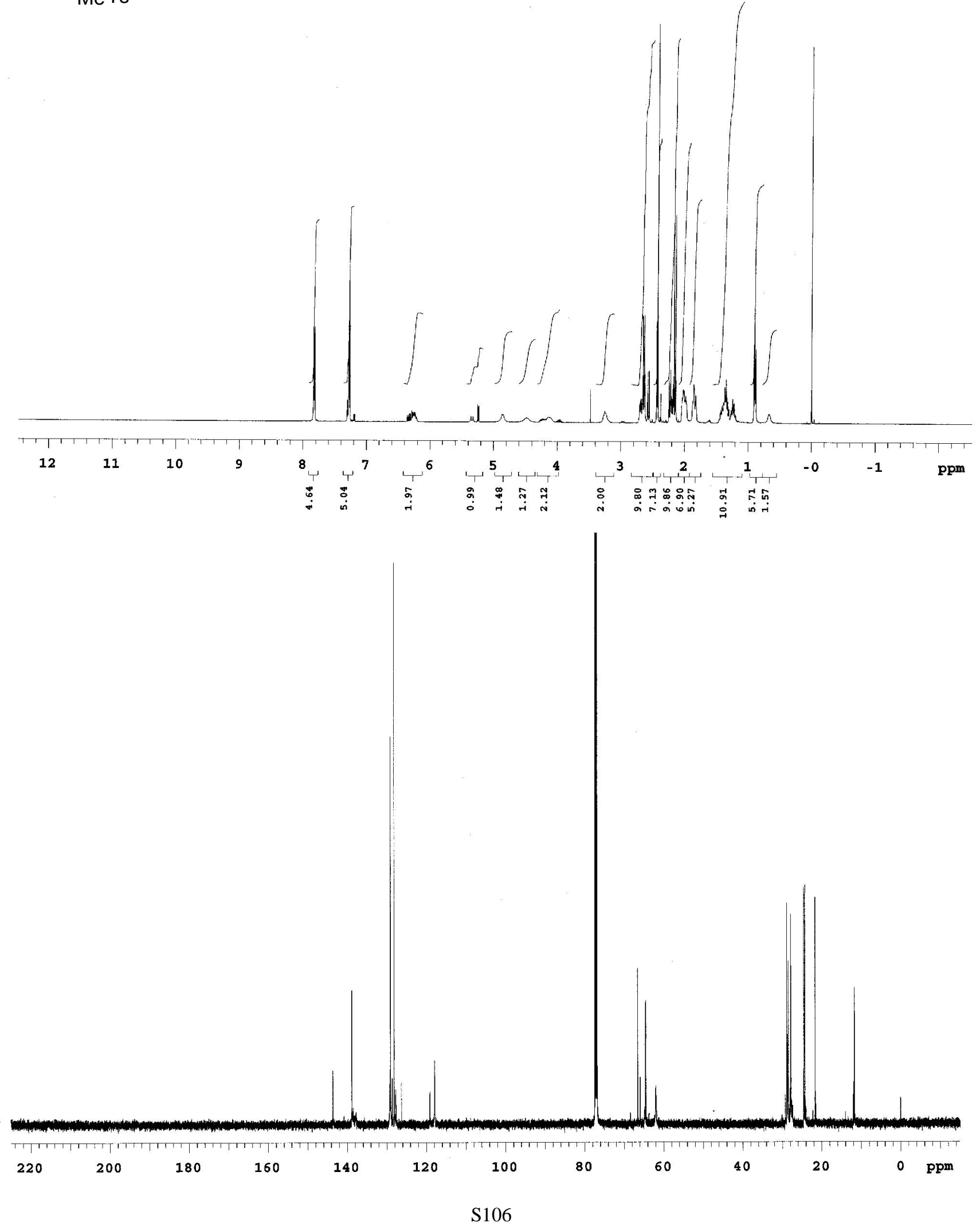

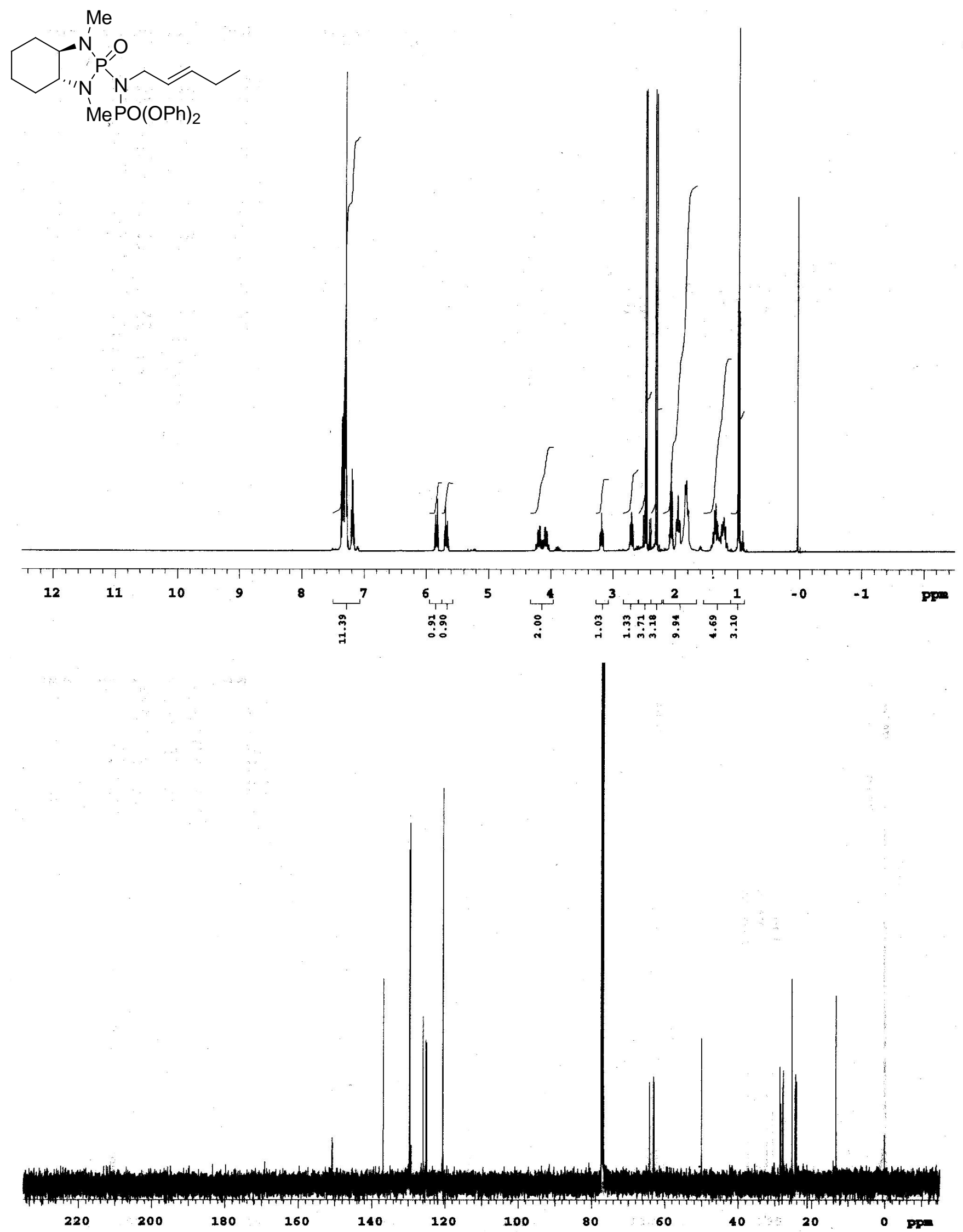


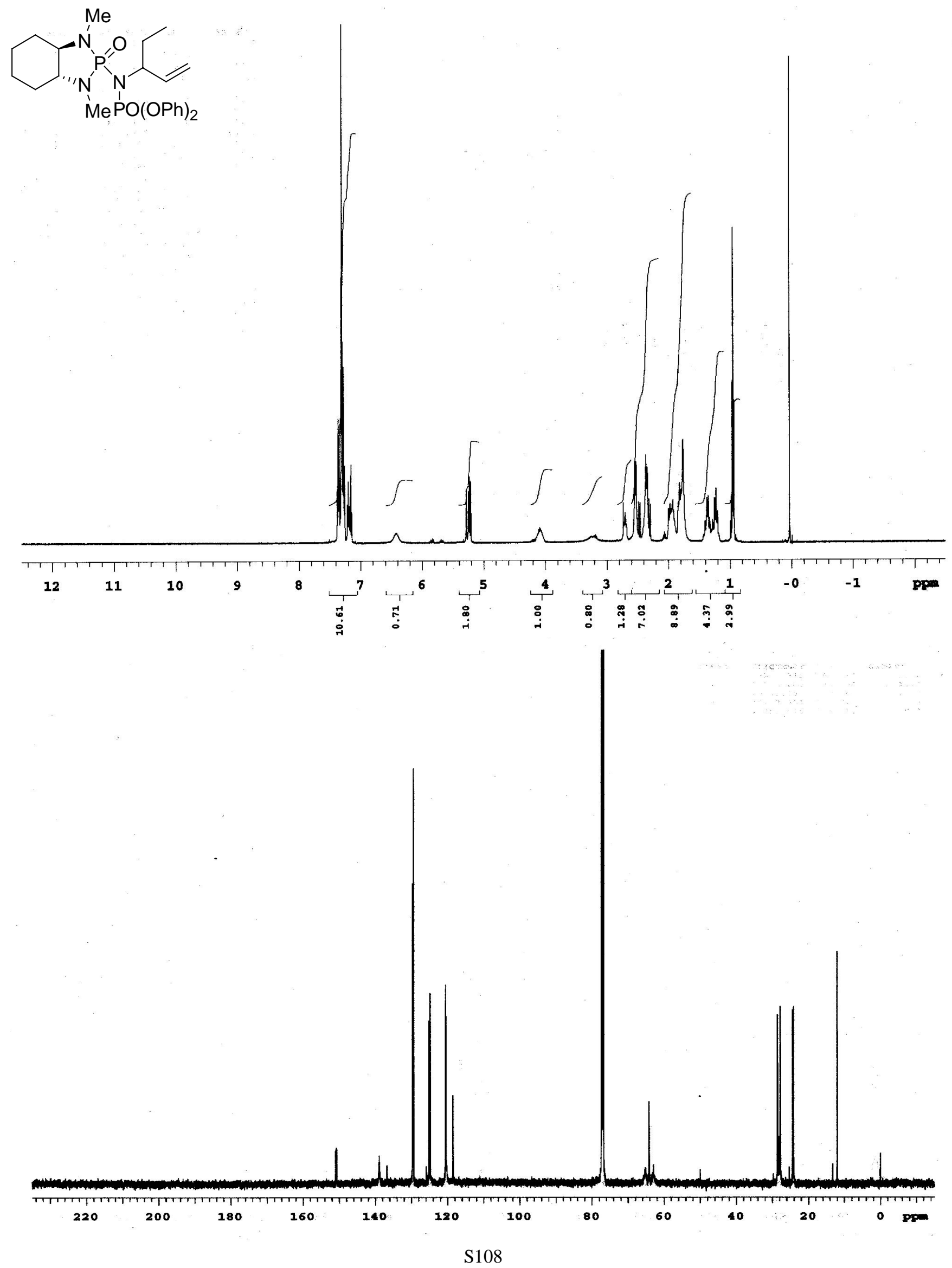


<smiles>C=CC(CC)N(C)P1(=O)N(C(C)C)[C@@H]2CCCC[C@H]2N1C(C)C</smiles>
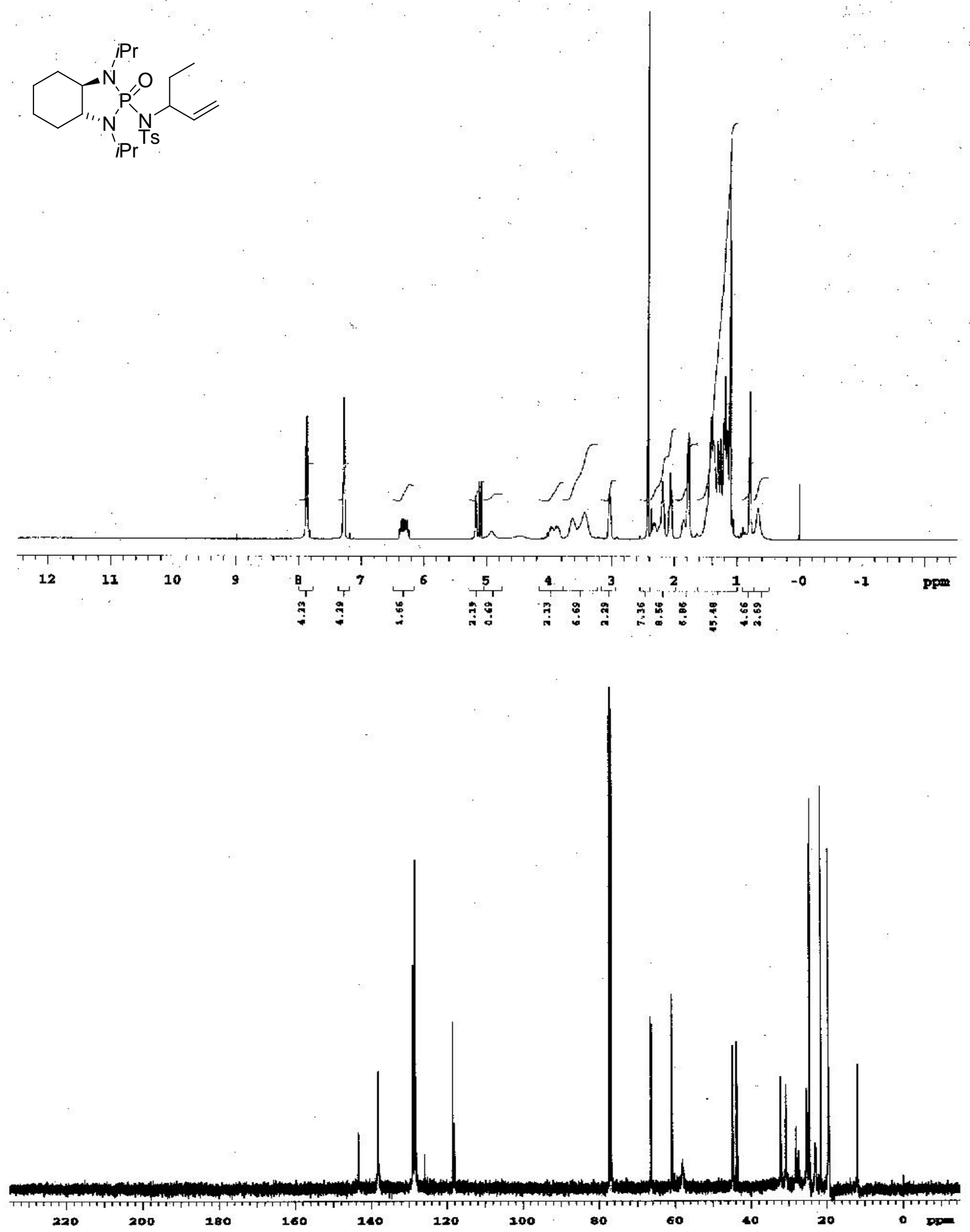

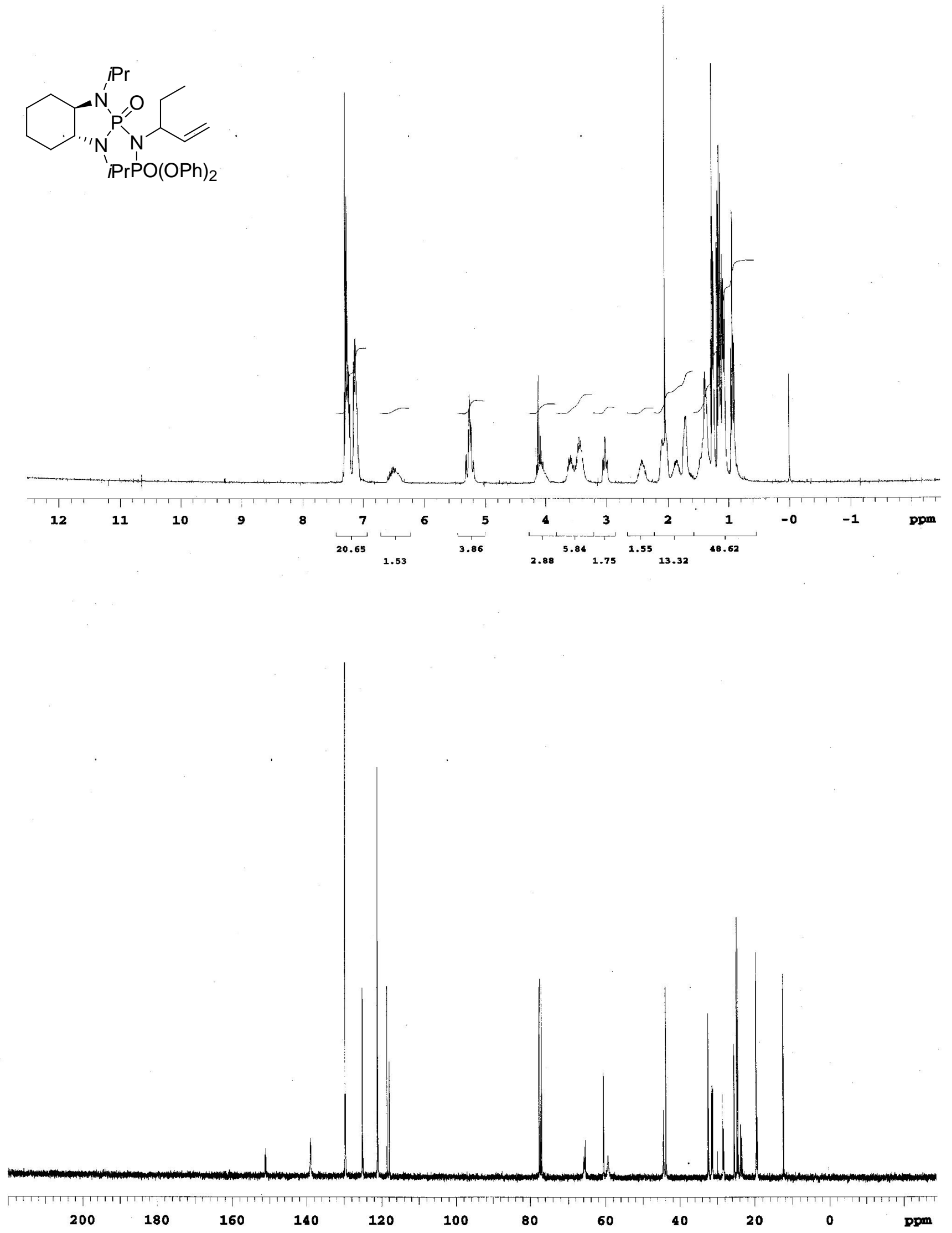

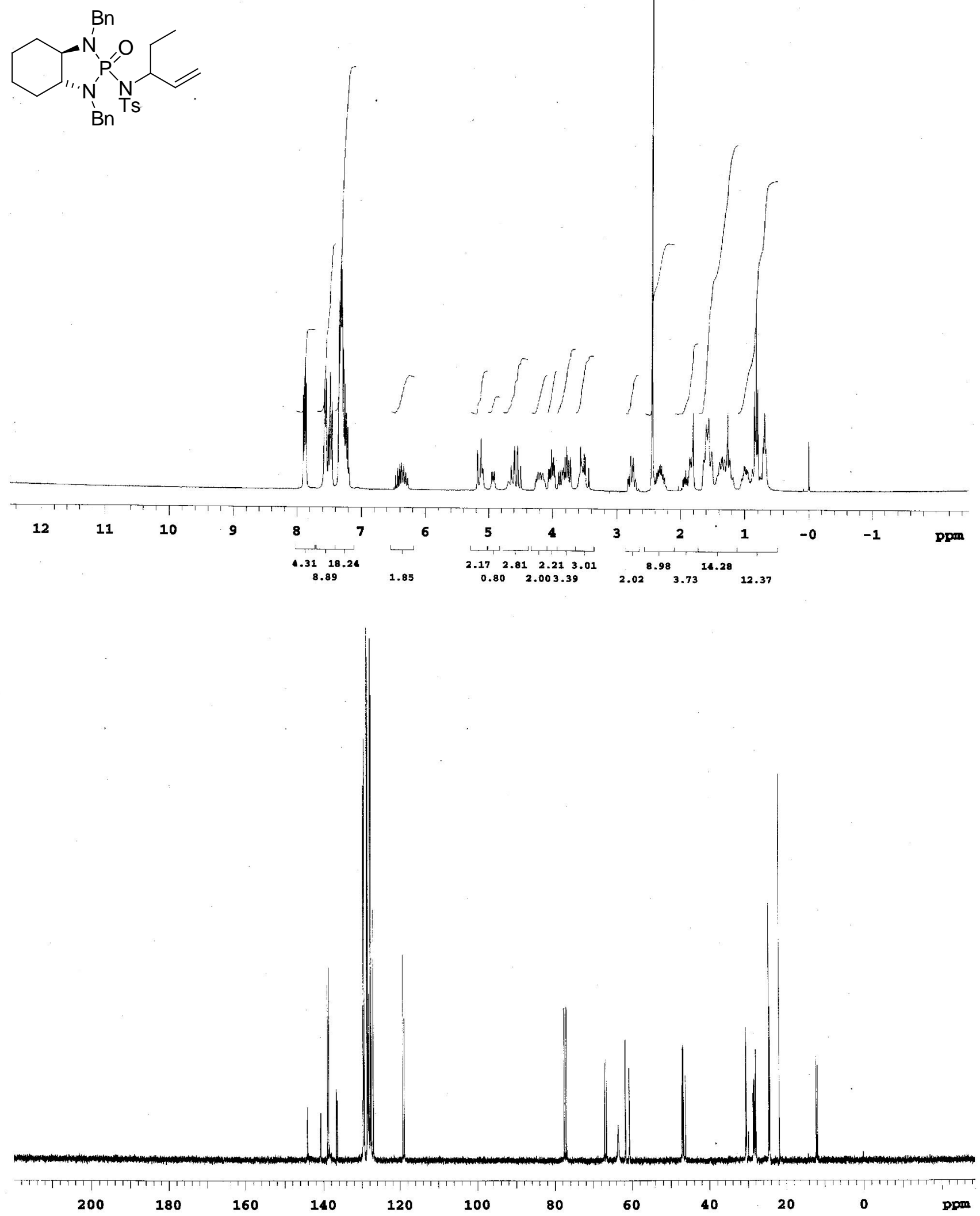

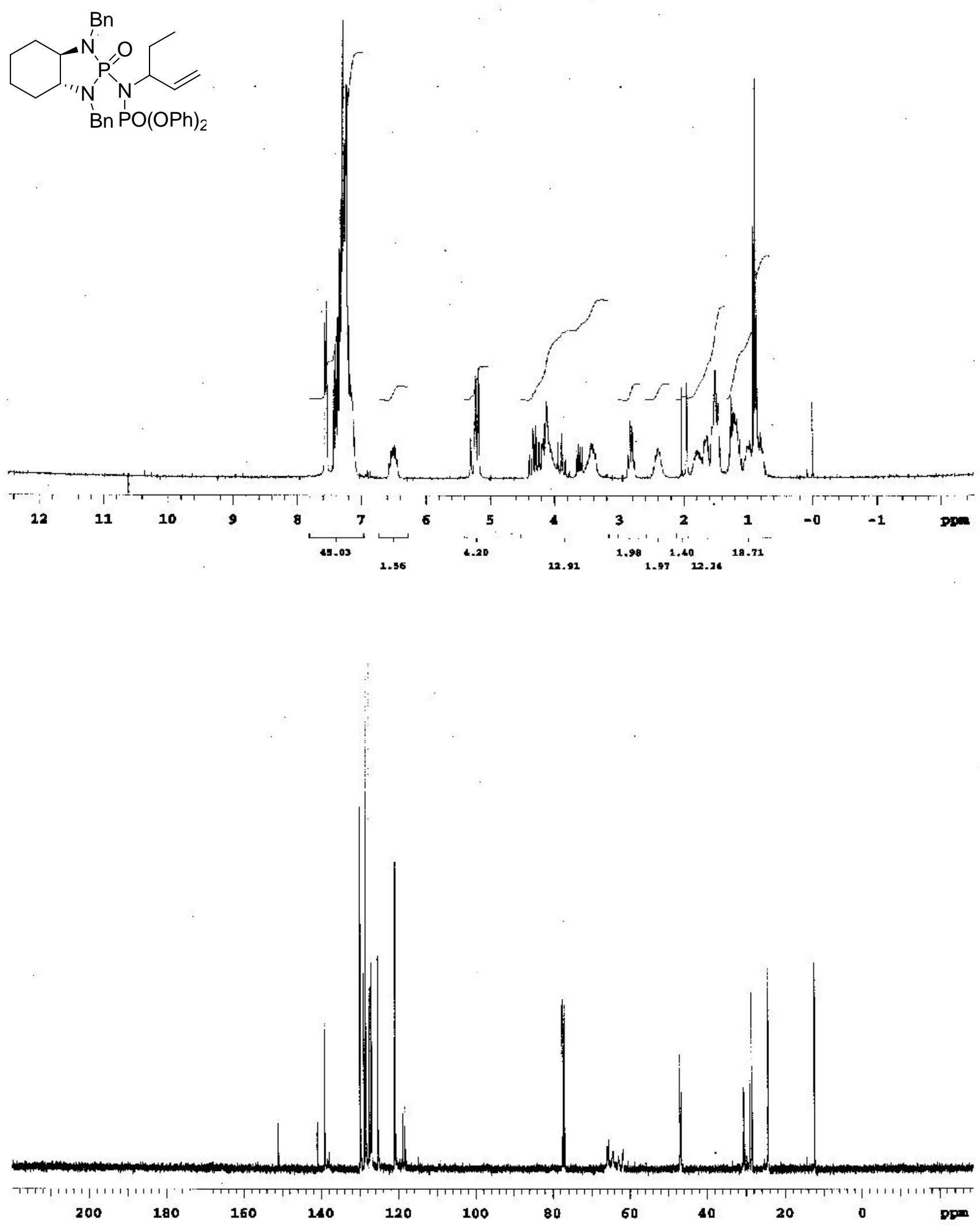